\title{
M2-brane surface operators and gauge theory dualities in Toda
}

\author{
Jaume Gomis ${ }^{a}$ and Bruno Le Floch ${ }^{a, b}$ \\ ${ }^{a}$ Perimeter Institute for Theoretical Physics, \\ Waterloo, Ontario, N2L 2Y5, Canada \\ ${ }^{b}$ Laboratoire de Physique Théorique de l'École Normale Supérieure, ${ }^{1}$ \\ Paris, 75005, France \\ E-mail: jgomis@perimeterinstitute.ca, blefloch@princeton.edu
}

AbSTRACT: We give a microscopic two dimensional $\mathcal{N}=(2,2)$ gauge theory description of arbitrary M2-branes ending on $N_{f}$ M5-branes wrapping a punctured Riemann surface. These realize surface operators in four dimensional $\mathcal{N}=2$ field theories. We show that the expectation value of these surface operators on the sphere is captured by a Toda CFT correlation function in the presence of an additional degenerate vertex operator labelled by a representation $\mathcal{R}$ of $\mathrm{SU}\left(N_{f}\right)$, which also labels M2-branes ending on M5-branes. We prove that symmetries of Toda CFT correlators provide a geometric realization of dualities between two dimensional gauge theories, including $\mathcal{N}=(2,2)$ analogues of Seiberg and Kutasov-Schwimmer dualities. As a bonus, we find new explicit conformal blocks, braiding matrices, and fusion rules in Toda CFT.

KEYwords: Supersymmetry and Duality, Conformal and W Symmetry, M-Theory, Extended Supersymmetry

ARXIV EPRINT: 1407.1852

\footnotetext{
${ }^{1}$ Unité mixte (UMR 8549) du CNRS et de l'ENS, Paris.
} 


\section{Contents}

1 Introduction and conclusions 1

2 Surface operators as Toda degenerate operators $\quad 10$

$\begin{array}{lll}2.1 & \text { SQED and Toda fundamental degenerate } & 17\end{array}$

$\begin{array}{ll}\text { 2.1.1 Expanding the SQED partition function } & 19\end{array}$

2.1.2 Matching parameters for SQED 22

2.2 SQCD and Toda antisymmetric degenerate 24

2.2.1 Expanding the SQCD partition function 26

$\begin{array}{ll}2.2 .2 & \text { Matching parameters for SQCD }\end{array}$

$\begin{array}{ll}2.2 .3 & \text { Decoupled multiplets and irregular puncture }\end{array}$

2.3 SQCDA and Toda symmetric degenerate 33

2.3.1 Expanding the SQCDA partition function 34

$\begin{array}{ll}\text { 2.3.2 Matching parameters for SQCDA } & 36\end{array}$

$\begin{array}{ll}\text { 2.3.3 Adding a superpotential to SQCDA } & 37\end{array}$

2.4 Quivers and multiple Toda degenerates 41

2.4.1 Matching parameters for quivers 44

2.4.2 Arbitrary Toda degenerates 48

3 Gauge theory dualities as Toda symmetries $\quad \mathbf{5 0}$

$\begin{array}{ll}3.1 \text { Seiberg duality as momentum conjugation } & 51\end{array}$

3.1.1 Momentum conjugation for $\widetilde{N_{f}}=N_{f} \quad 52$

3.1.2 Decoupling multiplets towards $\widetilde{N}_{f}<N_{f} \quad 54$

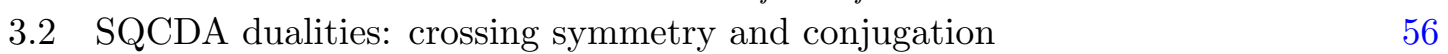

$\begin{array}{lll}3.2 .1 & (2,2)^{*} \text {-like duality as a braiding move } & 56\end{array}$

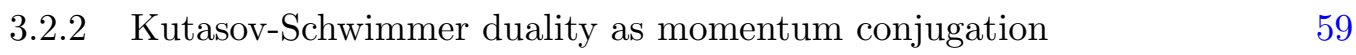

$\begin{array}{lll}3.3 & \text { Dualities for quivers } & 61\end{array}$

3.3.1 Seiberg dualities from braiding moves 63

3.3.2 Seiberg dualities from momentum conjugation 66

$\begin{array}{ll}\text { A Toda CFT } & \mathbf{7 0}\end{array}$

$\begin{array}{lll}\text { A.1 Basic properties } & 70\end{array}$

$\begin{array}{lll}\text { A.2 Three-point functions } & 73\end{array}$

$\begin{array}{lll}\text { A.3 Braiding matrices } & 76\end{array}$

$\begin{array}{lll}\text { A.3.1 Gauge theory transfer matrices } & 76\end{array}$

$\begin{array}{lll}\text { A.3.2 Toda CFT braiding matrices } & 78\end{array}$

A.4 Fusion rules $\quad 81$

A.4.1 Fusion of two semi-degenerate operators $\quad 82$

A.4.2 Fusion of semi-degenerate and degenerate operators $\quad 84$

$\begin{array}{lll}\text { A.5 Conformal blocks } & 86\end{array}$

$\begin{array}{lll}\text { A.6 } & \text { Irregular punctures } & 88\end{array}$ 
A.6.1 OPE with the stress-energy tensor $\quad 89$

$\begin{array}{lll}\text { A.6.2 Free field realization } & 91\end{array}$

A.6.3 Two-point functions 93

B Vortex partition function dualities $\quad 95$

B.1 SQCD vortex partition functions 95

B.1.1 SQCD with $\widetilde{N_{f}}<N_{f} \quad 96$

B.1.2 SQCD with $\widetilde{N_{f}}=N_{f} \quad 98$

B.2 SQCDA vortex partition functions 100

B.2.1 Preliminary result for $N_{f}=1 \quad 101$

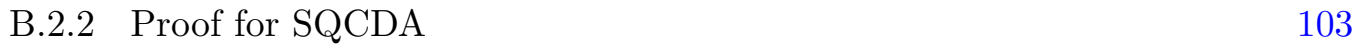

\section{Introduction and conclusions}

The traditional order parameters for the phases of four dimensional gauge theories are the Wilson [1] and 't Hooft [2] operators. In recent years, the construction of nonlocal surface operators [3], which insert probe strings, have enlarged the space of order parameters of gauge theories. Indeed, surface operators can distinguish phases which are otherwise indistinguishable using the Wilson-'t Hooft criteria [4].

A surface operator can be defined either by specifying a codimension-two singularity for the elementary fields or by coupling a two dimensional field theory to the bulk four dimensional one [3]. The couplings between bulk and defect degrees of freedom can result in rich dynamics for the combined system, arising from the synergy of two dimensional and four dimensional strong coupling dynamics. For a sample of early references on surface operators see [5-12].

Surface operators also play a fundamental role in the six dimensional $\mathcal{N}=(2,0)$ supersymmetric field theory living on the worldvolume of a collection of $N_{f}$ coincident and flat M5-branes. A class of surface operators in this theory are labeled by a representation $\mathcal{R}$ of $A_{N_{f}-1}$ and admit an M-theory realization as a collection of M2-branes ending on the M5-branes along the domain of support of the surface operator.

In this paper we give a microscopic two dimensional gauge theory description of all such surface operators when the M5-branes wrap a punctured Riemann surface $C$ [13]. This realizes a surface operator in a four dimensional $\mathcal{N}=2$ gauge theory.

\begin{tabular}{llllllll} 
& & & \multicolumn{5}{c}{${ }^{C}$} \\
M5 & 0 & 1 & 2 & 3 & 4 & 5 & \\
M2 & 0 & 1 & & & & & 6 \\
\hline
\end{tabular}

The surface operator associated to a collection of M2-branes labeled by a representation $\mathcal{R}$ of $A_{N_{f}-1}$ corresponds to the following two dimensional $\mathcal{N}=(2,2)$ gauge theory

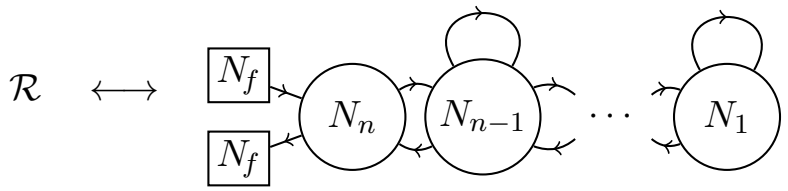


coupled to the bulk theory. A cubic superpotential couples each adjoint chiral multiplet to the neighboring bifundamental chiral multiplets. The FI parameters associated to $\mathrm{U}\left(N_{j}\right)$ for $j<n$ vanish. The ranks $N_{j}$ encode the representation $\mathcal{R}$ whose Young diagram

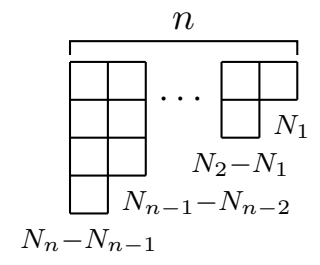

has $n$ columns with $N_{n}-N_{n-1} \geq N_{n-1}-N_{n-2} \geq \cdots \geq N_{2}-N_{1} \geq N_{1} \geq 0$ boxes. $^{1}$

Advances in the computation of supersymmetric partition functions of four dimensional $\mathcal{N}=2$ gauge theories on the squashed four-sphere $S_{b}^{4}[14,15]$ have resulted in exact formulas for the expectation value of Wilson [14] and 't Hooft operators [16] as functions of the gauge couplings and masses of the hypermultiplets. The gauge theory computation of the expectation value of surface operators supported on a squashed $S^{2} \subset S_{b}^{4}$ are not yet available. However, recent results in the exact computation of the two-sphere partition function of $\mathcal{N}=(2,2)$ supersymmetric field theories [17-20], when suitably coupled to those in $[14,15]$, provide a concrete avenue of investigation of the expectation value of half-BPS surface operators in four dimensional $\mathcal{N}=2$ theories on $S_{b}^{4}$ using Feynman path integrals.

For the four dimensional $\mathcal{N}=2$ theories obtained by wrapping M5-branes on punctured Riemann surfaces, also known as class $\mathrm{S}$ theories [13], the $S_{b}^{4}$ partition function in $[14,15]$ admits an elegant representation [21] (see also [22]) in terms of two dimensional Toda CFT correlation functions. In the correspondence between four dimensional $\mathcal{N}=2$ theories and Toda CFT, the expectation value of Wilson and 't Hooft operators on $S_{b}^{4}$ are realized as Toda CFT correlators in the presence of loops operators and topological webs [23-25] (see also [26-28]). Degenerate vertex operators in $A_{N_{f}-1}$ Toda CFT are conjectured to realize the insertion of a supersymmetric surface operator [23] (see also [18, 29-32]).

In this paper we identify the two dimensional $\mathcal{N}=(2,2)$ gauge theory that realizes an arbitrary degenerate operator in Toda CFT, which in turn corresponds to an arbitrary M2-brane configuration ending on wrapped M5-branes. ${ }^{2}$ A degenerate operator with Toda momentum $\alpha=-b \Omega$, where $\Omega$ is the highest weight vector of a representation $\mathcal{R}(\Omega)$ of $A_{N_{f}-1}$, corresponds to the quiver gauge theory (1.1). The complexified FI/theta parameter associated to the $\mathrm{U}\left(N_{n}\right)$ gauge group encodes the position of the degenerate puncture

\footnotetext{
${ }^{1}$ The highest weight of $\mathcal{R}$ is $\Omega=\sum_{j=1}^{n} \omega_{N_{j}-N_{j-1}}$ in terms of the fundamental weights $\omega_{K}$ of $A_{N_{f}-1}$.

${ }^{2}$ Another class of surface operators can be realized by M5-branes, and are labeled by a partition $\rho$ of $N_{f}$. It was conjectured in [33] that the instanton partition function of four dimensional $\mathcal{N}=2 \mathrm{SU}\left(N_{f}\right) \mathrm{SYM}$ in the presence of such an M5-defect labeled by $\rho$ is the norm of a Whittaker vector in the $W$-algebra $W_{\rho}$. Some checks of this conjecture and generalizations have appeared in [34-41]. We propose that the surface operator associated to an M5-defect labeled by $\rho$, with $N_{f}=K_{1}+\cdots+K_{n}$, corresponds to coupling the bulk $\mathcal{N}=2$ superconformal field theory to the two dimensional $\mathcal{N}=(2,2)$ gauge theory with $N_{j}=K_{1}+\cdots+K_{j}$ : vacua of this theory yield a monodromy for the $4 \mathrm{~d}$ gauge field, which breaks $\mathrm{SU}\left(N_{f}\right)$ to $S\left[\mathrm{U}\left(K_{1}\right) \times \cdots \times \mathrm{U}\left(K_{n}\right)\right]$. Surface operators labeled by a Young diagram have appeared in [42].
} 
(the other FI parameters must vanish in this correspondence). The surface operator is supported on an $S^{2} \subset S_{b}^{4}$ invariant under the $\mathrm{U}(1) \times \mathrm{U}(1)$ isometries of $S_{b}^{4}{ }^{3}$

The quiver gauge theory (1.1) can be used to construct a surface operator in any four dimensional $\mathcal{N}=2$ gauge theory that contains an $\mathrm{SU}\left(N_{f}\right) \times \mathrm{SU}\left(N_{f}\right) \times \mathrm{U}(1)$ flavour or gauge symmetry group. This is the flavour symmetry of the chiral multiplets charged only under the $\mathrm{U}\left(N_{n}\right)$ gauge group factor in (1.1). A surface operator is constructed by identifying the common $\mathrm{SU}\left(N_{f}\right) \times \mathrm{SU}\left(N_{f}\right) \times \mathrm{U}(1)$ symmetry groups of the four dimensional and two dimensional theories.

The simplest four dimensional $\mathcal{N}=2$ class $\mathrm{S}$ theory in which we can include a surface defect is the theory of $N_{f}^{2}$ hypermultiplets. This theory is realized by wrapping $N_{f}$ M5branes on a trinion with two full and one simple puncture. We explicitly show that the partition function of this theory in the presence of the surface operator labeled by a representation $\mathcal{R}(\Omega)$ is given by the Toda four-point function ${ }^{4}$ obtained by adding to the trinion a degenerate field with momentum $\alpha=-b \Omega$

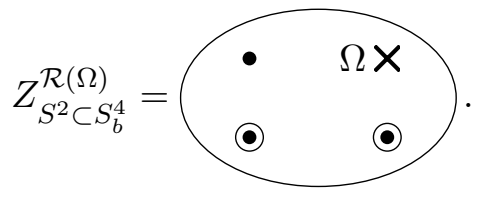

The two dimensional quiver gauge theory (1.1) is coupled to the four dimensional field theory by (weakly) gauging the $\mathrm{SU}\left(N_{f}\right) \times \mathrm{SU}\left(N_{f}\right) \times \mathrm{U}(1)$ flavour symmetry associated to the trinion. The coupling can also be described by a cubic superpotential between the bulk hypermultiplets and the fundamental and antifundamental chiral multiplets on the defect. The combined $4 \mathrm{~d} / 2 \mathrm{~d}$ quiver diagram describing the insertion of the surface operator in this four dimensional theory is

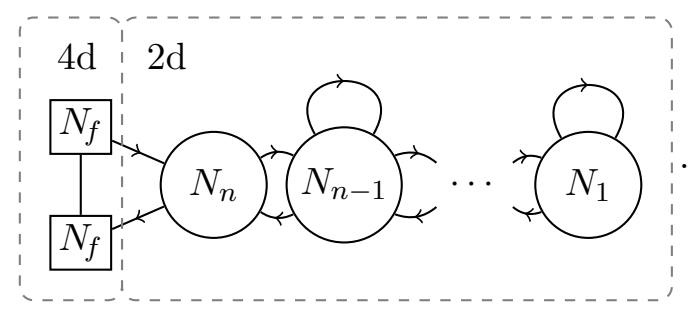

This construction can be enriched by allowing one (or both) of the $\mathrm{SU}\left(N_{f}\right)$ flavour symmetry groups of (1.1) to be coupled to one (or two) $\mathrm{SU}\left(N_{f}\right)$ gauge group factors of a four dimensional theory. An interesting theory where such surface operators can be inserted is four dimensional $\mathcal{N}=2$ superconformal SQCD. The SQCD quiver description

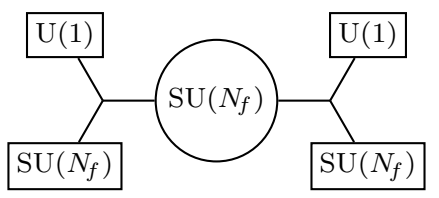

\footnotetext{
${ }^{3}$ Degenerate operators with momentum $\alpha=-\Omega / b$ correspond to the same quiver gauge theory but now supported on the other $\mathrm{U}(1) \times \mathrm{U}(1)$ invariant $S^{2} \subset S_{b}^{4}$. The most general degenerate momentum $\alpha=-b \Omega-\Omega^{\prime} / b$ corresponds to the insertion of the associated surface operators on both $S^{2}$ 's, but with a non-trivial coupling at their intersection points, namely the poles of $S_{b}^{4}$.

${ }^{4}$ The four point function in (1.3) contains full $\bullet$, simple $\bullet$, and degenerate $\mathbf{X}$ punctures.
} 
makes an $\mathrm{U}\left(N_{f}\right)^{2}$ flavour symmetry manifest. Both sides of the quiver represent a hypermultiplet transforming in the bifundamental representation of the $\mathrm{SU}\left(N_{f}\right)$ gauge group and a $\mathrm{U}\left(N_{f}\right)$ flavour group. The two dimensional gauge theory (1.1) can now be coupled to SQCD by identifying the two dimensional flavour symmetry with the $\mathrm{U}\left(N_{f}\right)$ flavour symmetry of either of these hypermultiplets and the $\mathrm{SU}\left(N_{f}\right)$ gauge group. The two resulting surface operators in SQCD are realized by the following 4d/2d quiver diagrams (we introduce the hybrid node $\square$ to denote a four dimensional gauge group which gauges a two dimensional flavour symmetry):
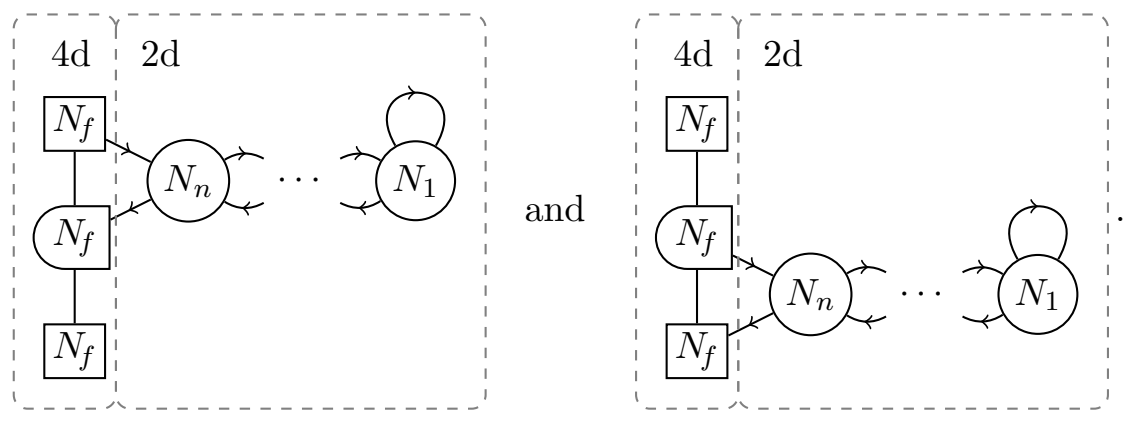

The correspondence we propose between these surface operators and Toda CFT correlators predicts a duality between the two coupled $4 \mathrm{~d} / 2 \mathrm{~d}$ theories in (1.6), since $\mathrm{SU}\left(N_{f}\right)$ SQCD is the theory on $N_{f}$ M5-branes wrapping a sphere with two full and two simple punctures. The weakly coupled regime of SQCD corresponds to a pants decomposition where the two simple punctures belong to distinct trinions, which are joined by a thin tube. In this framework, coupling the two dimensional theory (1.1) to either of the two hypermultiplets in SQCD correspond to inserting a degenerate operator with momentum $\alpha=-b \Omega$ in either trinion. The partition functions of the two surface operators in SQCD are thus both realized as the same five-point function of two full, two simple, and an additional degenerate puncture:

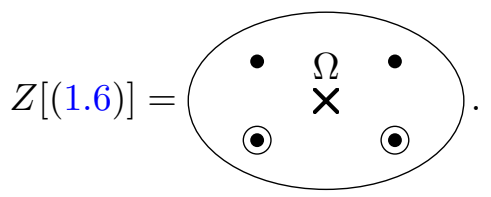

In this language, the two $4 \mathrm{~d} / 2 \mathrm{~d}$ quiver diagrams (1.6) correspond to two different degeneration limits of the five-point function. It is important to note that this "node-hopping" duality of the $4 \mathrm{~d} / 2 \mathrm{~d}$ theory is distinct from the usual S-duality of four dimensional $\mathcal{N}=2$ SQCD. The node-hopping duality was first observed in the superconformal index of some $4 \mathrm{~d} / 2 \mathrm{~d}$ theories in [42], whose $4 \mathrm{~d} / 2 \mathrm{~d}$ quiver notation we have borrowed. The superconformal index with surface operators has been considered in [43-46].

More generally, the surface operator (1.1) can be inserted in an arbitrary class S theory whenever the corresponding Riemann surface has at least one simple puncture. ${ }^{5}$

\footnotetext{
${ }^{5}$ Inserting multiple degenerate punctures near distinct simple punctures corresponds to including multiple surface operators built using distinct $\mathrm{SU}\left(N_{f}\right) \times \mathrm{SU}\left(N_{f}\right) \times \mathrm{U}(1)$ groups of the four dimensional theory. In a pants decomposition where the degenerate punctures are all inserted near the same simple puncture, the surface operator describes a single two dimensional gauge theory coupled through a given $\mathrm{SU}\left(N_{f}\right) \times \mathrm{SU}\left(N_{f}\right) \times \mathrm{U}(1)$ symmetry group.
} 
The generalized S-duality symmetry groupoid of a class S theory, which is realized as the Moore-Seiberg groupoid of the punctured Riemann surface, is enriched in the presence of surface operators. The addition of a degenerate puncture to the Riemann surface allows for further pants decomposition of the enriched Riemann surface, and thereby more duality transformations, that go beyond the dualities of the purely four dimensional theory. The node-hopping duality (1.6) provides an example of a new duality of the $4 \mathrm{~d} / 2 \mathrm{~d}$ system.

In the second part of the paper we "geometrize" dualities of two dimensional $\mathcal{N}=(2,2)$ quiver gauge theories in terms of symmetries of Toda CFT correlation functions. The quiver gauge theories we consider are

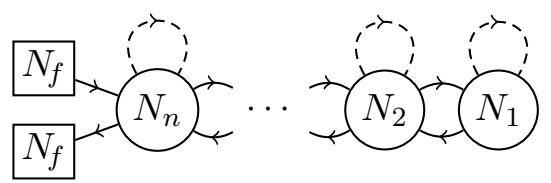

where an adjoint chiral multiplet can be added to any gauge group factor. Each adjoint chiral multiplet is coupled to the neighboring bifundamental chiral multiplets through a cubic superpotential, while nodes without an adjoint chiral multiplet have a quartic superpotential for the neighboring bifundamental chiral multiplets. Finally, the $N_{f}$ fundamental and antifundamental chiral multiplets have no superpotential coupling.

We show that surface operators obtained by coupling these two dimensional gauge theories (1.8) to class $\mathrm{S}$ theories have a Toda CFT realization. The quiver with $n$ gauge nodes corresponds to the insertion of $n$ degenerate fields labeled by either symmetric or antisymmetric representations of $A_{N_{f}-1}$. The $n$ complexified FI parameters encode the position of the $n$ degenerate punctures. We now build the representations labeling degenerate punctures recursively from the matter content of (1.8). If the $\mathrm{U}\left(N_{n}\right)$ factor has an adjoint chiral multiplet, then the representation carried by the $n$-th puncture is of symmetric type, and otherwise of antisymmetric type. Then sequentially for each gauge group factor $\mathrm{U}\left(N_{j}\right)$ from $j=n-1$ to 1 , the $j$-th puncture is labeled by a representation of the same type as the $(j+1)$-th puncture if there is an adjoint chiral multiplet, and otherwise by a representation of the other type. The Young diagram labeling the $j$-th puncture has $N_{j}-N_{j-1}$ boxes for $1 \leq j \leq n$, where $N_{0}=0$. See table 1 for useful special cases and figure 1 for a concrete example. The sphere partition function of the surface operator inserted by (1.8) in the trinion theory of free hypermultiplets is the Toda CFT correlator

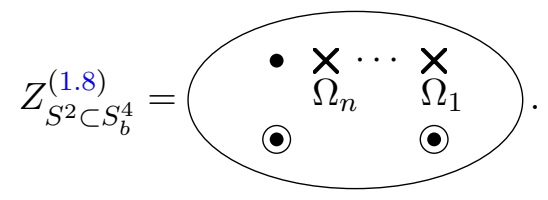

We also identify the gauge theory corresponding to multiple degenerate punctures labeled by arbitrary representations of $A_{N_{f}-1}$.

We consider several dualities in two dimensional $\mathcal{N}=(2,2)$ theories, realized as symmetries of the corresponding Toda CFT degenerate operators. As described below (see also table 2), some dualities correspond to the crossing symmetry exchanging two degenerate operators, while others correspond to conjugating all Toda CFT momenta, under which 


\begin{tabular}{|llll|}
\hline 2d Gauge theory & Field content & Representation & Equation \\
\hline SQED & Sundamental & (2.19) p. 17 \\
SQCD & Antisymmetric & (2.51) p. 24 \\
with $W=\sum_{t} \widetilde{q}_{t} X^{l_{t}} q_{t}$ \\
with $W=\operatorname{Tr} X^{l+1}$
\end{tabular}

Table 1. Correspondence between surface operators defined by $\mathcal{N}=(2,2)$ gauge theories and degenerate vertex operators labeled by representations of $A_{N_{f}-1}$. The positions of degenerate operators are controlled by a combination $\hat{z}$ of FI and theta parameters for each gauge group $\mathrm{U}(N)$, which differs from $z=e^{-2 \pi \xi+\mathrm{i} \vartheta}$ by a sign: $(-1)^{n_{f}}$ if the group has an adjoint chiral multiplet and otherwise $(-1)^{n_{f}+N-1}$, where $n_{f}$ is the number of fundamental chiral multiplets for that group.

The $\mathrm{U}\left(N_{1}\right) \times \cdots \times \mathrm{U}\left(N_{4}\right)$ linear quiver given below has adjoint chiral multiplets for
$\mathrm{U}\left(N_{1}\right)$ and $\mathrm{U}\left(N_{4}\right)$, hence two cubic superpotential terms coupling these to neighbor-
ing bifundamental multiplets. It also has two quartic superpotential terms coupling
bifundamentals charged under $\mathrm{U}\left(N_{2}\right)$, and those charged under $\mathrm{U}\left(N_{3}\right)$. The partition
function of the surface operator inserted by coupling the theory to $N_{f}^{2}$ hypermultiplets is
captured by a Toda CFT correlator with two full punctures at 0 and $\infty$, one simple at 1
and four degenerate punctures at $x_{4}=\hat{z}_{4}, x_{3}=\hat{z}_{4} \hat{z}_{3}, x_{2}=\hat{z}_{4} \hat{z}_{3} \hat{z}_{2}, x_{1}=\hat{z}_{4} \hat{z}_{3} \hat{z}_{2} \hat{z}_{1}$, where
$\hat{z}_{4}=(-1)^{N_{3}+N_{f}} z_{4}, \hat{z}_{3}=(-1)^{N_{2}+N_{4}+N_{3}-1} z_{3}, \hat{z}_{2}=(-1)^{N_{1}+N_{3}+N_{2}-1} z_{2}, \hat{z}_{1}=(-1)^{N_{2}} z_{1}$.
The degenerate punctures are labelled by the $\left(N_{4}-N_{3}\right)$-th symmetric, the $\left(N_{3}-N_{2}\right)$-th
antisymmetric, the $\left(N_{2}-N_{1}\right)$-th symmetric, and the $N_{1}$-th symmetric representations,
depicted by cartoons of their Young diagrams. Whenever two neighboring punctures
have a different type of representation the corresponding gauge theory node has no
adjoint, while neighbors of the same type yield an adjoint. The end node $\mathrm{U}\left(N_{4}\right)$ is
special and has an adjoint because the first puncture is symmetric.

Figure 1. Example of how multiple Toda CFT degenerate punctures map to a quiver gauge theory.

the fundamental weights of $A_{N_{f}-1}$ transform as $\left(\omega_{N}\right)^{C}=\omega_{N_{f}-N}$. We also establish these dualities through explicit evaluation of the exact two-sphere partition function $[17,18]$ of dual theories. This completes the dictionary between symmetries of Toda CFT correlators and dualities of $4 \mathrm{~d} / 2 \mathrm{~d}$ gauge theories (see table 3 ). 


\begin{tabular}{|c|c|c|c|c|}
\hline Duality & Quiver & $W$ & Dual parameters & Toda symmetry \\
\hline Seiberg & & 0 & $\begin{array}{l}N^{D}=N_{f}-N, z^{D}=(-1)^{\widetilde{N_{f}}} z \\
\mathrm{q}^{D}=1-\mathrm{q}, \mathrm{m}^{D}=-\mathrm{m}\end{array}$ & $\begin{array}{l}\text { Conjugation p. } 51 \\
\left(-b \omega_{N}\right)^{C}=-b \omega_{N^{D}}\end{array}$ \\
\hline$(2,2)^{*}$-like & & & $\begin{array}{l}N^{D}=\sum_{t} l_{t}-N, z^{D}=z^{-1} \\
\mathrm{q}^{D}=\mathrm{q}, \mathrm{m}^{D}=\mathrm{m}\end{array}$ & $\begin{array}{l}\text { Crossing p. } 56 \\
\text { simple } \rightarrow \text { degenerate }\end{array}$ \\
\hline $\begin{array}{l}\text { Kutasov-- } \\
\text { Schwimmer }\end{array}$ & & $\operatorname{Tr} X^{l-}$ & $\begin{array}{l}N^{D}=l N_{f}-N \\
z^{D}=(-1)^{N_{f}-\widetilde{N_{f}}} z \\
\mathrm{q}^{D}=\frac{2}{l+1}-\mathrm{q}, \mathrm{m}^{D}=-\mathrm{m}\end{array}$ & $\begin{array}{l}\text { Conjugation } \begin{array}{r}\text { p. } 59 \\
\left(-N b h_{1}\right)^{C} \equiv-N^{D} b h_{1}\end{array}\end{array}$ \\
\hline Quiver & & & $\begin{array}{l}N_{j}^{D}=N_{j-1}+N_{j+1}-N_{j} \\
\hat{z}_{j}^{D}=\hat{z}_{j}^{-1}, \hat{z}_{j \pm 1}^{D}=\hat{z}_{j} \hat{z}_{j \pm 1}\end{array}$ & $\begin{array}{l}\text { Crossing } \quad \text { p. } 63 \\
\omega_{N_{j}-N_{j-1}} \leftrightarrow \omega_{N_{j+1}-N_{j}}\end{array}$ \\
\hline Quiver & & & $\begin{array}{l}N_{j}^{D}=j N_{f}-N_{j} \forall j \\
\mathrm{q}^{D}=1-\mathrm{q}, \mathrm{m}^{D}=-\mathrm{m}\end{array}$ & $\begin{array}{l}\text { Conjugation p. } 66 \\
\omega_{N_{j}-N_{j-1}}^{C}=\omega_{N_{j}^{D}-N_{j-1}^{D}}\end{array}$ \\
\hline
\end{tabular}

Table 2. Dualities of $\mathcal{N}=(2,2)$ quiver gauge theories realized as symmetries in the Toda CFT. Chiral multiplets are denoted by $q_{t}$ (fundamentals), $\widetilde{q}_{t}$ (antifundamentals), and $X$ (adjoint). Each has a twisted mass $\mathrm{m}$ and an $R$-charge $\mathrm{q}$. FI and theta parameters combine into $z=e^{-2 \pi \xi+\mathrm{i} \vartheta}$ for each gauge group $\mathrm{U}(N)$; denoting $n_{f}$ and $n_{a}$ the numbers of fundamental and of adjoint chiral multiplets, we also define $\hat{z}=(-1)^{n_{f}+\left(n_{a}-1\right)(N-1)} z$. For Seiberg and Kutasov-Schwimmer dualities, the magnetic theory contains extra free chiral multiplets whose charges are identical to those of mesons in the electric theory. We assume $\widetilde{N_{f}}<N_{f}$.

\begin{tabular}{|c|c|c|}
\hline Toda CFT move & $\Longleftrightarrow$ & Gauge theory duality \\
\hline $\begin{array}{c:c}\dot{1} & \vdots \\
\dot{r} & \dot{1}\end{array}$ & $\Longleftrightarrow$ & 4d generalized S-duality [13]; [21] \\
\hline 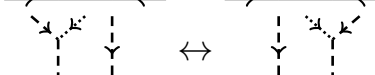 & $\Longleftrightarrow$ & 4d/2d node-hopping [42] \\
\hline 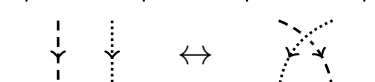 & $\Longleftrightarrow$ & 2d flop transition $[47] ;[18]$ \\
\hline$\underset{\rightarrow}{i}:$\begin{tabular}{l}
$\vdots$ \\
\hdashline
\end{tabular} & $\Longleftrightarrow$ & $\begin{array}{l}\text { 2d Seiberg duality [17]: our section } 3.1 \\
\text { Kutasov-Schwimmer: our section } 3.2\end{array}$ \\
\hline 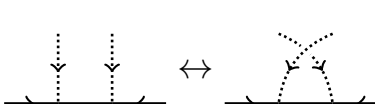 & $\Longleftrightarrow$ & $\begin{array}{l}2 \mathrm{~d} \text { Seiberg and }(2,2)^{*} \text { dualities } \\
\text { for quivers [48]: our section } 3.3\end{array}$ \\
\hline
\end{tabular}

Table 3. The effect of a few Toda CFT moves on the corresponding $4 \mathrm{~d} / 2 \mathrm{~d}$ gauge theory. Besides the symmetry under changing trinion decomposition, Toda CFT correlators are also invariant under conjugation of all momenta. Full punctures are drawn as solid lines, simple punctures as dashed lines, and degenerate punctures as dotted lines. References are to papers describing the gauge theory duality and to papers giving its relation to Toda CFT.

The detailed description of the rest of the paper follows. Section 2 is devoted to the correspondence between surface operators labeled by two dimensional quiver gauge theories and Toda CFT degenerate operators. We derive the identification by coupling 
the two dimensional theories to the trinion theory of free hypermultiplets, as this choice of a free four dimensional theory lets us concentrate on the two dimensional theories. The $S^{2} \subset S_{b}^{4}$ partition function of these surface operators corresponds to Toda CFT correlators involving one simple, two full, and additional degenerate operators.

After describing our gauge theory setup, and recalling explicit expressions for the $S_{b}^{4}$ and $S^{2}$ contributions, we proceed to expand $S^{2}$ partition functions in various limits and compare them with Toda CFT results. First, we review the case of SQED in some detail in section 2.1: this $\mathrm{U}(1)$ gauge theory corresponds to the insertion of the simplest Toda CFT degenerate vertex operator, labeled by the fundamental representation of $A_{N_{f}-1}$ [18]. Then, we move on to $\mathrm{U}(N)$ SQCD in section 2.2, which corresponds to inserting a degenerate operator labeled by an antisymmetric representation of $A_{N_{f}-1}$. Using new braiding matrices derived in appendix A.3, we prove that the Toda CFT correlator and the partition function of the $4 \mathrm{~d} / 2 \mathrm{~d}$ theory are equal. We then describe in section 2.2.3 how one can decouple some free hypermultiplets from the four dimensional theory and chiral multiplets from the two dimensional theory: the procedure translates to a collision limit where two Toda CFT vertex operators combine into an irregular puncture (see also appendix A.6). In section 2.3, we add adjoint matter to SQCD to get SQCDA, and find that it corresponds to a degenerate operator labeled by a symmetric representation. We then consider SQCDA with different superpotentials in section 2.3.3 and give their Toda CFT interpretation. Finally, in section 2.4, we show that the previous results arise as special cases of surface operators described by the quivers (1.8), which correspond to the insertion of several (symmetric and antisymmetric) degenerate operators. We briefly discuss a brane diagram interpretation of the dictionary. By fusing representations, we deduce in section 2.4.2 which surface operator corresponds to an arbitrary degenerate operator. All cases are summarized in table 1.

Section 3 describes dualities of two dimensional $\mathcal{N}=(2,2)$ gauge theories which can be obtained as manifest Toda CFT symmetries. The dualities relate the IR limits of these theories, and we probe them by comparing $S^{2}$ partition functions of proposed duals. The contribution of free hypermultiplets to the partition function of the $4 \mathrm{~d} / 2 \mathrm{~d}$ theory plays little role. We find several Seiberg-like dualities (generalizing the duality found by Hori and Tong [49]) relating theories with similar matter content but different gauge groups (see table 2). The dualities are most clearly seen through the matching with the Toda CFT, but we also show directly in appendix B that the $S^{2}$ partition functions of dual theories are equal. ${ }^{6}$

We start in section 3.1 with the two dimensional analogue of Seiberg duality [50], between $\mathcal{N}=(2,2) \mathrm{U}(N)$ SQCD with $N_{f}$ flavours, and $\mathrm{U}\left(N_{f}-N\right)$ SQCD with $N_{f}$ flavours. The corresponding Toda CFT correlators are simply related by conjugating all momenta. This operation provides us with the precise map of parameters: $N^{D}=N_{f}-N, z^{D}=$ $(-1)^{N_{f}} z$, and $m^{D}=\mathrm{i} / 2-m$ for the complexified twisted masses of every chiral multiplet. ${ }^{7}$ In addition to fundamental and antifundamental chiral multiplets, the $\mathrm{U}\left(N_{f}-N\right)$

\footnotetext{
${ }^{6}$ This was shown previously for SQCD with $N_{f}$ fundamental and $\widetilde{N_{f}} \leq N_{f}-2$ antifundamental chiral multiplets [17], and generalized very recently to arbitrary $\widetilde{N_{f}}$ in [48]. Our proofs follow the same logic but also apply to theories with an adjoint chiral multiplet and a superpotential.

${ }^{7}$ By coupling the flavour symmetry to a constant background vector multiplet, chiral multiplets can be given twisted masses and $R$-charges, which combine into a complex parameter $m$ for each chiral multiplet.
} 
theory involves a free chiral multiplet transforming in the bifundamental representation of the flavour symmetry group $S\left[\mathrm{U}\left(N_{f}\right) \times \mathrm{U}\left(N_{f}\right)\right]$. These free chiral multiplets couple to the charged multiplets through a cubic superpotential, which must have total $R$-charge 2 (complexified twisted mass i) to be supersymmetric. As was also observed recently in [48], the theories differ by a shift in the FI parameter associated to the U(1) flavour symmetry. In section 3.1.2, we deduce Seiberg duality relations between theories with $N_{f}$ fundamental and $\widetilde{N_{f}}<N_{f}$ antifundamental chiral multiplets (with $z^{D}=(-1)^{\widetilde{N_{f}}} z$ ). For this, we let some of the twisted masses of antifundamental multiplets go to infinity and take into account the renormalization of the FI parameter: this limit precisely corresponds to merging the Toda CFT operators inserted at $\infty$ and 1 into an irregular puncture [51].

We then move on in section 3.2 to dualities of $\mathrm{U}(N)$ SQCDA, which has fundamental, antifundamental, and adjoint chiral multiplets. Without further restriction, the theory features no duality. We find two choices of superpotentials for which the theory has a dual description: both dualities appear to be new in two dimensional $\mathcal{N}=(2,2)$ theories.

In section 3.2.1, we consider SQCDA with the superpotential

$$
W=\sum_{t=1}^{N_{f}} \widetilde{q}_{t} X^{l_{t}} q_{t},
$$

described by a choice of $N_{f}$ integers $l_{t} \geq 0$, where $q_{t}, \widetilde{q}_{t}$ and $X$ are the fundamental, antifundamental, and adjoint chiral multiplets. The theory is a simple generalization of $\mathcal{N}=(2,2)^{*}$ SQCD. ${ }^{8}$ The constraint on $R$-charges due to the superpotential translates to a very natural constraint in the Toda CFT language. Namely, the momentum labeling the simple puncture gets fine-tuned to become a degenerate operator, labeled by a symmetric representation of $A_{N_{f}-1}$. The crossing symmetry exchanging these two degenerate vertex operators thus provides us with a duality between two dimensional SQCDA theories with the superpotential (1.10). The $\mathrm{U}\left(N^{D}\right)$ dual theory features the same chiral multiplets and superpotential as the $\mathrm{U}(N)$ theory, with identical complexified twisted masses, $N^{D}=$ $\sum_{t} l_{t}-N$, and $z^{D}=z^{-1}$.

In section 3.2.2, we consider SQCDA with the superpotential

$$
W=\operatorname{Tr} X^{l+1}
$$

for some integer $l \geq 0$, where $X$ is the adjoint chiral multiplet. We find a direct analogue of the four dimensional Kutasov-Schwimmer duality [52, 53]. It turns out that given the superpotential constraint, conjugation maps the (symmetric) degenerate operator describing $\mathrm{U}(N)$ SQCDA to the degenerate operator describing $\mathrm{U}\left(N^{D}\right)$ SQCDA. The dual gauge theory has $N^{D}=l N_{f}-N, z^{D}=z, m_{t}^{D}=m_{X}-m_{t}, \widetilde{m}_{t}^{D}=m_{X}-\widetilde{m}_{t}$, and $m_{X}^{D}=m_{X}$. As in four dimensions $[52,53]$, the dual theory features $l$ additional free chiral multiplets in the bifundamental representation of $S\left[\mathrm{U}\left(N_{f}\right) \times \mathrm{U}\left(N_{f}\right)\right]$, which correspond to mesons of the electric theory. As for SQCD, the limit where twisted masses of some chiral multiplets are

${ }^{8} \mathcal{N}=(2,2)^{*} \mathrm{SQCD}$ is the mass deformation of the $\mathcal{N}=(4,4)$ theory of a $\mathrm{U}(N)$ vector multiplet coupled to $N_{f}$ fundamental hypermultiplets. Its cubic superpotential $W=\sum_{t} \widetilde{q}_{t} X q_{t}$ corresponds to taking all $l_{t}=1$. 
very large yields similar dualities between theories with a different number of fundamental and antifundamental chiral multiplets.

Lastly, we describe dualities of quiver gauge theories in section 3.3. We consider the $\mathrm{U}\left(N_{1}\right) \times \cdots \times \mathrm{U}\left(N_{n}\right)$ quiver theories (1.8) which correspond in the Toda CFT to the insertion of $n$ degenerate vertex operators. Dualities of another type of $\mathcal{N}=(2,2)$ quiver gauge theories were considered recently in [48].

In section 3.3.1 we apply Seiberg duality or the $\mathcal{N}=(2,2)^{*}$ duality (depending on the presence or absence of an adjoint chiral multiplet) to gauge group factors $\mathrm{U}\left(N_{j}\right)$ with $j<n$. We show that the duality translates to the exchange of degenerate punctures numbered $j$ and $j+1$ in the Toda CFT. Each permutation of the $n$ degenerate punctures is thus realized as a combination of such Seiberg and $\mathcal{N}=(2,2)^{*}$ dualities.

Based on this geometric realization of dualities for $j<n$, we construct in section 3.3.2 the full set of dual theories obtained through Seiberg and $\mathcal{N}=(2,2)^{*}$ dualities acting on any gauge group. We find no Toda CFT description of the duality acting on $\mathrm{U}\left(N_{n}\right)$, except when all degenerate vertex operators are labeled by antisymmetric representations of $A_{N_{f}-1}$. Then, conjugating all Toda CFT momenta yields a different set of degenerate operators of the same type, and it turns out that the corresponding dual gauge theories are related by a sequence of Seiberg and $\mathcal{N}=(2,2)^{*}$ dualities on all nodes. A particular case is the quiver (1.1) which corresponds to a single degenerate vertex operator labeled by an arbitrary representation $\mathcal{R}$ : applying the same sequence of Seiberg and $\mathcal{N}=(2,2)^{*}$ dualities corresponds to conjugating $\mathcal{R}$ and all Toda CFT momenta. This result concludes the description of dualities of two dimensional $\mathcal{N}=(2,2)$ gauge theories which correspond to manifest symmetries of the Toda CFT.

Many new Toda CFT results are presented in appendix A. We describe notations and the normalization of vertex operators (appendix A.1), compare one-loop determinants and three-point functions (appendix A.2), work out new braiding matrices (appendix A.3), give new fusion rules (appendix A.4), deduce new conformal blocks from the correspondence (appendix A.5), and collide vertex operators to build irregular punctures of the $W_{N_{f}}$ algebra (appendix A.6). Finally, appendix B features analytic proofs that vortex partition of dual theories are equal, for Seiberg duality (appendix B.1), and for dualities of SQCD with an adjoint (appendix B.2).

\section{Surface operators as Toda degenerate operators}

In this section, we consider half-BPS surface operators obtained by coupling two dimensional $\mathcal{N}=(2,2)$ gauge theories to four dimensional $\mathcal{N}=2$ theories of class $\mathrm{S}$. We enrich the dictionary between class $\mathrm{S}$ theories and Riemann surfaces by identifying surface operators which correspond to the insertion of arbitrary degenerate punctures.

To make the two dimensional features most visible, we restrict ourselves to surface operators in the simplest class $\mathrm{S}$ theory, which is the theory on $N_{f}$ M5-branes wrapping a sphere with two full and one simple puncture, namely the theory of $N_{f}^{2}$ free hypermultiplets $\Phi^{4 \mathrm{~d}}$. The M-theory description makes an $\mathrm{SU}\left(N_{f}\right) \times \mathrm{SU}\left(N_{f}\right) \times \mathrm{U}(1)$ flavour symmetry manifest, and the hypermultiplets transform in the trifundamental representation of this 
group. All two dimensional theories we study contain $N_{f}$ fundamental chiral multiplets $q$ and $N_{f}$ antifundamental chiral multiplets $\widetilde{q}$ of a $\mathrm{U}(N)$ gauge group factor. The $4 \mathrm{~d} / 2 \mathrm{~d}$ coupling takes the form of a superpotential term $\sum_{s, t} \widetilde{q}_{t} q_{s}\left(\left.\Phi_{s t}^{4 \mathrm{~d}}\right|_{2 \mathrm{~d}}\right)$ in two dimensions, which identifies the flavour symmetries $S\left[\mathrm{U}\left(N_{f}\right) \times \mathrm{U}\left(N_{f}\right)\right]$ of these chiral multiplets ${ }^{9}$ and of the hypermultiplets. To write the superpotential term explicitly, the four dimensional $\mathcal{N}=2$ hypermultiplets should be decomposed into two dimensional $\mathcal{N}=(2,2)$ components. Coupling the common flavour group to a constant background vector multiplet then gives twisted masses to the two dimensional chiral multiplets and masses to the four dimensional hypermultiplets, related by (2.17).

For definiteness, we place the four dimensional theory on a squashed sphere $S_{b}^{4}$

$$
\frac{x_{0}^{2}}{r}+\frac{x_{1}^{2}+x_{2}^{2}}{\ell^{2}}+\frac{x_{3}^{2}+x_{4}^{2}}{\tilde{\ell}^{2}}=1
$$

where $b^{2}=\ell / \tilde{\ell}$, and we place surface operators at $x_{3}=x_{4}=0$, hence on the squashed two-sphere ${ }^{10}$

$$
\frac{x_{0}^{2}}{r}+\frac{x_{1}^{2}+x_{2}^{2}}{\ell^{2}}=1 .
$$

The full partition function of the $4 \mathrm{~d} / 2 \mathrm{~d}$ theory is then the product

$$
Z_{S^{2} \subset S_{b}^{4}}=Z_{S_{b}^{4}}^{\text {free }} Z_{S^{2}}
$$

of the partition functions of the free hypermultiplets on $S_{b}^{4}[15]$ and of the two dimensional gauge theory on the squashed two-sphere [17-19]. The two factors do not dependent on $r$, but only on the equatorial radii $\ell$ and $\tilde{\ell}$.

The $S_{b}^{4}$ partition function of a single free hypermultiplet of mass $m$ only depends on the dimensionless mass ${ }^{11} m=\sqrt{\ell \tilde{\ell}} \mathrm{m}$. It reads $[15]^{12}$

$$
Z_{S_{b}^{4}}^{\mathrm{free}}(\mathrm{m})=\frac{1}{\Upsilon\left(\frac{b}{2}+\frac{1}{2 b}-\mathrm{i} m\right)} .
$$

The $S_{b}^{4}$ partition function of the four dimensional theory is the product of $N_{f}^{2}$ such inverses of Upsilon functions. The complexified masses $m_{s t}$ of the $N_{f}^{2}$ hypermultiplets in this class $\mathrm{S}$ theory arise from coupling to a background vector multiplet the $S\left[\mathrm{U}\left(N_{f}\right) \times \mathrm{U}\left(N_{f}\right)\right]$ flavour subgroup which is made manifest in the description as M5-branes wrapping a trinion. With such masses, the $S_{b}^{4}$ partition function is then equal to a Toda CFT correlator with one simple and two full punctures. Inserting one or more degenerate punctures in the correlator corresponds to including the associated surface operator in the theory of $N_{f}^{2}$ hypermultiplets: for given degenerate punctures, we will find the gauge theory description of

\footnotetext{
${ }^{9}$ The full flavour symmetry of the two dimensional quiver gauge theories we consider also contains a $\mathrm{U}(1)$ factor, under which adjoint chiral multiplets have charge \pm 2 and bifundamental chiral multiplets have charge $\mp 1$.

${ }^{10}$ Inserting the surface operators at $x_{1}=x_{2}=0$ instead would exchange $\ell \leftrightarrow \tilde{\ell}$ : we would find degenerate operators with momenta $-\frac{1}{b} \Omega$ instead of $-b \Omega$, where $\Omega$ is a highest weight of $A_{N_{f}-1}$.

${ }^{11}$ In our correspondence $m$ also has an imaginary part, which is linked to the $\mathrm{U}(1) R$-charges of the two dimensional chiral multiplets.

${ }^{12}$ The sign of $m$ is irrelevant since the Upsilon function (A.9) obeys $\Upsilon\left(b+\frac{1}{b}-x\right)=\Upsilon(x)$.
} 
the associated surface operator by comparing the enriched Toda CFT correlator with the partition function of the $4 \mathrm{~d} / 2 \mathrm{~d}$ theory on $S^{2} \subset S_{b}^{4}$.

The second contribution to the partition function of the $S^{2} \subset S_{b}^{4}$ system is the partition function of the two dimensional theory. We recall now the data defining an $\mathcal{N}=(2,2)$ theory of vector and chiral multiplets, and expressions for its partition function on $S^{2}$. Besides the gauge group $G$ (throughout the paper, $G=\mathrm{U}(N)$ or a product of such factors) and the representation $R$ of $G$ in which matter multiplets transform, the $S^{2}$ partition function depends on a (real) twisted mass $\mathrm{m}$ and a $\mathrm{U}(1) R$-charge $\mathrm{q}$ for each chiral multiplet, that is, for each irreducible factor in $R$. Those are conveniently combined as the dimensionless complexified twisted mass

$$
m=\ell \mathrm{m}+\frac{\mathrm{iq}}{2},
$$

where $\ell$ is the equatorial radius of the squashed $S^{2}$. Furthermore, for each $\mathrm{U}(1)$ factor of $G$, an FI parameter $\xi$ and a theta angle $\vartheta$ can be turned on. It will be practical to consider the complex combination

$$
z=e^{-2 \pi \xi+\mathrm{i} \vartheta}
$$

for each $\mathrm{U}(1)$ gauge group factor. Unless stated otherwise, the parameters $m$ and $z$ are generic. We also assume that $R$-charges are small and positive, $0<\operatorname{Re}(-2 \mathrm{i} m)<1$, and otherwise define the partition function by analytic continuation.

For a choice of supercharge $\mathcal{Q}$ in the supersymmetry algebra, and of a $\mathcal{Q}$-exact deformation term $\mathcal{Q} V$ such that $\mathcal{Q}^{2} V=0$, supersymmetric localization reduces the partition function to an integral over saddle points of $\mathcal{Q} V$. When $\mathcal{Q} V$ is chosen appropriately, in particular with a positive semidefinite bosonic part, the integral is finite dimensional and more tractable than the original path integral.

One choice of deformation term leads to an expression of the partition function as an integral over the Coulomb branch $[17,18]\left([54,55]\right.$ corrected a sign): ${ }^{13}$

$Z=\frac{1}{\mathcal{W}} \sum_{B \in \mathfrak{h}_{\mathbb{Z}}} \int_{\mathfrak{h}} \frac{\mathrm{d} \sigma}{(2 \pi)^{\mathrm{dim} \mathfrak{h}}} Z_{\mathrm{cl}} \prod_{\alpha>0}\left[(-1)^{\alpha B}\left((\alpha \sigma)^{2}+\frac{(\alpha B)^{2}}{4}\right)\right] \prod_{w \in R}\left[\frac{\Gamma\left(-w\left(\mathrm{i} m+\mathrm{i} \sigma+\frac{B}{2}\right)\right)}{\Gamma\left(1+w\left(\mathrm{i} m+\mathrm{i} \sigma-\frac{B}{2}\right)\right)}\right]$.

Here, $\mathcal{W}$ is the order of the Weyl group of $G$, the sum is restricted to GNO quantized fluxes $B \in \mathfrak{h}$, and the integral over the lowest component $\sigma$ of the vector multiplet ranges in the Cartan algebra $\mathfrak{h}$ of $G$. The vector multiplet one-loop determinant is a product over all positive roots $\alpha$ of $G$, and the chiral multiplet one-loop determinant, a product over all weights $w$ of $R$, involves the complexified twisted mass $w \cdot m$ of the irreducible factor of $R$ containing $w \cdot{ }^{14}$ When $G=\mathrm{U}\left(N_{1}\right) \times \cdots \times \mathrm{U}\left(N_{n}\right)$, the classical contribution is

$$
Z_{\mathrm{cl}}(\sigma, B, z, \bar{z})=\prod_{i=1}^{n}\left[z_{i}^{\operatorname{Tr}\left(\mathrm{i} \sigma_{i}+\frac{B_{i}}{2}\right)} \bar{z}_{i}^{\operatorname{Tr}\left(\mathrm{i} \sigma_{i}-\frac{B_{i}}{2}\right)}\right]=\prod_{i=1}^{n} e^{-4 \pi \xi_{i} \operatorname{Tr}\left(\mathrm{i} \sigma_{i}\right)+\mathrm{i} \vartheta_{i} \operatorname{Tr}\left(B_{i}\right)},
$$

and it is invariant under any $\vartheta_{i} \rightarrow \vartheta_{i}+2 \pi$ since $B_{i}$ are $N_{i} \times N_{i}$ (diagonal) matrices of integers. The vector multiplet sign simply shifts $\vartheta_{i} \rightarrow \vartheta_{i}+\left(N_{i}-1\right) \pi$.

\footnotetext{
${ }^{13}$ Our normalization differs by $(2 \pi)^{\operatorname{dim} \mathfrak{h}}$ from [18] as this will simplify the expression of dualities.

${ }^{14}$ Roots and weights are linear forms on $\mathfrak{h}$, and we use the notation $\alpha \sigma=\alpha(\sigma) \in \mathbb{R}$.
} 
A different choice of deformation term $[17,18]$ localizes the path integral to the Higgs branch of the theory rather than its Coulomb branch, yielding a finite sum

$$
Z=\sum_{v \in\{\text { Higgs vacua }\}}(z \bar{z})^{\mathrm{i} v} \underset{\sigma=v}{\operatorname{res}}\left[\prod_{\alpha}(\mathrm{i} \alpha \sigma) \prod_{w \in R} \gamma(-w(\mathrm{i} m+\mathrm{i} \sigma))\right] Z_{\mathrm{v}}(v, m, z) Z_{\overline{\mathrm{v}}}(v, m, \bar{z})
$$

which includes a vortex contribution $Z_{\mathrm{v}}$ depending holomorphically on $z$ and an antivortex contribution depending on $\bar{z}$. Here, $\gamma(x)=\frac{\Gamma(x)}{\Gamma(1-x)}$, and factors other than $Z_{\mathrm{v}}$ and $Z_{\overline{\mathrm{v}}}$ are obtained as the residue at $\sigma=v$ and $B=0$ of the Coulomb branch integrand. Higgs branch vacua are defined as having non-zero vevs for the lowest component $\phi$ of some chiral fields. They are labeled by solutions $(\sigma, \phi)$ of the D-term equation $\phi \phi^{\dagger}=\xi$ and of $(\sigma+m) \phi=0$, where $\sigma$ and $m$ act on $\phi$ through the action of $G$ and of the flavour symmetry group $G_{f}$. The set of values of $\sigma$ for which the D-term equation has a solution depends on signs of the FI parameters $\xi_{j}$ for each $\mathrm{U}(1)$ factor in $G$ : each choice of signs leads to a different expansion (2.9). Even after solving these equations, one must in principle evaluate $Z_{\mathrm{v}}$ as the volume of a moduli space of vortices. However, the Coulomb branch representation provides a convenient short-cut: closing the $\mathrm{d} \sigma$ integrals (2.7) towards $\sigma \rightarrow \pm \mathrm{i} \infty$ depending on the matter content and on signs of FI parameters expresses the partition function as a sum over poles, which is then rewritten as a finite sum of factorized terms (2.9). The manipulations are most easily done on specific examples, as we will see, but work for an arbitrary gauge group and matter representation (see [18, appendix F]).

In the coming sections we associate a two dimensional $\mathcal{N}=(2,2)$ gauge theory, hence a surface operator, to each choice of representation $\mathcal{R}(\Omega)$ of $A_{N_{f}-1}$. We work out equalities of the form

$$
Z_{S^{2} \subset S_{b}^{4}}^{(\Omega)}=A|x|^{2 \gamma_{0}}|1-x|^{2 \gamma_{1}}\left\langle\widehat{V}_{\alpha_{\infty}}(\infty) \widehat{V}_{\hat{m}}(1) \widehat{V}_{\alpha_{0}}(0) \widehat{V}_{-b \Omega}(x, \bar{x})\right\rangle
$$

between the partition function on $S^{2} \subset S_{b}^{4}$ of the $4 \mathrm{~d} / 2 \mathrm{~d}$ system associated to a given representation $\mathcal{R}(\Omega)$ and Toda CFT correlators with two full punctures at 0 and $\infty$, one simple at 1 , and one degenerate. ${ }^{15}$ The position $x$ of the degenerate puncture is related to a complexified FI parameter $z$. The two dimensional theories we consider involve $N_{f}$ fundamental and $N_{f}$ antifundamental chiral multiplets of a gauge group factor $\mathrm{U}\left(N_{n}\right)$, whose twisted masses we denote by $m_{t}$ and $\widetilde{m}_{t}$.

Let us first explain how the factor $A|x|^{2 \gamma_{0}}|1-x|^{2 \gamma_{1}}$ can be absorbed into the partition function (specifically the $S^{2}$ contribution). In the coming sections it will be easier to manipulate explicit expressions of partition functions and correlators, hence we will keep the factor explicitly, with the understanding that it has no physical content. In terms of

\footnotetext{
${ }^{15}$ Toda CFT notations are reviewed in appendix A.1. Vertex operators $\widehat{V}_{\alpha}$ are labeled by their momentum $\alpha$, a linear combination of the weights $h_{s}\left(1 \leq s \leq N_{f}\right)$ of the fundamental representation of $A_{N_{f}-1}$. They are primary operators for the $W_{N_{f}}$ chiral algebra. Generic momenta depend on $N_{f}-1$ parameters and label full punctures. Semi-degenerate vertex operators, with momentum $\varkappa h_{1}$ (or its conjugate $-\varkappa h_{N_{f}}$ ), have null descendants under $W_{N_{f}}$ and label simple punctures. Degenerate vertex operators have momentum $-b \Omega-\Omega^{\prime} / b$ for a pair of highest weights $\Omega$ and $\Omega^{\prime}$ of representations of $A_{N_{f}-1}$.
} 
gauge theory data, it turns out that we can split

$$
\gamma_{0}=\gamma_{0}^{\circ}(\Omega, b)-\frac{N_{n}}{N_{f}} \sum_{t=1}^{N_{f}} \mathrm{i} m_{t}, \quad \gamma_{1}=\gamma_{1}^{\circ}(\Omega, b)+\frac{N_{n}}{N_{f}} \sum_{t=1}^{N_{f}}\left(\mathrm{i} m_{t}+\mathrm{i} \widetilde{m}_{t}\right),
$$

and $A=A^{\circ}(\Omega, b) b^{-2 N_{n} \sum_{t}\left(\mathrm{i} m_{t}+\mathrm{i} \tilde{m}_{t}\right)}$, where $A^{\circ}, \gamma_{0}^{\circ}$ and $\gamma_{1}^{\circ}$ depend only on $b$ and $\Omega$. The factor decomposes as

$$
A|x|^{2 \gamma_{0}}|1-x|^{2 \gamma_{1}}=\left[A^{\circ}|x|^{2 \gamma_{0}^{\circ}}|1-x|^{2 \gamma_{1}^{\circ}}\right]\left[|x|^{-2\left(N_{n} / N_{f}\right) \sum_{t} \mathrm{i} m_{t}}\right]\left[\frac{|1-x|^{2 N_{n}}}{b^{2 N_{n} N_{f}}}\right]^{\sum_{t}\left(\mathrm{i} m_{t}+\mathrm{i} \tilde{m}_{t}\right) / N_{f}}
$$

and can be absorbed in the partition function through three different mechanisms. Firstly, the two-sphere partition function is subject to certain ambiguities [19] (see also [56]). These are captured by local supergravity counterterms [57]. A change in the renormalization scheme changes the partition function by

$$
Z \rightarrow f(z) \bar{f}(\bar{z}) Z
$$

where $f$ is a holomorphic function of the complexified FI parameter $z$. This lets us absorb the first factor in (2.12) as a renormalization ambiguity of $Z_{S^{2} \subset S_{b}^{4}}$. Secondly, a constant $\mathrm{U}(1)$ gauge transformation lets us shift the partition function by any power of $|z|^{2}=$ $|x|^{2}$ hence absorb the second factor in (2.12). Finally, the third factor can be absorbed through a complexified FI parameter $z_{\mathrm{fl}}=b^{2 N_{n} N_{f}} /(1-x)^{2 N_{n}}$ for the U(1) subgroup of the flavour group $S\left[\mathrm{U}\left(N_{f}\right) \times \mathrm{U}\left(N_{f}\right)\right]$ which acts on the fundamental and antifundamental chiral multiplets. Indeed, such an FI parameter multiplies the partition function by $\left(z_{\mathrm{fl}} \bar{z}_{\mathrm{fl}}\right)^{\mathrm{i} \sigma_{\mathrm{fl}}}$, where $\sigma_{\mathrm{ff}}$ is the bottom component of the background vector multiplet coupled to the $\mathrm{U}(1)$ flavour symmetry, that is, $\sigma_{\mathrm{fl}}=\sum_{t}\left(m_{t}+\widetilde{m}_{t}\right) /\left(2 N_{f}\right)$.

We are now ready to discuss how we will derive equalities of the form (2.10), or more generally for a set of highest weights $\Omega_{j}$ of $A_{N_{f}-1}$ :

$$
Z_{S^{2} \subset S_{b}^{4}}^{\left\{\Omega_{j}\right\}}=\left\langle\widehat{V}_{\alpha_{\infty}}(\infty) \widehat{V}_{\hat{m}}(1) \widehat{V}_{\alpha_{0}}(0) \prod_{j=1}^{n} \widehat{V}_{-b \Omega_{j}}\left(x_{j}, \bar{x}_{j}\right)\right\rangle
$$

where $\alpha_{0}$ and $\alpha_{\infty}$ are generic, $\hat{m}$ is semi-degenerate, and we have omitted the factors which can be absorbed into the partition function. The dictionary between gauge theory and Toda CFT data identifies the momenta $\alpha_{0}, \alpha_{\infty}$, and $\hat{m}$ to the three factors of the flavour symmetry group $\mathrm{SU}\left(N_{f}\right) \times \mathrm{SU}\left(N_{f}\right) \times \mathrm{U}(1)$ acting on fundamental and antifundamental chiral multiplets:

$$
\begin{aligned}
\alpha_{0}=Q-\frac{1}{b} \sum_{s=1}^{N_{f}} \mathrm{i} m_{s} h_{s}, & \hat{m}=(\varkappa+N b) h_{1}, \\
\alpha_{\infty}=Q-\frac{1}{b} \sum_{s=1}^{N_{f}} \mathrm{i} \widetilde{m}_{s} h_{s}, & \varkappa=\frac{1}{b} \sum_{s=1}^{N_{f}}\left(1+\mathrm{i} m_{s}+\mathrm{i} \widetilde{m}_{s}\right),
\end{aligned}
$$


with Toda CFT notations given in appendix A.1. The degenerate operators encode the choice of gauge groups and matter content of the gauge theory.

As explained below, we will start in each case by matching the dependence of the $S^{2}$ partition function on FI parameters $z_{j}$ with the dependence of Toda CFT correlators on the position of degenerate operators $x_{j}$. Once this is done, there remains a universal relative factor between the $S^{2}$ partition function and the Toda CFT correlator, which turns out to be a Toda CFT three-point function of two generic and one semi-degenerate vertex operators $^{16}$

$$
\widehat{C}\left(\alpha_{0}, \alpha_{\infty}, \varkappa h_{1}\right)=\prod_{s=1}^{N_{f}} \prod_{t=1}^{N_{f}} \frac{1}{\Upsilon\left(\frac{1}{b}\left(1+\mathrm{i} m_{s}+\mathrm{i} \tilde{m}_{t}\right)\right)} .
$$

These Upsilon functions are precisely reproduced by the $S_{b}^{4}$ partition function (2.4) of $N_{f}^{2}$ free hypermultiplets with (dimensionless) masses

$$
m_{s t}=\mathrm{i} \frac{1-b^{2}}{2 b}-\frac{1}{b}\left(m_{s}+\widetilde{m}_{t}\right) .
$$

The dimensionful masses $(\ell \tilde{\ell})^{\frac{-1}{2}} m_{s t}$ and twisted masses $\ell^{-1} m_{s}$ and $\ell^{-1} \widetilde{m}_{t}$ both originate from coupling the common flavour symmetry group $\mathrm{SU}\left(N_{f}\right) \times \mathrm{SU}\left(N_{f}\right) \times \mathrm{U}(1)$ to a background vector multiplet, and indeed, the relation between dimensionful masses has no relative factor of $b$ :

$$
\left[\frac{m_{s t}}{\sqrt{\ell \tilde{\ell}}}+\frac{\mathrm{i}}{2 \ell}+\frac{\mathrm{i}}{2 \tilde{\ell}}\right]+\frac{m_{s}+\widetilde{m}_{t}}{\ell}=\frac{\mathrm{i}}{\ell}
$$

The masses $m_{s t}$ can also be found by requiring that the two dimensional superpotential $\left.\sum_{s, t} \widetilde{q}_{t} q_{s} \Phi_{s t}^{4 \mathrm{~d}}\right|_{2 \mathrm{~d}}$ is supersymmetric hence has $R$-charge 2 (complexified twisted mass i). From this perspective, the shift in the four dimensional masses likely arises from mixing the $\mathrm{U}(1)_{R}$ symmetry with geometrical symmetries.

In section 2.1 and section 2.2, we identify degenerate vertex operators labeled by the fundamental (resp. antisymmetric) representation of $A_{N_{f}-1}$ to SQED (resp. SQCD). The Toda CFT correlator is a four-point function which depends on a single cross-ratio $x$, while the two dimensional theory has a single $U(1)$ gauge group factor hence a single complexified FI parameter $z=e^{-2 \pi \xi+\mathrm{i} \vartheta}$. We prove as follows that the Toda correlator is equal to the $S^{2} \subset S_{b}^{4}$ partition function. First, we write the Higgs branch expressions of the $S^{2}$ partition function in the regions $\xi>0$ and $\xi<0$, that is, $|z|<1$ and $|z|>1$. The two expressions match with expansions of the Toda CFT correlator in the s-channel $|x|<1$ and u-channel $|x|>1$ as described in table 4: the Higgs branch vacua correspond to choices of internal momenta and we match the leading powers of $|z|^{2}=|x|^{2}$. On the gauge theory side, the exponents of $|z|^{2}$ are read from the classical contribution, while on the Toda CFT side the exponents of $|x|^{2}$ are sums of dimensions of vertex operators. We then

\footnotetext{
${ }^{16}$ Note the shift between $\varkappa h_{1}$ in the three-point function (2.16) and $\hat{m}$ in the $(n+3)$-point function $(2.14)$. The insertion of degenerate operators near a simple puncture thus shifts the dictionary between the semidegenerate momentum of the puncture and the corresponding hypermultiplet mass. As a result, the nodehopping duality relates surface operators in four dimensional theories which differ by shifts in complexified masses of hypermultiplets.
} 


\begin{tabular}{|cll|}
\hline & Gauge theory & Toda CFT \\
\hline Terms in the sum & Higgs vacua & Internal momenta \\
Asymptotics at 0 & Classical contribution $(z \bar{z})^{\mathrm{i} v}$ & $(x \bar{x})^{\Delta\left(\alpha_{0}-b h\right)-\Delta\left(\alpha_{0}\right)-\Delta(-b \omega)}$ \\
Leading coefficient & One-loop determinant $Z_{11}$ & Three-point functions \\
Holomorphic series & Vortex partition function $Z_{\mathrm{v}}$ & Conformal blocks (normalized) \\
\hline
\end{tabular}

Table 4. Relation between parts of the Higgs branch decomposition of the $S^{2}$ partition function, and the s-channel decomposition of corresponding Toda CFT correlators. Explicit expressions differ by $A|x|^{2 \gamma_{0}}|1-x|^{2 \gamma_{1}}$, an ambiguity in $Z$.

derive the braiding matrices which relate s-channel and $\mathrm{u}$-channel conformal blocks and show that they are equal to the corresponding gauge theory data. These braiding matrices let us express the monodromy around $\infty$ as a matrix in the basis of s-channel conformal blocks (the monodromy around 0 is diagonal in this basis). Finally, we prove that the $S^{2}$ partition function has only one branch point besides $z=0$ and $z=\infty$, and identify gauge theory exponents with those in the t-channel $x \rightarrow 1$ of the Toda correlator. Therefore the monodromy matrix around 1 is simply the inverse product of the monodromies around 0 and $\infty$. Since their monodromy matrices around all three branch points coincide, the $S^{2}$ partition function and Toda CFT four-point function must be equal up to a factor with no monodromy. Since in expansions around 0,1 and $\infty$ the exponents match, the factor has no pole on the sphere hence is a constant: it is precisely given by the $S_{b}^{4}$ contribution (2.16) of $N_{f}^{2}$ hypermultiplets.

When the FI parameter $\xi$ is changed continuously from $\xi<0$ to $\xi>0$, the two dimensional gauge theory experiences a flop transition between vortices carried by fundamental matter and vortices carried by antifundamental matter. The flop transition is realized in the Toda CFT as crossing symmetry from the s-channel to the u-channel [18]. This geometric approach implies that the results for $\xi<0$ and $\xi>0$ are related by analytic continuation. There is no Higgs branch expansion as $\xi \rightarrow 0$ : instead, we build a decomposition of the Coulomb branch integral in this limit. It would be interesting to provide a gauge theory interpretation of this "t-channel" decomposition, and of the braiding matrices relating $\xi>0$ and $\xi<0$ vortex partition functions.

In section 2.3 , we identify degenerate vertex operators labeled by symmetric representations of $A_{N_{f}-1}$ to SQCD with an additional adjoint chiral multiplet (SQCDA). The discussion is very similar to the previous cases, but braiding matrices are not available. ${ }^{17}$ Instead, we check that the leading coefficients and powers of $|x|^{2}$ coincide, both in the s-channel and in the u-channel. We then check that the $S^{2}$ partition function has a branch point corresponding to the t-channel, and that the leading powers of $|1-x|^{2}$ coincide. As before, the Toda CFT four-point function is equal to the $S^{2}$ partition function up to a constant, which is the $S_{b}^{4}$ partition function of $N_{f}^{2}$ free hypermultiplets.

\footnotetext{
${ }^{17}$ It is technically difficult to write down braiding matrices in this case. On the gauge theory side, the Mellin-Barnes integral (used for SQED and SQCD to interpolate between $|z| \lessgtr 1$ expansions) is much more involved. On the Toda CFT side, recursion relations for the braiding matrices contain many more terms than for the antisymmetric case.
} 
In section 2.4 we identify the quiver gauge theory which corresponds to sets of degenerate operators labeled by symmetric or antisymmetric representations of $A_{N_{f}-1}$. The identification is checked by comparing the expansion of the $S^{2} \subset S_{b}^{4}$ partition function and of the Toda CFT correlator in various limits. Seiberg-like dualities let us probe further limits: as seen in section 3.3.1, permutations of the $n$ degenerate vertex operators relate dual gauge theories. First, we equate exponents and leading coefficients in the channel where degenerate punctures are at $0<\left|x_{1}\right|<\cdots<\left|x_{n}\right|<1$. Thanks to dualities, exponents and leading coefficients also match for all other orderings of the $n$ degenerate punctures. By symmetry, the gauge theory and Toda CFT exponents and leading coefficients also match in all channels with $1<\left|x_{1}\right|, \ldots,\left|x_{n}\right|<\infty$. In each of the $2(N$ !) channels the decompositions involve many exponents and factors, and all match. We then equate exponents which appear in any of the limits $x_{n} \rightarrow 1$ or $x_{j} \rightarrow x_{j+1}$, hence also in the limits $x_{j} \rightarrow 1$ or $x_{j} \rightarrow x_{k}$ thanks to dualities.

Building upon the identification of the quiver which corresponds to the insertion of any number of antisymmetric degenerate vertex operators, we show in section 2.4.2 that bringing all punctures $x_{j}=x$ to the same position yields a degenerate vertex operator labeled by an arbitrary representation of $A_{N_{f}-1}$ : all other terms in the fusion of antisymmetric degenerate vertex operators appear with higher powers of some $\left|x_{j}-x_{k}\right|^{2}$ hence are suppressed. This determines the quiver gauge theory corresponding to an arbitrary degenerate vertex operator $\widehat{V}_{-b \Omega}$.

The surface operators we consider are constructed by coupling $N_{f}$ fundamental and $N_{f}$ antifundamental chiral multiplets of an $\mathcal{N}=(2,2)$ theory to $N_{f}^{2}$ hypermultiplets. Making some antifundamental chiral multiplets and some hypermultiplets massive yields surface operators described by $\mathcal{N}=(2,2)$ theories with $N_{f}$ fundamental and $\widetilde{N_{f}}<N_{f}$ antifundamental chiral multiplets, coupled to $N_{f} \widetilde{N}_{f}$ free hypermultiplets. The limit corresponds to a collision limit of the punctures $\widehat{V}_{\hat{m}}$ and $\widehat{V}_{\alpha_{\infty}}$ in (2.14), which builds an irregular puncture (see appendix A.6 and for $N_{f}=2$ see [51]). We only study this limit for SQCD (see section 2.2.3), but the discussion applies to all our surface operators.

\subsection{SQED and Toda fundamental degenerate}

We review in this section the case of $\mathcal{N}=(2,2)$ SQED on $S^{2}$, namely a U(1) vector multiplet coupled to $N_{f}$ fundamental and $N_{f}$ antifundamental chiral multiplets, whose twisted masses (plus $R$-charges) we denote by $m_{s}$ and $\widetilde{m}_{s}$ for $1 \leq s \leq N_{f}$. It was shown [18] that the $S^{2}$ partition function of SQED matches an $A_{N_{f}-1}$ Toda CFT four-point function, up to a constant. We find that the constant factor reproduces the $S_{b}^{4}$ partition function of $N_{f}^{2}$ free hypermultiplets with masses (2.17), hence the Toda correlator in fact captures the partition function of the surface operator inserted in this free four dimensional theory. The precise relation is ${ }^{18}$

$$
Z_{S^{2} \subset S_{b}^{4}}^{\mathrm{SQED}}(m, \widetilde{m}, z, \bar{z})=A|x|^{2 \gamma_{0}}|1-x|^{2 \gamma_{1}}\left\langle\widehat{V}_{\alpha_{\infty}}(\infty) \widehat{V}_{\hat{m}}(1) \widehat{V}_{-b h_{1}}(x, \bar{x}) \widehat{V}_{\alpha_{0}}(0)\right\rangle .
$$

\footnotetext{
${ }^{18}$ As explained below (2.10), the factor $A|x|^{2 \gamma_{0}}|1-x|^{2 \gamma_{1}}$ can be absorbed into the partition function. To compare gauge theory and Toda CFT results it is best to keep the factor explicitly.
} 
The Toda CFT correlator (see appendix A.1 for conventions) features a degenerate field $\widehat{V}_{-b h_{1}}$ inserted at $x=(-1)^{N_{f}} z$ and labeled by the fundamental representation $\mathcal{R}\left(h_{1}\right)$ of $A_{N_{f}-1}$, a semi-degenerate field $\widehat{V}_{\hat{m}}$ at 1 , and two generic fields $\widehat{V}_{\alpha_{0}}$ and $\widehat{V}_{\alpha_{\infty}}$. Momenta are related to twisted masses through

$$
\begin{array}{rlrl}
\alpha_{0} & =Q-\frac{1}{b} \sum_{s=1}^{N_{f}} \mathrm{i} m_{s} h_{s}, & \hat{m} & =(\varkappa+b) h_{1}, \\
\alpha_{\infty}=Q-\frac{1}{b} \sum_{s=1}^{N_{f}} \mathrm{i} \widetilde{m}_{s} h_{s}, & \varkappa & =\frac{1}{b} \sum_{s=1}^{N_{f}}\left(1+\mathrm{i} m_{s}+\mathrm{i} \widetilde{m}_{s}\right),
\end{array}
$$

and the exponents and coefficient are

$$
\begin{aligned}
\gamma_{0} & =-\frac{1}{N_{f}} \sum_{s=1}^{N_{f}} \mathrm{i} m_{s}-\frac{N_{f}-1}{2}\left(b^{2}+1\right), \\
\gamma_{1} & =-\frac{N_{f}-1}{N_{f}} b^{2}+\frac{1}{N_{f}} \sum_{s=1}^{N_{f}}\left(\mathrm{i} m_{s}+\mathrm{i} \widetilde{m}_{s}\right), \\
A & =b^{N_{f}\left(1+b^{2}\right)-b^{2}-2 b \varkappa} .
\end{aligned}
$$

Permuting the $m_{s}$, or the $\widetilde{m}_{s}$, does not affect the partition function. This is reproduced in the Toda CFT by the invariance of $\widehat{V}_{\alpha_{0}}$ and $\widehat{V}_{\alpha_{\infty}}$ under Weyl transformations (the normalization is chosen to cancel reflection amplitudes). The similarity between $\alpha_{0}$ and $\alpha_{\infty}$ is also expected, as swapping them and changing $x$ to its inverse amounts in gauge theory to charge conjugation, which swaps $m_{s}$ and $\widetilde{m}_{s}$, and changes $z$ to its inverse. Under this transformation, $Z_{S^{2}}$ is invariant, while the Toda correlator receives a small shift controlled by the dimension $\Delta\left(-b h_{1}\right)$ of the degenerate insertion: this shift is absorbed by the factor $|x|^{2 \gamma_{0}}|1-x|^{2 \gamma_{1}}$.

In [18], the equality was shown directly thanks to known expressions [58] for the $W_{N_{f}}$ conformal block involved. The approach does not generalize, because conformal blocks with higher degenerate insertions were not previously known. ${ }^{19}$ Instead, we prove the correspondence for SQED (treated here) and SQCD (see section 2.2) by comparing monodromy matrices around branch points. In the main text, we find expansions around all branch points and compare leading terms, as this is enough to fix uniquely the dictionary between gauge theory and Toda CFT parameters. In appendix A.3 we derive the braiding matrices relating s-channel and u-channel expansions of the Toda CFT correlator, and their gauge theory analogues. The braiding matrices match. From this we deduce the matching of monodromy matrices around all branch points, expressed in a single basis, and not only of their eigenvalues compared in the main text. These results suffice to prove that the partition function and the correlator are equal.

To prepare for the somewhat technical computations ahead, we first go through the various steps here in the well-controlled case of SQED and Toda CFT fundamental degenerate fields. The expansions near $z=0$ and $z=\infty$ follow [18] closely, while the expansion near $z=(-1)^{N_{f}}$ is new. All three play an important role in later sections.

\footnotetext{
${ }^{19}$ We derive such explicit conformal blocks from our matchings in appendix A.5.
} 


\subsubsection{Expanding the SQED partition function}

The Coulomb branch expression for the partition function of SQED is

$$
Z_{S^{2}}^{\mathrm{SQED}}=\sum_{B \in \mathbb{Z}} \int_{\mathbb{R}} \frac{\mathrm{d} \sigma}{2 \pi} z^{\mathrm{i} \sigma+\frac{B}{2}} \bar{z}^{\mathrm{i} \sigma-\frac{B}{2}} \prod_{s=1}^{N_{f}}\left[\frac{\Gamma\left(-\mathrm{i} m_{s}-\mathrm{i} \sigma-\frac{B}{2}\right)}{\Gamma\left(1+\mathrm{i} m_{s}+\mathrm{i} \sigma-\frac{B}{2}\right)} \frac{\Gamma\left(-\mathrm{i} \widetilde{m}_{s}+\mathrm{i} \sigma+\frac{B}{2}\right)}{\Gamma\left(1+\mathrm{i} \widetilde{m}_{s}-\mathrm{i} \sigma+\frac{B}{2}\right)}\right]
$$

As we will see shortly, the contour of integration for $\sigma$ can be closed in the lower or upper half plane depending on whether $|z| \lessgtr 1$, leading to distinct expressions of $Z$ as a sum over poles lying in either half plane. We will match the resulting expressions with the s-channel and u-channel decompositions of the Toda CFT four-point correlator.

To find out which half-plane the contour should enclose, we study the asymptotic behavior of the integrand. First, rewrite the ratios of Gamma functions so that the numerator and denominator have no common poles,

$$
\frac{\Gamma\left(-v \pm \frac{B}{2}\right)}{\Gamma\left(1+v \pm \frac{B}{2}\right)}=(-1)^{\frac{B \mp|B|}{2}} \frac{\Gamma\left(-v+\frac{|B|}{2}\right)}{\Gamma\left(1+v+\frac{|B|}{2}\right)},
$$

and absorb the resulting sign $(-1)^{N_{f} B}$ by introducing

$$
x=(-1)^{N_{f}} z, \quad \bar{x}=(-1)^{N_{f}} \bar{z} .
$$

Thanks to $\frac{\Gamma(v+a)}{\Gamma(v+b)} \sim v^{a-b}$, valid when $|v| \rightarrow \infty$ away from the negative real axis, the integrand is

$$
\begin{gathered}
x^{\mathrm{i} \sigma+\frac{B}{2}} \bar{x}^{\mathrm{i} \sigma-\frac{B}{2}} \prod_{s=1}^{N_{f}}\left[\frac{\Gamma\left(-\mathrm{i} m_{s}-\mathrm{i} \sigma+\frac{|B|}{2}\right)}{\Gamma\left(1+\mathrm{i} m_{s}+\mathrm{i} \sigma+\frac{|B|}{2}\right)} \frac{\Gamma\left(-\mathrm{i} \widetilde{m}_{s}+\mathrm{i} \sigma+\frac{|B|}{2}\right)}{\Gamma\left(1+\mathrm{i} \widetilde{m}_{s}-\mathrm{i} \sigma+\frac{|B|}{2}\right)}\right] \\
\sim x^{\mathrm{i} \sigma+\frac{B}{2}} \bar{x}^{\mathrm{i} \sigma-\frac{B}{2}}\left(\sigma^{2}+\frac{B^{2}}{4}\right)^{-\sum_{s=1}^{N_{f}}\left(1+\mathrm{i} m_{s}+\mathrm{i} \widetilde{m}_{s}\right)}
\end{gathered}
$$

as $\left|\mathrm{i} \sigma \pm \frac{B}{2}\right| \rightarrow \infty$.

As long as we keep $\sigma \in \mathbb{R}$ on the integration contour, the factor $x^{\mathrm{i} \sigma+\frac{B}{2}} \bar{x}^{\mathrm{i} \sigma-\frac{B}{2}}$ is simply a phase. If $|x|=|z|<1$, this factor decays exponentially towards $\sigma \rightarrow-\mathrm{i} \infty$, hence the contour of integration can be closed in this half-plane. On the other hand, for $|x|=|z|>1$, the integrand decays exponentially in the $\sigma \rightarrow \mathrm{i} \infty$ half-plane.

The integrand (2.27) has poles whenever one of $-\mathrm{i} m_{p}-\mathrm{i} \sigma+\frac{|B|}{2}$ or $-\mathrm{i} \widetilde{m}_{p}+\mathrm{i} \sigma+\frac{|B|}{2}$ is a non-positive integer, that is, at

$$
\mathrm{i} \sigma=-\mathrm{i} m_{p}+k+\frac{|B|}{2} \quad \text { or } \quad \mathrm{i} \widetilde{m}_{p}-k-\frac{|B|}{2}
$$

for a fundamental or antifundamental flavour $1 \leq p \leq N_{f}$ and an integer $k \geq 0$. Since $R$-charges are positive, $-\mathrm{i} m_{p}$ has a positive real part and $\mathrm{i} \widetilde{m}_{p}$ a negative real part, hence the poles of the fundamental multiplets' one-loop determinants lie in the half-plane towards $\sigma \rightarrow-\mathrm{i} \infty$, while the other half plane contains those of antifundamental multiplets. 
Let us focus on the case $|x|=|z|<1$. We then sum residues of the integrand of (2.24) over poles (2.28) where $\mathrm{i} \sigma$ has a positive real part. This yields

$$
\begin{aligned}
Z=\sum_{p=1}^{N_{f}} & \sum_{k \geq 0} \sum_{B \in \mathbb{Z}}\left\{z^{-\mathrm{i} m_{p}+k+\frac{|B|+B}{2}} \bar{z}^{-\mathrm{i} m_{p}+k+\frac{|B|-B}{2}}\right. \\
& \left.\cdot \prod_{s=1}^{N_{f}}\left[\frac{\Gamma\left(-\mathrm{i} m_{s}+\mathrm{i} m_{p}-k-\frac{|B|+B}{2}\right)}{\Gamma\left(1+\mathrm{i} m_{s}-\mathrm{i} m_{p}+k+\frac{|B|-B}{2}\right)} \frac{\Gamma\left(-\mathrm{i} \widetilde{m}_{s}-\mathrm{i} m_{p}+k+\frac{|B|+B}{2}\right)}{\Gamma\left(1+\mathrm{i} \widetilde{m}_{s}+\mathrm{i} m_{p}-k-\frac{|B|-B}{2}\right)}\right]\right\},
\end{aligned}
$$

where the singular factor $\Gamma\left(-k-\frac{|B|+B}{2}\right)$ appearing for $s=p$ should be replaced by its residue $(-1)^{k+\frac{|B|+B}{2}} / \Gamma\left(1+k+\frac{|B|+B}{2}\right)$. Note that $k$ and $B$ appear as the combinations $k^{ \pm}=k+\frac{|B| \pm B}{2}$ only, and that the sums over $k \geq 0$ and $B \in \mathbb{Z}$ are equivalent to sums over $k^{+} \geq 0$ and $k^{-} \geq 0$. Hence,

$$
Z=\sum_{p=1}^{N_{f}} \sum_{k^{ \pm} \geq 0}(z \bar{z})^{-\mathrm{i} m_{p}} z^{k^{+}} \bar{z}^{k^{-}} \prod_{s=1}^{N_{f}}\left[\frac{\Gamma\left(-\mathrm{i} m_{s}+\mathrm{i} m_{p}-k^{+}\right)}{\Gamma\left(1+\mathrm{i} m_{s}-\mathrm{i} m_{p}+k^{-}\right)} \frac{\Gamma\left(-\mathrm{i} \widetilde{m}_{s}-\mathrm{i} m_{p}+k^{+}\right)}{\Gamma\left(1+\mathrm{i} \widetilde{m}_{s}+\mathrm{i} m_{p}-k^{-}\right)}\right],
$$

with the same caveat as above, namely, $\Gamma\left(-k^{+}\right) \rightarrow(-1)^{k^{+}} / \Gamma\left(1+k^{+}\right)$. Since each Gamma function argument depends on only one of $k^{+}$and $k^{-}$, the contribution from each flavour $p$ factorizes as the product of two series in (positive) powers of $z$ and of $\bar{z}$. We extract from the series a normalization factor (the value at $k^{ \pm}=0$ ), by writing the Gamma functions in terms of Pochhammer symbols $(a)_{n}=\frac{\Gamma(a+n)}{\Gamma(a)}$ and of $\gamma(x)=\frac{\Gamma(x)}{\Gamma(1-x)}$,

$$
\frac{\Gamma\left(-\mathrm{i} m_{s}+\mathrm{i} m_{p}-k^{+}\right)}{\Gamma\left(1+\mathrm{i} m_{s}-\mathrm{i} m_{p}+k^{-}\right)}=\frac{(-1)^{k^{+}} \gamma\left(-\mathrm{i} m_{s}+\mathrm{i} m_{p}\right)}{\left(1+\mathrm{i} m_{s}-\mathrm{i} m_{p}\right)_{k^{+}}\left(1+\mathrm{i} m_{s}-\mathrm{i} m_{p}\right)_{k^{-}}} .
$$

We deduce the partition function for $|x|=|z|<1$ in terms of "s-channel" vortex partition functions

$$
\begin{aligned}
Z & =\sum_{p=1}^{N_{f}}\left\{(x \bar{x})^{-\mathrm{i} m_{p}} \frac{\prod_{s \neq p}^{N_{f}} \gamma\left(-\mathrm{i} m_{s}+\mathrm{i} m_{p}\right)}{\prod_{s=1}^{N_{f}} \gamma\left(1+\mathrm{i} \widetilde{m}_{s}+\mathrm{i} m_{p}\right)} f_{p}^{(\mathrm{s})}(m, \widetilde{m}, x) f_{p}^{(\mathrm{s})}(m, \widetilde{m}, \bar{x})\right\}, \\
f_{p}^{(\mathrm{s})}(m, \widetilde{m}, x) & =\sum_{k \geq 0} x^{k} \prod_{s=1}^{N_{f}} \frac{\left(-\mathrm{i} \widetilde{m}_{s}-\mathrm{i} m_{p}\right)_{k}}{\left(1+\mathrm{i} m_{s}-\mathrm{i} m_{p}\right)_{k}}=F\left(\begin{array}{c}
\left(-\mathrm{i} \widetilde{m}_{s}-\mathrm{i} m_{p}\right), 1 \leq s \leq N_{f} \\
\left(1+\mathrm{i} m_{s}-\mathrm{i} m_{p}\right), s \neq p
\end{array} \mid x\right) .
\end{aligned}
$$

The $f_{p}^{(\mathrm{s})}$ are hypergeometric functions, related later on to s-channel conformal blocks in the Toda CFT. Similar computations for $|x|=|z|>1$ convert the sum over poles at $\mathrm{i} \sigma=\mathrm{i} \widetilde{m}_{p}-\cdots$ to a factorized form, related to the u-channel decomposition of a Toda CFT correlator,

$$
\begin{aligned}
Z & =\sum_{p=1}^{N_{f}}\left\{(x \bar{x})^{\mathrm{i} \widetilde{m}_{p}} \frac{\prod_{s \neq p}^{N_{f}} \gamma\left(-\mathrm{i} \widetilde{m}_{s}+\mathrm{i} \widetilde{m}_{p}\right)}{\prod_{s=1}^{N_{f}} \gamma\left(1+\mathrm{i} m_{s}+\mathrm{i} \widetilde{m}_{p}\right)} f_{p}^{(\mathrm{u})}(m, \widetilde{m}, x) f_{p}^{(\mathrm{u})}(m, \widetilde{m}, \bar{x})\right\}, \\
f_{p}^{(\mathrm{u})}(m, \widetilde{m}, x) & =\sum_{k \geq 0} x^{-k} \prod_{s=1}^{N_{f}} \frac{\left(-\mathrm{i} m_{s}-\mathrm{i} \widetilde{m}_{p}\right)_{k}}{\left(1+\mathrm{i} \widetilde{m}_{s}-\mathrm{i} \widetilde{m}_{p}\right)_{k}}=F\left(\begin{array}{c}
\left(-\mathrm{i} m_{s}-\mathrm{i} \widetilde{m}_{p}\right), 1 \leq s \leq N_{f} \\
\left(1+\mathrm{i} \widetilde{m}_{s}-\mathrm{i} \widetilde{m}_{p}\right), s \neq p
\end{array} \mid \frac{1}{x}\right) .
\end{aligned}
$$


The factorized results (2.32) and (2.34) reproduce the general form (2.9)

$$
Z=\sum_{\text {vacua }} \operatorname{res}\left[Z_{\mathrm{cl}}(\sigma, 0, z, \bar{z}) Z_{11}(m, \sigma, 0)\right] Z_{\mathrm{v}, p}(m, z) Z_{\overline{\mathrm{v}}, p}(m, \bar{z})
$$

obtained when localizing to the Higgs branch of the theory for positive and for negative FI parameter $\xi$, respectively. Indeed, Higgs branch vacua are labeled by solutions of

$$
\sum_{s=1}^{N_{f}}\left(\left|q_{s}\right|^{2}-\left|\widetilde{q}_{s}\right|^{2}\right)=\xi=-\frac{1}{2 \pi} \ln |z|
$$

and $\left(\sigma+m_{s}\right) q_{s}=0=\left(\sigma-\widetilde{m}_{s}\right) \widetilde{q}_{s}$ for all $s$. For $|z|<1$, that is, $\xi>0$, at least one of the positively charged fields $q_{s}$ is non-zero, thus $\sigma=-m_{s}$. For $|z|>1$, that is, $\xi<0$, one of the negatively charged fields is non-zero, and $\sigma=\widetilde{m}_{s}$. One easily checks that evaluating the classical contribution, and the residue of the one-loop contribution (which is the integrand of the Coulomb branch representation (2.24)) at those values of $\sigma$ and at $B=0$ yields the relevant factors in (2.32) and (2.34). The hypergeometric functions $f_{p}^{(\mathrm{s})}$ and $f_{p}^{(\mathrm{u})}$ obtained from factorization also match with known vortex and anti-vortex partition functions (see [17, 18]). For more general theories, factorization always yields explicit expressions for the vortex partition functions, while earlier methods soon become intractable.

The s-channel factors in (2.32) also have a Mellin-Barnes integral representation

$$
\begin{aligned}
& (-x)^{-\mathrm{i} m_{p}} f_{p}^{(\mathrm{s})}(x) \\
& \quad=\prod_{s=1}^{N_{f}}\left[\frac{\Gamma\left(1+\mathrm{i} m_{s}-\mathrm{i} m_{p}\right)}{\Gamma\left(-\mathrm{i} \widetilde{m}_{s}-\mathrm{i} m_{p}\right)}\right] \int_{-\mathrm{i} \infty}^{\mathrm{i} \infty} \frac{\mathrm{d} \kappa}{2 \pi \mathrm{i}} \frac{\prod_{s=1}^{N_{f}} \Gamma\left(-\mathrm{i} \widetilde{m}_{s}+\kappa\right)}{\prod_{s \neq p}^{N_{f}} \Gamma\left(1+\mathrm{i} m_{s}+\kappa\right)} \Gamma\left(-\kappa-\mathrm{i} m_{p}\right)(-x)^{\kappa}
\end{aligned}
$$

which converges for $|\arg (-x)|<\pi$, that is, away from the positive real axis. On the other hand, the s- and $\mathrm{u}$-channel expansions found above imply that the partition function has branch points at 0 and $\infty$, but is otherwise smooth away from the unit circle. Hence, the partition function can only have branch points at $x \in\{0,1, \infty\}$.

We have already given expansions near 0 and $\infty$, so we now focus on powers of $|1-x|^{2}$ as $x \rightarrow 1$. The Higgs branch localization has no analogue at $x=1$, because the FI parameter $\xi=-\frac{1}{2 \pi} \ln |z|$ vanishes and the manifold of solutions of $\sum_{s=1}^{N_{f}}\left(\left|q_{s}\right|^{2}-\left|\widetilde{q}_{s}\right|^{2}\right)=\xi$ experiences a flop transition. Instead, we find an explicit decomposition starting from the Coulomb branch integral.

As $x \rightarrow 1$, split the Coulomb branch representation (2.24) into the two regions, $\mid \mathrm{i} \sigma+$ $\left.\frac{B}{2}|\lessgtr| \ln x\right|^{-1}$. In the first, $x^{\mathrm{i} \sigma+\frac{B}{2}} \bar{x}^{\mathrm{i} \sigma-\frac{B}{2}}$ is given by a convergent series in integer powers of $\ln x$ and $\ln \bar{x}$ thanks to

$$
x^{\mathrm{i} \sigma+\frac{B}{2}}=\sum_{k \geq 0} \frac{\left(\mathrm{i} \sigma+\frac{B}{2}\right)^{k}}{k !}(\ln x)^{k} .
$$

In the second, the product of Gamma functions in the integrand can be approximated as (2.27) through Stirling's approximation, and the sum over $B$ can be replaced by a 
continuous integral, leading to a contribution

$$
\int \mathrm{d} B \frac{\mathrm{d} \sigma}{2 \pi} e^{\left(\mathrm{i} \sigma+\frac{B}{2}\right) \ln x} e^{\left(\mathrm{i} \sigma-\frac{B}{2}\right) \ln \bar{x}}\left(\sigma^{2}+\frac{B^{2}}{4}\right)^{-\Sigma}=\frac{1}{\pi} \int \mathrm{d} \rho \rho \mathrm{d} \theta e^{2 \mathrm{i} \rho \cos \theta|\ln x|} \rho^{-2 \Sigma},
$$

where $\Sigma=\sum_{s=1}^{N_{f}}\left(1+\mathrm{i} m_{s}+\mathrm{i} \widetilde{m}_{s}\right)$ and we applied the change of variables $\rho e^{i \theta}|\ln x|=$ $\left(\sigma-\mathrm{i} \frac{B}{2}\right) \ln x$. Rescaling then $\rho$ by $|\ln x|$, we find that the contribution behaves as

$$
|\ln x|^{2 \Sigma-2} \sim|1-x|^{2\left[-1+\sum_{s=1}^{N_{f}}\left(1+\mathrm{i} m_{s}+\mathrm{i} \widetilde{m}_{s}\right)\right]}
$$

as $x \rightarrow 1$, multiplied by a series in powers of $(1-x)$ and $(1-\bar{x})$. We thus find

$$
Z=|1-x|^{0} G(1-x, 1-\bar{x})+|1-x|^{2\left[-1+\sum_{s=1}^{N_{f}}\left(1+\mathrm{i} m_{s}+\mathrm{i} \widetilde{m}_{s}\right)\right.} H(1-x, 1-\bar{x})
$$

for some series $G$ and $H$ in positive integer powers of $1-x$ and $1-\bar{x}$. Since the $N_{f}$ terms of the Higgs branch expansions around $x=0$ and $\infty$ are linearly independent, the series $G$ and $H$ cannot both factorize. When studying the gauge theory analogue of the braiding matrix relating the s- and $\mathrm{u}$-channel expansions in appendix A.3, we find that $H$ factorizes as $h(1-x) \bar{h}(1-\bar{x})$, while $G$ is a sum of $N_{f}-1$ such factorized terms, with no preferred choice of splitting. We can expect the factorization of $H$ because in the limit $\left|\mathrm{i} \sigma \pm \frac{B}{2}\right| \rightarrow \infty$ the integrand (2.40) factorizes into functions of $\mathrm{i} \sigma \pm \frac{B}{2}$.

\subsubsection{Matching parameters for SQED}

We wish to equate the expansions of $Z$ obtained so far with an $A_{N_{f}-1}$ Toda CFT correlator. Since the $S^{2}$ partition function has branch points at $(-1)^{N_{f}} z \in\{0,1, \infty\}$, and factorizes when expanded around each of those points, the Toda correlator must be a four-point function with insertions at $0,1, \infty$, and $x=(-1)^{N_{f}} z$. The expansions near branch points have finitely many terms, hence the operator inserted at $x$ must be a degenerate operator $\widehat{V}_{-b \omega}$ (labeled by the highest weight $\omega$ of a representation $\mathcal{R}(\omega)$ of $A_{N_{f}-1}$ ), and the correlator has the form

$$
\left\langle\widehat{V}_{\alpha_{\infty}}(\infty) \widehat{V}_{\hat{m}}(1) \widehat{V}_{-b \omega}(x, \bar{x}) \widehat{V}_{\alpha_{0}}(0)\right\rangle
$$

The number of internal momenta allowed by the fusion rule for $\widehat{V}_{-b \omega}$ with a generic operator is equal to the dimension of $\mathcal{R}(\omega)$, hence $\mathcal{R}(\omega)$ must be the fundamental or antifundamental representation, to match the number of terms in (2.32) and (2.34). Without loss of generality (we can at this point conjugate all momenta), we choose the operator $\widehat{V}_{-b h_{1}}$, where $h_{1}$ is the highest weight of the fundamental representation. The momenta $\alpha_{0}, \hat{m}$ and $\alpha_{\infty}$ can then be obtained by comparing dimensions of Toda CFT operators with the powers of $|x|^{2}$ and of $|1-x|^{2}$ appearing in the expansions of $Z$ around $x=0, x=1$, and $x=\infty$. 
The s-channel decomposition of the Toda correlator is a sum over internal momenta $\alpha_{0}-b h_{p}$ labeling $W_{N_{f}}$ primary operators:

$$
\begin{aligned}
& \left\langle\widehat{V}_{\alpha_{\infty}}(\infty) \widehat{V}_{\hat{m}}(1) \widehat{V}_{-b h_{1}}(x, \bar{x}) \widehat{V}_{\alpha_{0}}(0)\right\rangle \\
& \quad=\sum_{p=1}^{N_{f}} \widehat{C}\left(\alpha_{\infty}, \hat{m}, \alpha_{0}-b h_{p}\right) \widehat{C}_{-b h_{1}, \alpha_{0}}^{\alpha_{0}-b h_{p}} \mathcal{F}_{\alpha_{0}-b h_{p}}^{(\mathrm{s})}\left[\begin{array}{cc}
\hat{m} & -b h_{1} \\
\alpha_{\infty} & \alpha_{0}
\end{array}\right](x) \mathcal{F}_{\alpha_{0}-b h_{p}}^{(\mathrm{s})}\left[\begin{array}{cc}
\hat{m} & -b h_{1} \\
\alpha_{\infty} & \alpha_{0}
\end{array}\right](\bar{x}),
\end{aligned}
$$

where $\widehat{C}$ denote three-point functions and $\mathcal{F}_{\alpha_{0}-b h_{p}}^{(\mathrm{s})}(x)$ are $W_{N_{f}}$ conformal blocks. Conformal invariance fixes $\mathcal{F}_{\alpha_{0}-b h_{p}}^{(\mathrm{s})}(x)=x^{\Delta\left(\alpha_{0}-b h_{p}\right)-\Delta\left(\alpha_{0}\right)-\Delta\left(-b h_{1}\right)}(1+\cdots)$, with a series $(1+\cdots)$ in positive integer powers of $x$. We compute

$$
\Delta\left(\alpha_{0}-b h_{p}\right)-\Delta\left(\alpha_{0}\right)-\Delta\left(-b h_{1}\right)=b\left\langle\alpha_{0}-Q, h_{p}\right\rangle+\frac{N_{f}-1}{2}\left(b^{2}+1\right) .
$$

This should be compared with the powers $x^{-\mathrm{i} m_{p}}$ appearing in (2.32). Since the weights $h_{p}$ sum to zero, $\sum_{p}\left\langle\alpha-Q, h_{p}\right\rangle=0$, and we must allow for an overall shift by $x^{\gamma_{0}}$ between the partition function and the correlator. Power matching then dictates

$$
b\left\langle\alpha_{0}-Q, h_{p}\right\rangle+\frac{N_{f}-1}{2}\left(b^{2}+1\right)+\gamma_{0}=-\mathrm{i} m_{p},
$$

up to permutations, from which we deduce $\alpha_{0}$ and $\gamma_{0}$ given in (2.20) and (2.21). Permuting the $m_{p}$ is equivalent to permuting the components of $\alpha_{0}-Q$, a Weyl reflection under which the primary operator $\widehat{V}_{\alpha_{0}}$ is invariant.

Next, the u-channel decomposition is a sum over the internal momenta $\alpha_{\infty}-b h_{p}$. Conformal invariance fixes $\mathcal{F}_{\alpha_{\infty}-b h_{p}}^{(\mathrm{u})}(x)=x^{\Delta\left(\alpha_{\infty}\right)-\Delta\left(\alpha_{\infty}-b h_{p}\right)-\Delta\left(-b h_{1}\right)}(1+\cdots)$, with a series $(1+\cdots)$ in negative integer powers of $x$. We compute

$$
\Delta\left(\alpha_{\infty}\right)-\Delta\left(\alpha_{\infty}-b h_{p}\right)-\Delta\left(-b h_{1}\right)=-b\left\langle\alpha_{\infty}-Q, h_{p}\right\rangle+\frac{N_{f}-1}{2}\left(b^{2}+1\right)+\frac{N_{f}-1}{N_{f}} b^{2},
$$

which should be compared with $x^{\mathrm{i} \widetilde{m}_{p}-\gamma_{0}}$. Once more, we must allow for an overall ambiguity: besides $x^{\gamma_{0}}$, the only other factor that can appear is $(1-x)^{\gamma_{1}}$, since the Toda correlator is only singular at 0,1 , and $\infty$. This factor does not alter powers at $x=0$, and the power matching at $x=\infty$ reads

$$
-b\left\langle\alpha_{\infty}-Q, h_{p}\right\rangle+\frac{N_{f}-1}{2}\left(b^{2}+1\right)+\frac{N_{f}-1}{N_{f}} b^{2}+\gamma_{0}+\gamma_{1}=\mathrm{i} \tilde{m}_{p}
$$

up to permutations: this fixes $\alpha_{\infty}$ and $\gamma_{1}$ to (2.20) and (2.22).

Third, the expansion of $Z$ near $x=1$ involves the leading powers $(1-x)^{0}$ with multiplicity $N_{f}-1$ and $(1-x)^{-1+\sum_{p=1}^{N_{f}}\left(1+\mathrm{i} m_{p}+\mathrm{i} \widetilde{m}_{p}\right)}$ with no multiplicity. On the Toda CFT side, the exponents that can appear in the t-channel are

$$
\begin{aligned}
& \Delta\left(\alpha_{1}-b h_{p}\right)-\Delta\left(\alpha_{1}\right)-\Delta\left(-b h_{1}\right)+\gamma_{1} \\
& \quad=b\left\langle\alpha_{1}-Q, h_{p}\right\rangle+\frac{N_{f}-1}{2}\left(b^{2}+1\right)-\frac{N_{f}-1}{N_{f}} b^{2}+\frac{1}{N_{f}} \sum_{p=1}^{N_{f}}\left(\mathrm{i} m_{p}+\mathrm{i} \widetilde{m}_{p}\right) .
\end{aligned}
$$


If $\alpha_{1}$ were generic, all shifts $-b h_{p}$ would be allowed by the fusion, but summing the powers (2.49) for $1 \leq p \leq N_{f}$ does not yield the similar gauge theory sum $-1+\sum_{p=1}^{N_{f}}(1+$ $\left.\mathrm{i} m_{p}+\mathrm{i} \widetilde{m}_{p}\right)$. Instead, we take $\alpha_{1}=\hat{m}=(\varkappa+b) h_{1}$ to be a semi-degenerate momentum (with a shift by $b$ to simplify expressions), so that the fusion rule only allows shifts to $\hat{m}-b h_{2}$ and $\hat{m}-b h_{1}$. Setting the exponent for a shift $\hat{m}-b h_{2}$ to 0 fixes $\varkappa$ to (2.20), and the second power matches (setting $\hat{m}-b h_{1}$ to 0 instead would fail to match the second power). The $\mathrm{SU}\left(N_{f}\right) \times \mathrm{SU}\left(N_{f}\right) \times \mathrm{U}(1)$ flavour symmetry of the gauge theory is reproduced by the two generic and one semi-degenerate operators in the correlator, allowing us to package the twisted masses of fundamental chiral multiplets into $\alpha_{0}$, those of antifundamental multiplets into $\alpha_{\infty}$, and the axial mass into $\hat{m}$.

Finally, the overall constant $A$ is fixed in appendix A.2 by comparing gauge theory one-loop determinants and Toda three-point functions: for $A$ given by (2.23),

$$
Z_{S_{b}^{4}}^{\mathrm{free}} \frac{\prod_{s \neq p}^{N_{f}} \gamma\left(\mathrm{i} m_{p}-\mathrm{i} m_{s}\right)}{\prod_{t=1}^{N_{f}} \gamma\left(1+\mathrm{i} m_{p}+\mathrm{i} \widetilde{m}_{t}\right)}=A \widehat{C}\left(\alpha_{\infty},(\varkappa+b) h_{1}, \alpha_{0}-b h_{p}\right) \widehat{C}_{-b h_{1}, \alpha_{0}}^{\alpha_{0}-b h_{p}} .
$$

The same relation holds for u-channel constant factors (with an identical value of $A$ ), as we can obtain most readily thanks to the invariance of $Z$ under $m_{p} \leftrightarrow \widetilde{m}_{p}$ and $z \leftrightarrow \frac{1}{z}$ (gauge theory charge conjugation) and equivalently of the Toda correlator (up to a shift in exponents) under $\alpha_{0} \leftrightarrow \alpha_{\infty}$ and $x \leftrightarrow \frac{1}{x}$.

We have thus fixed how gauge theory and Toda CFT parameters match. One way to prove the matching is to directly equate gauge theory factors with conformal blocks as done in [18], but this approach does not generalize. Instead, we show in appendix A.3 that the matrix to change basis from s-channel factors $x^{-\mathrm{i} m_{p}} f_{p}^{(\mathrm{s})}(x)$ to $\mathrm{u}$-channel factors is identical to the appropriate braiding matrix in the Toda CFT. Since the eigenvalues of monodromies around 0 and $\infty$ also match up to shifts by the $\gamma_{i}$ as we just saw, the monodromy matrices themselves agree. The last monodromy matrix, around $x=1$, thus also matches. Therefore, the partition function and the correlator differ by a factor with no monodromy. Since the precise exponents match, the relative factor is in fact constant, and comparing constant coefficients establishes the matching (2.19).

\subsection{SQCD and Toda antisymmetric degenerate}

We now extend the matching to the case of $\mathcal{N}=(2,2)$ SQCD, that is, a $\mathrm{U}(N)$ vector multiplet coupled to $N_{f}$ fundamental and $N_{f}$ antifundamental chiral multiplets, with twisted masses (plus $R$-charges) $m_{s}$ and $\widetilde{m}_{s}$. The partition function of the $S^{2}$ surface operator defined by this theory coupled to $N_{f}^{2}$ hypermultiplets with masses $(2.17)$ on $S_{b}^{4}$ is captured by a Toda CFT four-point function with a degenerate operator $\widehat{V}_{-b \omega_{N}}$ labeled by the $N$-th antisymmetric representation of $A_{N_{f}-1}$. Explicitly, we prove that ${ }^{20}$

$$
Z_{S^{2} \subset S_{b}^{4}}^{\mathrm{U}(N) \operatorname{SQCD}}(m, \widetilde{m}, z, \bar{z})=A|x|^{2 \gamma_{0}}|1-x|^{2 \gamma_{1}}\left\langle\widehat{V}_{\alpha_{\infty}}(\infty) \widehat{V}_{\hat{m}}(1) \widehat{V}_{-b \omega_{N}}(x, \bar{x}) \widehat{V}_{\alpha_{0}}(0)\right\rangle
$$

\footnotetext{
${ }^{20}$ As explained below (2.10), the factor $A|x|^{2 \gamma_{0}}|1-x|^{2 \gamma_{1}}$ can be absorbed into the partition function. To compare gauge theory and Toda CFT results it is best to keep the factor explicitly.
} 
with $x=(-1)^{N_{f}+N-1} z$, momenta

$$
\begin{aligned}
\alpha_{0}=Q-\frac{1}{b} \sum_{s=1}^{N_{f}} \mathrm{i} m_{s} h_{s}, & \hat{m}=(\varkappa+N b) h_{1}, \\
\alpha_{\infty}=Q-\frac{1}{b} \sum_{s=1}^{N_{f}} \mathrm{i} \widetilde{m}_{s} h_{s}, & \varkappa=\frac{1}{b} \sum_{s=1}^{N_{f}}\left(1+\mathrm{i} m_{s}+\mathrm{i} \widetilde{m}_{s}\right),
\end{aligned}
$$

and coefficients

$$
\begin{aligned}
\gamma_{0} & =-\frac{N}{N_{f}} \sum_{s=1}^{N_{f}} \mathrm{i} m_{s}-\frac{N\left(N_{f}-N\right)}{2}\left(b^{2}+1\right), \\
\gamma_{1} & =-\frac{N\left(N_{f}-N\right)}{N_{f}} b^{2}+\frac{N}{N_{f}} \sum_{s=1}^{N_{f}}\left(\mathrm{i} m_{s}+\mathrm{i} \widetilde{m}_{s}\right), \\
A & =b^{N N_{f}\left(1+b^{2}\right)-N^{2} b^{2}-2 N b \varkappa} .
\end{aligned}
$$

Setting $N=1$ in (2.51) reproduces the SQED matching (2.19). We recognize the same symmetries as SQED. Permuting twisted masses $m_{s}$ or $\widetilde{m}_{s}$ amounts to a Weyl transformation of $\alpha_{0}$ or $\alpha_{\infty}$. Gauge theory charge conjugation, which swaps $m_{s} \leftrightarrow \widetilde{m}_{s}$ and $z \leftrightarrow \frac{1}{z}$, corresponds to the conformal map $(\infty, 1, x, 0) \rightarrow\left(0,1, \frac{1}{x}, \infty\right)$, which exchanges $\alpha_{0} \leftrightarrow \alpha_{\infty}$ and $x \leftrightarrow \frac{1}{x}$ in the Toda CFT correlator.

We start the analysis from the Coulomb branch representation

$$
\begin{aligned}
& Z_{S^{2}}^{\mathrm{SQCD}}=\frac{1}{N !} \sum_{B \in \mathbb{Z}^{N}} \int_{\mathbb{R}^{N}} \frac{\mathrm{d}^{N} \sigma}{(2 \pi)^{N}}\left\{\left[(-1)^{N-1} z\right]^{\operatorname{Tr}\left(\mathrm{i} \sigma+\frac{B}{2}\right)}\left[(-1)^{N-1} \bar{z}\right]^{\operatorname{Tr}\left(\mathrm{i} \sigma-\frac{B}{2}\right)}\right. \\
& \left.\cdot \prod_{i<j}\left[\left(\sigma_{i}-\sigma_{j}\right)^{2}+\frac{\left(B_{i}-B_{j}\right)^{2}}{4}\right] \prod_{j=1}^{N} \prod_{s=1}^{N_{f}}\left[\frac{\Gamma\left(-\mathrm{i} m_{s}-\mathrm{i} \sigma_{j}-\frac{B_{j}}{2}\right)}{\Gamma\left(1+\mathrm{i} m_{s}+\mathrm{i} \sigma_{j}-\frac{B_{j}}{2}\right)} \frac{\Gamma\left(-\mathrm{i} \widetilde{m}_{s}+\mathrm{i} \sigma_{j}+\frac{B_{j}}{2}\right)}{\Gamma\left(1+\mathrm{i} \widetilde{m}_{s}-\mathrm{i} \sigma_{j}+\frac{B_{j}}{2}\right)}\right]\right\} .
\end{aligned}
$$

The partition function can be studied in the same way as that of SQED, by closing the integration contours towards either half-plane depending on whether $|z| \lessgtr 1$, thus obtaining an s-channel and a u-channel decompositions akin to (2.32) and (2.34). Interestingly, there is a shortcut, as the SQCD partition function can be expressed as a differential operator acting on the product of $N$ copies of the SQED partition function:

$$
Z_{S^{2}}^{\mathrm{SQCD}}=\frac{1}{N !}\left[\prod_{i<j}\left[-\left(z_{i} \partial_{z_{i}}-z_{j} \partial_{z_{j}}\right)\left(\bar{z}_{i} \partial_{\bar{z}_{i}}-\bar{z}_{j} \partial_{\bar{z}_{j}}\right)\right] \prod_{j=1}^{N} Z_{S^{2}}^{\mathrm{SQED}}\left(m, \widetilde{m}, z_{j}, \bar{z}_{j}\right)\right]_{\substack{z_{j}=(-1)^{N-1} z \\ \bar{z}_{j}=(-1)^{N-1} \bar{z}}}
$$

Since the differential operator cannot introduce branch points, the SQCD partition function has the same branch points $(-1)^{N-1} z \in\left\{0,(-1)^{N_{f}}, \infty\right\}$ as the SQED partition function, and we switch to using the coordinate $x=(-1)^{N_{f}+N-1} z$. 


\subsubsection{Expanding the SQCD partition function}

Using the s-channel decomposition (2.32) of $Z^{\mathrm{SQED}}$ in the above yields a sum over flavours $1 \leq p_{1}, \ldots, p_{N} \leq N_{f}$. The summand factorizes, since both the differential operator and the terms in $Z^{\mathrm{SQED}}$ are products of a holomorphic and an antiholomorphic parts. The holomorphic and the antiholomorphic factors are each totally antisymmetric in the $p_{j}$, hence reducing the sum to $1 \leq p_{1}<\cdots<p_{N} \leq N_{f}$. Explicitly,

$$
Z=\sum_{1 \leq p_{1}<\cdots<p_{N} \leq N_{f}}\left[(x \bar{x})^{-\sum_{j=1}^{N} \mathrm{i} m_{p_{j}}} \prod_{j=1}^{N} \frac{\prod_{s \notin\{p\}}^{N_{f}} \gamma\left(-\mathrm{i} m_{s}+\mathrm{i} m_{p_{j}}\right)}{\prod_{s=1}^{N_{f}} \gamma\left(1+\mathrm{i} \widetilde{m}_{s}+\mathrm{i} m_{p_{j}}\right)} f_{\{p\}}^{(\mathrm{s})}(x) f_{\{p\}}^{(\mathrm{s})}(\bar{x})\right]
$$

where we have canceled $\prod_{i \neq j} \gamma\left(-\mathrm{i} m_{p_{i}}+\mathrm{i} m_{p_{j}}\right)=\prod_{i \neq j}\left(\mathrm{i} m_{p_{i}}-\mathrm{i} m_{p_{j}}\right)^{-1}$ and defined

$$
\begin{aligned}
f_{\{p\}}^{(\mathrm{s})}(x) & =\left[\prod_{i<j} \frac{-\mathrm{i} m_{p_{i}}+\mathrm{i} m_{p_{j}}+x_{i} \partial_{x_{i}}-x_{j} \partial_{x_{j}}}{-\mathrm{i} m_{p_{i}}+\mathrm{i} m_{p_{j}}} \prod_{j=1}^{N} f_{p_{j}}^{(\mathrm{s})}\left(x_{j}\right)\right]_{x_{j}=x} \\
& =\sum_{k_{1}, \ldots, k_{N} \geq 0} \frac{x^{\sum_{j=1}^{N} k_{j}}}{\prod_{j=1}^{N} k_{j} !} \frac{\prod_{j=1}^{N} \prod_{s=1}^{N_{f}}\left(-\mathrm{i} \widetilde{m}_{s}-\mathrm{i} m_{p_{j}}\right)_{k_{j}}}{\prod_{i \neq j}^{N}\left(\mathrm{i} m_{p_{i}}-\mathrm{i} m_{p_{j}}-k_{i}\right)_{k_{j}} \prod_{j=1}^{N} \prod_{s \notin\{p\}}^{N_{f}}\left(1+\mathrm{i} m_{s}-\mathrm{i} m_{p_{j}}\right)_{k_{j}}},
\end{aligned}
$$

a series in positive integer powers of $x$, with radius of convergence 1 , and whose first term is normalized to be 1 . Similarly, the $\mathrm{u}$-channel expansion near $x=\infty$ reads

$$
Z=\sum_{1 \leq p_{1}<\cdots<p_{N} \leq N_{f}}\left[(x \bar{x})^{\sum_{j=1}^{N} \mathrm{i} \widetilde{m}_{p_{j}}} \frac{\prod_{j=1}^{N} \prod_{s \notin\{p\}}^{N_{f}} \gamma\left(-\mathrm{i} \widetilde{m}_{s}+\mathrm{i} \widetilde{m}_{p_{j}}\right)}{\prod_{j=1}^{N} \prod_{s=1}^{N_{f}} \gamma\left(1+\mathrm{i} m_{s}+\mathrm{i} \widetilde{m}_{p_{j}}\right)} f_{\{p\}}^{(\mathrm{u})}(x) f_{\{p\}}^{(\mathrm{u})}(\bar{x})\right]
$$

where

$$
f_{\{p\}}^{(\mathrm{u})}(x)=\sum_{k_{1}, \ldots, k_{N} \geq 0} \frac{x^{-\sum_{j=1}^{N} k_{j}}}{\prod_{j=1}^{N} k_{j} !} \frac{\prod_{j=1}^{N} \prod_{s=1}^{N_{f}}\left(-\mathrm{i} m_{s}-\mathrm{i} \widetilde{m}_{p_{j}}\right)_{k_{j}}}{\prod_{i \neq j}^{N}\left(\mathrm{i} \widetilde{m}_{p_{i}}-\mathrm{i} \widetilde{m}_{p_{j}}-k_{i}\right)_{k_{j}} \prod_{j=1}^{N} \prod_{s \notin\{p\}}^{N_{f}}\left(1+\mathrm{i} \widetilde{m}_{s}-\mathrm{i} \widetilde{m}_{p_{j}}\right)_{k_{j}}}
$$

are series in negative integer powers of $x$.

The s- and u-channel decompositions above can also be obtained by localizing to the Higgs branch of the theory, with a positive or a negative FI parameter. In this setting, they arise as sums over Higgs branch vacua, labeled by solutions $\left(\sigma, q_{s}, \widetilde{q}_{s}\right)$ of

$$
\begin{aligned}
\left(\sigma+m_{s}\right) q_{s} & =0 \\
\left(-\sigma+\widetilde{m}_{s}\right) \widetilde{q}_{s} & =0
\end{aligned} \quad \sum_{s=1}^{N_{f}}\left(q_{s} q_{s}^{\dagger}-\widetilde{q}_{s}^{\dagger} \widetilde{q}_{s}\right)=\xi \operatorname{id}_{N},
$$

up to gauge transformations. In the region $|x|=|z|<1$, that is, $\xi>0$, the D-term equation (2.63) can be rewritten as

$$
\sum_{s=1}^{N_{f}} q_{s} q_{s}^{\dagger}=\xi \operatorname{id}_{N}+\sum_{s=1}^{N_{f}} \widetilde{q}_{s}^{\dagger} \widetilde{q}_{s}
$$


which is positive definite, hence has full rank $N$. Therefore, the non-zero vectors $q_{s}$, which are eigenvectors of $\sigma$, span $\mathbb{C}^{N_{f}}$. The eigenvalues of $\sigma$ are thus completely fixed to be $-m_{p_{j}}$ for a choice of $N$ distinct flavours $p_{j}$. On the contrary, for $|x|=|z|>1$, that is, $\xi<0$, the antifundamental chiral fields $\widetilde{q}_{s}$ span $\mathbb{C}^{N_{f}}$, and $\sigma$ has eigenvalues $\widetilde{m}_{p_{j}}$. The classical and one-loop contributions derived for each of those vacua is equal to those appearing in (2.58) and (2.61). More tediously, one checks that the vortex partition functions are indeed given by $f_{\{p\}}^{(\mathrm{s})}(x)$ and $f_{\{p\}}^{(\mathrm{u})}(x)$.

Once more, the t-channel is the most troublesome. We know from (2.42) the expansion of the SQED partition function near $x=1$, leading to

$$
Z^{\mathrm{SQED}}=G(1-x, 1-\bar{x})+|1-x|^{2(\gamma-1)} h(1-x) \bar{h}(1-\bar{x}),
$$

where

$$
\gamma=\sum_{s=1}^{N_{f}}\left(1+\mathrm{i} m_{s}+\mathrm{i} \widetilde{m}_{s}\right)
$$

The functions $G$ and $h \bar{h}$ are series in positive integer powers of $1-x$ and $1-\bar{x}$, and $G$ does not factorize because the eigenvalue 1 of the monodromy has multiplicity $N_{f}-1$. Plug this t-channel expansion into (2.57):

$$
\begin{aligned}
Z^{\mathrm{SQCD}}(z, \bar{z})=\frac{1}{N !}\left[\prod_{i<j}[\right. & \left.-\left(x_{i} \partial_{x_{i}}-x_{j} \partial_{x_{j}}\right)\left(\bar{x}_{i} \partial_{\bar{x}_{i}}-\bar{x}_{j} \partial_{\bar{x}_{j}}\right)\right] \\
& \left.\cdot \prod_{j=1}^{N}\left\{G\left(1-x_{j}, 1-\bar{x}_{j}\right)+\left|1-x_{j}\right|^{2 \gamma-2} h\left(1-x_{j}\right) \bar{h}\left(1-\bar{x}_{j}\right)\right\}\right]_{\substack{x_{j}=x \\
\bar{x}_{j}=\bar{x}}} .
\end{aligned}
$$

Among the $2^{N}$ terms in the product of SQED partition functions, any which contains the factor $\left|1-x_{j}\right|^{2 \gamma-2} h\left(1-x_{j}\right) \bar{h}\left(1-\bar{x}_{j}\right)$ for two indices $i$ and $j$ is annihilated by $x_{i} \partial_{x_{i}}-x_{j} \partial_{x_{j}}$, hence does not contribute. The annihilation does not take place when $G\left(1-x_{j}, 1-\bar{x}_{j}\right)$ appears twice, as it relies on separating the holomorphic and antiholomorphic parts. Thus, $1+N$ terms remain, and we can replace the product by

$$
\prod_{j=1}^{N} G\left(1-x_{j}, 1-\bar{x}_{j}\right)+\sum_{j=1}^{N}\left|1-x_{j}\right|^{2 \gamma-2} h\left(1-x_{j}\right) \bar{h}\left(1-\bar{x}_{j}\right) \prod_{i \neq j}^{N} G\left(1-x_{i}, 1-\bar{x}_{i}\right) .
$$

Derivatives acting on $G, h$ and $\bar{h}$ yield other series in positive integer powers of $1-x_{j}$ and $1-\bar{x}_{j}$, hence for the purpose of finding exponents for $|1-x|^{2}$ we only need to keep track of $\left|1-x_{j}\right|^{2 \gamma-2}$. At most $(N-1) x_{j}$ derivatives can affect it, hence the SQCD partition function takes the form

$$
Z^{\mathrm{SQCD}}(z, \bar{z})=G^{\prime}(1-x, 1-\bar{x})+|1-x|^{2(\gamma-N)} H^{\prime}(1-x, 1-\bar{x}),
$$

for some series $G^{\prime}$ and $H^{\prime}$. The two terms correspond to eigenvalues 1 and $e^{2 \pi \mathrm{i}(\gamma-N)}$ of the monodromy around $x=1$. We find out the multiplicities with which the powers appear by doing a finer expansion: split $G\left(1-x_{j}, 1-\bar{x}_{j}\right)=\sum_{i=1}^{N_{f}-1} g_{i}\left(1-x_{j}\right) \bar{g}_{i}\left(1-\bar{x}_{j}\right)$ 
non-canonically. Antisymmetry restricts the sum of $N_{f}^{N}$ terms to $\left(\begin{array}{c}N_{f} \\ N\end{array}\right)$, each of which is a product of $N$ distinct terms of $Z^{\mathrm{SQED}}$ among $h \bar{h}$ and the $g_{i} \bar{g}_{i}$. The exponent for a given combination is $2(\gamma-N)$ if $h \bar{h}$ appears, and 0 otherwise. The multiplicity of $|1-x|^{0}$ is thus $\left(\begin{array}{c}N_{f}-1 \\ N\end{array}\right)$, and that of $|1-x|^{2(\gamma-N)}$ is $\left(\begin{array}{c}N_{f}-1 \\ N-1\end{array}\right)$.

\subsubsection{Matching parameters for SQCD}

We are at last ready to match SQCD and Toda CFT parameters. The partition function depends on a single parameter $x$ encoded as the position of a puncture, hence we expect a four-point function on the Toda side. The s-channel and u-channel decompositions involve $\left(\begin{array}{c}N_{f} \\ N\end{array}\right)$ terms, hence the Toda degenerate operator is labeled by the $N$-th antisymmetric representation $\mathcal{R}\left(\omega_{N}\right)$ of $A_{N_{f}-1}$, which has the correct dimension. The highest weight of this representation is $\omega_{N}=h_{1}+\cdots+h_{N}$, and its weights are $h_{\{p\}}=h_{p_{1}}+\cdots+h_{p_{N}}$, labeled by $N$-element sets $1 \leq p_{1}<\cdots<p_{N} \leq N_{f}$.

The s-channel Toda exponents

$$
\begin{aligned}
\Delta\left(\alpha_{0}-b h_{\{p\}}\right) & -\Delta\left(\alpha_{0}\right)-\Delta\left(-b \omega_{N}\right)+\gamma_{0} \\
= & b \sum_{j=1}^{N}\left\langle\alpha_{0}-Q, h_{p_{j}}\right\rangle+\frac{N\left(N_{f}-N\right)}{2}\left(b^{2}+1\right)+\gamma_{0}
\end{aligned}
$$

must be equal to $-\sum_{j=1}^{N} \mathrm{i} m_{p_{j}}$ from gauge theory (up to permutations): this constraint fixes $\alpha_{0}$ and $\gamma_{0}$ as given in (2.52) and (2.53). Matching powers in the u-channel,

$$
\begin{aligned}
\sum_{j=1}^{N} \mathrm{i} \widetilde{m}_{p_{j}} & =\Delta\left(\alpha_{\infty}\right)-\Delta\left(\alpha_{\infty}-b h_{\{p\}}\right)-\Delta\left(-b \omega_{N}\right)+\gamma_{0}+\gamma_{1} \\
& =-b \sum_{j=1}^{N}\left\langle\alpha_{\infty}-Q, h_{p_{j}}\right\rangle+\frac{N\left(N_{f}-N\right)}{2}\left(b^{2}+1\right)+\frac{N\left(N_{f}-N\right)}{N_{f}} b^{2}+\gamma_{0}+\gamma_{1}
\end{aligned}
$$

fixes $\alpha_{\infty}$ and $\gamma_{1}$.

We finally match powers in the t-channel. From our SQED experience, we expect the momentum at 1 to be the semi-degenerate $\hat{m}=(\varkappa+N b) h_{1}$ (the shift by $N b$ simplifies expressions). We compute the exponents

$$
\begin{array}{r}
\Delta\left((\varkappa+N b) h_{1}-b h_{\{p\}}\right)-\Delta\left((\varkappa+N b) h_{1}\right)-\Delta\left(-b \omega_{N}\right)+\gamma_{1} \\
=\left(b \varkappa+N b^{2}\right)\left\langle h_{1}, h_{\{p\}}\right\rangle+\frac{N}{N_{f}}\left[\sum_{s=1}^{N_{f}}\left(1+\mathrm{i} m_{s}+\mathrm{i} \widetilde{m}_{s}\right)+N b^{2}\right] \\
+\left(1+b^{2}\right) \sum_{j=1}^{N}\left(p_{j}-j-1\right),
\end{array}
$$

where $\left\langle h_{1}, h_{\{p\}}\right\rangle=\delta_{1 \in\{p\}}-N / N_{f}$. Two different sets $\{p\}$ must reproduce the gauge theory exponents 0 and $-N+\sum_{s=1}^{N_{f}}\left(1+\mathrm{i} m_{s}+\mathrm{i} \widetilde{m}_{s}\right)$. One set must contain 1 and the other not, since the exponents would otherwise only differ by an integer multiple of $1+b^{2}$ : this fixes 
$\varkappa= \pm \sum_{s=1}^{N_{f}}\left(1+\mathrm{i} m_{s}+\mathrm{i} \widetilde{m}_{s}\right)+n\left(b+\frac{1}{b}\right)$ for some integer $n$. Comparing the coefficients of $\sum_{s=1}^{N_{f}}\left(1+\mathrm{i} m_{s}+\mathrm{i} \widetilde{m}_{s}\right)$ selects the positive sign, and also implies that the exponent 0 corresponds to a case where $1 \notin\{p\}$ while the other exponent has $1 \in\left\{p^{\prime}\right\}$. Comparing the coefficients of $b^{2}+1$, the Toda CFT and gauge exponents match if

$$
-\frac{N}{N_{f}} n+\sum_{j=1}\left(p_{j}-j-1\right)=0 \quad \text { and } \quad \frac{N_{f}-N}{N_{f}} n+\sum_{j=1}\left(p_{j}^{\prime}-j-1\right)=-N
$$

for the choices of $\{p\}$ and $\left\{p^{\prime}\right\}$ corresponding to the two exponents. Since $1 \notin\{p\}, p_{j} \geq j+1$ and the first relation implies $n \leq 0$. Since $p_{j}^{\prime} \geq j$, the second implies $n \geq 0$, and we conclude that $\varkappa$ is given by $(2.52)$, that $\{p\}=\llbracket 2, N+1 \rrbracket$ and that $\left\{p^{\prime}\right\}=\llbracket 1, N \rrbracket .{ }^{21}$ After we show independently that the partition function and Toda correlator are equal, we deduce that the fusion of $\widehat{V}_{-b \omega_{N}}$ with $\widehat{V}_{\varkappa^{\prime} h_{1}}$ allows the momenta $\varkappa^{\prime} h_{1}-b \omega_{N}$ and $\varkappa^{\prime} h_{1}+b h_{1}-b \omega_{N+1}$. This is consistent with the case $\varkappa^{\prime}=-k b$ for which the semi-degenerate insertion becomes a degenerate field labeled by the $k$-th antisymmetric representation: the tensor product of this representation with the $N$-th antisymmetric splits as a sum of two irreducible representations of $A_{N_{f}-1}$, with highest weights $k h_{1}+\omega_{N}$ and $(k-1) h_{1}+\omega_{N+1}$. We discuss such fusion rules further in appendix A.4.

Last, we fix the constant $A$. We check in appendix A.2 that the one-loop determinant and the three-point functions appearing in the s-channel decompositions of $Z^{\mathrm{SQCD}}$ and of the Toda correlator match, for $A$ given in (2.55):

$$
Z_{S_{b}^{4}}^{\mathrm{free}} \frac{\prod_{s \notin\{p\}}^{N_{f}} \prod_{t \in\{p\}} \gamma\left(\mathrm{i} m_{t}-\mathrm{i} m_{s}\right)}{\prod_{s=1}^{N_{f}} \prod_{t \in\{p\}} \gamma\left(1+\mathrm{i} m_{t}+\mathrm{i} \widetilde{m}_{s}\right)}=A \widehat{C}\left(\alpha_{\infty},(\varkappa+N b) h_{1}, \alpha_{0}-b h_{\{p\}}\right) \widehat{C}_{-b \omega_{N}, \alpha_{0}}^{\alpha_{0}-b h_{\{p\}}} .
$$

Having settled the dictionary above, we know that gauge theory and Toda CFT monodromy matrices around each of 0,1 and $\infty$ have matching eigenvalues. In appendix A.3, we compute the braiding matrix of $\widehat{V}_{-b \omega_{N}}$ and $\widehat{V}_{\hat{m}}$ by combining the fusion of $N$ operators $\widehat{V}_{-b h_{1}}$ into $\widehat{V}_{-b \omega_{N}}$ with the braiding matrices for each individual $\widehat{V}_{-b h_{1}}$ with $\widehat{V}_{\hat{m}}$. The result agrees with the analogue for SQCD, an antisymmetric combination of the matrix for SQED, worked out in the same appendix. Therefore, the monodromy matrices around 0 and around $\infty$ are equal for SQCD and the Toda CFT. Monodromy matrices around 1 then also match, hence the Toda CFT correlator and gauge theory partition function are equal up to a factor with no monodromy, which is constant since the precise exponents at 0,1 and $\infty$ match. The constant factors work out, thereby concluding the proof of the matching (2.51).

\subsubsection{Decoupled multiplets and irregular puncture}

In this section, we give large twisted masses to $N_{f}-\widetilde{N_{f}}$ of the $N_{f}$ antifundamental chiral multiplets of the SQCD surface operator, hence to $N_{f}\left(N_{f}-\widetilde{N_{f}}\right)$ of the four dimensional hypermultiplets. The massive multiplets decouple, and we obtain in this limit (2.76) a surface operator described by a $\mathrm{U}(N)$ vector multiplet, $N_{f}$ fundamental and $\widetilde{N_{f}}<N_{f}$

\footnotetext{
${ }^{21}$ Here and later we denote integer intervals by $\llbracket a, b \rrbracket=[a, b] \cap \mathbb{Z}$.
} 
antifundamental chiral multiplets, coupled to the remaining $N_{f} \widetilde{N_{f}}$ free hypermultiplets. On the Toda CFT side of the matching (2.51), the limit amounts to building a Toda CFT irregular puncture from the collision of two vertex operators. We give the precise matching (2.83) in the case $\widetilde{N_{f}}=N_{f}-1$, and claim that further limits for $\widetilde{N}_{f} \leq N_{f}-2$ also lead to well-defined irregular punctures.

In a two dimensional $\mathcal{N}=(2,2)$ gauge theory, whenever the total charge $Q=\sum_{i} Q_{i}$ of all chiral multiplets under a given $\mathrm{U}(1)$ gauge group factor is non-zero (in our case, $Q=$ $N_{f}-\widetilde{N}_{f}>0$ ), the corresponding FI parameter runs logarithmically, and the theta angle is shifted. An ultraviolet cutoff can be introduced supersymmetrically by enriching the theory with a single "spectator" chiral multiplet of large twisted mass ${ }^{22} \Lambda \in \mathbb{R}$ and $\mathrm{U}(1)$ charge $-Q$, or with $Q$ antifundamental spectator chiral multiplets of twisted masses $\Lambda$. We take the latter approach, as the resulting enriched theory is simply SQCD with $N_{f}$ fundamental and $N_{f}$ antifundamental chiral multiplets. Each spectator chiral multiplet brings a one-loop contribution

$$
\prod_{j=1}^{N} \frac{\Gamma\left(-\mathrm{i} \Lambda+\mathrm{i} \sigma_{j}+\frac{B_{j}}{2}\right)}{\Gamma\left(1+\mathrm{i} \Lambda-\mathrm{i} \sigma_{j}+\frac{B_{j}}{2}\right)} \stackrel{\Lambda \rightarrow \infty}{\sim} \prod_{j=1}^{N}\left(\frac{\Gamma(-\mathrm{i} \Lambda)}{\Gamma(1+\mathrm{i} \Lambda)}(-\mathrm{i} \Lambda)^{\mathrm{i} \sigma_{j}+B_{j} / 2}(\mathrm{i} \Lambda)^{\mathrm{i} \sigma_{j}-B_{j} / 2}\right)
$$

to the Coulomb branch expression for the enriched theory. The original partition function is thus a limit of the enriched partition function,

$$
Z(m, \widetilde{m}, z, \bar{z})=\lim _{\Lambda \rightarrow \infty}\left[\frac{1}{\gamma(-\mathrm{i} \Lambda)^{N\left(N_{f}-\widetilde{N}_{f}\right)}} Z_{\mathrm{enr}}\left(m,\{\widetilde{m}, \Lambda\}, z_{\text {bare }}, \bar{z}_{\text {bare }}\right)\right],
$$

where the factor $\gamma(-\mathrm{i} \Lambda)^{-N\left(N_{f}-\widetilde{N_{f}}\right)}$ has no physical effect, and the bare parameter $z_{\text {bare }}$ appearing in the enriched theory is related to the renormalized $z=z_{\text {ren }}$ (at the scale $\ell$ given by the equatorial radius of the squashed sphere) via

$$
z_{\text {bare }}=\frac{z_{\text {ren }}}{(-\mathrm{i} \Lambda)^{N_{f}-\widetilde{N}_{f}}} \quad \text { and } \quad \bar{z}_{\text {bare }}=\frac{\bar{z}_{\text {ren }}}{(\mathrm{i} \Lambda)^{N_{f}-\widetilde{N}_{f}}} .
$$

In particular, the FI parameter runs logarithmically, and the theta angle is shifted:

$$
\xi_{\text {ren }}=\xi_{\text {bare }}-\frac{1}{2 \pi}\left(N_{f}-\widetilde{N_{f}}\right) \ln \Lambda \quad \text { and } \quad \vartheta_{\text {ren }}=\vartheta_{\text {bare }}+\frac{\pi}{2}\left(N_{f}-\widetilde{N}_{f}\right) .
$$

Since the Coulomb branch representation involves an integral over arbitrarily large values of $\sigma \pm \mathrm{i} \frac{B}{2}$, our derivation of (2.76) above is not rigorous. However, one can split the integral into a region $\left|\sigma \pm \mathrm{i} \frac{B}{2}\right| \ll \Lambda$ and its complement, and check that the contribution from large $\sigma \pm \mathrm{i} \frac{B}{2}$ becomes negligible as $\Lambda \rightarrow \infty$. It is more convenient to perform such steps on the Higgs branch decomposition (2.58) of $Z_{\text {enr }}$ near 0 . Regardless of the value of $z$, the series expansions of vortex partition functions converges for $\Lambda$ large enough that $\left|z_{\text {bare }}\right|=|z| / \Lambda^{N_{f}-\widehat{N}_{f}}<1$. Then each term in the series for the enriched theory converges to the appropriate term for the $\widetilde{N_{f}}<N_{f}$ theory. Since the sum of terms with $\sum_{j=1}^{N} k_{j}>K$

\footnotetext{
${ }^{22}$ The dimensionful cutoff is $\Lambda / \ell$ in terms of the equatorial radius $\ell$ of the squashed two-sphere.
} 
decreases exponentially with $K$ in both series, $Z_{\mathrm{v}, \text { enr }}\left(z_{\text {bare }}\right) \rightarrow Z_{\mathrm{v}}(z)$. Other factors work out as for the Coulomb branch representation.

In the limit above, $N_{f}\left(N_{f}-\widetilde{N_{f}}\right)$ of the $N_{f}^{2}$ free hypermultiplets on $S_{b}^{4}$ become infinitely massive, and the corresponding factors must be removed from the enriched partition function to retain a finite result. The partition function of the surface operator with $\widetilde{N}_{f}<N_{f}$ in a theory of $N_{f} \widetilde{N_{f}}$ free hypermultiplets of masses (2.17) is thus the limit

$$
Z_{S^{2} \subset S_{b}^{4}}^{\mathrm{U}(N)}(z, \bar{z})=\lim _{\Lambda \rightarrow \infty}\left[\left(\frac{\prod_{s=1}^{N_{f}} \Upsilon\left(\frac{1}{b}\left(1+\mathrm{i} m_{s}+\mathrm{i} \Lambda\right)\right)}{\gamma(-\mathrm{i} \Lambda)^{N}}\right)^{N_{f}-\widetilde{N}_{f}} Z_{S^{2} \subset S_{b}^{4}, \text { enr }}^{\mathrm{U}(N)}\left(z_{\text {bare }}, \bar{z}_{\text {bare }}\right)\right]
$$

We now provide a Toda CFT interpretation of the limit for $N_{f}-\widetilde{N_{f}}=1$. For simplicity, label antifundamental multiplets of the enriched theory starting with the spectator multiplet, so that $\widetilde{m}_{1}=\Lambda \rightarrow \infty$. Replace the partition function of the enriched defect in (2.79) by its corresponding Toda CFT four-point function through the matching (2.51). After a conformal transformation which maps $(\infty, 1, x /(-\mathrm{i} \Lambda), 0)$ to $(0, x /(-\mathrm{i} \Lambda), 1, \infty)$,

$$
\begin{aligned}
Z_{S^{2} \subset S_{b}^{4}}^{\mathrm{U}(N)}(z, \bar{z})= & \lim _{\Lambda \rightarrow \infty}\left[A_{\mathrm{enr}}\left|\frac{x}{\Lambda}\right|^{2 \gamma_{0, \mathrm{enr}}-2 \Delta\left(\alpha_{0}\right)-2 \Delta\left(-b \omega_{N}\right)+2 \Delta\left(\alpha_{\infty}\right)+2 \Delta(\hat{m})}\left|1-\frac{x}{-\mathrm{i} \Lambda}\right|^{2 \gamma_{1, \mathrm{enr}}}\right. \\
& \left.\cdot \frac{\prod_{s=1}^{N_{f}} \Upsilon\left(\frac{1}{b}\left(1+\mathrm{i} m_{s}+\mathrm{i} \Lambda\right)\right)}{\gamma(-\mathrm{i} \Lambda)^{N}}\left\langle\widehat{V}_{\alpha_{0}}(\infty) \widehat{V}_{-b \omega_{N}}(1) \widehat{V}_{\hat{m}}\left(\frac{x}{-\mathrm{i} \Lambda}, \frac{\bar{x}}{\mathrm{i} \Lambda}\right) \widehat{V}_{\alpha_{\infty}}(0)\right\rangle\right]
\end{aligned}
$$

with $x=(-1)^{N_{f}+N-1} z$, and parameters $\alpha_{0}, \hat{m}=(\varkappa+N b) h_{1}, \alpha_{\infty}, A_{\mathrm{enr}}, \gamma_{0, \mathrm{enr}}$ and $\gamma_{1, \mathrm{enr}}$ given below (2.51). As $\Lambda \rightarrow \infty, \gamma_{1, \text { enr }} \sim \frac{N}{N_{f}} \mathrm{i} \Lambda$, thus $|1-x /(-\mathrm{i} \Lambda)|^{2 \gamma_{1, \mathrm{enr}}} \rightarrow e^{\left(N / N_{f}\right)(x+\bar{x})}$.

In the same limit, the punctures $\widehat{V}_{\hat{m}}$ and $\widehat{V}_{\alpha_{\infty}}$ collide, with momenta growing as the inverse of the distance, keeping a constant sum $c_{0}+N b h_{1}=(\varkappa+N b) h_{1}+\alpha_{\infty}$ given in (2.84). We study such collision limits in appendix A.6 and define (A.113)

$$
\begin{aligned}
& \widehat{\mathbb{V}}_{c_{0}+N b h_{1} ;-(x / b) h_{1},(\bar{x} / b) h_{1}}(0)=\lim _{\Lambda \rightarrow \infty}\left[\Upsilon\left(\varkappa+N b+\left\langle Q-c_{0}-N b h_{1}, h_{1}\right\rangle\right)^{N_{f}}\right. \\
& \left.\cdot\left[\frac{\Lambda}{b}\right]^{\langle Q, Q\rangle-2 \Delta\left(c_{0}+N b h_{1}\right)}\left|\frac{x}{\Lambda}\right|^{2\left\langle(\varkappa+N b) h_{1}, c_{0}-\varkappa h_{1}\right\rangle} \widehat{V}_{(\varkappa+N b) h_{1}}\left(\frac{x}{-\mathrm{i} \Lambda}, \frac{\bar{x}}{\mathrm{i} \Lambda}\right) \widehat{V}_{c_{0}-\varkappa h_{1}}(0)\right]_{\varkappa \sim \mathrm{i} \Lambda / b} .
\end{aligned}
$$

The Upsilon functions and gamma functions in (2.80) and (2.81) can be recast in the same form through the asymptotics (A.11), (A.10), and $\gamma(1+\mathrm{i} \Lambda+a) \sim \gamma(1+\mathrm{i} \Lambda) \Lambda^{2 a}$. Let $\mathrm{i} \underline{m}=\frac{1}{N_{f}} \sum_{s=1}^{N_{f}} \mathrm{i} m_{s}$. Then,

$$
\begin{gathered}
\gamma(1+\mathrm{i} \Lambda)^{N} \prod_{s=1}^{N_{f}} \Upsilon\left(\frac{1}{b}\left(1+\mathrm{i} \Lambda+\mathrm{i} m_{s}\right)\right)=\gamma(1+\mathrm{i} \Lambda)^{N} \prod_{s=1}^{N_{f}} \Upsilon\left(\frac{1}{b}(1+\mathrm{i} \Lambda+i \underline{m})+\left\langle Q-\alpha_{0}, h_{s}\right\rangle\right) \\
\sim \gamma(1+\mathrm{i} \Lambda)^{N} \Upsilon\left(\frac{1}{b}(1+\mathrm{i} \Lambda+\mathrm{i} \underline{m})\right)^{N_{f}}[\Lambda / b]^{\langle Q, Q\rangle-2 \Delta\left(\alpha_{0}\right)} \\
\sim \Upsilon\left(\frac{1}{b}(1+\mathrm{i} \Lambda+\mathrm{i} \underline{m})+b N / N_{f}\right)^{N_{f}} b^{N+2 N \mathrm{i} \Lambda}[\Lambda / b]^{\langle Q, Q\rangle-2 \Delta\left(\alpha_{0}\right)-2 N \underline{\mathrm{i}} \underline{m}+N\left(N_{f}-N\right) b^{2} / N_{f}} .
\end{gathered}
$$


The last Upsilon functions are precisely those appearing in (2.81). Plugging back into (2.80), all powers of $\Lambda$ and $b^{\Lambda}$ cancel, and we can drop the limit.

All in all, the partition function of a surface operator describing a $\mathrm{U}(N)$ vector multiplet with $N_{f}$ fundamental and $\widetilde{N}_{f}=N_{f}-1$ antifundamental chiral multiplets, coupled to $N_{f}\left(N_{f}-1\right)$ hypermultiplets on $S_{b}^{4}$ is equal to a Toda CFT correlator with an antisymmetric degenerate insertion and a rank 1 irregular puncture: ${ }^{23}$

$$
Z_{S^{2} \subset S_{b}^{4}}^{\mathrm{U}(N), N_{f}, N_{f}-1}(z, \bar{z})=A|x|^{2 \gamma_{0}} e^{\frac{N}{N_{f}}(x+\bar{x})}\left\langle\widehat{V}_{\alpha_{0}}(\infty) \widehat{V}_{-b \omega_{N}}(1) \widehat{\mathbb{V}}_{c_{0}+N b h_{1} ; c_{1}, \bar{c}_{1}}(0)\right\rangle .
$$

As before, $x=(-1)^{N_{f}+N-1} z$. The irregular puncture $\widehat{\mathbb{V}}$ is defined above and studied in appendix A.6. The momenta $c_{0}, c_{1}, \bar{c}_{1}$, and $\alpha_{0}$ are

$$
\begin{aligned}
& c_{0}=Q+\frac{1}{b} \sum_{s=1}^{N_{f}}\left(1+\mathrm{i} m_{s}\right) h_{1}+\frac{1}{b} \sum_{s=2}^{N_{f}} \mathrm{i} \widetilde{m}_{s}\left(h_{1}-h_{s}\right), \\
& c_{1}=-\frac{x}{b} h_{1}, \quad \bar{c}_{1}=\frac{\bar{x}}{b} h_{1}, \quad \alpha_{0}=Q-\frac{1}{b} \sum_{s=1}^{N_{f}} \mathrm{i} m_{s} h_{s},
\end{aligned}
$$

and the constant $A$ and exponent $\gamma_{0}$ are ${ }^{24}$

$$
\begin{aligned}
& A=b^{N\left(N_{f}-1\right)\left(b^{2}+1\right)+2 \Delta\left(\alpha_{0}\right)-2 \Delta\left(c_{0}\right)}, \\
& \gamma_{0}=\Delta\left(c_{0}\right)-\Delta\left(\alpha_{0}\right)-N \sum_{s=1}^{N_{f}} \mathrm{i} m_{s}-N \sum_{s=2}^{N_{f}}\left(1+\mathrm{i} \widetilde{m}_{s}\right)-\frac{N(N-1)}{2} b^{2} .
\end{aligned}
$$

As we have seen, it is natural from the gauge theory point of view to decouple further antifundamental chiral multiplets by making them massive. Specifically, from (2.79) we know that the partition function of a surface operator described by a $\mathrm{U}(N)$ vector multiplet coupled to $N_{f}$ fundamental and $\widetilde{N_{f}}=N_{f}-k \leq N_{f}-2$ antifundamental chiral multiplets is a limit of $Z_{S^{2} \subset S_{b}^{4}}^{\mathrm{U}(N), N_{f}, N_{f}-1}\left(z /(-\mathrm{i} \Lambda)^{k-1}, \bar{z} /(\mathrm{i} \Lambda)^{k-1}\right)$ with twisted masses $\widetilde{m}_{2}=\cdots=\widetilde{m}_{k}=\Lambda$, multiplied by some factor. On the Toda CFT side of the matching (2.83), the limit amounts to taking $\left\langle c_{0}, h_{s}\right\rangle \sim \mathrm{i} \Lambda / b$ for $2 \leq s \leq k$ and letting $c_{1}$ and $\bar{c}_{1}$ decrease as $\Lambda^{-(k-1)}$. Such a limit does not fit in the framework described in appendix A.6, since the parameter $c_{0}$ blows up. However, translating the gauge theory factors to the Toda CFT and setting $N=0$ for simplicity, we find that the two-point function of a generic vertex operator $\widehat{V}_{\alpha_{0}}$ with

$$
\left[|\nu|^{2 \Delta\left(c_{0}\right)-\langle Q, Q\rangle} \prod_{t=2}^{k} \Upsilon\left(\left\langle Q-c_{0}, h_{t}\right\rangle\right)^{N_{f}} \widehat{\mathbb{V}}_{c_{0} ;-\nu h_{1}, \bar{\nu} h_{1}}\right]_{\substack{\nu=x /\left[b(-\mathrm{i} \Lambda)^{k-1}\right] \\ c_{0} \sim \mathrm{i} \Lambda\left(k h_{1}-\omega_{k}\right)}}
$$

\footnotetext{
${ }^{23}$ Following the argument below (2.10), the factor $A|x|^{2 \gamma_{0}} e^{\left(N / N_{f}\right)(x+\bar{x})}$ can be absorbed in the $S^{2}$ partition function. We keep the factor explicitly to compare gauge theory and Toda CFT results.

${ }^{24}$ Mapping $\{0,1, \infty\}$ to $\{\infty, x, 0\}$ gives a closer analogue of the $\widetilde{N_{f}}=N_{f}$ matching. This replaces $\gamma_{0}$ by the simpler $\gamma_{0}-\Delta\left(c_{0}+N b h_{1}\right)+\Delta\left(\alpha_{0}\right)+\Delta\left(-b \omega_{N}\right)=-\frac{N}{N_{f}} \sum_{s=1}^{N_{f}} \mathrm{i} m_{s}-\frac{N\left(N_{f}-N\right)}{2}\left(b^{2}+1\right)$. However, the transformation properties (A.98) of rank 1 irregular punctures would make the parameters $c_{1}$ and $\bar{c}_{1}$ infinite. The best convention to cancel this infinity is not clear.
} 
remains finite as $\Lambda \rightarrow \infty$. This suggests that the operator (2.87) itself has a limit. Additionally, the OPE (A.93) of the stress-energy tensor with a rank 1 puncture includes a term $\Delta\left(c_{0}\right)+\left\langle c_{1}, \partial_{c_{1}}\right\rangle$, and the normalization factor $|\nu|^{2 \Delta\left(c_{0}\right)}$ ensures that the singular term $\Delta\left(c_{0}\right)$ is absorbed in $\left\langle c_{1}, \partial_{c_{1}}\right\rangle$. Unfortunately, it is difficult to go further, as the OPE with higher currents of the $W_{N_{f}}$ algebra contain many singular terms, and all must be carefully canceled by the choice of normalization before taking the limit.

Having dissected the partition function of theories with fundamental and antifundamental matter, we consider next theories with an adjoint chiral multiplet.

\subsection{SQCDA and Toda symmetric degenerate}

We focus in this section on $\mathcal{N}=(2,2)$ SQCDA: a $\mathrm{U}(N)$ vector multiplet coupled to an adjoint chiral multiplet $X$ and $N_{f}$ fundamental and $N_{f}$ antifundamental chiral multiplets. Twisted masses (plus $R$-charges) are $m_{X}, m_{s}$, and $\widetilde{m}_{s}$. This theory, coupled to $N_{f}^{2}$ hypermultiplets with masses given by (2.17), defines a surface operator. We equate the $S^{2} \subset S_{b}^{4}$ partition function of the $4 \mathrm{~d} / 2 \mathrm{~d}$ system to a Toda CFT correlator with a degenerate field $\widehat{V}_{-N b h_{1}}$ labeled by the $N$-th symmetric representation of $A_{N_{f}-1}$. Namely, we check that ${ }^{25}$

$$
Z_{S^{2} \subset S_{b}^{4}}^{\mathrm{U}(N) \operatorname{SQCDA}}\left(m, \widetilde{m}, m_{X}, z, \bar{z}\right)=A|y|^{2 \gamma_{0}}|1-y|^{2 \gamma_{1}}\left\langle\widehat{V}_{\alpha_{\infty}}(\infty) \widehat{V}_{\hat{m}}(1) \widehat{V}_{-N b h_{1}}(y, \bar{y}) \widehat{V}_{\alpha_{0}}(0)\right\rangle
$$

with $y=(-1)^{N_{f}} z \operatorname{and}^{26} b^{2}=\mathrm{i} m_{X}$, momenta

$$
\begin{aligned}
\alpha_{0}=Q-\frac{1}{b} \sum_{s=1}^{N_{f}} \mathrm{i} m_{s} h_{s}, & \hat{m}=(\varkappa+N b) h_{1}, \\
\alpha_{\infty}=Q-\frac{1}{b} \sum_{s=1}^{N_{f}} \mathrm{i} \widetilde{m}_{s} h_{s}, & \varkappa=\frac{1}{b} \sum_{s=1}^{N_{f}}\left(1+\mathrm{i} m_{s}+\mathrm{i} \widetilde{m}_{s}\right),
\end{aligned}
$$

and coefficients

$$
\begin{aligned}
\gamma_{0} & =-\frac{N}{N_{f}} \sum_{s=1}^{N_{f}} \mathrm{i} m_{s}-\frac{N\left(N_{f}-1\right)}{2}\left(b^{2}+1\right)-\frac{N(N-1)}{2} b^{2} \\
\gamma_{1} & =-\frac{N\left(N_{f}-N\right)}{N_{f}} b^{2}+\frac{N}{N_{f}} \sum_{s=1}^{N_{f}}\left(\mathrm{i} m_{s}+\mathrm{i} \widetilde{m}_{s}\right), \\
A & =b^{N N_{f}\left(1+b^{2}\right)-N^{2} b^{2}-2 N b \varkappa} \prod_{\nu=1}^{N} \gamma\left(-\nu b^{2}\right) .
\end{aligned}
$$

We recognize the same symmetries as for SQED and SQCD, under permutations of the $m_{s}$ or the $\widetilde{m}_{s}$, and under $z \leftrightarrow \frac{1}{z}$ and exchanging those two sets of masses. Setting $N=1$

\footnotetext{
${ }^{25}$ As explained below (2.10), the factor $A|x|^{2 \gamma_{0}}|1-x|^{2 \gamma_{1}}$ can be absorbed into the partition function. To compare gauge theory and Toda CFT results it is best to keep the factor explicitly.

${ }^{26}$ The full flavour group of SQCDA is $\mathrm{U}(1) \times S\left[\mathrm{U}\left(N_{f}\right) \times \mathrm{U}\left(N_{f}\right)\right]$, where the factors act on the adjoint, fundamental, and antifundamental chiral multiplets. The relation $b^{2}=\mathrm{i} m_{X}$ identifies the first $\mathrm{U}(1)$ flavour symmetry with rotations transverse to the surface operator.
} 
reproduces the matching (2.19) of SQED, but $A$ has an additional factor of $\gamma\left(-b^{2}\right)=$ $\gamma\left(-\mathrm{i} m_{X}\right)$ : this is the one-loop determinant of the adjoint chiral multiplet, which decouples in an abelian theory.

Given the geometrical origin of the deformation parameter, one has $b^{2}>0$. On the other hand, the $S^{2}$ partition function is defined with positive $R$-charges $\operatorname{Re}(-2 \mathrm{i} m)$. The two requirements are incompatible with $b^{2}=\mathrm{i} m_{X}$, hence one of those two parameters must be continued beyond its usual range. For now, we analytically continue the $R$-charge: it is easier because the partition function depends holomorphically on $\mathrm{i} m_{X}$, as deduced from explicit expressions. However, we will encounter in section 2.3.3 a setting where $b^{2}=\mathrm{i} m_{X}$ is fixed to a real negative value. Given that the Upsilon function which appears in $Z_{S_{b}^{4}}^{\text {free }}$ and in Toda correlators cannot be continued to negative $b^{2}$, we will have to first recast the relation (2.88) in the form $Z_{S^{2}}=\langle\cdots\rangle / Z_{S_{b}^{4}}^{\text {free }}$ for the analytic continuation in $b$ to make sense.

Once more, we fix the dictionary and demonstrate the equality by comparing exponents in the s-, $\mathrm{t}$ - and $\mathrm{u}$-channels. The equality of Toda CFT three-point functions and gauge theory one-loop determinants (for the s- and u-channels) is checked in appendix A.2, and the expression of $A$ is found there.

The Coulomb branch representation reads

$$
\begin{aligned}
& Z^{\mathrm{SQCDA}}=\frac{1}{N !} \sum_{B \in \mathbb{Z}^{N}} \int_{\mathbb{R}^{N}} \frac{\mathrm{d}^{N} \sigma}{(2 \pi)^{N}}\left\{\left[(-1)^{N-1} z\right]^{\operatorname{Tr}\left(\mathrm{i} \sigma+\frac{B}{2}\right)}\left[(-1)^{N-1} \bar{z}\right]^{\operatorname{Tr}\left(\mathrm{i} \sigma-\frac{B}{2}\right)}\right. \\
& \cdot \prod_{i<j}\left[\left(\sigma_{i}-\sigma_{j}\right)^{2}+\frac{\left(B_{i}-B_{j}\right)^{2}}{4}\right] \prod_{j=1}^{N} \prod_{s=1}^{N_{f}}\left[\frac{\Gamma\left(-\mathrm{i} m_{s}-\mathrm{i} \sigma_{j}-\frac{B_{j}}{2}\right)}{\Gamma\left(1+\mathrm{i} m_{s}+\mathrm{i} \sigma_{j}-\frac{B_{j}}{2}\right)} \frac{\Gamma\left(-\mathrm{i} \widetilde{m}_{s}+\mathrm{i} \sigma_{j}+\frac{B_{j}}{2}\right)}{\Gamma\left(1+\mathrm{i} \widetilde{m}_{s}-\mathrm{i} \sigma_{j}+\frac{B_{j}}{2}\right)}\right] \\
& \left.\cdot \prod_{i=1}^{N} \prod_{j=1}^{N}\left[\frac{\Gamma\left(-\mathrm{i} m_{X}-\mathrm{i} \sigma_{i}+\mathrm{i} \sigma_{j}-\frac{B_{i}-B_{j}}{2}\right)}{\Gamma\left(1+\mathrm{i} m_{X}+\mathrm{i} \sigma_{i}-\mathrm{i} \sigma_{j}-\frac{B_{i}-B_{j}}{2}\right)}\right]\right\} .
\end{aligned}
$$

We will expand this partition function around the points $0,(-1)^{N_{f}}$ and $\infty$, where, as a function of $z$, it has branch points. This follows the path we traced for SQED: the behaviors near $z=0$ and $\infty$ are probed by closing integration contours towards $\pm \mathrm{i} \infty$. The partition function is then expressed as a sum over poles of the integrand, which are characterized up to integers by the set of Gamma functions which are singular for those values of $\mathrm{i} \sigma$. The behavior near $(-1)^{N_{f}}$ is found by splitting the Coulomb branch integral depending on whether each $\left|\sigma_{j} \pm \frac{\mathrm{i} B_{j}}{2}\right| \lessgtr \ln |z|$.

\subsubsection{Expanding the SQCDA partition function}

We start with the s-channel expansion for $|z|<1$. Ignoring for a moment the magnetic flux $B$, and integer shifts due to the infinite set of poles of the Gamma function, we find that poles enclosed by the contour must be such that each $\mathrm{i} \sigma_{j}$ is either $-\mathrm{i} m_{s}$ for some flavour $s$, or $\mathrm{i} \sigma_{i}-\mathrm{i} m_{X}$ for some other color $i$. As in the case of SQCD, the vector multiplet one-loop determinant enforces $\mathrm{i} \sigma_{i} \neq \mathrm{i} \sigma_{j}$ for any two distinct colors, hence $\left\{\mathrm{i} \sigma_{j}\right\}$ is $\left\{-\mathrm{i} m_{s}-\mu \mathrm{i} m_{X} \mid\right.$ $\left.1 \leq s \leq N_{f}, 0 \leq \mu<n_{s}\right\}$ for some choice of integers $n_{s}$ with $n_{1}+\cdots+n_{N_{f}}=N$. It is convenient to label colors with indices $(s, \mu)$ instead of $j \in \llbracket 1, N \rrbracket$, and denote $I=\{(s, \mu)\}$. 
The sums over $B$ and over poles of Gamma functions introduce shifts, in the form of sums over $2 N$ integers $k_{s \mu}^{ \pm} \geq 0$, and poles are

$$
\mathrm{i} \sigma_{s \mu} \pm \frac{B_{s \mu}}{2}=-\mathrm{i} m_{s}-\mu \mathrm{i} m_{X}+k_{s \mu}^{ \pm}
$$

for $(s, \mu) \in I$. The partition function can then be recast as a sum over residues at those values of $\mathrm{i} \sigma \pm \frac{B}{2}$. It turns out that the residues vanish unless $k_{s \mu}^{ \pm} \leq k_{s(\mu+1)}^{ \pm}$for every $(s, \mu) \in I$ and sign \pm : this indicates that (2.94) also labels some points which are not poles; thankfully, the residue formula is robust against such overcounting.

Since every factor in the Coulomb branch formula depends only on $\mathrm{i} \sigma+\frac{B}{2}$ hence on $k^{+}$, or on $\mathrm{i} \sigma-\frac{B}{2}$ hence on $k^{-}$, the series over $k^{+}$and over $k^{-}$decouple, and $Z^{\mathrm{SQCDA}}$ splits into a sum of factorized terms labeled by the choice of $\left\{n_{s}\right\}$,

$$
\begin{aligned}
Z_{S^{2}}^{\mathrm{SQCDA}}= & \sum_{n_{1}+\cdots+n_{N_{f}}=N}(z \bar{z})^{\sum_{(s, \mu) \in I}\left(-\mathrm{i} m_{s}-\mu \mathrm{i} m_{X}\right)} Z_{11,\{n\}} Z_{\mathrm{v},\{n\}}\left[(-1)^{N_{f}} z\right] Z_{\mathrm{v},\{n\}}\left[(-1)^{N_{f}} \bar{z}\right] \\
Z_{11,\{n\}}= & \prod_{(s, \mu) \in I} \prod_{t=1}^{N_{f}} \frac{\gamma\left(-\mathrm{i} m_{t}-n_{t} \mathrm{i} m_{X}+\mathrm{i} m_{s}+\mu \mathrm{i} m_{X}\right)}{\gamma\left(1+\mathrm{i} \widetilde{m}_{t}+\mathrm{i} m_{s}+\mu \mathrm{i} m_{X}\right)} \\
Z_{\mathrm{v},\{n\}}(y)= & \sum_{k: I \rightarrow \mathbb{Z}_{\geq 0}} \prod_{(s, \mu) \in I}\left[y^{k_{s \mu}} \prod_{t=1}^{N_{f}} \frac{\left(-\mathrm{i} \widetilde{m}_{t}-\mathrm{i} m_{s}-\mu \mathrm{i} m_{X}\right)_{k_{s \mu}}}{\left(1+\mathrm{i} m_{t}-\mathrm{i} m_{s}+\left(n_{t}-\mu\right) \mathrm{i} m_{X}\right)_{k_{s \mu}}}\right. \\
& \left.\cdot \frac{\prod_{t=1}^{N_{f}}\left(1+\mathrm{i} m_{t}-\mathrm{i} m_{s}+\left(n_{t}-\mu\right) \mathrm{i} m_{X}+k_{s \mu}-k_{t\left(n_{t}-1\right)}\right)_{\left.k_{t(n t}-1\right)}}{\prod_{(t, \nu) \in I}\left(1+\mathrm{i} m_{t}-\mathrm{i} m_{s}+(\nu-\mu) \mathrm{i} m_{X}+k_{s \mu}-k_{t \nu}\right)_{k_{t \nu}-k_{t(\nu-1)}}}\right]
\end{aligned}
$$

where we define $k_{t,-1}=0$ for convenience. Carrying through the same procedure for $|z|>1$ yields a u-channel decomposition similar to the s-channel decomposition (2.95), with $m_{s} \leftrightarrow \widetilde{m}_{s}, z \rightarrow z^{-1}$ and $\bar{z} \rightarrow \bar{z}^{-1}$.

Having found powers of $|z|$ in the s-channel and u-channel decompositions of $Z^{\mathrm{SQCDA}}$, we now expand the Coulomb branch integral in the t-channel. The first step is to use the identity $\frac{\Gamma(-\mathrm{i} a-B / 2)}{\Gamma(1+\mathrm{i} a-B / 2)}=(-1)^{B} \frac{\Gamma(-\mathrm{i} a+B / 2)}{\Gamma(1+\mathrm{i} a+B / 2)}$ on the one-loop determinants of fundamental chiral multiplets, and on half of the Gamma functions stemming from the adjoint chiral multiplet, and absorb the resulting signs into

$$
y=(-1)^{N_{f}} z \quad \text { and } \quad \bar{y}=(-1)^{N_{f}} \bar{z} .
$$

The integrand resulting from this operation can be recast as

$$
\begin{gathered}
y^{\operatorname{Tr}\left(\mathrm{i} \sigma+\frac{B}{2}\right)} \bar{y}^{\operatorname{Tr}\left(\mathrm{i} \sigma-\frac{B}{2}\right)} \prod_{j=1}^{N} \prod_{s=1}^{N_{f}}\left[\frac{\Gamma\left(-\mathrm{i} m_{s}-\mathrm{i} \sigma_{j}+\frac{B_{j}}{2}\right)}{\Gamma\left(1+\mathrm{i} \widetilde{m}_{s}-\mathrm{i} \sigma_{j}+\frac{B_{j}}{2}\right)} \frac{\Gamma\left(-\mathrm{i} \widetilde{m}_{s}+\mathrm{i} \sigma_{j}+\frac{B_{j}}{2}\right)}{\Gamma\left(1+\mathrm{i} m_{s}+\mathrm{i} \sigma_{j}+\frac{B_{j}}{2}\right)}\right] \\
\cdot \gamma\left(-\mathrm{i} m_{X}\right)^{N} \prod_{ \pm} \prod_{i<j}^{N}\left[\frac{\left( \pm\left(\mathrm{i} \sigma_{i}-\mathrm{i} \sigma_{j}\right)+\frac{B_{i}-B_{j}}{2}\right) \Gamma\left(-\mathrm{i} m_{X} \pm\left(\mathrm{i} \sigma_{i}-\mathrm{i} \sigma_{j}\right)+\frac{B_{i}-B_{j}}{2}\right)}{\Gamma\left(1+\mathrm{i} m_{X} \pm\left(\mathrm{i} \sigma_{i}-\mathrm{i} \sigma_{j}\right)+\frac{B_{i}-B_{j}}{2}\right)}\right]
\end{gathered}
$$

by writing the vector multiplet one-loop determinant as a product of $\pm\left(\mathrm{i} \sigma_{i}-\mathrm{i} \sigma_{j}\right)+\frac{B_{i}-B_{j}}{2}$. We now split the sums and integrals in the same way as for SQED on page 21, one 
pair $\left(\sigma_{j}, B_{j}\right)$ at a time. For $\left|\mathrm{i} \sigma_{j}+\frac{B_{j}}{2}\right|<|\ln y|^{-1}$, we expand the classical contribution $y^{\mathrm{i} \sigma_{j}+\frac{B_{j}}{2}} \bar{y}^{\mathrm{i} \sigma_{j}-\frac{B_{j}}{2}}$ as a series in $\ln y$ and $\ln \bar{y}$; the integral and sum only contributes a constant factor. For $\left|\mathrm{i} \sigma_{j}+\frac{B_{j}}{2}\right|>|\ln y|^{-1}$, the sum over $B_{j}$ is well approximated by an integral, and we expand the Gamma functions which involve this particular combination as a power of $\left|\mathrm{i} \sigma_{j}+\frac{B_{j}}{2}\right|$ times a power series in $\left(\mathrm{i} \sigma_{j} \pm \frac{B_{j}}{2}\right)^{-1}$. Rescaling $\mathrm{i} \sigma_{j}+\frac{B_{j}}{2}$ by $\ln y$ makes the classical contribution independent of $y$, and extracting a power of $|\ln y|$ leaves a series in $\ln y$ and $\ln \bar{y}$ as the sole dependence in $y$. After performing this procedure for all pairs $\left(\sigma_{j}, B_{j}\right)$, we obtain $2^{N}$ contributions, labeled by the set $K \subseteq\{1, \ldots, N\}$ of colors $j$ such that $\left|\mathrm{i} \sigma_{j}+\frac{B_{j}}{2}\right|>|\ln y|^{-1}$ is large. The contribution for a given set $K$ behaves as

$$
Z_{K} \sim|1-y|^{-2 k+2 k \sum_{s=1}^{N_{f}}\left(1+\mathrm{i} m_{s}+\mathrm{i} \widetilde{m}_{s}\right)+2 k[2 N-k-1] \mathrm{i} m_{X}},
$$

multiplied by a constant and by a series in powers of $1-y$ and $1-\bar{y}$, where $k=\# K$ is the number of elements in $K$ and we used $(\ln y)^{\alpha}=(1-y)^{\alpha} \cdot($ series). There are $N+1$ distinct exponents, corresponding to values $k \in \llbracket 0, N \rrbracket$. This approach does not seem amenable to finding multiplicities attached to each power of $1-y$, hence we will not be able to probe that aspect of the correspondence.

\subsubsection{Matching parameters for SQCDA}

We are now ready to match the gauge theory data to Toda CFT data. The s- and u-channel decompositions of $Z^{\mathrm{SQCDA}}$ have

$$
\left(\begin{array}{c}
N_{f}+N-1 \\
N
\end{array}\right)=\operatorname{dim}\left(\mathcal{R}\left(N h_{1}\right)\right)
$$

terms, which is the dimension of the $N$-th symmetric representation $\mathcal{R}\left(N h_{1}\right)$ of $A_{N_{f}-1}$, with highest weight $N h_{1}$. Thus, in analogy with SQCD, we expect $Z^{\mathrm{SQCDA}}$ to match a Toda four-point correlation function involving the degenerate operator $\widehat{V}_{-N b h_{1}}$. The fusion rule then allows shifts of generic momenta by $-b h=-b \sum_{s=1}^{N_{f}} n_{s} h_{s}$ for a choice of integers $n_{1}+\cdots+n_{N_{f}}=N$. We thus wish to match the s-channel exponents

$$
\Delta\left(\alpha_{0}-b h\right)-\Delta\left(\alpha_{0}\right)-\Delta\left(-N b h_{1}\right)+\gamma_{0}=-\sum_{s=1}^{N_{f}}\left[n_{s} \mathrm{i} m_{s}+\frac{n_{s}\left(n_{s}-1\right)}{2} \mathrm{i} m_{X}\right] .
$$

This equality holds if $\mathrm{i} m_{X}=b^{2}$, and $\alpha_{0}$ and $\gamma_{0}$ are as given in (2.89) and (2.90). The u-channel powers are similar,

$$
\Delta\left(\alpha_{\infty}\right)-\Delta\left(\alpha_{\infty}-b h\right)-\Delta\left(-N b h_{1}\right)+\gamma_{0}+\gamma_{1}=\sum_{s=1}^{N_{f}}\left[n_{s} \mathrm{i} \tilde{m}_{s}+\frac{n_{s}\left(n_{s}-1\right)}{2} \mathrm{i} m_{X}\right]
$$

and the equality holds for values of $\alpha_{\infty}$ and $\gamma_{1}$ in (2.89) and (2.91).

We find in appendix A.4 that the fusion of $(\varkappa+N b) h_{1}$ with $-N b h_{1}$ allows the t-channel internal momenta $(\varkappa+n b) h_{1}-n b h_{2}$ for $0 \leq n \leq N$. This fusion rule (A.64) provides the 
powers of $1-y$ for the t-channel of the Toda correlator, and power matching then requires

$$
\begin{aligned}
\Delta\left((\varkappa+n b) h_{1}-n b h_{2}\right)- & \Delta\left((\varkappa+N b) h_{1}\right)-\Delta\left(-N b h_{1}\right)+\gamma_{1} \\
& =k\left[\sum_{s=1}^{N_{f}}\left(\mathrm{i} m_{s}+\mathrm{i} \widetilde{m}_{s}\right)+\left(N_{f}-1\right)+(2 N-k-1) \mathrm{i} m_{X}\right] .
\end{aligned}
$$

The exponents are equal if $n=N-k$, and $\varkappa$ is as given in (2.89).

Finally, as checked in appendix A.2, the Toda CFT three-point functions which appear in the s-channel decomposition of the correlator reproduce the corresponding one-loop determinants in (2.95), provided $A$ is as given in (2.92). For any given $N$, the techniques of appendix A.3 can yield the Toda CFT braiding matrix of $\widehat{V}_{-N b h_{1}}$ with $\widehat{V}_{\hat{m}}$. However, we did not find a closed form of those matrices or their gauge theory analogues to provide a proof of the matching (2.88).

\subsubsection{Adding a superpotential to SQCDA}

We now discuss the effect of adding to SQCDA a superpotential term of the form $W=\sum_{t=1}^{N_{f}} \widetilde{q}_{t} X^{l_{t}} q_{t}$ or $W=\operatorname{Tr} X^{l+1}$, where $q_{t}, \widetilde{q}_{t}$, and $X$ denote the fundamental, antifundamental, and adjoint chiral multiplets, and $l_{t}$ and $l$ are non-negative integers.

The deformation term which localizes to the Higgs branch of the theory with no superpotential can still be used in the presence of a superpotential, and it yields the same decomposition into vortex and anti-vortex partition functions. Hence, the only effect of the superpotential on the partition function is to constrain the (complexified) twisted masses of chiral multiplets. On the other hand, the superpotential term is in fact $\mathcal{Q}$-exact for the choice of localization supercharge $\mathcal{Q}$, thus one can include it into the deformation term. This lifts some vacua of the deformation term through F-term constraints, thus removes some terms from the sum over Higgs branch vacua. The two deformation terms must yield equal results for the partition function. Therefore, the terms forbidden by F-term constraints must vanish in the larger sum: they must have zero one-loop determinant. As a result, we can either solve D-term and F-term equations to find vacua of the enhanced deformation term, or remove vacua of the original deformation term whose one-loop determinant vanishes when imposing the superpotential constraint on $R$-charges.

First, we focus on a generalization of the superpotential $\widetilde{q} X q$ of $\mathcal{N}=(2,2)^{*} \mathrm{SQCD},{ }^{27}$

$$
W=\sum_{t=1}^{N_{f}} \widetilde{q}_{t} X^{l_{t}} q_{t},
$$

where $l_{t} \geq 0$ is an integer for each flavour $1 \leq t \leq N_{f}$. We let $L=\sum_{t=1}^{N_{f}} l_{t}$. The superpotential must have a total $R$-charge of 2 and a vanishing twisted mass, hence i $\widetilde{m}_{t}+$ $l_{t} \mathrm{i} m_{X}+\mathrm{i} m_{t}=-1$ for each $1 \leq t \leq N_{f}$. The one-loop determinant (2.96) then contains a vanishing factor $1 / \gamma\left(1+\mathrm{i} \widetilde{m}_{t}+\mathrm{i} m_{t}+l_{t} \mathrm{i} m_{X}\right)=0$ whenever any $n_{t}>l_{t}$, thus those terms do not contribute to the partition function. An equivalent point of view is that the corresponding Higgs branch vacua have $X^{n_{t}-1} q_{t} \neq 0$ and are forbidden by the F-term

\footnotetext{
${ }^{27} \mathcal{N}=(2,2)^{*}$ SQCD is the mass deformation of $\mathcal{N}=(4,4)$ SQCD.
} 
equation $X^{l_{t}} q_{t}=0$. Terms in the Higgs branch representation of the partition function are thus labeled by integers $0 \leq n_{t} \leq l_{t}$ with $\sum_{t=1}^{N_{f}} n_{t}=N$. Note that $n_{t} \leq l_{t}$ implies $N \leq L$, analogous to the condition $N \leq N_{f}$ for SQCD.

The constraint on (complexified) twisted masses translates to a constraint on the momenta of operators appearing in the corresponding Toda CFT correlator. The semidegenerate operator becomes degenerate:

$$
\hat{m}=\left[\frac{1}{b} \sum_{t=1}^{N_{f}}\left(1+\mathrm{i} m_{t}+\mathrm{i} \widetilde{m}_{t}\right)+N b\right] h_{1}=-(L-N) b h_{1},
$$

where we used $\mathrm{i} m_{X}=b^{2}$. Thus, the outgoing momentum $2 Q-\alpha_{\infty}$ must take the form $\alpha_{0}-b h-b h^{\prime}$, where $h=\sum_{t} n_{t} h_{t}$ is a weight of $\mathcal{R}\left(N h_{1}\right)$ and $h^{\prime}=\sum_{t} n_{t}^{\prime} h_{t}$ is a weight of $\mathcal{R}\left((L-N) h_{1}\right)$. The superpotential ensures that this is the case:

$$
2 Q-\alpha_{\infty}=Q+\frac{1}{b} \sum_{t=1}^{N_{f}} \mathrm{i} \widetilde{m}_{t} h_{t}=Q-\frac{1}{b} \sum_{t=1}^{N_{f}}\left(\mathrm{i} m_{t}+l_{t} b^{2}+1\right) h_{t}=\alpha_{0}-b \sum_{t=1}^{N_{f}} l_{t} h_{t} .
$$

The conformal block decomposition contains one term for each way of splitting $\sum_{t} l_{t} h_{t}$ into a sum $h+h^{\prime}$ of weights of $\mathcal{R}\left(N h_{1}\right)$ and $\mathcal{R}\left((L-N) h_{1}\right)$, that is, each set of integers $0 \leq n_{t} \leq l_{t}$ with $\sum_{t} n_{t}=N$. In section 3.2.1, we note that the vertex operators $\widehat{V}_{-(L-N) b h_{1}}$ and $\widehat{V}_{-N b h_{1}}$ have the same form with $N \leftrightarrow L-N$, and deduce a duality between theories with gauge groups $\mathrm{U}(N)$ and $\mathrm{U}(L-N)$. This duality reduces when all $l_{t}=1$ to an $\mathcal{N}=(2,2)^{*}$ analogue of Seiberg duality.

Our second example of superpotential only involves the adjoint chiral multiplet, and constrains its complexified twisted mass:

$$
W=\operatorname{Tr} X^{l+1}, \quad b^{2}=\mathrm{i} m_{X}=\frac{-1}{l+1}
$$

for some $l \geq 1$. The superpotential constraint sets $b$ to an imaginary value, for which $S_{b}^{4}$ does not make sense. Instead of a surface operator on $S^{2} \subset S_{b}^{4}$ we must thus manipulate the two dimensional theory on $S^{2}$ only. Correspondingly, the matching (2.111) with the Toda CFT is written in the form $Z_{S^{2}}=\left[\langle\cdots\rangle / Z_{S_{b}^{4}}^{\text {free }}\right]_{b^{2}=-1 /(l+1)}$, where the right-hand side is analytically continued after taking the ratio. ${ }^{28}$

For $\mathrm{i} m_{X}=\frac{-1}{l+1}$, the one-loop determinant (2.96) vanishes whenever any $n_{s}>l$ : the numerator factor for $t=s$ and $\mu=n_{s}-l-1$ is $\gamma\left(\mathrm{i} m_{s}-\mathrm{i} m_{s}+\left(n_{s}-l-1-n_{s}\right) \mathrm{i} m_{X}\right)=\gamma(1)=0$. Equivalently, Higgs branch vacua have $X^{n_{s}-1} q_{s} \neq 0$ and are forbidden if $n_{s}>l$ by the F-term equation $X^{l}=0$. The $S^{2}$ partition function in the presence of $W=\operatorname{Tr} X^{l+1}$ is thus a sum over choices of integers $0 \leq n_{s} \leq l$ with $\sum_{s=1}^{N_{f}} n_{s}=N$.

We see that introducing the superpotential $W=\operatorname{Tr} X^{l+1}$ replaces the sum over weights $\sum_{s=1}^{N_{f}} n_{s} h_{s}$ of the symmetric representation $\mathcal{R}\left(N h_{1}\right)$ by a sum over a restricted set of

\footnotetext{
${ }^{28}$ The central charge $c=\left(N_{f}-1\right)\left[1+N_{f}\left(N_{f}+1\right)\left(b^{2}+2+b^{-2}\right)\right]=-\left(N_{f}-1\right)\left(N_{f} l-1\right)\left(N_{f} l+l+1\right) /(l+1)$ is negative for the value $b^{2}=-1 /(l+1)$ we consider.
} 
weights, with $0 \leq n_{s} \leq l$. Those are precisely the weights of the representation with highest weight

$$
\omega_{N, l}=l \omega_{k}+(N-l k) h_{k+1} \quad \text { and Young diagram }
$$

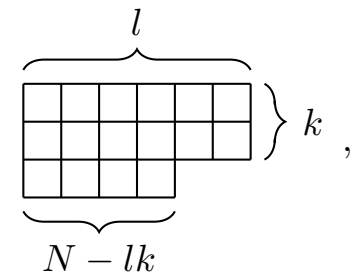

where $k$ is defined by $k l \leq N<(k+1) l$. The "quasi-rectangular" Young diagram is obtained by placing $N$ boxes into as many $l$-box rows as possible followed by a row with any remaining box. For $l \geq N$, none of the one-loop determinants vanish, and the Young diagram is that of the $N$-th symmetric representation: this is the same as for SQCDA. For $l=1$, the Young diagram becomes a column, hence we sum over weights of the $N$-th antisymmetric representation, as for SQCD with no adjoint: correspondingly, the superpotential $W=\operatorname{Tr} X^{2}$ lets us integrate out the adjoint chiral multiplet.

From our experience with SQCD and SQCDA, we expect the sum over weights of $\mathcal{R}\left(\omega_{N, l}\right)$ to have a Toda CFT analogue involving the degenerate operator $\widehat{V}_{-b \omega_{N, l}}$. This is confirmed by the observation that the momenta $-N b h_{1}$ and $-b \omega_{N, l}$ are Weyl conjugate when $b^{2}=\frac{-1}{l+1}$ since

$$
\begin{aligned}
\left\{\frac{1}{b}\left\langle-N b h_{1}-Q, h_{p}\right\rangle \mid 1 \leq p \leq N_{f}\right\} & \\
= & \left\{\frac{N}{N_{f}}+\frac{N_{f}-1}{2} l-N\right\} \cup\left\{\frac{N}{N_{f}}+\frac{N_{f}-1}{2} l-k l \mid 1 \leq k \leq N_{f}-1\right\} \\
& =\left\{\frac{1}{b}\left\langle-b \omega_{N, l}-Q, h_{p}\right\rangle \mid 1 \leq p \leq N_{f}\right\} .
\end{aligned}
$$

Therefore, $\widehat{V}_{-N b h_{1}}$ and $\widehat{V}_{-b \omega_{N, l}}$ are equal up to a scalar factor for this value of $b^{2}$. This assertion should be handled with care, as the Toda CFT is ill defined for $b^{2}<0$.

Trusting the assertion leads us to the proposal ${ }^{29}$

$$
\begin{aligned}
& Z_{S^{2}}^{\mathrm{U}(N) \mathrm{SQCDA}, W}=\operatorname{Tr} X^{l+1}\left(m, \widetilde{m}, m_{X}=\frac{\mathrm{i}}{l+1}, z, \bar{z}\right) \\
&=A|y|^{2 \gamma_{0}}|1-y|^{2 \gamma_{1}}\left[\frac{\left\langle\widehat{V}_{\alpha_{\infty}}(\infty) \widehat{V}_{(\varkappa+N b) h_{1}}(1) \widehat{V}_{-b \omega_{N, l}}(y, \bar{y}) \widehat{V}_{\alpha_{0}}(0)\right\rangle}{\left\langle\widehat{V}_{\alpha_{\infty}}(\infty) \widehat{V}_{\varkappa h_{1}}(1) \widehat{V}_{\alpha_{0}}(0)\right\rangle}\right]_{b^{2} \rightarrow \frac{-1}{l+1}}
\end{aligned}
$$

for some $A$, and with other parameters given below the SQCDA matching (2.88). Importantly, we have moved the $S_{b}^{4}$ partition function of $\mathcal{N}=2$ free hypermultiplets to the right-hand side (in the form of a Toda CFT three-point function), and we only set $b^{2}=\frac{-1}{l+1}$ after evaluating the ratio of Toda CFT correlators. We can thus expect Upsilon functions

\footnotetext{
${ }^{29}$ As explained below (2.10), the factor $A|x|^{2 \gamma_{0}}|1-x|^{2 \gamma_{1}}$ can be absorbed into the partition function. To compare gauge theory and Toda CFT results it is best to keep the factor explicitly.
} 
in the numerator and denominator to cancel, leaving a product of gamma functions which can be analytically continued to $b^{2}=\frac{-1}{l+1}$ and should reproduce one-loop determinants in the left-hand side.

When $l \geq N,(2.111)$ is simply the SQCDA matching (2.88) at i $m_{X}=b^{2}=\frac{-1}{l+1}$, with the same value of $A$. When $l=1$, we expect the claim to reproduce the SQCD result (2.51), and indeed the SQCDA parameters which appear in (2.111) are equal for $\mathrm{i} m_{X}=b^{2}=\frac{-1}{2}$ to the corresponding SQCD parameters, with the exception of $A$.

It is difficult to find $A$ in general, because three-point functions involving $\widehat{V}_{-b \omega_{N, l}}$ take complicated forms for $1<l<N$. Using [58, equations (1.53) and (1.56)], we tested the proposal (2.111) for $N_{f}=N=3$ and $l=2$, which corresponds to the adjoint representation of $\mathrm{SU}(3)$. Three-point functions $\widehat{C}_{-b\left(h_{1}-h_{3}\right), \alpha}^{\alpha-b h}$ associated to non-zero weights $h$ of the adjoint representation are ratios of Gamma functions. When $b^{2}=\frac{-1}{l+1}=\frac{-1}{3}$, they yield the expected one-loop determinants up to a factor $A=b^{9-6 b \varkappa} \gamma(1 / 3)$. For general $b$, the three-point function $\widehat{C}_{-b\left(h_{1}-h_{3}\right), \alpha}^{\alpha}$ associated to the zero weight is expressed in terms of hypergeometric functions evaluated at 1 , but at the point $b^{2}=\frac{-1}{3}$ the value agrees numerically with the Gamma functions expected from gauge theory:

$$
\begin{aligned}
\widehat{C}_{-b\left(h_{1}-h_{N_{f}}\right), Q-\mathrm{i} a}^{Q-\mathrm{i} a}= & \sum_{p=1}^{N_{f}} \prod_{s \neq p}^{N_{f}}\left(\frac{\gamma\left(b\left\langle\mathrm{i} a, h_{p}-h_{s}\right\rangle\right)}{\gamma\left(1+b^{2}+b\left\langle\mathrm{i} a, h_{p}-h_{s}\right\rangle\right)}\right) F\left(\begin{array}{c}
-b^{2}+b\left\langle\mathrm{i} a, h_{s}-h_{p}\right\rangle, 1 \leq s \leq N_{f} \\
1+b\left\langle\mathrm{i} a, h_{s}-h_{p}\right\rangle, s \neq p
\end{array} \mid 1\right)^{2} \\
& \frac{N_{f}=3, b^{2} \rightarrow-1 / 3}{\text { numerically }} \frac{\gamma\left(-b^{2}\right)}{\gamma\left(-2 b^{2}\right)} \prod_{1 \leq s \neq t \leq 3} \gamma\left(b\left\langle\mathrm{i} a, h_{s}-h_{t}\right\rangle-b^{2}\right) .
\end{aligned}
$$

More generally, a Toda CFT four-point function with a fully degenerate vertex operator other than $\widehat{V}_{-b \omega_{N}}$ or $\widehat{V}_{-N b h_{1}}$ (and the usual two generic and one semi-degenerate vertex operators) cannot coincide with the partition function of a surface operator described by a single $\mathcal{N}=(2,2) \mathrm{U}(N)$ vector multiplet coupled to some chiral multiplets, except for special values of $b$ as is the case here. Indeed, as described by Fateev and Litvinov [58], the Toda three-point function $\widehat{C}_{-b \omega, \alpha}^{\alpha-b h}$ only takes the form of a ratio of Gamma functions if the weight $h$ appears with no multiplicity in $\mathcal{R}(\omega)$. Since one-loop determinants are always such ratios, they can only reproduce Toda CFT three-point functions for general $b$ if weights have no multiplicities.

However, higher degenerate fields can be obtained by considering the collision limit of simpler degenerate fields. For instance, the three-point function $\widehat{C}_{-b\left(h_{1}-h_{3}\right), \alpha}^{\alpha}$ mentioned above is equal to a four-point function involving a fundamental and an antifundamental degenerate fields, in the limit where the two punctures collide. In the next section, we match Toda CFT correlators involving more than one (symmetric or antisymmetric) degenerate vertex operator with $S^{2}$ partition functions of quiver gauge theories. Colliding antisymmetric degenerate operators, we obtain expressions for Toda CFT correlators of arbitrary degenerate operators $\widehat{V}_{-b \Omega}$ with two generic and one semi-degenerate vertex operators, for any $b$. 


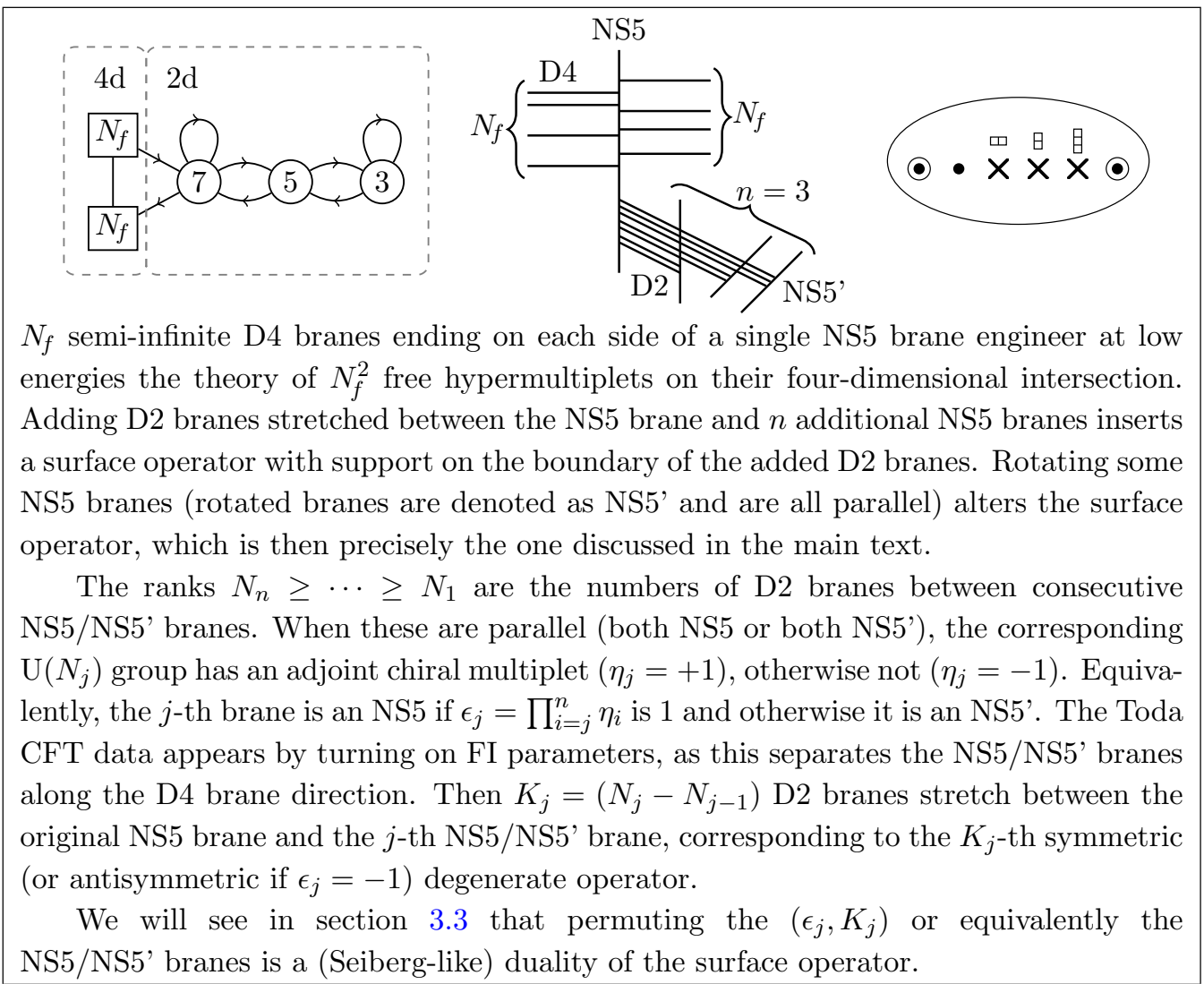

Figure 2. A 4d/2d quiver, its corresponding brane diagram, and Toda CFT correlator.

\subsection{Quivers and multiple Toda degenerates}

We have focused so far on surface operators described by $\mathrm{U}(N)$ gauge theories, which have a single FI parameter. Those correspond to Toda CFT four-point functions, which involve a single anharmonic ratio $x$. Here, we equate the partition function of surface operators described by certain $\mathrm{U}\left(N_{1}\right) \times \cdots \times \mathrm{U}\left(N_{n}\right)$ quiver gauge theories and $(n+3)$-point functions with $n$ symmetric or antisymmetric degenerate operators. In detail, ${ }^{30}$

$$
Z_{S^{2} \subset S_{b}^{4}}^{\prod_{j} \mathrm{U}\left(N_{j}\right), W_{\eta}}(m, z, \bar{z})=A a(x) a(\bar{x})\left\langle\widehat{V}_{\alpha_{\infty}}(\infty) \widehat{V}_{\hat{m}}(1) \prod_{j=1}^{n} \widehat{V}_{-b \Omega\left(K_{j}, \epsilon_{j}\right)}\left(x_{j}, \bar{x}_{j}\right) \widehat{V}_{\alpha_{0}}(0)\right\rangle .
$$

The matching gives a detailed description of the moduli space parametrized by the $z_{j}$. We describe notations below, then consider several limits to fix all parameters of the matching in section 2.4.1. Fine-tuning FI parameters such that degenerate punctures collide on the Toda CFT side, we deduce in section 2.4.2 the microscopic description of the surface operator which corresponds to arbitrary degenerate punctures in the Toda CFT. Brane diagrams (see figure 2) clarify some aspects of the correspondence.

\footnotetext{
${ }^{30}$ Following the arguments below (2.10), the factor $A a(x) a(\bar{x})$ can be absorbed into the partition function. To compare gauge theory and Toda CFT results it is best to keep the factor explicitly.
} 
The surface operator depends on a choice of $n$ signs $\eta_{j}= \pm 1$ and integer parameters $N_{n} \geq \cdots \geq N_{1} \geq 0$. It also depends on $n$ FI and theta parameters combined as

$$
z_{j}=e^{-2 \pi \xi_{j}+\mathrm{i} \vartheta_{j}} \quad \text { and } \quad \hat{z}_{j}=(-1)^{N_{j-1}+N_{j+1}} z_{j}
$$

for $1 \leq j \leq n$, where $N_{0}=0, N_{n+1}=N_{f}$, and the sign is chosen for later convenience. The operator is defined by the $\mathcal{N}=(2,2)$ quiver

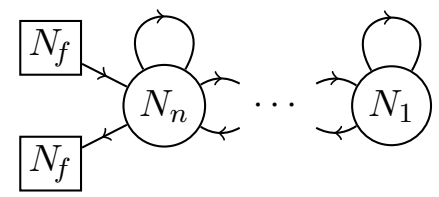

which describes a $\mathrm{U}\left(N_{1}\right) \times \cdots \times \mathrm{U}\left(N_{n}\right)$ vector multiplet coupled to various chiral multiplets. First, $N_{f}$ fundamentals $q_{t}$ and $N_{f}$ antifundamentals $\widetilde{q}_{t}$ of $\mathrm{U}\left(N_{n}\right)$. Next, for each $1 \leq$ $j \leq n-1$, one pair of bifundamentals of $\mathrm{U}\left(N_{j}\right) \times \mathrm{U}\left(N_{j+1}\right): \phi_{j(j+1)}$ in the representation $N_{j} \otimes \bar{N}_{j+1}$ and $\phi_{(j+1) j}$ in the representation $\bar{N}_{j} \otimes N_{j+1}$. Finally, for each $1 \leq j \leq n$, one adjoint $X_{j}$. The (complexified) twisted masses $m_{t}, \widetilde{m}_{t}, m_{j(j+1)}, m_{(j+1) j}$ and $m_{j j}$ of these fields are constrained by a superpotential coupling $W_{\eta}$.

The superpotential has the following terms,

$$
\begin{cases}\operatorname{Tr}\left(X_{j}^{2}\right) & \text { for } 1 \leq j \leq n \text { if } \eta_{j}=-1, \\ \operatorname{Tr}\left(\phi_{j(j+1)} \phi_{(j+1) j} \phi_{j(j-1)} \phi_{(j-1) j}\right) & \text { for } 1<j<n \text { if } \eta_{j}=-1, \\ \operatorname{Tr}\left(X_{j} \phi_{j(j+1)} \phi_{(j+1) j}\right) & \text { for } 1 \leq j<n \text { if } \eta_{j}=1, \\ \operatorname{Tr}\left(X_{j} \phi_{j(j-1)} \phi_{(j-1) j}\right) & \text { for } 1<j \leq n \text { if } \eta_{j}=1 .\end{cases}
$$

In other words the adjoint multiplets of nodes with $\eta_{j}=1$ have a cubic coupling to neighboring bifundamental multiplets, while nodes with $\eta_{j}=-1$ entail a quartic coupling of neighboring bifundamental multiplets. The $\operatorname{Tr}\left(X_{j}^{2}\right)$ term for $\eta_{j}=-1$ gives a mass to the adjoint multiplet $X_{j}$, hence the theory (2.116) is equivalent in the low-energy to the analogous theory (1.8) from the introduction, which omits these $X_{j}$. Here, we include adjoint multiplets for all nodes to simplify signs in the definition $(2.115)$ of $\hat{z}_{j}$. Indeed, integrating out $X_{j}$ when $\eta_{j}=-1$ shifts the corresponding theta angle $z_{j} \rightarrow(-1)^{N_{j}-1} z_{j}$, hence in order to keep $\hat{z}_{j}$ fixed one should complicate the definition (2.115) of $\hat{z}_{j}$ by including the sign $(-1)^{N_{j}-1}$.

The superpotential $W_{\eta}$ must have $R$-charge 2 (twisted mass i) to be supersymmetric. This fixes twisted masses of bifundamental and adjoint multiplets in terms of the signs $\eta$ and a single continuous parameter, ${ }^{31}$ which will match with $b^{2}$ in the Toda CFT. To ease the comparison with the Toda CFT correlator, we define signs $\epsilon_{j}=\prod_{i=j}^{n} \eta_{i}$ for $1 \leq j \leq n+1$

\footnotetext{
${ }^{31}$ The full flavour symmetry of the two dimensional theory is $S\left[\mathrm{U}\left(N_{f}\right) \times \mathrm{U}\left(N_{f}\right)\right] \times \mathrm{U}(1)$, where the first factor acts on fundamental and antifundamental chiral multiplets. Under the $\mathrm{U}(1)$ factor, the adjoint chiral multiplet $X_{j}$ has charge $\epsilon_{j}+\epsilon_{j+1}$ and the bifundamental multiplets $\phi_{(j-1) j}$ and $\phi_{j(j-1)}$ have charge $-\epsilon_{j}$, where $\epsilon_{j}=\prod_{i=j}^{n} \eta_{i}$.
} 
and find

$$
\begin{array}{r}
\qquad m_{j j}= \begin{cases}-1-b^{2} & \text { if } \epsilon_{j+1}=\epsilon_{j}=-1, \\
-1 / 2 & \text { if } \epsilon_{j+1} \neq \epsilon_{j}, \\
b^{2} & \text { if } \epsilon_{j+1}=\epsilon_{j}=+1,\end{cases} \\
\mathrm{i} m_{(j-1) j}+\mathrm{i} m_{j(j-1)}= \begin{cases}b^{2} & \text { if } \epsilon_{j}=-1, \\
-1-b^{2} & \text { if } \epsilon_{j}=+1 .\end{cases}
\end{array}
$$

Equivalently, $W_{\eta}$ could be defined as containing all gauge invariant combinations of the fields which have total $R$-charge 2 (twisted mass i), given the mass assignment (2.118). As always, the twisted masses and $R$-charges of fundamental and antifundamental chiral multiplets are unconstrained.

On the other hand, the Toda CFT $(n+3)$-point function involves two generic and one semi-degenerate vertex operators $\widehat{V}_{\alpha_{\infty}}(\infty), \widehat{V}_{\hat{m}}(1)$, and $\widehat{V}_{\alpha_{0}}(0)$ with momenta

$$
\begin{aligned}
\alpha_{0}=Q-\frac{1}{b} \sum_{s=1}^{N_{f}} \mathrm{i} m_{s} h_{s}, & \hat{m}=\left(\varkappa+N_{n} b\right) h_{1}, \\
\alpha_{\infty}=Q-\frac{1}{b} \sum_{s=1}^{N_{f}} \mathrm{i} \widetilde{m}_{s} h_{s}, & \varkappa=\frac{1}{b} \sum_{s=1}^{N_{f}}\left(1+\mathrm{i} m_{s}+\mathrm{i} \widetilde{m}_{s}\right),
\end{aligned}
$$

which coincide with those of earlier sections. It also involves $n$ fully degenerate vertex operators $\widehat{V}_{-b \Omega\left(K_{j}, \epsilon_{j}\right)}\left(x_{j}, \bar{x}_{j}\right)$ at

$$
x_{j}=\prod_{i=j}^{n} \hat{z}_{i} \quad \text { for } 1 \leq j \leq n .
$$

Each degenerate operator is labeled by the highest weight $\Omega(K,+1)=K h_{1}$ of a symmetric representation or $\Omega(K,-1)=\omega_{K}$ of an antisymmetric representation of $A_{N_{f}-1}$, depending on the signs $\epsilon_{j}=\prod_{i=j}^{n} \eta_{i}$ and the integers

$$
K_{1}=N_{1}, \quad \text { and } \quad K_{j}=N_{j}-N_{j-1} \quad \text { for } 1<j \leq n .
$$

Finally, the factors $A$ and $a$ are

$$
\begin{aligned}
A & =b^{N_{n} N_{f}\left(1+b^{2}\right)-N_{n}^{2} b^{2}-2 N_{n} b \varkappa} \prod_{j \mid \epsilon_{j}=+1} \prod_{1 \leq \nu \leq K_{j}} \gamma\left(-\nu b^{2}\right), \\
a(x) a(\bar{x}) & =\prod_{j=1}^{n}\left|x_{j}\right|^{2 \beta_{j}} \prod_{j=1}^{n}\left|1-x_{j}\right|^{2 \gamma_{j}} \prod_{i<j}^{n}\left|x_{j}-x_{i}\right|^{2 \gamma_{i j}},
\end{aligned}
$$

with the exponents

$$
\begin{aligned}
\beta_{j} & =\Delta\left(-b \Omega\left(K_{j}, \epsilon_{j}\right)\right)-\frac{K_{j}}{N_{f}} \sum_{t=1}^{N_{f}} \mathrm{i} m_{t}+\frac{K_{j}\left(N_{f}-K_{j}\right)}{2 N_{f}} b^{2}-N_{j-1} \mathrm{i} m_{j(j-1)}-K_{j} \sum_{i=j+1}^{n} \mathrm{i} m_{(i-1) i}, \\
\gamma_{j} & =\left(-1-b^{2}\right) K_{j}+b\left(\varkappa+N_{n} b\right) K_{j} / N_{f}, \\
\gamma_{i j} & = \begin{cases}b^{2} K_{i}-b^{2} K_{i} K_{j} / N_{f} & \text { if } \epsilon_{j}=-1, \\
\left(-1-b^{2}\right) K_{i}-b^{2} K_{i} K_{j} / N_{f} & \text { if } \epsilon_{j}=+1,\end{cases}
\end{aligned}
$$


for $1 \leq i<j \leq n$. When $n=1$, the matching (2.114) reproduces the known cases of $\operatorname{SQCD}\left(\eta_{1}=-1\right)$ and SQCD with an adjoint $\left(\eta_{1}=1\right)$. Also, for $n>1$ setting $N_{1}=0$ reduces the matching to the case $n \rightarrow n-1$.

As a preliminary check of the equality (2.114), we can recognize a few symmetries. Permuting the flavours of fundamental quarks $q_{t}$, hence their twisted masses $m_{t}$, does not alter the partition function. This is translated on the Toda CFT side into a Weyl transformation of the momentum $\alpha_{0}$, which permutes the $\left\langle\alpha_{0}-Q, h_{t}\right\rangle$. Similarly, permuting the $\widetilde{m}_{t}$ amounts to a Weyl transformation of $\alpha_{\infty}$. Next, performing charge conjugation on all gauge group factors maps $\hat{z}_{j} \rightarrow \hat{z}_{j}^{-1}, m_{t} \leftrightarrow \widetilde{m}_{t}$, and $m_{j(j+1)} \leftrightarrow m_{(j+1) j}$ : this corresponds on the Toda CFT side to the conformal map $x \rightarrow x^{-1}$, which swaps $\alpha_{0} \leftrightarrow \alpha_{\infty}$ and maps $x_{j} \rightarrow x_{j}^{-1}$. The transformation of $a(x) a(\bar{x})$ compensates exactly the conformal factor $\left|x_{j}\right|^{-4 \Delta\left(-b \Omega\left(K_{j}, \epsilon_{j}\right)\right)}$ for each $j$. Finally, shifting the twisted masses of bifundamentals while keeping the sums $m_{j(j+1)}+m_{(j+1) j}$ constant amounts to a constant gauge transformation, whose sole effect on the partition function is in overall powers of $\left|x_{j}\right|^{2}$ : on the Toda CFT side of (2.114), only the exponents $\beta_{j}$ change.

\subsubsection{Matching parameters for quivers}

We first expand the partition function and the correlator in the s-channel, that is, the region where $0<\left|x_{1}\right|<\cdots<\left|x_{n}\right|<1$ or equivalently where all FI parameters are positive: $\left|\hat{z}_{j}\right|<1$. We map vacua of the gauge theory to choices of internal momenta in the correlator. The classical and one-loop contributions match as expected with the exponents and three-point functions, while the vortex partition functions give predictions for Toda CFT conformal blocks (see appendix A.5). This check fixes $\left\{K_{j}, \epsilon_{j}\right\}$, the momentum $\alpha_{0}$, the overall constant factor $A$ and the exponents $\beta_{j}+\sum_{i<j} \gamma_{i j}$. The momentum $\alpha_{\infty}$ is fixed by the symmetry under charge conjugation discussed earlier. Then, we justify the relation between the gauge theory data $\left\{\eta_{j}, \hat{z}_{j}\right\}$ and the Toda CFT data $\left\{\epsilon_{j}, x_{j}\right\}$ by counting distinct exponents in the limit where two neighboring punctures collide. Comparing the exponents only fixes the momentum $\hat{m}$ and the exponents $\gamma_{n}$ and $\gamma_{(j-1) j}$. The remaining exponents $\gamma_{j}$ and $\gamma_{i j}$ are fixed thanks to Seiberg dualities which translates in this setting to permutations of the $n$ punctures (see section 3.3).

It is easiest to find Higgs branch vacua of the gauge theory by solving the D-term and F-term equations, assuming as before that fundamental chiral multiplets have generic twisted masses $m_{s}$. The derivation goes as follows. Diagonalize all $\sigma_{j}$. Introduce $\mathrm{i} \sigma_{n+1}=$ $\operatorname{diag}\left(-\mathrm{i} m_{1}, \ldots,-\mathrm{i} m_{N_{f}}\right), N_{n+1}=N_{f}$, and $N_{0}=0$ to simplify the discussion. Integrate out all $X_{j}$ which have twisted mass $m_{j j}=\mathrm{i} / 2$, that is, $\eta_{j}=-1$. The D-term equations (for $\left.\left|\hat{z}_{j}\right|<1\right)$ impose that the images of $X_{j}, \phi_{j(j+1)}$ and $\phi_{j(j-1)}$ span $\mathbb{C}^{N_{j}}$, hence all eigenvalues of $\sigma_{j}$ are constrained to be equal to another eigenvalue of $\sigma_{j}$ or of $\sigma_{j \pm 1}$, minus a twisted mass. All eigenvalues of $\mathrm{i} \sigma_{j}$ thus take the form $\mathrm{i} \sigma_{j, \mathrm{a}}=-\mathrm{i} m_{s}-\sum_{i=j+1}^{n} \mathrm{i} m_{(i-1) i}+\mu\left(1+b^{2}\right)-$ $\nu b^{2}$ where $\mu, \nu \in \mathbb{Z}_{\geq 0}$. Using the F-term constraint, one can then bound the multiplicity of such an eigenvalue by the multiplicity of the eigenvalue $\mathrm{i} \sigma_{j, \mathrm{a}}-\mathrm{i} m_{j k}$ of $\mathrm{i} \sigma_{k}$, for $k \in\{j, j \pm 1\}$ (only $k \in\{j \pm 1\}$ if $X_{j}$ was integrated out). Since each eigenvalue $-\mathrm{i} m_{s}$ of $\mathrm{i} \sigma_{n+1}$ has multiplicity 1 , we deduce by induction on $n+1-j, \mu$, and $\nu$ that all eigenvalues have multiplicity 1 . The statement is in fact stronger: for any eigenvalue $\mathrm{i} \sigma_{j, \mathrm{a}}$ of $\mathrm{i} \sigma_{j}$, and for 
$k \in\{j, j \pm 1\}$ (or only $k \in\{j \pm 1\}$ ), $\mathrm{i} \sigma_{j, \mathrm{a}}-\mathrm{i} m_{j k}$ is an eigenvalue of $\mathrm{i} \sigma_{k}$, and the relevant component of $\phi_{j k}$ is non-zero. Solving the F-term constraints is then a combinatorical problem whose details depend on the superpotential $W_{\eta}$.

At the end of the day, one finds that vacua obey

$$
\mathrm{i} \sigma_{j}=\operatorname{diag}\left(-\mathrm{i} m_{s}-\sum_{i=j+1}^{n}\left(\mathrm{i} m_{(i-1) i}\right)-\nu b^{2} \mid 0 \leq \nu<n_{s}^{j}, 1 \leq s \leq N_{f}\right)
$$

for $1 \leq j \leq n$, where $n_{s}^{j} \geq 0$ are integers such that $\sum_{s=1}^{N_{f}} n_{s}^{j}=N_{j}$ and

$$
\begin{cases}n_{s}^{j-1} \leq n_{s}^{j} \leq n_{s}^{j-1}+1 & \text { if } \epsilon_{j}=-1 \\ n_{s}^{j-1} \leq n_{s}^{j} & \text { if } \epsilon_{j}=+1\end{cases}
$$

where $n_{s}^{0}=0$. These conditions are equivalent to requiring that for each $1 \leq j \leq n$ the difference $h_{\left[n^{j}\right]}-h_{\left[n^{j-1}\right]}=\sum_{s=1}^{N_{f}}\left(n_{s}^{j}-n_{s}^{j-1}\right) h_{s}$ is a weight of the symmetric or antisymmetric representation $\mathcal{R}\left(\Omega\left(K_{j}, \epsilon_{j}\right)\right)$ of $\operatorname{rank} K_{j}=N_{j}-N_{j-1}$. The $S^{2}$ partition function is then a sum

$$
Z_{S^{2}}=\sum_{\left\{n_{s}^{j}\right\}} Z_{\mathrm{cl}} Z_{11} Z_{\mathrm{v}} Z_{\overline{\mathrm{v}}}
$$

over choices of $\left\{n_{s}^{j}\right\}$ consistent with the constraints above. Terms of this sum are in a natural bijection with terms of the s-channel decomposition of the Toda CFT correlator in (2.114): the internal momenta are $\alpha_{0}-b h_{\left[n^{j}\right]}$ for $1 \leq j \leq n$. Thus, counting terms fixes the degenerate momenta $-b \Omega\left(K_{j}, \epsilon_{j}\right)$ in terms of the $N_{j}$ and $\eta_{j}$.

Since the Higgs branch and Coulomb branch representations of $S^{2}$ partition functions coincide, $Z_{\mathrm{cl}} Z_{11}$ is the residue at the pole (2.127) of the Coulomb branch integrand, and $Z_{\mathrm{v}} Z_{\overline{\mathrm{v}}}$ is the additional contribution from poles for which $\mathrm{i} \sigma_{j}^{ \pm}$is $(2.127)$ plus integers. We find in particular that the classical contribution reproduces the powers of $x_{j}$ expected from the Toda CFT up to shifts by $\beta_{j}+\sum_{i=1}^{j-1} \gamma_{i j}$,

$$
\begin{aligned}
Z_{\mathrm{cl}} & =\prod_{j=1}^{n}\left|z_{j}\right|^{2 \operatorname{Tr} \mathrm{i} \sigma_{j}}=\prod_{j=1}^{n}\left|z_{j}\right|^{2\left[-\sum_{s=1}^{N_{f}}\left(n_{s}^{j} \mathrm{i} m_{s}\right)-N_{j} \sum_{i=j+1}^{n}\left(\mathrm{i} m_{(i-1) i}\right)-\sum_{s=1}^{N_{f}} \sum_{\nu=0}^{n_{s}^{j}-1} \nu b^{2}\right]} \\
& =\prod_{j=1}^{n}\left|x_{j}\right|^{2\left[\beta_{j}+\sum_{i=1}^{j-1}\left(\gamma_{i j}\right)+\Delta\left(\alpha_{0}-b h_{\left[n_{j}\right]}\right)-\Delta\left(\alpha_{0}-b h_{\left[n^{j}-1\right]}\right)-\Delta\left(-b \Omega\left(K_{j}, \epsilon_{j}\right)\right)\right]}
\end{aligned}
$$

provided that $\alpha_{0}$ is as given in (2.119), and $\beta_{j}+\sum_{i=1}^{j-1} \gamma_{i j}$ as in (2.124) and (2.126). By symmetry, $\alpha_{\infty}$ is as given in (2.119). Similarly, a tedious calculation shows that for each term the one-loop determinant $Z_{11}$ matches with the product of Toda CFT three-point functions, up to precisely the constant $A$ given in (2.122).

Next, let us probe the collision of two neighboring punctures, starting again from the schannel $0<\left|x_{1}\right|<\cdots<\left|x_{n}\right|<1$. The Coulomb branch representation of the $S^{2}$ partition function of interest has the form

$$
Z_{S^{2}}^{\prod_{j} \mathrm{U}\left(N_{j}\right), W_{\eta}}=\prod_{j=1}^{n}\left[\frac{1}{N_{j} !} \sum_{B_{j} \in \mathbb{Z}^{N_{j}}} \int \frac{\mathrm{d}^{N_{j}} \sigma_{j}}{(2 \pi)^{N_{j}}}\right] \prod_{j=1}^{n}\left[z_{j}^{\operatorname{Tr} \mathrm{i} \sigma_{j}^{+}} \bar{z}_{j}^{\operatorname{Tr} \mathrm{i} \sigma_{j}^{-}}\right] Z_{11, \mathrm{v} . \mathrm{m} .} Z_{11, \mathrm{c} . \mathrm{m} .}
$$


where $\mathrm{i} \sigma_{j}^{ \pm}=\mathrm{i} \sigma_{j} \pm B_{j} / 2, Z_{11, \mathrm{v} . \mathrm{m} .}$ is the one-loop determinant of vector multiplets (a Vandermonde factor and a sign), and $Z_{11, c . m}$. is the one-loop determinant of chiral multiplets (a product of Gamma functions). Collecting all factors which depend on $\sigma_{k}^{ \pm}$for a given $k<n$ yields the integral

$$
\begin{aligned}
& Z_{k}= \sum_{B_{k} \in \mathbb{Z}^{N_{k}}} \int \frac{\mathrm{d}^{N_{k}} \sigma_{k}}{N_{k} !(2 \pi)^{N_{k}}}\left[(-1)^{N_{k}-1} z_{k}\right]^{\operatorname{Tr} \sigma_{k}^{+}}\left[(-1)^{N_{k}-1} \bar{z}_{k}\right]^{\operatorname{Tr} \sigma_{k}^{-}} \prod_{i<j}^{N_{k}} \prod_{ \pm}\left(\sigma_{k i}^{ \pm}-\sigma_{k j}^{ \pm}\right) \\
& \cdot \prod_{i=1}^{N_{k}}\left[\prod_{j=1}^{N_{k}} \frac{\Gamma\left(-\mathrm{i} m_{k k}-\mathrm{i} \sigma_{k i}^{+}+\mathrm{i} \sigma_{k j}^{+}\right)}{\Gamma\left(1+\mathrm{i} m_{k k}+\mathrm{i} \sigma_{k i}^{-}-\mathrm{i} \sigma_{k j}^{-}\right)}\right. \\
&\left.\cdot \prod_{\substack{l \in\{k \pm 1\} \\
1 \leq j \leq N_{l}}}\left[\frac{\Gamma\left(-\mathrm{i} m_{k l}+\mathrm{i} \sigma_{l j}^{+}-\mathrm{i} \sigma_{k i}^{+}\right)}{\Gamma\left(1+\mathrm{i} m_{k l}-\mathrm{i} \sigma_{l j}^{-}+\mathrm{i} \sigma_{k i}^{-}\right)} \frac{\Gamma\left(-\mathrm{i} m_{l k}-\mathrm{i} \sigma_{l j}^{+}+\mathrm{i} \sigma_{k i}^{+}\right)}{\Gamma\left(1+\mathrm{i} m_{l k}+\mathrm{i} \sigma_{l j}^{-}-\mathrm{i} \sigma_{k i}^{-}\right)}\right]\right]
\end{aligned}
$$

which resembles the $S^{2}$ partition function of SQCDA with $N_{k}$ colors and $N_{k-1}+N_{k+1}$ flavours, with twisted masses $m_{k l}-\sigma_{l j}$ and $m_{l k}+\sigma_{l j}$. The shifts of $\sigma_{l j}$ by $\pm B_{l j} / 2$ cannot be incorporated in such twisted masses, as the ratios of Gamma functions involve both $\sigma_{l j}^{+}$and $\sigma_{l j}^{-}$.

However, we can still apply the same techniques as in section 2.3, and close the $\mathrm{i} \sigma_{k}$ integration contours towards $\pm \infty$ depending on whether $\left|z_{k}\right| \lessgtr 1$. The sum over poles factorizes as in the case of SQCDA, and the resulting vortex and antivortex partition functions are those of SQCDA with twisted masses $m_{k l}-\sigma_{l j}^{+}$and $m_{l k}+\sigma_{l j}^{+}$for vortices, and $m_{k l}-\sigma_{l j}^{-}$and $m_{l k}+\sigma_{l j}^{-}$for antivortices. As we saw in section 2.3, those vortex partition functions have branch points when $\hat{z}_{k}=(-1)^{N_{k-1}+N_{k+1}} z_{k}$ is 1 or $\infty$. We now prove that the powers of $1-\hat{z}_{k}$ which appear in an expansion of $Z_{\mathrm{v}}$ near $\hat{z}_{k}=1$ coincide with the powers of $x_{k+1}-x_{k}$ obtained in the fusion of the punctures at $x_{k}$ and $x_{k+1}$. This implies that $x_{k}=x_{k+1} \hat{z}_{k}$, as announced, and fixes $\gamma_{k(k+1)}$. To proceed further, we need to distinguish the cases $\eta_{k}= \pm 1$.

If $\eta_{k}=-1$, then $\mathrm{i} m_{k k}=-1 / 2$, and the adjoint chiral multiplet one-loop determinant is simply a sign. Thus, the vortex partition functions are those of SQCD. From (2.69), the exponents of $1-\hat{z}_{k}$ which occur in an expansion near 1 are 0 and

$$
\begin{aligned}
N_{k-1}\left(1+\mathrm{i} m_{k(k-1)}+\mathrm{i} m_{(k-1) k}\right)+N_{k+1}\left(1+\mathrm{i} m_{k(k+1)}+\mathrm{i} m_{(k+1) k}\right)-N_{k} \\
= \begin{cases}-K_{k}\left(1+b^{2}\right)-K_{k+1} b^{2} & \text { if } \epsilon_{k}=-\epsilon_{k+1}=-1, \\
K_{k} b^{2}+K_{k+1}\left(1+b^{2}\right) & \text { if } \epsilon_{k}=-\epsilon_{k+1}=+1 .\end{cases}
\end{aligned}
$$

The analogous limit in the Toda CFT correlator is $x_{k} \rightarrow x_{k+1}$. The channel where the punctures at $x_{k}$ and $x_{k+1}$ are fused allows two internal momenta. Indeed, $\epsilon_{k}=-\epsilon_{k+1}$, hence one of the punctures is labeled by a symmetric representation and the other one by an antisymmetric representation. The fusion of two such representations is the direct sum of two irreducible representations:

$$
\mathcal{R}\left(K h_{1}\right) \otimes \mathcal{R}\left(\omega_{L}\right)=\mathcal{R}\left(K h_{1}+\omega_{L}\right) \oplus \mathcal{R}\left((K-1) h_{1}+\omega_{L+1}\right)
$$


assuming $K, L \geq 1$. The corresponding exponents of $x_{k+1}-x_{k}$ are

$$
\begin{gathered}
\Delta\left(-K b h_{1}-b \omega_{L}\right)-\Delta\left(-K b h_{1}\right)-\Delta\left(-b \omega_{L}\right)=-K b^{2}+\frac{K L}{N_{f}} b^{2}, \\
\Delta\left(-(K-1) b h_{1}-b \omega_{L+1}\right)-\Delta\left(-K b h_{1}\right)-\Delta\left(-b \omega_{L}\right)=L\left(1+b^{2}\right)+\frac{K L}{N_{f}} b^{2},
\end{gathered}
$$

and match with the gauge theory exponents up to precisely $\gamma_{k(k+1)}$ given in (2.126). Indeed, if $\epsilon_{k}=-\epsilon_{k+1}=-1$, then $K$ and $L$ above are $K_{k+1}$ and $K_{k}$, the first Toda CFT exponent corresponds to the gauge theory exponent (2.133), and the second to 0 . If $\epsilon_{k}=-\epsilon_{k+1}=1$, then $K=K_{k}$ and $L=K_{k+1}$, the first Toda CFT exponent corresponds to 0 and the second to $(2.133)$.

If instead $\eta_{k}=+1$, then the adjoint chiral multiplet remains, and the vortex partition functions involve more powers of $1-\hat{z}_{k}$, given in (2.100). Namely,

$$
\begin{aligned}
\left(1-\hat{z}_{k}\right)^{-\nu+\nu N_{k-1}\left(1+\mathrm{i} m_{k(k-1)}+\mathrm{i} m_{(k-1) k}\right)+\nu N_{k+1}\left(1+\mathrm{i} m_{k(k+1)}+\mathrm{i} m_{(k+1) k}\right)+\nu\left[2 N_{k}-\nu-1\right] \mathrm{i} m_{k k}} \\
=\left(1-\hat{z}_{k}\right)^{-\nu\left(1+\mathrm{i} m_{k k}\right)+\nu\left[K_{k}-K_{k+1}-\nu\right] \mathrm{i} m_{k k}}
\end{aligned}
$$

for $0 \leq \nu \leq N_{k}$. The remaining $\sigma_{j}$ integrals $(j \neq k)$ do not affect these exponents. From the derivation of (2.100), we know that the contribution for a given $\nu$ comes from the region where $\nu$ components $\sigma_{k, \text { a }}$ of $\sigma_{k}$ are large. The corresponding Gamma functions in the Coulomb branch integral are expanded as powers of $\mathrm{i} \sigma_{k, \mathrm{a}}^{ \pm}$. Afterwards, one can close contours of all $\sigma_{j}$ for $j<k$ as we have done to find the s-channel expansion. The Gamma functions which were expanded in powers of $\mathrm{i} \sigma_{k, \mathrm{a}}^{ \pm}$do not contribute poles, hence the sum over poles is non-empty only if $N_{k}-\nu \geq N_{k-1} \geq \cdots \geq N_{1}$. As a result, $\nu \leq N_{k}-N_{k-1}=K_{k}$. Changing variables to $\mu=K_{k}-\nu$, we deduce

$$
Z=\left|1-\hat{z}_{k}\right|^{2\left[-K_{k}\left(1+\mathrm{i} m_{k k}\right)\right]} \sum_{\mu=0}^{K_{k}}\left|1-\hat{z}_{k}\right|^{2\left[\mu\left(1+\mathrm{i} m_{k k}\right)-\left(K_{k}-\mu\right)\left(K_{k+1}-\mu\right) \mathrm{i} m_{k k}\right]}(\text { series })
$$

where (series) denote series in non-negative integer powers of $1-\hat{z}_{k}$ and $\overline{1-\hat{z}_{k}}$. In section 3.3, we relate the $S^{2}$ partition function of the quiver gauge theory we are studying to another such partition function, with in particular $K_{k} \leftrightarrow K_{k+1}$. This restricts the sum over $\mu$ to $0 \leq \mu \leq \min \left(K_{k}, K_{k+1}\right)$. On the Toda CFT side, the limit is $x_{k} \rightarrow x_{k+1}$, and we are interested in the fusion of two degenerate punctures, labeled by two symmetric or two antisymmetric representations since $\epsilon_{k}=\epsilon_{k+1}$. Given that

$$
\begin{aligned}
\mathcal{R}\left(\omega_{K}\right) \otimes \mathcal{R}\left(\omega_{L}\right) & =\bigoplus_{\mu=0}^{\min (K, L)} \mathcal{R}\left(\omega_{K+L-\mu}+\omega_{\mu}\right), \\
\mathcal{R}\left(K h_{1}\right) \otimes \mathcal{R}\left(L h_{1}\right) & =\bigoplus_{\mu=0}^{\min (K, L)} \mathcal{R}\left((K+L-\mu) h_{1}+\mu h_{2}\right),
\end{aligned}
$$


the Toda CFT exponents of $x_{k+1}-x_{k}$ are

$$
\begin{aligned}
& \Delta\left(-b \omega_{K+L-\mu}-b \omega_{\mu}\right)-\Delta\left(-b \omega_{K}\right)-\Delta\left(-b \omega_{L}\right) \\
& \quad=\frac{K L}{N_{f}} b^{2}-\mu b^{2}+(K-\mu)(L-\mu)\left(b^{2}+1\right) \quad \text { if } \epsilon_{k}=\epsilon_{k+1}=-1, \\
& \Delta\left(-(K+L-\mu) b h_{1}-\mu b h_{2}\right)-\Delta\left(-K b h_{1}\right)-\Delta\left(-L b h_{1}\right) \\
& \quad=\frac{K L}{N_{f}} b^{2}+\mu\left(b^{2}+1\right)-(K-\mu)(L-\mu) b^{2} \quad \text { if } \epsilon_{k}=\epsilon_{k+1}=+1,
\end{aligned}
$$

where $K$ and $L$ are $K_{k}$ and $K_{k+1}$. Again, the Toda CFT exponents match with the gauge theory exponents up to precisely $\gamma_{k(k+1)}$ given in (2.126).

Note that matching the number of distinct powers of $1-\hat{z}_{k}$ in gauge theory with the number of internal momenta in the fusion of punctures at $x_{k-1}$ and $x_{k}$ is enough to fix the relation between the signs $\left\{\eta_{j}\right\}$ and $\left\{\epsilon_{j}\right\}$. When the adjoint $X_{j}$ can be integrated out $\left(\eta_{j}=-1\right)$, the gauge theory involves two exponents only, and correspondingly the two neighboring punctures are labeled by different types of representations (one is symmetric and the other antisymmetric), whose fusion has two terms. When the adjoint $X_{j}$ remains $\left(\eta_{j}=+1\right)$, the gauge theory involves many exponents, and the two punctures have the same type, hence a fusion with many terms.

The situation is very similar in the limit $x_{n}=\hat{z}_{n} \rightarrow 1$. The gauge theory involves two exponents if $\eta_{n}=-1$, and $N_{n}-N_{n-1}$ if $\eta_{n}=+1$. On the Toda CFT side, the fusion of the semidegenerate momentum $\hat{m}$ with the degenerate $-b \Omega\left(K_{n}, \epsilon_{n}\right)$ gives two momenta if $\epsilon_{n}=-1$, and $K_{n}$ if $\epsilon_{n}=+1$. Hence $\epsilon_{n}=\eta_{n}$ and $K_{n}=N_{n}-N_{n-1}$. Calculating the exponents and comparing them fixes $\hat{m}$ to (2.119) and $\gamma_{n}$ to (2.125).

All other exponents $\gamma_{i j}$ and $\gamma_{j}$ are fixed thanks to the identification of permutations of degenerate punctures with gauge theory dualities found in section 3.3.1.

\subsubsection{Arbitrary Toda degenerates}

We now consider the matching (2.114) in the case where $K_{j+1} \geq K_{j}$ for $1 \leq j \leq n-1$, and $\epsilon_{j}=-1$ for all $1 \leq j \leq n$, that is, $\eta_{n}=-1$ and $\eta_{j}=+1$ for all $1 \leq j \leq n-1$. In the course of fixing parameters for the matching, we have found that the expansion near $x_{k}=x_{k+1}$ involves the $\min \left(K_{k}, K_{k+1}\right)=K_{k}$ powers (2.138) of $x_{k+1}-x_{k}=x_{k+1}\left(1-\hat{z}_{k}\right)$, for $1 \leq k \leq n-1$. Given our assumptions, these exponents all have a non-negative real part (the vortex partition functions contribute integer exponents $\nu \geq 0$ ):

$$
\operatorname{Re}\left(\left(K_{k}-\mu\right) b^{2}+\left(K_{k}-\mu\right)\left(K_{k+1}-\mu\right)\left(1+b^{2}\right)+\nu\right) \geq 0 .
$$

The real part vanishes if and only if $\mu=K_{k}$ and $\nu=0$. As $\hat{z}_{k} \rightarrow 1$, only the term with $\mu=K_{k}$ and $\nu=0$ remains. On the Toda CFT side, this limit selects the fusion

$$
\mathcal{R}\left(\omega_{K_{k+1}}\right) \otimes \mathcal{R}\left(\omega_{K_{k}}\right) \longrightarrow \mathcal{R}\left(\omega_{K_{k+1}}+\omega_{K_{k}}\right)
$$

We can carry this process further and take the fusion of arbitrarily many antisymmetric degenerate operators. For definiteness, let us send $x_{k} \rightarrow x_{n}$ for $k$ going from $n-1$ to 1 , in 
this order. At a given step $x_{k} \rightarrow x_{n}$, the Littlewood-Richardson rule gives

$$
\mathcal{R}(\Omega) \otimes \mathcal{R}\left(\omega_{K_{k}}\right)=\bigoplus_{h \in \mathcal{R}\left(\omega_{K_{k}}\right)}^{\prime} \mathcal{R}(\Omega+h)
$$

with a sum running over weights $h$ of $\mathcal{R}\left(\omega_{K_{k}}\right)$ such that $\Omega+h$ is a dominant weight. In our setting, $\Omega=\omega_{K_{n}}+\cdots+\omega_{K_{k+1}}$. The power of $x_{n}-x_{k}$ for a weight $h$ is

$$
\Delta(-b \Omega-b h)-\Delta(-b \Omega)-\Delta\left(-b \omega_{K_{k}}\right)+\sum_{l=k+1}^{n} \gamma_{k l}=b\left\langle Q, \omega_{K_{k}}-h\right\rangle+b^{2}\left\langle\Omega, \omega_{K_{k}}-h\right\rangle,
$$

which has a positive real part unless $h=\omega_{K_{k}}$, in which case it vanishes. Thus, setting $x_{k}=x_{n}$ selects precisely the fusion of $-b \Omega$ and $-b \omega_{K_{k}}$ into $-b \Omega-b \omega_{K_{k}}$.

Any dominant weight $\Omega$ is a sum of fundamental weights, hence the four-point function of two generic and one semi-degenerate vertex operators with an arbitrary degenerate vertex operator $\widehat{V}_{-b \Omega}$ is equal to the partition function of an $S^{2}$ surface operator built from a certain quiver on $S_{b}^{4}$, with some fine-tuned FI parameters and theta angles. Namely, decomposing $\Omega=\omega_{K_{n}}+\cdots+\omega_{K_{1}}$ with $K_{n} \geq \cdots \geq K_{1}$, we find

$$
Z_{S^{2} \subset S_{b}^{4}}^{\prod_{k} \mathrm{U}\left(N_{k}\right), W_{\eta}}(m, z, \bar{z})=A a(x) a(\bar{x})\left\langle\widehat{V}_{\alpha_{\infty}}(\infty) \widehat{V}_{\hat{m}}(1) \widehat{V}_{-b \Omega}(x, \bar{x}) \widehat{V}_{\alpha_{0}}(0)\right\rangle,
$$

where $^{32} N_{k}=\sum_{j=1}^{k} K_{j}$ for $1 \leq k \leq n$,

$$
\begin{aligned}
& \eta_{n}=-1 \quad \text { and } \quad \hat{z}_{n}=x \\
& \eta_{k}=+1 \quad \text { and } \quad \hat{z}_{k}=1 \quad \text { for } 1 \leq k \leq n-1
\end{aligned}
$$

and the momenta $\alpha_{0}, \alpha_{\infty}$, and $\hat{m}$ are given by (2.119). The factor

$$
a(x) a(\bar{x})=|x|^{2 \beta}|1-x|^{2 \gamma}
$$

differs from (2.123) and has the exponents

$$
\begin{aligned}
& \beta=\langle Q,-b \Omega\rangle-\frac{N_{n}}{N_{f}} \sum_{t=1}^{N_{f}} \mathrm{i} m_{t}-\sum_{j=1}^{n-1} N_{j} b^{2}, \\
& \gamma=-b^{2} \frac{N_{n}\left(N_{f}-N_{n}\right)}{N_{f}}+\frac{N_{n}}{N_{f}} \sum_{t}\left(\mathrm{i} m_{t}+\mathrm{i} \widetilde{m}_{t}\right) .
\end{aligned}
$$

Finally, the overall constant $A$ is identical to the constant in (2.114), given by (2.122), because the three-point functions $\widehat{C}_{-b \omega_{K},-b \Omega}^{-b\left(\Omega+\omega_{K}\right)}$ are in fact all equal to 1 . Incidentally, in the case $\Omega=N h_{1}$, the factor $A a(x) a(\bar{x})$ coincides with the factor we found in the matching between the same Toda CFT correlator and the SQCDA surface operator. Thus, SQCDA and the $\mathrm{U}(N) \times \cdots \times \mathrm{U}(1)$ theory which appears in this matching have equal $S^{2}$ partition functions. The relation between these theories may run deeper.

\footnotetext{
${ }^{32}$ As explained below (2.10), the factor $A a(x) a(\bar{x})$ can be absorbed into the partition function.
} 
Since the partition function in (2.146) is known explicitly, the matching gives an explicit expression for the Toda CFT four-point function of two full, one simple, and a degenerate operator $\widehat{V}_{-b \Omega}$. The Higgs branch expansion of $Z$ provides conformal blocks as explicit series. From the Coulomb branch representation of $Z$ for $\hat{m}=0$ one can extract integral expressions for the three-point function of a degenerate operator $\widehat{V}_{-b \Omega}$ with generic vertex operators. These expressions typically involve fewer integrals than expressions obtained form the Coulomb gas formalism, but we have not investigated this direction further.

More generally, any Toda CFT $(p+3)$-point function with two generic and one semidegenerate operators at $0, \infty$ and 1 , and $p$ arbitrary degenerate operators $\widehat{V}_{-b \Omega_{l}}\left(x_{l}, \bar{x}_{l}\right)$ is equal to the partition function of a surface operator describing a certain quiver gauge theory. This matching directly derives from the matching (2.114), with only antisymmetric degenerate operators, and taking all but $p$ of the $\hat{z}$ equal to 1 . Concretely, we express each highest weight as

$$
\Omega_{l}=\sum_{j=1}^{c_{l}} \omega_{K_{l, j}}
$$

where $c_{l}$ is the number of columns in the Young diagram of $\Omega_{l}$ and $K_{l, c_{l}} \geq \cdots \geq K_{l, 2} \geq$ $K_{l, 1} \geq 0$ are the number of boxes in each column. We then define an order on the pairs $\left\{(l, j) \mid 1 \leq l \leq p, 1 \leq j \leq c_{l}\right\}$ by $(k, i) \leq(l, j)$ if $k<l$ or if $k=l$ and $i \leq j$. The gauge group is then

$$
\prod_{l=1}^{p} \prod_{j=1}^{c_{l}} \mathrm{U}\left(N_{l, j}\right) \quad \text { where } \quad N_{l, j}=\sum_{(k, i) \leq(l, j)} K_{k, i} .
$$

The matter content of the theory consists as usual of pairs of bifundamental chiral multiplets between neighboring nodes, namely $(k, i) \leftrightarrow(k, i+1)$ and $\left(k, c_{k}\right) \leftrightarrow(k+1,1)$, of an adjoint chiral multiplet for every node except $\mathrm{U}\left(N_{p, c_{p}}\right)$, and of $N_{f}$ fundamental and $N_{f}$ antifundamental chiral multiplets for this last node $\mathrm{U}\left(N_{p, c_{p}}\right)$. Complexified FI parameters associated to each node $\mathrm{U}\left(N_{l, j}\right)$ are given by

$$
\hat{z}_{l, j}= \begin{cases}1 & \text { if } 1 \leq j<c_{l}, \\ x_{l} / x_{l+1} & \text { if } j=c_{l},\end{cases}
$$

where $x_{p+1}=1$. Detailed factors can be read from the matching (2.114) using this gauge theory data.

All in all, we have identified the $\mathcal{N}=(2,2)$ surface operator corresponding to the insertion of an arbitrary set of degenerate vertex operators in a Toda CFT three-point function. It would be interesting to calculate the expectation values of such surface operators in an interacting four dimensional theory of class $\mathrm{S}$.

\section{Gauge theory dualities as Toda symmetries}

In this section, we probe low-energy dualities between two dimensional $\mathcal{N}=(2,2)$ gauge theories through the correspondence of surface operators with Toda CFT degenerate operators. In section 3.1, we show that some pairs of $\mathcal{N}=(2,2)$ SQCD theories have equal 
partition functions on $S^{2}$, as predicted by the Seiberg duality. The equality is realized as the symmetry of Toda CFT correlators under conjugation of momenta. In section 3.2, we consider $\mathcal{N}=(2,2)$ SQCDA theories with superpotentials. We focus first on a generalization of $\mathcal{N}=(2,2)^{*}$ SQCD, and find the analogue of Seiberg duality for such theories, which amounts to crossing symmetry of a Toda CFT correlator. We then obtain the Kutasov-Schwimmer duality between $\mathcal{N}=(2,2)$ SQCDA theories with a $W=\operatorname{Tr} X^{l+1}$ superpotential as conjugation of momenta. In section 3.3 finally, we describe the groupoid of Seiberg dualities for some quiver gauge theories: some dualities correspond to permutations of degenerate punctures on the Toda CFT side, and in one case to momentum conjugation.

We check all dualities by proving that the $S^{2}$ partition functions of dual theories are equal up to simple ambiguous factors: besides the Toda CFT approach, we provide direct proofs in appendix B. In all cases, the factors can be absorbed in either one of the dual partition functions through the ambiguities described below (2.10), namely a renormalization scheme ambiguity, a global gauge transformation, and a flavour FI parameter.

\subsection{Seiberg duality as momentum conjugation}

Seiberg duality relates theories with different gauge groups but the same flavour symmetry. In our two dimensional $\mathcal{N}=(2,2)$ context, it is expected that $\mathrm{U}(N)$ SQCD with $N_{f}$ fundamental and $\widetilde{N_{f}} \leq N_{f}$ antifundamental chiral multiplets is dual to $\mathrm{U}\left(N_{f}-N\right)$ SQCD with the same number of chiral multiplets and $N_{f} \widetilde{N_{f}}$ additional free mesons, for an appropriate choice of twisted masses. In the case $\widetilde{N}_{f} \leq N_{f}-2$, the series giving vortex partition functions were proven term by term to be equal in [17], and the relation for $S^{2}$ partition functions was deduced. For $\widetilde{N_{f}}=N_{f}-1$ or $\widetilde{N_{f}}=N_{f}$, vortex partition functions differ by a non-trivial factor, found numerically in [55, appendix F]. Our direct proof in appendix B.1 (similar to that of [48] found independently) is technical and by itself provides no insight on the factor. In contrast, the factor appears naturally in the proof we give here via the Toda CFT.

We denote by $m_{s}$ and $\widetilde{m}_{s}$ the twisted masses (with $R$-charges) in the electric theory, and by $m_{s}^{D}$ and $\widetilde{m}_{s}^{D}$ those in the dual magnetic theory. We shall prove that

$$
Z_{S^{2}}^{\mathrm{U}(N), N_{f}, \widetilde{N_{f}}}(z, \bar{z}, m, \widetilde{m})=a(z, \bar{z}) \prod_{s=1}^{N_{f}} \prod_{t=1}^{\widetilde{N_{f}}}\left[\gamma\left(-\mathrm{i} m_{s}-\mathrm{i} \widetilde{m}_{t}\right)\right] Z_{S^{2}}^{\mathrm{U}\left(N^{D}\right), N_{f}, \widetilde{N}_{f}}\left(z^{D}, \bar{z}^{D}, m^{D}, \widetilde{m}^{D}\right)
$$

where $z$ and $z^{D}$ are renormalized values at the scale $\ell$ of the sphere, and dual parameters are $N^{D}=N_{f}-N, z^{D}=(-1)^{\widetilde{N}_{f}} z, \bar{z}^{D}=(-1)^{\widetilde{N_{f}}} \bar{z}, m_{s}^{D}=\frac{\mathrm{i}}{2}-m_{s}$, and $\widetilde{m}_{s}^{D}=\frac{\mathrm{i}}{2}-\widetilde{m}_{s}$. The factor

$$
a(z, \bar{z})= \begin{cases}|z|^{2 \delta_{0}} & \text { if } \widetilde{N_{f}} \leq N_{f}-2, \\ |z|^{2 \delta_{0}} e^{(-1)^{N_{f}+N-1}(z-\bar{z})} & \text { if } \widetilde{N_{f}}=N_{f}-1 \\ |z|^{2 \delta_{0}}\left|1-(-1)^{N_{f}+N-1} z\right|^{2 \delta_{1}} & \text { if } \widetilde{N_{f}}=N_{f}\end{cases}
$$


is given in terms of the exponents

$$
\begin{aligned}
& \delta_{0}=\gamma_{0}-\gamma_{0}^{D}=-\frac{N_{f}-N}{2}-\sum_{s=1}^{N_{f}} \mathrm{i} m_{s}, \\
& \delta_{1}=\gamma_{1}-\gamma_{1}^{D}=N_{f}-N+\sum_{s=1}^{N_{f}}\left(\mathrm{i} m_{s}+\mathrm{i} \widetilde{m}_{s}\right),
\end{aligned}
$$

which we will obtain from the exponents $\gamma_{i}$ in the matching (2.51), and their duals $\gamma_{i}^{D}$. The factor $a(z, \bar{z})$ could be absorbed into $Z$ in three parts as discussed below (2.10). First, a renormalization scheme ambiguity absorbs any factor independent of twisted masses. Next, a global gauge transformation shifts the partition function by any power of $|z|^{2}$. A last factor (present only for $\widetilde{N_{f}}=N_{f}$ ) can be absorbed by turning on an FI parameter for the $\mathrm{U}(1)$ flavour group under which fundamental and antifundamental chiral multiplets have the same charge.

The product of gamma functions in (3.1) is the (one-loop determinant) contribution from $N_{f} \widetilde{N_{f}}$ free mesons with twisted masses $m_{s}+\widetilde{m}_{t}=\mathrm{i}-m_{s}^{D}-\widetilde{m}_{t}^{D}$. These twisted masses are equal to those of the mesons $\widetilde{q}_{t} q_{s}$, where $q_{s}$ and $\widetilde{q}_{t}$ are fundamental and antifundamental quarks of the electric theory. In the magnetic theory, the twisted masses derive from the superpotential coupling $W=\widetilde{q}^{D} M q^{D}$, which has total $R$-charge 2, hence total (complexified) twisted mass $\widetilde{m}_{t}^{D}+\left(\mathrm{i}-m_{s}^{D}-\widetilde{m}_{t}^{D}\right)+m_{s}^{D}=\mathrm{i}$.

Applied twice, the duality (3.1) yields the original theory, since parameters are mapped back to those of the electric theory. An immediate consistency check is thus

$$
\gamma\left(-\mathrm{i} m_{s}-\mathrm{i} \widetilde{m}_{t}\right) \gamma\left(-\mathrm{i} m_{s}^{D}-\mathrm{i} \widetilde{m}_{t}^{D}\right)=\frac{\Gamma\left(-\mathrm{i} m_{s}-\mathrm{i} \widetilde{m}_{t}\right)}{\Gamma\left(1+\mathrm{i} m_{s}+\mathrm{i} \widetilde{m}_{t}\right)} \frac{\Gamma\left(1+\mathrm{i} m_{s}+\mathrm{i} \widetilde{m}_{t}\right)}{\Gamma\left(-\mathrm{i} m_{s}-\mathrm{i} \widetilde{m}_{t}\right)}=1
$$

and that the $a(z, \bar{z})$ factors cancel thanks to

$$
\begin{aligned}
& \delta_{0}^{D}=-\frac{N_{f}-N^{D}}{2}-\sum_{s=1}^{N_{f}} \mathrm{i} m_{s}^{D}=-\frac{N}{2}+\sum_{s=1}^{N_{f}} \mathrm{i} m_{s}+\frac{N_{f}}{2}=-\delta_{0}, \\
& \delta_{1}^{D}=N_{f}-N^{D}+\sum_{s=1}^{N_{f}}\left(\mathrm{i} m_{s}^{D}+\mathrm{i} \widetilde{m}_{s}^{D}\right)=N-\sum_{s=1}^{N_{f}}\left(\mathrm{i} m_{s}+\mathrm{i} \widetilde{m}_{s}\right)-N_{f}=-\delta_{1},
\end{aligned}
$$

and, for $\widetilde{N_{f}}=N_{f}-1,(-1)^{N_{f}+N^{D}-1}\left(z^{D}-\bar{z}^{D}\right)=-(-1)^{N_{f}+N-1}(z-\bar{z})$. A second consistency check, in the case $\widetilde{N_{f}}=N_{f}$, is the symmetry under charge conjugation $z \leftrightarrow 1 / z, \bar{z} \leftrightarrow 1 / \bar{z}$, and $\mathrm{i} m_{s} \leftrightarrow \mathrm{i} \widetilde{m}_{s}$. We find that $\delta_{1}$ is left unchanged, and that $\delta_{0}$ is mapped to $-\delta_{0}-\delta_{1}$, consistent with $a(1 / z, 1 / \bar{z})=|z|^{-2 \delta_{0}-2 \delta_{1}}\left|1-(-1)^{N_{f}+N-1} z\right|^{2 \delta_{1}}$.

\subsubsection{Momentum conjugation for $\widetilde{N}_{f}=N_{f}$}

To derive the Seiberg duality relation (3.1) for $\widetilde{N_{f}}=N_{f}$, we rely on the matching (2.51) relating the $S^{2}$ partition function of $\mathrm{U}(N)$ SQCD to a Toda CFT four-point function:

$$
Z_{S^{2} \subset S_{b}^{4}}^{\mathrm{U}(N) \widetilde{N}_{f}=N_{f}}(m, \widetilde{m}, z, \bar{z})=A|x|^{2 \gamma_{0}}|1-x|^{2 \gamma_{1}}\left\langle\widehat{V}_{\alpha_{\infty}}(\infty) \widehat{V}_{\hat{m}}(1) \widehat{V}_{-b \omega_{N}}(x, \bar{x}) \widehat{V}_{\alpha_{0}}(0)\right\rangle .
$$


The four-point function features two generic operators $\widehat{V}_{\alpha}$, one semi-degenerate operator $\widehat{V}_{\hat{m}}$, and the degenerate operator $\widehat{V}_{-b \omega_{N}}$ inserted at $x=(-1)^{N_{f}+N-1} z$ and labeled by the highest weight $\omega_{N}$ of the $N$-th antisymmetric representation of $A_{N_{f}-1}$. The relation between gauge theory twisted masses $m$ and $\widetilde{m}$, and Toda CFT momenta $\alpha_{0}, \alpha_{\infty}$, and $\hat{m}$ is given in section 2.2 .

Toda CFT correlators are invariant under changing all momenta to their conjugate, that is, applying the $\mathbb{C}$-linear transformation $h_{s} \rightarrow h_{s}^{C}=-h_{N_{f}+1-s}$ which maps weights of a representation of $A_{N_{f}-1}$ to weights of the conjugate representation. This transformation maps the degenerate momentum $-b \omega_{N}$ to ${ }^{33}$

$$
\left(-b \omega_{N}\right)^{C}=-\sum_{s=1}^{N} b h_{s}^{C}=\sum_{s=N_{f}-N+1}^{N_{f}} b h_{s}=-\sum_{s=1}^{N_{f}-N} b h_{s}=-b \omega_{N_{f}-N},
$$

which is precisely the degenerate momentum appearing in the description of the Seiberg dual SQCD theory. The semi-degenerate momentum $\hat{m}=(\varkappa+N b) h_{1}$ becomes $\hat{m}^{C}=$ $-(\varkappa+N b) h_{N_{f}}$, which is not along $h_{1}$. However, the Weyl reflection defined by the cyclic permutation of $\llbracket 1, N_{f} \rrbracket$ maps $\hat{m}^{C}$ to

$$
\left[N_{f}\left(b+\frac{1}{b}\right)-\varkappa-N b\right] h_{1}=\left(\varkappa^{D}+N^{D} b\right) h_{1}=\hat{m}^{D}
$$

where $\varkappa^{D}=N_{f} / b-\varkappa$. Indeed, $\left\langle\hat{m}^{C}-Q, h_{s}\right\rangle=\left\langle\hat{m}^{D}-Q, h_{s+1}\right\rangle$ for all $1 \leq s \leq N_{f}-1$, and $\left\langle\hat{m}^{C}-Q, h_{N_{f}}\right\rangle=\left\langle\hat{m}^{D}-Q, h_{1}\right\rangle$. Finally, the generic momenta $\alpha_{0}$ and $\alpha_{\infty}$ remain generic after conjugation, and we have

$$
\left\langle\alpha^{C}-Q, h_{p}\right\rangle=\left\langle\alpha-Q^{C}, h_{p}^{C}\right\rangle=-\left\langle\alpha-Q, h_{N_{f}+1-p}\right\rangle,
$$

where we used that $\left\langle\alpha_{1}, \alpha_{2}\right\rangle=\left\langle\alpha_{1}^{C}, \alpha_{2}^{C}\right\rangle$ and that $Q=Q^{C}$. A Weyl reflection then permutes $\left\langle\alpha-Q, h_{N_{f}+1-p}\right\rangle \rightarrow\left\langle\alpha-Q, h_{p}\right\rangle$, hence conjugation followed by this reflection simply changes $\alpha \rightarrow 2 Q-\alpha$.

We thus find that conjugation of all momenta (with subsequent Weyl reflections) relates two correlators which correspond to SQCD with $\mathrm{U}(N)$ and $\mathrm{U}\left(N_{f}-N\right)$ gauge groups. Converting the relation between momenta to gauge theory variables, we find $m_{s}^{D}=\frac{\mathrm{i}}{2}-m_{s}$ and $\widetilde{m}_{s}^{D}=\frac{\mathrm{i}}{2}-\widetilde{m}_{s}$, as we claimed below $(3.1) .{ }^{34}$

In our normalization, both generic and non-degenerate operators are Weyl reflection invariant, without reflection amplitudes. The two Toda CFT correlators are thus equal, and we divide the factor relating the $S^{2}$ partition functions and Toda CFT correlator for one theory by the factor for the other theory to find (for $\widetilde{N_{f}}=N_{f}$ )

$$
Z_{S^{2}}^{\mathrm{U}(N)}(z, \bar{z}, m, \widetilde{m})=\frac{Z_{S_{b}^{4}}^{\mathrm{free}, D} A|x|^{2 \gamma_{0}}|1-x|^{2 \gamma_{1}}}{Z_{S_{b}^{4}}^{\text {free }} A^{D}|x|^{2 \gamma_{0}^{D}}|1-x|^{2 \gamma_{1}^{D}}} Z_{S^{2}}^{\mathrm{U}\left(N^{D}\right)}\left(z^{D}, \bar{z}^{D}, m^{D}, \widetilde{m}^{D}\right) .
$$

\footnotetext{
${ }^{33}$ The third step uses that the weights $h_{s}$ of the fundamental representation of $A_{N_{f}-1}$ sum to zero.

${ }^{34} \mathrm{~A}$ global U(1) gauge transformation is identical to the flavour symmetry which shifts $\mathrm{i} m_{s}$ and $-\mathrm{i} \widetilde{m}_{s}$ by the same amount. This has no physical effect: the Toda correlator is invariant, and the partition function is multiplied by a power of $|z|^{2}$. Dual twisted masses are only defined up to such a shift, which also alters $\delta_{0}$.
} 
We recognize the factor $a(z, \bar{z})=|z|^{2 \gamma_{0}-2 \gamma_{0}^{D}}\left|1-(-1)^{N_{f}+N-1} z\right|^{2 \gamma_{1}-2 \gamma_{1}^{D}}$ announced in (3.2). The ratio $A / A^{D}$ simplifies:

$$
\frac{A}{A^{D}}=\frac{b^{N N_{f}\left(1+b^{2}\right)-N^{2} b^{2}-2 N \sum_{s=1}^{N_{f}}\left(1+\mathrm{i} m_{s}+\mathrm{i} \widetilde{m}_{s}\right)}}{b^{N^{D} N_{f}\left(1+b^{2}\right)-\left(N^{D}\right)^{2} b^{2}-2 N^{D} \sum_{s=1}^{N_{f}}\left(1+\mathrm{i} m_{s}^{D}+\mathrm{i} \widetilde{m}_{s}^{D}\right)}}=b^{-N_{f} \sum_{s=1}^{N_{f}}\left(1+2 \mathrm{i} m_{s}+2 \mathrm{i} \widetilde{m}_{s}\right)} .
$$

The hypermultiplets masses (2.17) involved in the $S_{b}^{4}$ partition functions (2.4) associated to the electric and magnetic theories are

$$
\begin{aligned}
& m_{s t}=\mathrm{i} \frac{1-b^{2}}{2 b}-\frac{1}{b}\left(m_{s}+\widetilde{m}_{t}\right), \\
& m_{s t}^{D}=\mathrm{i} \frac{1-b^{2}}{2 b}-\frac{1}{b}\left(\mathrm{i}-m_{s}-\widetilde{m}_{t}\right)=-\mathrm{i} b-m_{s t},
\end{aligned}
$$

thus the constant factor is

$$
\begin{aligned}
\frac{Z_{S_{b}^{4}}^{\mathrm{free}, D} A}{Z_{S_{b}^{4}}^{\text {free }} A^{D}} & =\frac{A}{A^{D}} \prod_{s, t} \frac{\Upsilon\left(\frac{b}{2}+\frac{1}{2 b}-\mathrm{i} m_{s t}\right)}{\Upsilon\left(\frac{b}{2}+\frac{1}{2 b}-\mathrm{i} m_{s t}^{D}\right)}=\frac{A}{A^{D}} \prod_{s, t} \frac{\Upsilon\left(\frac{b}{2}+\frac{1}{2 b}+\mathrm{i} m_{s t}\right)}{\Upsilon\left(\frac{b}{2}+\frac{1}{2 b}+\mathrm{i} m_{s t}-b\right)} \\
& =b^{-N_{f} \sum_{s=1}^{N_{f}}\left(1+2 \mathrm{i} m_{s}+2 \mathrm{i} \widetilde{m}_{s}\right)} \prod_{s, t}\left[b^{b^{2}-2 b \mathrm{i} m_{s t}} \gamma\left(\frac{1-b^{2}}{2}+b \mathrm{i} m_{s t}\right)\right] \\
& =\prod_{s, t} \gamma\left(-\mathrm{i} m_{s}-\mathrm{i} \widetilde{m}_{t}\right) .
\end{aligned}
$$

The one-loop determinants of free mesons appear here thanks to the shift by $b$ in i $m_{s t}^{D}=$ $b-\mathrm{i} m_{s t}$, which relies on the shift between $m_{s t}$ and $\frac{-1}{b}\left(m_{s}+\widetilde{m}_{t}\right)$ in (2.17). We obtain this constant factor more directly for any $\widetilde{N_{f}} \leq N_{f}$ in the next section.

\subsubsection{Decoupling multiplets towards $\widetilde{N_{f}}<N_{f}$}

We could find analogous Seiberg duality relations for $\widetilde{N_{f}}<N_{f}$ via the matching of section 2.2.3 with Toda irregular punctures, but those cases are also easily accessed by taking some twisted masses of antifundamental multiplets to be very large in the $\widetilde{N_{f}}=N_{f}$ duality. The reverse process, which decreases $N_{f}>\widetilde{N_{f}}$ by giving some fundamental multiplets large twisted masses, is more difficult, and must be significantly altered to reach the case $N_{f}=\widetilde{N_{f}}$ in appendix B.1.

Our starting point to prove (3.1) is the Higgs branch decomposition of the $S^{2}$ partition function of interest $[17,18]$ :

$$
\begin{aligned}
Z^{\mathrm{U}(N), N_{f}, \widetilde{N}_{f}} & =\sum_{p_{1}<\cdots<p_{N}}^{N_{f}}(z \bar{z})^{-\sum_{j=1}^{N} \mathrm{i} m_{p_{j}}} Z_{11,\{p\}}^{N_{f}, \widetilde{N}_{f}} f_{\{p\}}^{(\mathrm{s}), N_{f}, \widetilde{N}_{f}}\left((-1)^{N_{f}+N-1} z\right) f_{\{p\}}^{(\mathrm{s}), N_{f}, \widetilde{N}_{f}}\left((-1)^{\widetilde{N}_{f}}+N-1 \bar{z}\right), \\
Z_{11,\{p\}}^{N_{f}, \widetilde{N}_{f}}=\prod_{j=1}^{N} \frac{\prod_{s \notin\{p\}}^{N_{f}} \gamma\left(-\mathrm{i} m_{s}+\mathrm{i} m_{p_{j}}\right)}{\prod_{s=1}^{N_{f}} \gamma\left(1+\mathrm{i} \widetilde{m}_{s}+\mathrm{i} m_{p_{j}}\right)}, & f_{\{p\}}^{(\mathrm{s}), N_{f}, \widetilde{N}_{f}}(x)=\sum_{k=0}^{\infty} \frac{x^{k}}{k !} f_{\{p\}, k}^{(\mathrm{s}), N_{f}, \widetilde{N_{f}}}, \\
f_{\{p\}, k}^{(\mathrm{s}), N_{f}, \widetilde{N_{f}}}= & k ! \sum_{k_{1}+\cdots+k_{N}=k} \prod_{j=1}^{N}\left[\frac{\prod_{s=1}^{N_{f}}\left(-\mathrm{i} \widetilde{m}_{s}-\mathrm{i} m_{p_{j}}\right)_{k_{j}}}{k_{j} ! \prod_{i \neq j}^{N}\left(\mathrm{i} m_{p_{i}}-\mathrm{i} m_{p_{j}}-k_{i}\right)_{k_{j}} \prod_{s \notin\{p\}}^{N_{f}}\left(1+\mathrm{i} m_{s}-\mathrm{i} m_{p_{j}}\right)_{k_{j}}}\right],
\end{aligned}
$$


which generalizes (2.58) to $\widetilde{N_{f}}<N_{f}$. The series $f_{\{p\}}^{(\mathrm{s}), N_{f}, \widetilde{N_{f}}}(x)$ converge on the unit disc if $\widetilde{N_{f}}=N_{f}$, and on the whole complex plane if $\widetilde{N_{f}}<N_{f}$. We shall equate the term of (3.18) labeled by $\{p\} \subseteq \llbracket 1, N_{f} \rrbracket$ with the term labeled by the complement $\{p\}^{\complement}$ for the dual theory. The powers of $|z|^{2}$ match:

$$
-\sum_{j=1}^{N} \mathrm{i} m_{p_{j}}=-\sum_{s=1}^{N_{f}} \mathrm{i} m_{s}+\sum_{s \in\{p\}^{\complement}} \mathrm{i} m_{s}=-\sum_{s=1}^{N_{f}} \mathrm{i} m_{s}-\frac{N_{f}-N}{2}-\sum_{s \in\{p\}^{\complement}} \mathrm{i} m_{s}^{D}=\delta_{0}-\sum_{s \in\{p\}^{\complement}} \mathrm{i} m_{s}^{D} .
$$

The constant is fixed as the ratio of one-loop determinants $Z_{11}$

$$
\frac{Z_{11,\{p\}}^{N_{f}, \widetilde{N_{f}}}}{Z_{11,\{p\}^{\mathrm{C}}}^{N_{f}, \widetilde{N}_{f}, D}}=\prod_{s=1}^{\widetilde{N_{f}}} \frac{\prod_{t \in\{p\}^{\mathrm{C}}} \gamma\left(1+\mathrm{i} \widetilde{m}_{s}^{D}+\mathrm{i} m_{t}^{D}\right)}{\prod_{t \in\{p\}} \gamma\left(1+\mathrm{i} \widetilde{m}_{s}+\mathrm{i} m_{t}\right)}=\prod_{s=1}^{\widetilde{N_{f}}} \prod_{t=1}^{N_{f}} \gamma\left(-\mathrm{i} \widetilde{m}_{s}-\mathrm{i} m_{t}\right)
$$

which is independent of $\{p\}$. There remains to match vortex partition functions:

$$
f_{\{p\}}^{(\mathrm{s}), N_{f}, \widetilde{N}_{f}}(x)=a(x) f_{\{p\}^{\mathrm{C}}}^{(\mathrm{s}), N_{f}, \widetilde{N}_{f}, D}\left(x^{D}\right),
$$

where

$$
a(x)= \begin{cases}(1-x)^{\delta_{1}} & \text { if } \widetilde{N_{f}}=N_{f}, \\ e^{x} & \text { if } \widetilde{N_{f}}=N_{f}-1, \\ 1 & \text { if } \widetilde{N_{f}} \leq N_{f}-2,\end{cases}
$$

and $x^{D}=(-1)^{N_{f}+N^{D}-1} z^{D}=(-1)^{\widetilde{N_{f}}+N-1} z=(-1)^{N_{f}-\widetilde{N}_{f}} x$. From the case $\widetilde{N_{f}}=N_{f}$ studied in the previous section, we now derive the relations for $\widetilde{N_{f}}<N_{f}$ by taking a limit where $N_{f}-\widetilde{N_{f}}$ antifundamental chiral multiplets are given large twisted masses. We give a proof independent of the Toda CFT matching in appendix B.1.

Let us expand $f_{\{p\}, k}^{(\mathrm{s}), N_{f}, \widetilde{N_{f}}}$, for some $\widetilde{N_{f}} \leq N_{f}$, in the limit $\widetilde{m}_{\widetilde{N}_{f}}=\Lambda \rightarrow+\infty$. This relies on the asymptotic behavior $(\rho+a)_{k} \sim \rho^{k}$ of Pochhammer symbols when $|\rho| \rightarrow \infty$ :

$$
f_{\{p\}, k}^{(\mathrm{s}), N_{f}, \widetilde{N_{f}}} \sim(-\mathrm{i} \Lambda)^{k} f_{\{p\}, k}^{(\mathrm{s}), N_{f}, \widetilde{N_{f}}-1} .
$$

Summing over $k \geq 0$,

$$
f_{\{p\}}^{(\mathrm{s}), N_{f}, \widetilde{N_{f}}}\left(\frac{x}{-\mathrm{i} \Lambda}\right) \rightarrow f_{\{p\}}^{(\mathrm{s}), N_{f}, \widetilde{N_{f}}-1}(x),
$$

as $\Lambda \rightarrow \infty$, and for a fixed $x$. We then apply this limit to (3.21) for $\widetilde{N}_{f}=N_{f}$ after changing $x \rightarrow x /(-\mathrm{i} \Lambda)$. Since $\delta_{1} \sim \mathrm{i} \Lambda$, we get $a\left(\widetilde{N}_{f}=N_{f}, \mathrm{i} x / \Lambda\right)=e^{\delta_{1} \ln (1-\mathrm{i} x / \Lambda)} \sim e^{x}$, which is the exponential factor for $\widetilde{N_{f}}=N_{f}-1$. In the limit where further twisted masses become very large while keeping the appropriate combination $-\mathrm{i} \Lambda x$ fixed, the exponential factor becomes $e^{x /(-\mathrm{i} \Lambda)} \rightarrow 1$, yielding $a(x)=1$ for $\widetilde{N}_{f} \leq N_{f}-2$. The relative sign between $x$ and $x^{D}$ is due to the sign difference $\mathrm{i} \widetilde{m}^{D} \sim-\mathrm{i} \widetilde{m}$ for each of the $N_{f}-\widetilde{N}_{f}$ antifundamental multiplets which we decouple.

This concludes the proof of the Seiberg duality relation (3.1) for all $\widetilde{N_{f}} \leq N_{f}$ as limits of the case $\widetilde{N_{f}}=N_{f}$, itself derived from the correspondence with the Toda CFT. 


\subsection{SQCDA dualities: crossing symmetry and conjugation}

In this section, we find two new Seiberg-like dualities between pairs of $\mathcal{N}=(2,2)$ theories with adjoint matter and a superpotential. The first is realized in the Toda CFT as crossing symmetry, and contains as a special case a duality between $\mathcal{N}=(2,2)^{*}$ theories, proposed in [59] for particular twisted masses, and recently in [48]. The second is realized as conjugation symmetry, and is a direct two dimensional analogue of the four dimensional Kutasov-Schwimmer duality $[52,53]$. We test both dualities by comparing $S^{2}$ partition functions using the matching with Toda CFT correlators. We also provide direct proofs that the $S^{2}$ partition functions of dual theories are equal, without relying on the Toda CFT. Namely, classical and one-loop contributions are compared in the main text, and vortex partition functions in appendix B.2. It would be interesting to work out the mapping of chiral rings of dual theories.

Each duality relates theories with $\mathrm{U}(N)$ and $\mathrm{U}\left(N^{D}\right)$ vector multiplets coupled to one adjoint, $N_{f}$ fundamental, and $\widetilde{N_{f}}$ antifundamental chiral multiplets. We assume by symmetry that $\widetilde{N}_{f} \leq N_{f}$. As for the Seiberg duality, the magnetic theory includes additional free matter. In the electric theory, chiral multiplets are denoted by $X, q_{t}$, and $\widetilde{q}_{t}$, and their (complexified) twisted masses by $m_{X}, m_{t}$, and $\widetilde{m}_{t}$ respectively. The FI parameters and theta angles (renormalized, at the scale $\ell$ of the sphere) are combined as usual into a complex parameter $z$. We use the notations $X^{D}, q_{t}^{D}, \widetilde{q}_{t}^{D}, m_{X}^{D}, m_{t}^{D}, \widetilde{m}_{t}^{D}$, and $z^{D}$ for the magnetic theory.

Recall that when $\widetilde{N_{f}}=N_{f}$ we have the matching

$$
Z_{S^{2} \subset S_{b}^{4}}^{\mathrm{U}(N C D A}\left(m, \widetilde{m}, m_{X}, z, \bar{z}\right)=A|y|^{2 \gamma_{0}}|1-y|^{2 \gamma_{1}}\left\langle\widehat{V}_{\alpha_{\infty}}(\infty) \widehat{V}_{\hat{m}}(1) \widehat{V}_{-N b h_{1}}(y, \bar{y}) \widehat{V}_{\alpha_{0}}(0)\right\rangle
$$

for $y=(-1)^{N_{f}} z, b^{2}=\mathrm{i} m_{X}$, with other parameters given below (2.88). The four-point function can exhibit two types of symmetries. If the semi-degenerate momentum $\hat{m}=$ $(\varkappa+N b) h_{1}$ is in fact degenerate $\left(\hat{m}=-N^{D} b h_{1}\right)$, then crossing symmetry exchanges the two degenerate operators via $N \leftrightarrow N^{D}$ and $y \rightarrow y^{-1}$. This yields the duality in section 3.2.1. On the other hand, it turns out that for fined-tuned values $\mathrm{i} m_{X}=b^{2}=\frac{-1}{l+1}$ the degenerate operator $\widehat{V}_{-N b h_{1}}$ is conjugate to another degenerate operator, $\widehat{V}_{-N^{D} b h_{1}}$. This leads to the Kutasov-Schwimmer duality in section 3.2.2, which we then extend to $\widetilde{N}_{f}<N_{f}$ as we did for the Seiberg duality.

\subsection{1 $(2,2)^{*}$-like duality as a braiding move}

Let us describe the first duality more precisely. With notations as above, the electric and magnetic theories are $\mathcal{N}=(2,2)$ SQCDA theories with $N$ and $N^{D}$ colors and the same matter content and superpotential

$$
W=\sum_{t=1}^{N_{f}} \widetilde{q}_{t} X^{l_{t}} q_{t} \quad \text { hence } \quad 1+\mathrm{i} m_{t}+\mathrm{i} \widetilde{m}_{t}+l_{t} \mathrm{i} m_{X}=0
$$

where $l_{t} \geq 0$ are integers, and $\widetilde{N_{f}}=N_{f}$. We will find that $N^{D}=L-N$ with $L=\sum_{t=1}^{N_{f}} l_{t}$, twisted masses are the same in the two theories, $z^{D}=z^{-1}$, and $\bar{z}^{D}=\bar{z}^{-1}$. 
In particular, when all $l_{t}=1$ the theories are $\mathcal{N}=(2,2)^{*}$ SQCD with gauge groups $\mathrm{U}(N)$ and $\mathrm{U}\left(N_{f}-N\right)$, and the duality is an $\mathcal{N}=(2,2)^{*}$ analogue of the $\mathcal{N}=(2,2)$ Seiberg duality found earlier. In the special case $m_{X}=\mathrm{i} / 2$, the two dualities agree after charge conjugation, which exchanges $m_{s}^{D} \leftrightarrow \widetilde{m}_{s}^{D}$ and maps $z^{D} \rightarrow\left(z^{D}\right)^{-1}$. The agreement is expected since the superpotential term $W=\operatorname{Tr} X^{2}$ is then supersymmetric and $X$ can be integrated out, shifting the theta angle by $(N-1) \pi$ in the process: this leads to a sign difference in the maps $z \rightarrow z^{D}$ of the two dualities.

We test the duality by proving that $S^{2}$ partition functions match:

$$
Z_{S^{2}}^{\mathrm{U}(N)}(z, \bar{z})=|z|^{2 \delta_{0}}\left|1-(-1)^{N_{f}} z\right|^{2 \delta_{1}} Z_{S^{2}}^{\mathrm{U}\left(N^{D}\right)}\left(z^{D}, \bar{z}^{D}\right) \quad \text { for } W=\sum_{t=1}^{N_{f}} \widetilde{q}_{t} X^{l_{t}} q_{t}
$$

with dual parameters given above, and the exponents

$$
\begin{aligned}
& \delta_{0}=-(L-N)\left(1+\mathrm{i} m_{X}\right)-\sum_{t=1}^{N_{f}} \sum_{\nu=0}^{l_{t}-1}\left(\mathrm{i} m_{t}+\nu \mathrm{i} m_{X}\right), \\
& \delta_{1}=(L-2 N)\left(1+\mathrm{i} m_{X}\right) .
\end{aligned}
$$

As discussed below (3.2) for the Seiberg duality, the powers of $|z|^{2}$ and $\left|1-(-1)^{N_{f}} z\right|^{2}$ can be absorbed as ambiguities of the $S^{2}$ partition function. ${ }^{35}$ The same consistency checks as for the Seiberg duality apply. Repeating the duality yields the original parameters, and the factors cancel thanks to $\delta_{0}^{D}=\delta_{0}+\delta_{1}$ and $\delta_{1}^{D}=-\delta_{1}$. The relation is also invariant under charge conjugation, which exchanges twisted masses of fundamental and antifundamental chiral multiplets, since $\delta_{1}$ is unchanged and $\delta_{0} \rightarrow-\delta_{0}-\delta_{1}$. We first derive dual parameters from the matching of SQCDA partition functions to Toda CFT correlators. For completeness, we then prove the relation by comparing classical, one-loop and vortex contributions of the two theories.

Recall the matching (2.88) between the partition function of a sphere surface operator describing $\mathrm{U}(N)$ SQCDA and a Toda CFT correlator with the symmetric degenerate operator $\widehat{V}_{-N b h_{1}}$, a semi-degenerate operator $\widehat{V}_{\hat{m}}$, and two generic operators. We find in section 2.3.3 that the superpotential $W=\sum_{t} \widetilde{q}_{t} X^{l_{t}} q_{t}$ constrains twisted masses in such a way that $\hat{m}=-(L-N) b h_{1}=-N^{D} b h_{1}$. The $S^{2}$ partition function of the electric theory we are studying is thus

$$
Z_{S^{2}}^{\mathrm{U}(N)}(z, \bar{z})=\widetilde{A}|y|^{2 \gamma_{0}}|1-y|^{2 \gamma_{1}}\left\langle\widehat{V}_{\alpha_{\infty}}(\infty) \widehat{V}_{-N^{D} b h_{1}}(1) \widehat{V}_{-N b h_{1}}(y, \bar{y}) \widehat{V}_{\alpha_{0}}(0)\right\rangle
$$

where $y=(-1)^{N_{f}} z, b^{2}=\mathrm{i} m_{X}$, momenta and exponents are given below (2.88), and we have absorbed in $\widetilde{A}$ the contributions from the $S_{b}^{4}$ hypermultiplets and from the differing normalization of semidegenerate and degenerate operators. The Toda CFT correlator is invariant under $N \rightarrow N^{D}, y \rightarrow y^{D}=y^{-1}$, and the conformal map $\left(\infty, 1, y^{-1}, 0\right) \rightarrow$

\footnotetext{
${ }^{35}$ For $\mathcal{N}=(2,2)^{*}$ theories, the power of $1-(-1)^{N_{f}} z$ relating dual vortex partition functions was found numerically by Honda and Okuda [55].
} 
$(\infty, y, 1,0)$. This implies that

$$
Z_{S^{2}}^{\mathrm{U}(N)}(z, \bar{z})=\frac{\widetilde{A}|y|^{2 \gamma_{0}}|1-y|^{2 \gamma_{1}}}{\widetilde{A}^{D}\left|y^{D}\right|^{2 \gamma_{0}^{D}}\left|1-y^{D}\right|^{2 \gamma_{1}^{D}}}|y|^{\Delta\left(\alpha_{\infty}\right)-\Delta\left(-N^{D} b h_{1}\right)-\Delta\left(-N b h_{1}\right)-\Delta\left(\alpha_{0}\right)} Z_{S^{2}}^{\mathrm{U}\left(N^{D}\right)}\left(z^{D}, \bar{z}^{D}\right) \text {. }
$$

We deduce the exponents (3.28) and (3.29) by computing $\delta_{1}=\gamma_{1}-\gamma_{1}^{D}$ and

$$
\delta_{0}=\gamma_{0}+\Delta\left(\alpha_{\infty}\right)-\Delta\left(-N^{D} b h_{1}\right)-\Delta\left(-N b h_{1}\right)-\Delta\left(\alpha_{0}\right)+\gamma_{0}^{D}+\gamma_{1}^{D} .
$$

We also obtain $z^{D}=(-1)^{N_{f}} y^{D}=(-1)^{N_{f}} y^{-1}=z^{-1}$ and $N^{D}=L-N$ as announced.

There remains to fix the overall constant factor, since $\widetilde{A} / \widetilde{A}^{D}$ is difficult to evaluate ( $\widetilde{A}$ and $\widetilde{A}^{D}$ are singular for our choice of twisted masses). This is done by comparing the s-channel decomposition (as $z \rightarrow 0$ ) of the electric theory with the u-channel decomposition (as $z^{D} \rightarrow \infty$ ) of the magnetic theory. Recall from section 2.3.3 that the s-channel Higgs branch vacua of the electric theory are labeled by ordered partitions $\sum_{t=1}^{N_{f}} n_{t}=N$ with $0 \leq n_{t} \leq l_{t}$. The classical and one-loop contributions (2.96) are

$$
\begin{aligned}
Z_{\mathrm{cl},\left\{n_{t}\right\}}^{(\mathrm{s})}(z, \bar{z}) & =(z \bar{z})^{\sum_{s=1}^{N_{f}} \sum_{\mu=0}^{n_{s}-1}\left(-\mathrm{i} m_{s}-\mu \mathrm{i} m_{X}\right)}, \\
Z_{11,\left\{n_{t}\right\}}^{(\mathrm{s})} & =\prod_{s=1}^{N_{f}} \prod_{\mu=0}^{n_{s}-1} \prod_{t=1}^{N_{f}} \frac{\gamma\left(\mathrm{i} m_{s}-\mathrm{i} m_{t}+\left(\mu-n_{t}\right) \mathrm{i} m_{X}\right)}{\gamma\left(\mathrm{i} m_{s}-\mathrm{i} m_{t}+\left(\mu-l_{t}\right) \mathrm{i} m_{X}\right)} .
\end{aligned}
$$

Similarly, u-channel Higgs branch vacua of the magnetic theory are labeled by partitions $\sum_{t=1}^{N_{f}} n_{t}^{D}=N^{D}$ with $0 \leq n_{t}^{D} \leq l_{t}$, and are in a natural bijection with those of the electric theory through $n_{t}^{D}=l_{t}-n_{t}$. The classical contributions match up to $|z|^{2 \delta_{0}}$ :

$$
\sum_{s=1}^{N_{f}} \sum_{\mu=0}^{n_{s}-1}\left(-\mathrm{i} m_{s}-\mu \mathrm{i} m_{X}\right)=\delta_{0}-\sum_{s=1}^{N_{f}} \sum_{\mu=0}^{l_{s}-n_{s}-1}\left(\mathrm{i} \tilde{m}_{s}+\mu \mathrm{i} m_{X}\right) .
$$

The one-loop contributions are equal, with no relative constant factor, since

$$
Z_{11,\left\{n_{t}\right\}}^{(\mathrm{s})}=\prod_{s=1}^{N_{f}} \prod_{\mu=0}^{n_{s}-1} \prod_{t=1}^{N_{f}} \prod_{\nu=0}^{l_{t}-n_{t}-1} \frac{\gamma\left(1+\mathrm{i} m_{s}+\mathrm{i} \widetilde{m}_{t}+(\mu+\nu+1) \mathrm{i} m_{X}\right)}{\gamma\left(1+\mathrm{i} m_{s}+\mathrm{i} \widetilde{m}_{t}+(\mu+\nu) \mathrm{i} m_{X}\right)}
$$

is invariant under $m \leftrightarrow \widetilde{m}$ and $n \rightarrow l-n$. We prove in appendix B.2 that the vortex partition functions match up to $(1-y)^{\delta_{1}}$. This establishes the duality relation (3.27).

From the duality we can extract information about powers of $|1-y|^{2}$ which appear in the expansion of $Z$ near $y=1$. In the electric theory, the powers are given by (2.100), valid for all SQCDA theories: replacing $k$ by $N-k$ there,

$$
Z_{S^{2}}^{\mathrm{U}(N)}(z, \bar{z})=|1-y|^{-2 N\left(1+\mathrm{i} m_{X}\right)} \sum_{k=0}^{N}\left[|1-y|^{2\left[k\left(1+\mathrm{i} m_{X}\right)-(N-k)\left(N^{D}-k\right) \mathrm{i} m_{X}\right]}(\text { series })\right]
$$

for some series in non-negative powers of $(1-y)$ and $(1-\bar{y})$. The magnetic theory has a similar expansion with $N \leftrightarrow N^{D}$. Since the two must match, we deduce that the expansion (3.37) holds, with a sum restricted to $0 \leq k \leq \min \left(N, N^{D}\right)$. This list of exponents is useful to identify the correct relation between quiver gauge theories and correlators in section 2.4 . 


\subsubsection{Kutasov-Schwimmer duality as momentum conjugation}

The Kutasov-Schwimmer duality [52, 53], initially proposed between four dimensional theories, is similar to the Seiberg duality, with an additional adjoint matter multiplet $X$ subject to the superpotential coupling $W=\operatorname{Tr} X^{l+1}$. Through the matching found in section 2.3.3, the duality is realized as conjugation of momenta in the Toda CFT when $\widetilde{N_{f}}=N_{f}$. Theories with $\widetilde{N_{f}}<N_{f}$ are obtained by decoupling chiral multiplets. For $l=1$, integrating out $X$ reproduces the Seiberg duality between SQCD theories.

The electric and magnetic theories are $\mathcal{N}=(2,2)$ SQCDA theories with gauge groups $\mathrm{U}(N)$ and $\mathrm{U}\left(N^{D}\right)$ and the superpotential coupling

$$
W=\operatorname{Tr} X^{l+1} \quad \text { hence } \quad \mathrm{i} m_{X}^{D}=\mathrm{i} m_{X}=\frac{-1}{l+1}
$$

for some integer $l \geq 1$. As we will see, $z^{D}=(-1)^{N_{f}-\widetilde{N}_{f}} z, \bar{z}^{D}=(-1)^{N_{f}-\widetilde{N_{f}}} \bar{z}$ (in terms of renormalized parameters at the scale $\ell$ of the sphere), $N^{D}=l N_{f}-N, m_{t}^{D}=m_{X}-m_{t}$, $\widetilde{m}_{t}^{D}=m_{X}-\widetilde{m}_{t}, m_{X}^{D}=m_{X}$, and the magnetic theory also features $l N_{f} \widetilde{N_{f}}$ free mesons $M_{j s t}^{D}$ with twisted masses $m_{j s t}^{D}=m_{s}+\widetilde{m}_{t}+j m_{X}$ for $0 \leq j<l, 1 \leq s \leq N_{f}, 1 \leq t \leq \widetilde{N_{f}}$. We assume that $l \leq N \leq l N_{f}-l$.

We test the duality by comparing $S^{2}$ partition functions. Namely, we prove that

$$
Z_{S^{2}}^{\mathrm{U}(N), N_{f}, \widetilde{N_{f}}}(m ; z, \bar{z})=a(z, \bar{z}) \prod_{j, s, t} \gamma\left(-\mathrm{i} m_{j s t}^{D}\right) Z_{S^{2}}^{\mathrm{U}\left(N^{D}\right), N_{f}, \widetilde{N}_{f}}\left(m^{D} ; z^{D}, \bar{z}^{D}\right)
$$

with dual parameters given above. The constant factor in (3.39) is the one-loop determinant of free mesons $M_{j s t}^{D}$ whose twisted masses $m_{j s t}^{D}=m_{s}+\widetilde{m}_{t}+j m_{X}$ are fixed by the full superpotential coupling

$$
W=\operatorname{Tr}\left[\left(X^{D}\right)^{l+1}\right]+\sum_{s=1}^{N_{f}} \sum_{t=1}^{\widetilde{N_{f}}} \sum_{j=0}^{l-1} M_{j s t}^{D}\left[\widetilde{q}_{t}^{D}\left(X^{D}\right)^{l-1-j} q_{s}^{D}\right] .
$$

Relative coefficients are unimportant, as the superpotential only affects the $S^{2}$ partition function by fixing complexified twisted masses. The electric theory features mesons $\widetilde{q}_{t} X^{j} q_{s}$ which have the same twisted masses $m_{s}+\widetilde{m}_{t}+j m_{X}$. The factor $a(z, \bar{z})$ is

$$
\begin{aligned}
a(z, \bar{z}) & = \begin{cases}|z|^{2 \delta_{0}} & \text { if } \widetilde{N_{f}} \leq N_{f}-2, \\
|z|^{2 \delta_{0}} e^{l(-1)^{N_{f}(z-\bar{z})}} & \text { if } \widetilde{N_{f}}=N_{f}-1, \\
|z|^{2 \delta_{0}}\left|1-(-1)^{N_{f}} z\right|^{2 \delta_{1}} & \text { if } \widetilde{N_{f}}=N_{f},\end{cases} \\
\delta_{0} & =-\frac{l N_{f}}{2}+\frac{l N}{l+1}-l \sum_{s=1}^{N_{f}} \mathrm{i} m_{s}, \quad \delta_{1}=l N_{f}-\frac{2 l N}{l+1}+l \sum_{s=1}^{N_{f}}\left(\mathrm{i} m_{s}+\mathrm{i} \widetilde{m}_{s}\right) .
\end{aligned}
$$

As discussed below (3.2) for the Seiberg duality, this factor can be absorbed as an ambiguity of the $S^{2}$ partition function.

The same consistency checks as for the Seiberg duality apply. Repeating the duality yields the original parameters, and the factors $a(z, \bar{z})$ and products of gamma functions cancel. Charge conjugation leaves the relation invariant in the case $\widetilde{N_{f}}=N_{f}$. 
Let us first derive (3.39) for $\widetilde{N_{f}}=N_{f}$ from Toda CFT conjugation. Recall (2.111), which expresses the partition functions of interest as $b^{2} \rightarrow \frac{-1}{l+1}$ limits of Toda CFT four-point functions. The relevant correlator is $\left\langle\widehat{V}_{\alpha_{\infty}}(\infty) \widehat{V}_{\hat{m}}(1) \widehat{V}_{-b \omega_{N, l}}(x, \bar{x}) \widehat{V}_{\alpha_{0}}(0)\right\rangle$. Here, $\omega_{N, l}=l \omega_{k}+(N-l k) h_{k+1}$ with $k$ defined by $k l \leq N<(k+1) l$, and its conjugate weight is $\omega_{N, l}^{C}=\omega_{N^{D}, l}$ with $N^{D}=l N_{f}-N$. As for the Seiberg duality, we follow the conjugation of $\hat{m}=(\varkappa+N b) h_{1}$ by a Weyl reflection to get a momentum along $h_{1}$,

$$
\hat{m}^{D}=\left(\varkappa^{D}+N^{D} b\right) h_{1}=\left[N_{f}\left(b+\frac{1}{b}\right)-\varkappa-N b\right] h_{1} .
$$

Thus, $\varkappa^{D}=\frac{1}{b} N_{f}\left(1-(l-1) b^{2}\right)-\varkappa$, which is $\frac{2}{b} N_{f}\left(1+b^{2}\right)-\varkappa$ when $b^{2}=\frac{-1}{l+1}$. Finally, the generic momenta $\alpha_{0}$ and $\alpha_{\infty}$ are mapped as $\alpha \rightarrow 2 Q-\alpha$ under a conjugation followed by the maximal Weyl reflection. Translating to gauge theory parameters thanks to the dictionary (2.89) yields the values of $N^{D}, m_{X}^{D}, m_{s}^{D}$, and $\widetilde{m}_{s}^{D}$ claimed earlier. The position $y=(-1)^{N_{f}} z$ of the degenerate operator is not affected by conjugation, hence $y^{D}=y$ and $z^{D}=z$. The factor $a(z, \bar{z})$ given in (3.41) is the ratio of factors $|y|^{2 \gamma_{0}}|1-y|^{2 \gamma_{1}}$ for the electric and magnetic theories.

Since the constant factor $A$ which appears in the matching (2.111) is not known, we cannot deduce the presence of free mesons in the magnetic theory through conjugation of momenta. Instead, we use the Higgs branch decomposition (2.95), which expresses both partition functions as sums over choices of $0 \leq n_{s} \leq l$ with $n_{1}+\cdots+n_{N_{f}}=N$. The classical contribution for the term labeled by $\left\{n_{s}\right\}$ in the electric theory is $|z|^{2 \delta_{0}}$ times the classical contribution for the term labeled by $\left\{n_{s}^{D}=l-n_{s}\right\}$ in the magnetic theory. We then compare the one-loop determinants (2.96) for those vacua,

$$
\begin{aligned}
\frac{Z_{11,\{n\}}}{Z_{11,\{l-n\}}^{D}} & =\prod_{s=1}^{N_{f}} \prod_{t=1}^{N_{f}}\left[\prod_{\mu=0}^{n_{s}-1} \frac{\gamma\left(-\mathrm{i} m_{t}+\mathrm{i} m_{s}+\frac{n_{t}-\mu}{l+1}\right)}{\gamma\left(1+\mathrm{i} \widetilde{m}_{t}+\mathrm{i} m_{s}-\frac{\mu}{l+1}\right)} \prod_{\mu=0}^{l-n_{s}-1} \frac{\gamma\left(1+\mathrm{i} \widetilde{m}_{t}^{D}+\mathrm{i} m_{s}^{D}-\frac{\mu}{l+1}\right)}{\gamma\left(-\mathrm{i} m_{t}^{D}+\mathrm{i} m_{s}^{D}+\frac{n_{t}^{D}-\mu}{l+1}\right)}\right] \\
& =\prod_{s=1}^{N_{f}} \prod_{t=1}^{N_{f}} \prod_{j=0}^{l-1} \gamma\left(-\mathrm{i} \widetilde{m}_{t}-\mathrm{i} m_{s}+\frac{j}{l+1}\right),
\end{aligned}
$$

and find the one-loop determinants of mesons with twisted masses $m_{j s t}^{D}$ as announced. Finally, we prove in appendix B.2 that vortex partition functions of dual theories are equal up to the factor $(1-y)^{\delta_{1}}$, hence establishing the relation (3.39) for $\widetilde{N_{f}}=N_{f}$.

For completeness, we compare exponents which appear when expanding the $S^{2}$ partition functions of the dual theories near $y=1$. Those exponents are given by (2.100): for a general $\mathcal{N}=(2,2)$ SQCDA theory with $N$ colors there are $N+1$ exponents labeled by an integer $0 \leq k \leq N$. The set of exponents thus does not match for the $\mathrm{U}(N)$ and $\mathrm{U}\left(N^{D}\right)$ theories we consider here. In fact, it turns out that only the subset labeled by $0 \leq k \leq l$ matches (assuming that $l \leq N \leq l N_{f}-l$ ): the coefficients of all other exponents must thus vanish when $\mathrm{i} m_{X}=\frac{-1}{l+1}$.

We now take the limit i $\widetilde{m}_{t}=\mathrm{i} \Lambda \rightarrow \pm \mathrm{i} \infty$ for $N_{f}-\widetilde{N_{f}}$ antifundamental flavours $\widetilde{N}_{f}<$ $t \leq N_{f}$. The multiplets $\widetilde{q}_{t}$ decouple, the FI parameter is renormalized, and we will be left with the Kutasov-Schwimmer duality (3.39) for $\widetilde{N}_{f}<N_{f}$. 
The twisted mass $\Lambda$ appears in the Coulomb branch expansion (2.93) through the one-loop determinant of antifundamental chiral multiplets: for fixed $\sigma$ and $B$

$$
\prod_{j=1}^{N} \prod_{t=\widetilde{N}_{f}+1}^{N_{f}} \frac{\Gamma\left(-\mathrm{i} \widetilde{m}_{t}+\mathrm{i} \sigma_{j}+\frac{B_{j}}{2}\right)}{\Gamma\left(1+\mathrm{i} \widetilde{m}_{t}-\mathrm{i} \sigma_{j}+\frac{B_{j}}{2}\right)} \sim\left[\gamma(-\mathrm{i} \Lambda)^{N}(-\mathrm{i} \Lambda)^{\operatorname{Tr}\left(\mathrm{i} \sigma+\frac{B}{2}\right)}(\mathrm{i} \Lambda)^{\operatorname{Tr}\left(\mathrm{i} \sigma-\frac{B}{2}\right)}\right]^{N_{f}-\widetilde{N}_{f}} .
$$

The powers of $\pm \mathrm{i} \Lambda$ combine with the classical contribution $z_{\text {bare }}^{\operatorname{Tr}(\mathrm{i} \sigma+B / 2)} \bar{z}_{\mathrm{bare}}^{\operatorname{Tr}(\mathrm{i} \sigma-B / 2)}$, and we get the integrand of the Coulomb branch representation for the theory with $\widetilde{N}_{f}<N_{f}$ and $z=z_{\text {ren }}=(-\mathrm{i} \Lambda)^{N_{f}-\widetilde{N}_{f}} z_{\text {bare }}$. A careful treatment shows that the limit $\Lambda \rightarrow \pm \infty$ and the integration commute, because the contribution for large values of $\sigma$ and $B$ falls off fast enough at infinity. As mentioned for a similar limit of the SQCD theory in section 2.2.3, it is easier to work out this convergence issue in the Higgs branch decomposition where terms decrease exponentially in the vorticity $k$. Either way yields

$$
\begin{aligned}
Z_{S^{2}}^{N_{f}, N_{f}}\left(\frac{z}{(-\mathrm{i} \Lambda)^{N_{f}-\widetilde{N_{f}}}}, \frac{\bar{z}}{\left.(\mathrm{i} \Lambda)^{N_{f}-\widetilde{N_{f}}},\left\{m_{s}\right\},\left\{\widetilde{m}_{s}, \Lambda\right\}\right)}\right. \\
\sim \gamma(-\mathrm{i} \Lambda)^{N\left(N_{f}-\widetilde{N_{f}}\right)} Z_{S^{2}}^{N_{f}, \widetilde{N_{f}}}\left(\left\{m_{s}\right\},\left\{\widetilde{m}_{s}\right\}, z, \bar{z}\right) .
\end{aligned}
$$

Given the form of (3.46), the next step is to consider the duality (3.39) with the replacement $\widetilde{N_{f}} \rightarrow N_{f}, z \rightarrow z /(-\mathrm{i} \Lambda)^{N_{f}-\widetilde{N_{f}}}$ and $\bar{z} \rightarrow \bar{z} /(\mathrm{i} \Lambda)^{N_{f}-\widetilde{N_{f}}}$, in the limit where $\Lambda \rightarrow \pm \infty$. The factor $a(z, \bar{z})=|z|^{2 \delta_{0}}\left|1-(-1)^{N_{f}} z\right|^{2 \delta_{1}}$ with $\delta_{1} \sim \mathrm{i} \Lambda l$ becomes

$$
\begin{aligned}
a_{N_{f}, N_{f}}\left(\frac{z}{(-\mathrm{i} \Lambda)^{N_{f}-\widetilde{N_{f}}}}, \frac{\bar{z}}{(\mathrm{i} \Lambda)^{N_{f}-\widetilde{N_{f}}}}\right) & \sim \Lambda^{-2\left(N_{f}-\widetilde{N_{f}}\right) \delta_{0}} a_{N_{f}, \widetilde{N_{f}}}(z, \bar{z}) \\
& \sim \begin{cases}\Lambda^{-2\left(N_{f}-\widetilde{N_{f}}\right) \delta_{0}}|z|^{2 \delta_{0}} & \text { if } \widetilde{N_{f}} \leq N_{f}-2, \\
\Lambda^{-2\left(N_{f}-\widetilde{N_{f}}\right) \delta_{0}}|z|^{2 \delta_{0}} e^{l(-1)^{N_{f}(z-\bar{z})}} & \text { if } \widetilde{N_{f}}=N_{f}-1 .\end{cases}
\end{aligned}
$$

The gamma functions in (3.39) become those for $\widetilde{N_{f}}<N_{f}$, multiplied by

$$
\begin{aligned}
\prod_{s=1}^{N_{f}} \prod_{j=0}^{l-1} \prod_{t=\widetilde{N}_{f}+1}^{N_{f}} \gamma\left(-\mathrm{i} \Lambda-\mathrm{i} m_{s}+\frac{j}{l+1}\right) & \sim \prod_{s=1}^{N_{f}} \prod_{j=0}^{l-1}\left[\gamma(-\mathrm{i} \Lambda) \Lambda^{2\left(-\mathrm{i} m_{s}+\frac{j}{l+1}\right)}\right]^{N_{f}-\widetilde{N_{f}}} \\
& \sim\left[\gamma(-\mathrm{i} \Lambda)^{l N_{f}} \Lambda^{2\left(\delta_{0}+N^{D} \frac{l}{l+1}\right)}\right]^{N_{f}-\widetilde{N}_{f}} \\
& \sim\left[\Lambda^{2 \delta_{0}} \gamma(-\mathrm{i} \Lambda)^{N} \gamma\left(-\mathrm{i} \Lambda^{D}\right)^{-N^{D}}\right]^{N_{f}-\widetilde{N_{f}}}
\end{aligned}
$$

where we used $\gamma(\mathrm{i} x+a) \sim \gamma(\mathrm{i} x)|x|^{2 a}$ as $x \rightarrow \pm \infty$, and $\Lambda^{\frac{2 l}{l+1}} \gamma(-\mathrm{i} \Lambda) \sim \gamma\left(-\mathrm{i} \Lambda^{D}\right)^{-1}$. Combining (3.47) and (3.48) with the power of $\gamma(-\mathrm{i} \Lambda)$ from (3.46) and the power of $\gamma\left(-\mathrm{i} \Lambda^{D}\right)$ for the dual theory establishes the Kutasov-Schwimmer duality relation (3.39) for all $\widetilde{N}_{f} \leq N_{f}$.

\subsection{Dualities for quivers}

We revisit here the $\mathcal{N}=(2,2)$ quivers of section 2.4 and express some Seiberg and $\mathcal{N}=$ $(2,2)^{*}$ dualities as permutations of Toda CFT punctures in section 3.3.1. This lets us 
construct in section 3.3.2 the full set of theories obtained through Seiberg and $\mathcal{N}=(2,2)^{*}$ dualities. For a particular choice of matter content, a certain combination of dualities is realized as conjugation of momenta in the Toda CFT.

The gauge theories depend on ranks $N_{j} \geq 0$, signs $\eta_{j}$, and complexified FI parameters $\left(\hat{z}_{j}, \overline{\hat{z}_{j}}\right)$ for $1 \leq j \leq n$, as well as twisted masses. They are described by the quiver

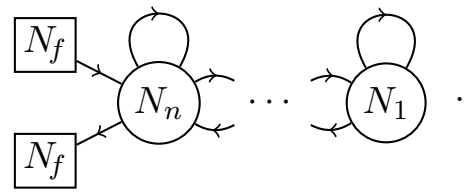

The theories consist of a $\mathrm{U}\left(N_{1}\right) \times \cdots \times \mathrm{U}\left(N_{n}\right)$ vector multiplet coupled to chiral multiplets which transform in the following representations: $N_{f}$ fundamentals and $N_{f}$ antifundamentals of $\mathrm{U}\left(N_{n}\right)$, two bifundamentals of $\mathrm{U}\left(N_{j}\right) \times \mathrm{U}\left(N_{j-1}\right)$ for each $2 \leq j \leq n$, and one adjoint of $\mathrm{U}\left(N_{j}\right)$ for each $1 \leq j \leq n$. Let $\epsilon_{j}=\prod_{i=j}^{n} \eta_{i}$. The twisted masses $m_{t}, \widetilde{m}_{t}, m_{j(j-1)}$, $m_{(j-1) j}$, and $m_{j j}$ of those chiral multiplets obey (2.118), that is,

$$
\mathrm{i} m_{j(j-1)}+\mathrm{i} m_{(j-1) j}=-1-2 q_{j} \quad \text { and } \quad \mathrm{i} m_{j j}=q_{j}+q_{j+1},
$$

where $q_{j}=b^{2} / 2$ if $\epsilon_{j}=1$ and $q_{j}=-\left(1+b^{2}\right) / 2$ otherwise for some parameter $b^{2}$. The twisted masses are such that a given superpotential $W_{\eta}$ has $R$-charge 2 (twisted mass i). Whenever $\eta_{j}=-1$, the superpotential term $\operatorname{Tr}\left(X_{j}^{2}\right)$ lets us integrate out $X_{j}$.

We gave evidence in section 2.4 that the partition function on $S_{b}^{4}$ of the $S^{2}$ surface operator obtained by coupling such a theory to free hypermultiplets is equal to a Toda CFT $(n+3)$-point function, namely the correlator of two generic, one semi-degenerate, and $n$ degenerate vertex operators. The momenta of the first three operators encode the twisted masses $m_{t}$ and $\widetilde{m}_{t}$. The degenerate operators are inserted at positions $x_{j}=\prod_{i=j}^{n} \hat{z}_{i}$, and have momenta $-b \Omega_{j}=-b \Omega\left(K_{j}, \epsilon_{j}\right)$, where $K_{j}=N_{j}-N_{j-1}, \epsilon_{j}=\prod_{i=j}^{n} \eta_{i}$, and $\Omega(K,+1)=K h_{1}$ and $\Omega(K,-1)=\omega_{K}$.

Crossing symmetry of the Toda CFT correlator states that the labeling of degenerate operators by integers $1 \leq j \leq n$ is irrelevant. Therefore, the $n$ ! gauge theories which correspond to each labeling of the degenerate punctures should all have identical $S^{2}$ partition functions, up to simple factors. It turns out that each transposition $k \leftrightarrow k+1$ (for $k<n$ ) corresponds to a duality acting on the node $\mathrm{U}\left(N_{k}\right)$ of the quiver gauge theory: Seiberg duality (see section 3.1) if $\eta_{k}=-1$, or the $\mathcal{N}=(2,2)^{*}$ duality (see section 3.2.1) if $\eta_{k}=+1$. In section 3.3.1 we work out details and make sure that transpositions correctly reproduce the mapping of parameters for such dualities. As a result, the groupoid generated by Seiberg and $\mathcal{N}=(2,2)^{*}$ dualities acting on nodes with $k<n$ is realized as permutations of punctures in the Toda CFT.

In section 3.3.2, we extend the groupoid by including the action of Seiberg duality on the node $\mathrm{U}\left(N_{n}\right)$ when it is applicable $\left(\eta_{n}=-1\right)$ : the $\mathcal{N}=(2,2)^{*}$ duality never applies, since the $N_{f}$ fundamental and antifundamental chiral multiplets are not constrained by a superpotential. The result of acting with Seiberg duality on $\mathrm{U}\left(N_{n}\right)$ is not a quiver of the same type, hence is not given a Toda CFT interpretation in our work. However, for a specific choice of matter content which corresponds to the case where all degenerate vertex 
operators are labeled by antisymmetric representations of $A_{N_{f}-1}$, applying Seiberg duality in turn to all the nodes yields a quiver of the original form. This combination of dualities corresponds to conjugating Toda CFT momenta.

All our results extend to theories with any number $\widetilde{N_{f}} \leq N_{f}$ of antifundamental chiral multiplets following the discussion for Seiberg duality of SQCD in section 3.1.2. We focus on $\widetilde{N}_{f}=N_{f}$ because the matching between partition functions and Toda CFT correlators was only derived in this case in section 2.4: for $\widetilde{N_{f}}<N_{f}$, the correlator contains an irregular puncture as described for SQCD in section 2.2.3.

\subsubsection{Seiberg dualities from braiding moves}

We now prove that the action of Seiberg duality or the $\mathcal{N}=(2,2)^{*}$ duality (depending on $\left.\eta_{k}\right)$ on the node $\mathrm{U}\left(N_{k}\right)$ translates to the transposition $\left(x_{k}, \epsilon_{k}, K_{k}\right) \leftrightarrow\left(x_{k+1}, \epsilon_{k+1}, K_{k+1}\right)$ of two degenerate punctures, for $1 \leq k \leq n-1$. Specifically, we show that the $S^{2}$ partition functions of the theories described by the Toda CFT data before and after the transposition are equal. Most gauge theory parameters describing the electric and magnetic theories are the same, with the following changes: $\eta_{k \pm 1}^{D}=\eta_{k \pm 1} \eta_{k}, N_{k}^{D}=N_{k+1}+N_{k-1}-N_{k}$, $\hat{z}_{k \pm 1}^{D}=\hat{z}_{k \pm 1} \hat{z}_{k}$ and $\hat{z}_{k}^{D}=\hat{z}_{k}^{-1}$.

The multiplets which interact with the $\mathrm{U}\left(N_{k}\right)$ vector multiplet of the electric theory are those of $\mathcal{N}=(2,2)$ SQCDA with $N_{k}$ colors and $N_{k-1}+N_{k+1}$ flavours. If $\eta_{k}=-1$, then $\mathrm{i} m_{k k}=-1 / 2$ and $W_{\eta}$ contains the term $\operatorname{Tr}\left(X_{k}^{2}\right)$ which lets us integrate out the adjoint chiral multiplet $X_{k}$, leaving $\mathcal{N}=(2,2) \mathrm{SQCD}$. If instead $\eta_{k}=+1$, then $\mathrm{i} m_{k k}+\mathrm{i} m_{k(k \pm 1)}+$ $\mathrm{im}_{(k \pm 1) k}=-1$ and $W_{\eta}$ contains the terms $\operatorname{Tr}\left(\phi_{(k \pm 1) k} X_{k} \phi_{k(k \pm 1)}\right)$ : this is $\mathcal{N}=(2,2)^{*}$ SQCD. In both settings, the theory admits a dual description with $N_{k+1}+N_{k-1}-N_{k}$ colors, and some mesons if $\eta_{k}=-1$ (see sections 3.1 and 3.2.1). As we will now see, parameters map precisely as expected from the Toda CFT.

In the Coulomb branch representation of the $S^{2}$ partition function of the electric theory, we collect all factors which depend on the scalar $\sigma_{k}$ of the $\mathrm{U}\left(N_{k}\right)$ vector multiplet. This yields an integral $Z_{k}(2.132)$ very similar to the partition function of $\mathcal{N}=(2,2)$ SQCDA with $N_{k}$ colors and $N_{k-1}+N_{k+1}$ flavours. The usual contour techniques apply and yield a factorized expression for $Z_{k}$ in the region $\left|\hat{z}_{k}\right|<1$, namely

$$
\begin{aligned}
Z_{k}=\sum_{\text {Higgs vacuum } v^{ \pm}} & \hat{z}_{k}^{\operatorname{Tr} i v^{+}} \overline{\hat{z}}_{k}^{\operatorname{Tr} i v^{-}} Z_{11,\left\{v^{ \pm}\right\}}\left(\left\{m_{k l}-\sigma_{l j}^{ \pm}\right\},\left\{m_{l k}+\sigma_{l j}^{ \pm}\right\}\right) \\
& Z_{\mathrm{v}, v^{+}}\left(\left\{m_{k l}-\sigma_{l j}^{+}\right\},\left\{m_{l k}+\sigma_{l j}^{+}\right\} ; \hat{z}_{k}\right) \\
& \cdot Z_{\overline{\mathrm{v}}, v^{-}}\left(\left\{m_{k l}-\sigma_{l j}^{-}\right\},\left\{m_{l k}+\sigma_{l j}^{-}\right\} ; \overline{\hat{z}}_{k}\right) .
\end{aligned}
$$

As discussed above, the superpotential $W_{\eta}$ reduces SQCDA to $\mathcal{N}=(2,2) \operatorname{SQCD}$ or $\mathcal{N}=$ $(2,2)^{*}$ SQCD depending on $\eta_{k}$. In both cases, Higgs branch vacua are labeled by sets of $N_{k}$ "flavours" among

$$
L_{k}=\left\{(l, j) \mid l=k \pm 1,1 \leq j \leq N_{l}\right\}
$$

and the eigenvalues of $v^{ \pm}$for a given $N_{k}$-element subset $E \subset L_{k}$ are

$$
v_{(l, j)}^{ \pm}=-m_{k l}+\sigma_{l j}^{ \pm} \quad \text { for }(l, j) \in E .
$$


The vortex partition functions in (3.51) are those of the relevant $\mathcal{N}=(2,2)$ SQCD or $\mathcal{N}=$ $(2,2)^{*}$ SQCD theory with $N_{k+1}+N_{k-1}$ fundamental multiplets of twisted masses $\left\{m_{k l}-\sigma_{l j}^{+}\right\}$ and the same number of antifundamental multiplets of twisted masses $\left\{m_{l k}+\sigma_{l j}^{+}\right\}$, in the Higgs branch vacuum $v^{+}$. The antivortex partition functions are obtained by replacing $\sigma_{l j}^{+}$ by $\sigma_{l j}^{-}$and $v^{+}$by $v^{-}$. The one-loop determinant for the vacuum labeled by $E \subset L_{k}$ is

$$
\begin{array}{r}
Z_{11, E}=\prod_{(l, j) \in E} \prod_{\left(l^{\prime}, j^{\prime}\right) \in L_{k}}\left[\frac{\Gamma\left(-\mathrm{i} m_{k l^{\prime}}-\delta_{l^{\prime} j^{\prime} \in E} \mathrm{i} m_{k k}+\mathrm{i} m_{k l}+\mathrm{i} \sigma_{l^{\prime} j^{\prime}}^{+}-\mathrm{i} \sigma_{l j}^{+}\right)}{\Gamma\left(1+\mathrm{i} m_{k l^{\prime}}+\delta_{l^{\prime} j^{\prime} \in E} \mathrm{i} m_{k k}-\mathrm{i} m_{k l}-\mathrm{i} \sigma_{l^{\prime} j^{\prime}}^{-}+\mathrm{i} \sigma_{l j}^{-}\right)}\right. \\
\left.\cdot \frac{\Gamma\left(-\mathrm{i} m_{l^{\prime} k}-\mathrm{i} m_{k l}-\mathrm{i} \sigma_{l^{\prime} j^{\prime}}^{+}+\mathrm{i} \sigma_{l j}^{+}\right)}{\Gamma\left(1+\mathrm{i} m_{l^{\prime} k}+\mathrm{i} m_{k l}+\mathrm{i} \sigma_{l^{\prime} j^{\prime}}^{-}-\mathrm{i} \sigma_{l j}^{-}\right)}\right]
\end{array}
$$

We now need to distinguish $\eta_{k}= \pm 1$ because explicit expressions differ. We will bring the results together at the end of this section.

Focus first on the case $\eta_{k}=+1$. Since $1+\mathrm{i} m_{k k}+\mathrm{i} m_{k(k \pm 1)}+\mathrm{i} m_{(k \pm 1) k}=0$, the factors with $(l, j) \in E$ and $\left(l^{\prime}, j^{\prime}\right) \in E$ in $(3.54)$ cancel. The remaining factors are invariant under the exchanges $E \rightarrow E^{\complement}$ and $m_{k l}-\sigma_{l j}^{ \pm} \leftrightarrow m_{l k}+\sigma_{l j}^{ \pm}$. As a result, the one-loop determinant for the s-channel vacuum $E$ of the $\mathrm{U}\left(N_{k}\right)$ theory is equal to the one-loop determinant for the u-channel vacuum $E^{\complement}$ of a theory with identical twisted masses but $N_{k}^{D}=\# E^{\complement}=N_{k-1}+N_{k+1}-N_{k}$ colors. As discussed in section 3.2.1 and shown directly in appendix B.2, the vortex partition functions of the $\mathrm{U}\left(N_{k}\right)$ theory in the s-channel vacuum $E$ and of the $\mathrm{U}\left(N_{k}^{D}\right)$ theory in the u-channel vacuum $E^{\complement}$ are equal up to a factor (B.57)

$$
Z_{\mathrm{v}, E}^{\mathrm{U}\left(N_{k}\right)}\left(\hat{z}_{k}\right)=\left(1-\hat{z}_{k}\right)^{-\delta_{1}} Z_{\mathrm{v}, E^{\mathrm{C}}}^{\mathrm{U}\left(N_{k}^{D}\right)}\left(\left(\hat{z}_{k}^{D}\right)^{-1}\right)
$$

with $\delta_{1}=\left(N_{k}-N_{k}^{D}\right)\left(1+\mathrm{i} m_{k k}\right)$, provided $\hat{z}_{k}^{D}=\hat{z}_{k}^{-1}$ as expected from the Toda CFT symmetry. Finally, the classical contribution transforms as follows:

$$
\prod_{(l, j) \in E} \hat{z}_{k}^{-\mathrm{i} m_{k l}+\mathrm{i} \sigma_{l j}^{+}}=\hat{z}_{k}^{-\delta_{0}+\operatorname{Tr} \mathrm{i} \sigma_{k-1}^{+}+\operatorname{Tr} \mathrm{i} \sigma_{k+1}^{+}} \prod_{(l, j) \in E^{\mathrm{C}}}\left(\hat{z}_{k}^{D}\right)^{\mathrm{i} m_{l k}+\mathrm{i} \sigma_{l j}^{+}}
$$

with $\delta_{0}=N_{k-1} \mathrm{i} m_{k(k-1)}+N_{k+1} \mathrm{i} m_{k(k+1)}+\left(1+\mathrm{i} m_{k k}\right) N_{k}^{D}$. All in all,

$$
Z_{k}^{\mathrm{U}\left(N_{k}\right)}\left(z_{k}, \bar{z}_{k}\right)=\left|\hat{z}_{k}\right|^{-2 \delta_{0}}\left|1-\hat{z}_{k}\right|^{-2 \delta_{1}} \hat{z}_{k}^{\operatorname{Tr} \mathrm{i} \sigma_{k-1}^{+}+\operatorname{Tr} \mathrm{i} \sigma_{k+1}^{+}} \overline{\hat{z}}_{k}^{\operatorname{Tr} \mathrm{i} \sigma_{k-1}^{-}+\operatorname{Tr} \mathrm{i} \sigma_{k+1}^{-}} Z_{k}^{\mathrm{U}\left(N_{k}^{D}\right)}\left(z_{k}^{D}, \bar{z}_{k}^{D}\right) .
$$

In the full $S^{2}$ partition function of the quiver theory, the powers of $\hat{z}_{k}$ and $\overline{\hat{z}}_{k}$ combine with the classical contribution for the gauge group factors $\mathrm{U}\left(N_{k \pm 1}\right)$ and yield

$$
\left|\hat{z}_{k}\right|^{-2 \delta_{0}}\left|1-\hat{z}_{k}\right|^{-2 \delta_{1}} \prod_{l=k \pm 1}\left(\hat{z}_{l} \hat{z}_{k}\right)^{\operatorname{Tr} \mathrm{i} \sigma_{l}^{+}}\left(\overline{\hat{z}_{l} \hat{z}_{k}}\right)^{\operatorname{Tr} \mathrm{i} \sigma_{l}^{-}}
$$

Therefore, the $S^{2}$ partition functions of the $\mathrm{U}\left(N_{1}\right) \times \cdots \times \mathrm{U}\left(N_{n}\right)$ theory and of the theory with $N_{k}^{D}=N_{k-1}+N_{k+1}-N_{k}, \hat{z}_{k}^{D}=\hat{z}_{k}^{-1}$, and $\hat{z}_{k \pm 1}^{D}=\hat{z}_{k \pm 1} \hat{z}_{k}$ are equal up to $\left|\hat{z}_{k}\right|^{-2 \delta_{0}} \mid 1-$ $\left.\hat{z}_{k}\right|^{-2 \delta_{1}}$. On the Toda CFT side, this factor is due to differences in powers of $\left|x_{k}\right|^{2},\left|x_{k+1}\right|^{2}$ and $\left|x_{k+1}-x_{k}\right|^{2}$ which appear in the correspondences for the electric and magnetic theories. 
In gauge theory, the factor can be absorbed into the partition function: since $\delta_{1}$ only depends on $b$, the $N_{j}$, and the matter content, $\left|1-\hat{z}_{k}\right|^{-2 \delta_{1}}$ is a renormalization scheme ambiguity, while $\left|\hat{z}_{k}\right|^{-2 \delta_{0}}$ can be absorbed by a global $\mathrm{U}(1) \subset \mathrm{U}\left(N_{k}\right)$ gauge transformation. Such ambiguities are described below (2.10).

The case $\eta_{k}=-1$ follows the same ideas, but is more intricate. The Higgs branch decomposition (3.54) involves vortex partition functions of $\mathcal{N}=(2,2)$ SQCD. As for the previous case, those are equal up to a power of $\left(1-\hat{z}_{k}\right)$ to vortex partition functions of a dual theory with $N_{k}^{D}$ colors and twisted masses $m^{D}=\mathrm{i} / 2-m$. Explicitly,

$$
\begin{aligned}
& Z_{\mathrm{v}, E}\left(\left\{m_{k l}-\sigma_{l j}^{+}\right\},\left\{m_{l k}+\sigma_{l j}^{+}\right\} ; \hat{z}_{k}\right) \\
& \quad=\left(1-\hat{z}_{k}\right)^{-N_{k}-2 q_{k} N_{k-1}-2 q_{k+1} N_{k+1}} Z_{\mathrm{v}, E^{\mathrm{C}}}\left(\left\{\mathrm{i} / 2-m_{k l}+\sigma_{l j}^{+}\right\},\left\{\mathrm{i} / 2-m_{l k}-\sigma_{l j}^{+}\right\} ; \hat{z}_{k}\right) .
\end{aligned}
$$

The signs with which $\sigma_{l j}^{+}$appears in the right-hand side are inconvenient, as it implies that chiral multiplets which transform under the fundamental representation of $\mathrm{U}\left(N_{k}^{D}\right)$ also transform in the fundamental representation of $\mathrm{U}\left(N_{l}^{D}\right)$, and not the antifundamental representation. This is fixed by conjugating all $\mathrm{U}\left(N_{k}^{D}\right)$ charges: $\hat{z}_{k} \rightarrow \hat{z}_{k}^{-1}$ and the vortex partition function becomes a u-channel $\left(\left|\hat{z}_{k}^{-1}\right| \rightarrow \infty\right)$ vortex partition function of SQCD with $N_{k}^{D}$ colors, $N_{k-1}+N_{k+1}$ flavours, and $\hat{z}_{k}^{D}=\hat{z}_{k}^{-1}$. Once this is understood, the classical contributions (of the electric s-channel vacuum labeled by $E$ and the magnetic u-channel vacuum labeled by $E^{\complement}$ ) are equal up to powers of $\left|\hat{z}_{k}\right|^{2}$, provided $\hat{z}_{k}^{D}=\hat{z}_{k}^{-1}$ and $\hat{z}_{k \pm 1}^{D}=\hat{z}_{k \pm 1} \hat{z}_{k}$. This is precisely the map described by the exchange of Toda CFT punctures.

The one-loop determinants, on the other hand, transform non-trivially. This is expected from the study of Seiberg duality for $\mathcal{N}=(2,2)$ SQCD: the magnetic theory includes mesons whose one-loop determinants appear in the $S^{2}$ partition function. There, the mesons are realized as $M_{t s}=\widetilde{q}_{t} q_{s}$ in terms of the electric quarks and antiquarks $q_{s}$ and $\widetilde{q}$, and couple to the magnetic multiplets through a superpotential term $\widetilde{q}_{t}^{D} M_{t s} q_{s}^{D}$. In our current setting, the mesons are the four combinations $M_{l l^{\prime}}=\phi_{l k} \phi_{k l^{\prime}}$ in the electric theory, and couple to the magnetic multiplets through the superpotential $\operatorname{Tr}\left(M_{l l^{\prime}} \phi_{l^{\prime} k}^{D} \phi_{k l}^{D}\right)$. The mesons $M_{(k \pm 1)(k \pm 1)}$ transform in the adjoint representations of $\mathrm{U}\left(N_{k \pm 1}\right)$, and the mesons $M_{(k \pm 1)(k \mp 1)}$ in bifundamental representations of $\mathrm{U}\left(N_{k+1}\right) \times \mathrm{U}\left(N_{k-1}\right)$. Since the (electric) superpotential features the term $\operatorname{Tr}\left(M_{(k-1)(k+1)} M_{(k+1)(k-1)}\right)$ for $\eta_{k}=-1$, these two mesons can be integrated out, leaving the term $\operatorname{Tr}\left(\phi_{(k-1) k}^{D} \phi_{k(k+1)}^{D} \phi_{(k+1) k}^{D} \phi_{k(k-1)}^{D}\right)$ in the superpotential of the magnetic theory. This term is expected from $\eta_{k}^{D}=-1$.

Next, for each of $l=k \pm 1$ there are two cases. If $\eta_{k \pm 1}=+1$ then the superpotential term $\operatorname{Tr}\left(X_{k \pm 1} M_{(k \pm 1)(k \pm 1)}\right)$ lets us integrate out both $X_{k \pm 1}$ and the meson $M_{(k \pm 1)(k \pm 1)}$, leaving the magnetic superpotential $\operatorname{Tr}\left(\phi_{(k \pm 1)(k \pm 2)} \phi_{(k \pm 2)(k \pm 1)} \phi_{(k \pm 1) k}^{D} \phi_{k(k \pm 1)}^{D}\right)$. This is expected from $\eta_{k \pm 1}^{D}=-1$ (multiplets $\phi_{l l^{\prime}}$ with $l, l^{\prime} \neq k$ are not affected by the duality). If instead $\eta_{k \pm 1}=-1$, then we integrate out $X_{k \pm 1}$, and note the presence of magnetic superpotential terms $\operatorname{Tr}\left(\phi_{(k \pm 1)(k \pm 2)} \phi_{(k \pm 2)(k \pm 1)} M_{(k \pm 1)(k \pm 1)}\right)$ and $\operatorname{Tr}\left(M_{(k \pm 1)(k \pm 1)} \phi_{(k \pm 1) k}^{D} \phi_{k(k \pm 1)}^{D}\right)$. Those are expected from $\eta_{k \pm 1}^{D}=+1$. In both cases, the change in matter content between the electric and magnetic theories and the mapping of twisted masses are encoded in the map $\eta_{k \pm 1}^{D}=\eta_{k \pm 1} \eta_{k}$ implied by the exchange $\epsilon_{k-1} \leftrightarrow \epsilon_{k}$. 
Combining the classical, one-loop, and vortex contributions yields the equality of $S^{2}$ partition functions up to powers of $\left|\hat{z}_{k}\right|^{2}$ and $\left|1-\hat{z}_{k}\right|^{2}$ when $\eta_{k}=-1$. As for $\eta_{k}=+1$, the powers of $\left|1-\hat{z}_{k}\right|^{2}$ and $\left|\hat{z}_{k}\right|^{2}$ are an ambiguity of the $S^{2}$ partition function. This concludes the proof (for arbitrary $\eta$ ) that applying Seiberg duality or the $\mathcal{N}=(2,2)^{*}$ duality to the gauge group factor $\mathrm{U}\left(N_{k}\right)$ with $1 \leq k<n$ corresponds to transposing the punctures $k$ and $k+1$ in the Toda CFT correlator. Therefore, permutations of Toda CFT degenerate punctures encapsulate the mapping of parameters for arbitrary combinations of dualities which act on the nodes with $1 \leq k<n$.

\subsubsection{Seiberg dualities from momentum conjugation}

We now find all theories obtained through dualities acting on any node, including $\mathrm{U}\left(N_{n}\right)$.

For simplicity, we first consider the theory with $\eta_{n}=-1$ and $\eta_{k}=+1$ for $k<n$, which corresponds to a Toda CFT correlator where all degenerate punctures are labeled by antisymmetric representations of $A_{N_{f}-1}\left(\right.$ all $\left.\epsilon_{k}=-1\right)$. Since $\eta_{n}=-1$, the superpotential includes a term $\operatorname{Tr} X_{n}^{2}$ which lets us integrate out the adjoint chiral multiplet $X_{n}$. Therefore, the chiral multiplets which couple to the $\mathrm{U}\left(N_{n}\right)$ vector multiplet are those of $\mathcal{N}=(2,2)$ SQCD with $N_{n}$ colors and $N_{f}+N_{n-1}$ flavours. Applying Seiberg duality and charge conjugation to the node $\mathrm{U}\left(N_{n}\right)$ yields a similar quiver gauge theory with $N_{n}$ replaced by $N_{n}^{D}=N_{f}+N_{n-1}-N_{n}$. Recall that the Seiberg dual of a theory includes additional multiplets with charges identical to mesons of the original theory. Here, these are $N_{f}^{2}$ free chiral multiplets, and $N_{f}$ fundamental, $N_{f}$ antifundamental, and one adjoint of $\mathrm{U}\left(N_{n-1}\right)$. The magnetic theory thus has two adjoints of $\mathrm{U}\left(N_{n-1}\right)$. Given the cubic superpotential which links the bifundamentals of $\mathrm{U}\left(N_{n}\right) \times \mathrm{U}\left(N_{n-1}\right)$ and the adjoint of $\mathrm{U}\left(N_{n-1}\right)$ in the electric theory, the two adjoints of $\mathrm{U}\left(N_{n-1}\right)$ couple through a quadratic superpotential term and can thus be integrated out. Therefore, the two dual theories are given by the quivers

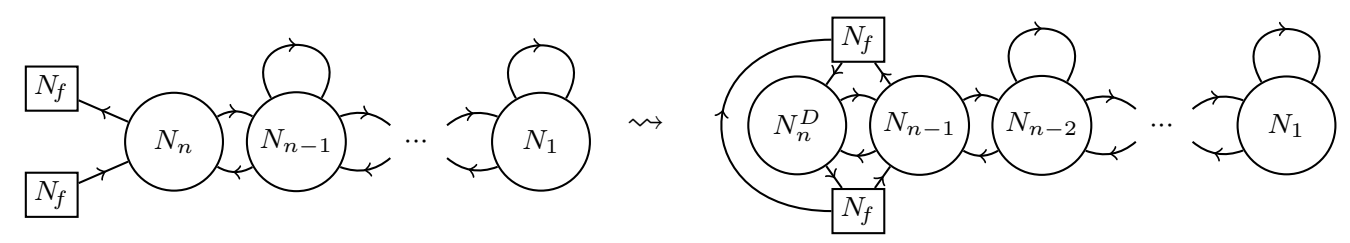

where the superpotential is the sum of all gauge (and flavour) invariant cubic combinations of bifundamental and adjoint chiral multiplets. The complexified FI parameters of the magnetic theory are $\hat{z}_{n}^{D}=\hat{z}_{n}^{-1}, \hat{z}_{n-1}^{D}=\hat{z}_{n} \hat{z}_{n-1}$, and $\hat{z}_{k}^{D}=\hat{z}_{k}$ for $k \leq n-2$.

The absence of adjoint chiral multiplet of $\mathrm{U}\left(N_{n-1}\right)$ in the second theory lets us apply Seiberg duality (and charge conjugation) to this node of the second quiver. Once more, the resulting quiver contains additional matter, including an adjoint of $\mathrm{U}\left(N_{n-2}\right)$ which cancels the already present adjoint because of a quadratic superpotential. The procedure can thus be continued by acting on successive nodes from $\mathrm{U}\left(N_{n}\right)$ to $\mathrm{U}\left(N_{1}\right)$. The resulting quivers are given in figure 3 .

We note in particular that the last quiver, obtained after applying Seiberg duality to all the nodes, has the same form as the original quiver: only one gauge group factor features fundamental and antifundamental chiral multiplets. This quiver gauge theory, or 


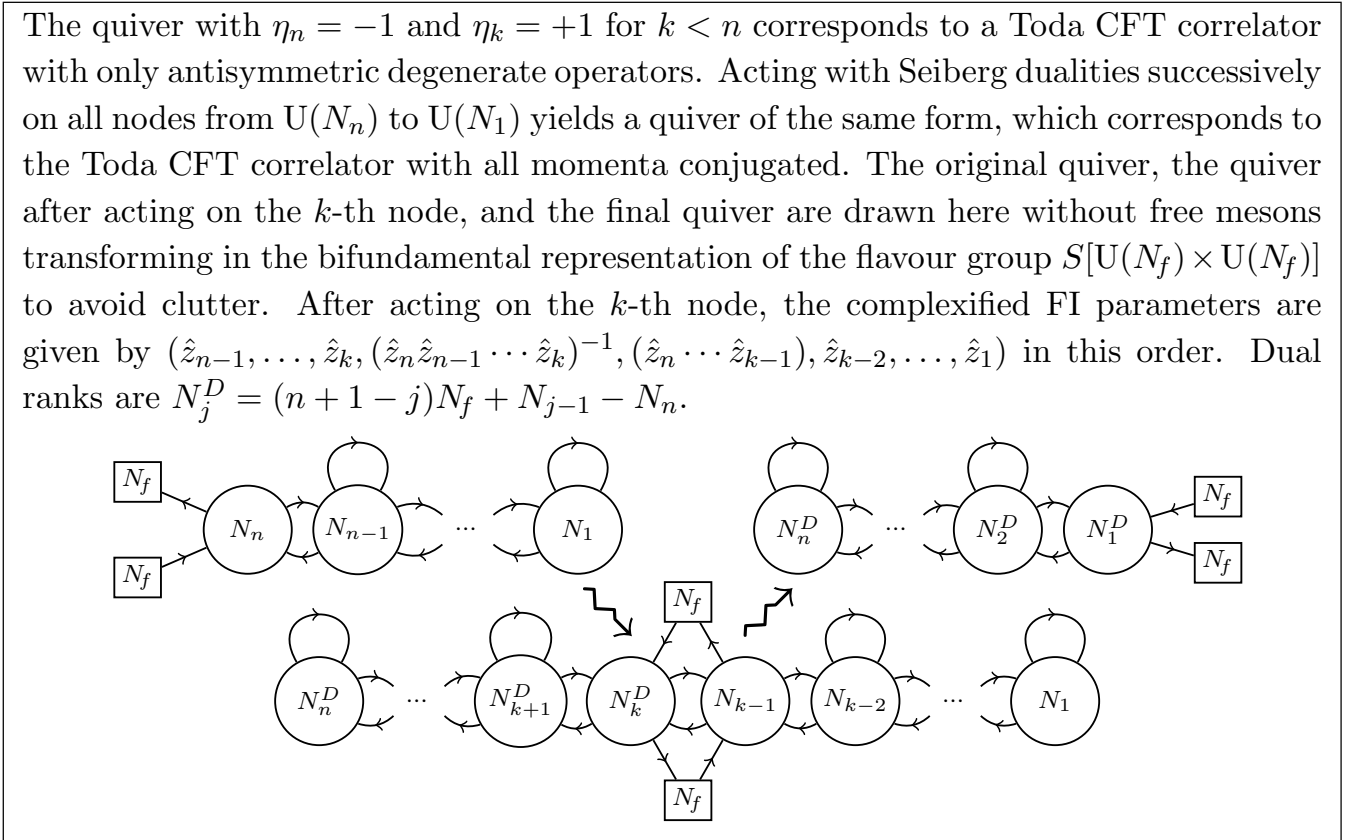

Figure 3. Sequence of Seiberg dualities on the quiver with all $\epsilon_{k}=-1$.

rather the $\mathcal{N}=(2,2)$ surface operator it defines in any class $\mathrm{S}$ theory, has a Toda CFT interpretation as the insertion of some degenerate vertex operators. Given the matter content of the gauge theory, all $n$ degenerate vertex operators are labeled by antisymmetric representations of $A_{N_{f}-1}$. The ranks of these representations are obtained from the number of colors in the dual theory:

$$
K_{j}^{D}=N_{j}^{D}-N_{j+1}^{D}=N_{f}-\left(N_{j}-N_{j-1}\right)=N_{f}-K_{j}
$$

for $1 \leq j \leq n$, where $N_{n+1}^{D}=N_{0}=0$. The positions of punctures are obtained from the FI parameters:

$$
x_{j}^{D}=\prod_{i=1}^{j} \hat{z}_{i}^{D}=\left[\prod_{i=j}^{n} \hat{z}_{i}\right]^{-1}=x_{j}^{-1} .
$$

Both of these maps are reproduced by conjugating all Toda CFT momenta and applying the conformal transformation $x \rightarrow x^{-1}$ to the correlator. This conformal transformation could be avoided by applying charge conjugation to all nodes of the quiver, mapping all complexified FI parameters to their inverse in the process.

All in all, Toda CFT conjugation translates to a combination of Seiberg dualities and charge conjugations. Here, the precise choice of matter content of the gauge theory is essential. On the gauge theory side, it ensures the absence of adjoint chiral multiplet at each step hence allows Seiberg duality to be applied. On the Toda CFT side, the conjugate of a symmetric representation is neither symmetric nor antisymmetric, thus momentum conjugation only yields symmetric or antisymmetric representations if the original representations were all antisymmetric. It should be noted that this choice of signs is identical to that made in section 2.4.2 to fuse degenerate punctures into a degenerate puncture labeled 
by an arbitrary representation, hence conjugating this representation corresponds to a set of Seiberg dualities on the gauge theory quiver.

We now go back to a quiver given by arbitrary signs $\eta_{k}$, and determine all dual descriptions obtained through Seiberg and $\mathcal{N}=(2,2)^{*}$ dualities. Inspired by the quivers which appeared when all $\epsilon_{k}=-1$, we consider the more general class of quivers

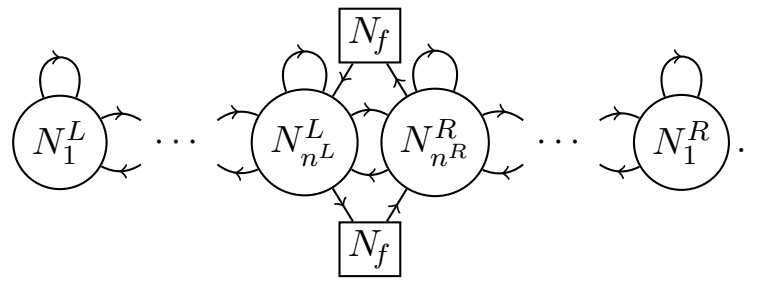

The multiplets described by this quiver are subject to a superpotential which depends on some signs $\eta_{k}^{L}$ for $1 \leq k \leq n^{L}$ and $\eta_{k}^{R}$ for $1 \leq k \leq n^{R}$. Namely, the superpotential is a sum of $W_{\eta^{L}}$ defined as in (2.117) for fields charged under the $\mathrm{U}\left(N_{k}^{L}\right), W_{\eta^{R}}$ for fields charged under the $\mathrm{U}\left(N_{k}^{R}\right)$, and two cubic terms coupling each bifundamental of $\mathrm{U}\left(N_{n^{L}}^{L}\right) \times \mathrm{U}\left(N_{n^{R}}^{R}\right)$ to multiplets charged under the flavour groups. For $n^{L}=0$ or $n^{R}=0$ we retrieve the quivers studied throughout this paper. Whenever $\eta_{k}^{L}=-1$, the superpotential contains a quadratic term $\operatorname{Tr}\left(\left(X_{k}^{L}\right)^{2}\right)$ which lets us integrate out the adjoint chiral multiplet $X_{k}^{L}$ of $\mathrm{U}\left(N_{k}^{L}\right)$, and similarly $\eta_{k}^{R}=-1$ lets us integrate out $X_{k}^{R}$.

Even though we have not given a Toda CFT interpretation for this class of quivers, we find analogues of the Toda CFT parameters $\left(x_{k}, \epsilon_{k}, K_{k}\right)$ which are simply transposed under dualities. Let $x_{n^{L}+1}^{L}=x_{n^{R}+1}^{R}=\epsilon_{n^{L}+1}^{L}=\epsilon_{n^{R}+1}^{R}=1$ and

$$
\begin{aligned}
& x_{j}^{L}=\prod_{i=j}^{n^{L}} \hat{z}_{i}^{L}, \quad \epsilon_{j}^{L}=\prod_{i=j}^{n^{L}} \eta_{i}^{L}, \quad K_{j}^{L}=N_{j}^{L}-N_{j-1}^{L} \quad \text { for } 1 \leq j \leq n^{L}, \\
& x_{j}^{R}=\prod_{i=j}^{n^{R}} \hat{z}_{i}^{R}, \quad \epsilon_{j}^{R}=\prod_{i=j}^{n^{R}} \eta_{i}^{R}, \quad K_{j}^{R}=N_{j}^{R}-N_{j-1}^{R} \quad \text { for } 1 \leq j \leq n^{R},
\end{aligned}
$$

where $N_{0}^{L}=N_{0}^{R}=0$.

Acting with Seiberg or the $\mathcal{N}=(2,2)^{*}$ duality (depending on $\eta_{k}^{L}$ ) on a node $\mathrm{U}\left(N_{k}^{L}\right)$ with $k<n^{L}$ exchanges $\left(x_{k}^{L}, \epsilon_{k}^{L}, K_{k}^{L}\right) \leftrightarrow\left(x_{k+1}^{L}, \epsilon_{k+1}^{L}, K_{k+1}^{L}\right)$. This is proven through the same calculations as for the case $n^{R}=0$ treated in section 3.3.1. Similarly, acting with a duality on $\mathrm{U}\left(N_{k}^{R}\right)$ with $k<n^{R}$ exchanges $\left(x_{k}^{R}, \epsilon_{k}^{R}, K_{k}^{R}\right) \leftrightarrow\left(x_{k+1}^{R}, \epsilon_{k+1}^{R}, K_{k+1}^{R}\right)$.

Let us now understand how dualities act on $\mathrm{U}\left(N_{n^{R}}^{R}\right)$. If $\epsilon_{n^{R}}^{R}=\eta_{n^{R}}^{R}=+1$, the fields which couple to the gauge group factor $\mathrm{U}\left(N_{n^{R}}^{R}\right)$ are those of $\mathcal{N}=(2,2)$ SQCDA, no simplification occurs, and neither Seiberg nor the $\mathcal{N}=(2,2)^{*}$ duality applies. However, if $\epsilon_{n^{R}}^{R}=\eta_{n^{R}}^{R}=-1$, we can integrate out the adjoint chiral multiplet to obtain SQCD with $N_{n^{R}}^{R}$ colors and $N_{f}+N_{n^{L}}^{L}+N_{n^{R}-1}^{R}$ flavours, and Seiberg duality yields a theory with $\left(N_{n^{R}}^{R}\right)^{D}=N_{f}+N_{n^{L}}^{L}+N_{n^{R}-1}^{R}-N_{n^{R}}^{R}$ colors. The magnetic theory has the same form (3.63) as the electric theory, but it features fundamental and antifundamental chiral multiplets of $U\left(\left(N_{n^{R}}^{R}\right)^{D}\right)$ and $\mathrm{U}\left(N_{n^{R}-1}^{R}\right)$ rather than $\mathrm{U}\left(N_{n^{L}}^{L}\right)$ and $\mathrm{U}\left(N_{n^{R}}^{R}\right)$ : in other words, $n^{L} \rightarrow n^{L}+1$ and $n^{R} \rightarrow n^{R}-1$. Due to the additional mesons after Seiberg duality, both $\eta_{n^{L}}^{L}$ and $\eta_{n^{R}-1}^{R}$ 
change signs, thus toggling between the presence or absence of an adjoint chiral multiplet. From our previous work on the action of Seiberg duality on quivers, we also know that FI parameters map as $\hat{z}_{n^{L}}^{L} \rightarrow \hat{z}_{n^{L}}^{L} \hat{z}_{n^{R}}^{R}, \hat{z}_{n^{R}}^{R} \rightarrow\left(\hat{z}_{n^{R}}^{R}\right)^{-1}$ and $\hat{z}_{n^{R}-1}^{R} \rightarrow \hat{z}_{n^{R}-1}^{R} \hat{z}_{n^{R}}^{R}$. Translating to the parameters $(x, \epsilon, K)$, we find that the set

$$
\left\{\left(\left(x_{j}^{L}\right)^{-1}, \epsilon_{j}^{L}, N_{f}-K_{j}^{L}\right) \mid 1 \leq j \leq n^{L}\right\} \cup\left\{\left(x_{j}^{R}, \epsilon_{j}^{R}, K_{j}^{R}\right) \mid 1 \leq j \leq n^{R}\right\}
$$

is unchanged: the triplet $\left(x_{n^{R}}^{R}, \epsilon_{n^{R}}^{R}, K_{n^{R}}^{R}\right)$ is simply moved from the second part of the set (on the right of flavour nodes) to the first part (on the left). By symmetry, the discussion applies to the node $\mathrm{U}\left(N_{n^{L}}^{L}\right)$ : if $\eta_{n^{L}}^{L}=+1$ there is no duality, while if $\eta_{n^{L}}^{L}=-1$ Seiberg duality moves $\left(\left(x_{n^{L}}^{L}\right)^{-1}, \epsilon_{n^{L}}^{L}, N_{f}-K_{n^{L}}^{L}\right)$ from the left part to the right part of (3.66).

All in all, Seiberg and $\mathcal{N}=(2,2)^{*}$ dualities acting on any of the nodes of (3.63) correspond to transpositions of $\left(\left(x_{1}^{L}\right)^{-1}, \epsilon_{1}^{L}, N_{f}-K_{1}^{L}\right), \ldots,\left(\left(x_{n^{L}}^{L}\right)^{-1}, \epsilon_{n^{L}}^{L}, N_{f}-K_{n^{L}}^{L}\right), \diamond$, $\left(x_{n^{R}}^{R}, \epsilon_{n^{R}}^{R}, K_{n^{R}}^{R}\right), \ldots,\left(x_{1}^{R}, \epsilon_{1}^{R}, K_{1}^{R}\right)$. The position of $\diamond$ indicates the position of the flavour nodes in the quiver. Only triplets $(x, \epsilon, K)$ with $\epsilon=-1$ can be exchanged with $\diamond$. Therefore, combinations of dualities correspond to all permutations which leave triplets with $\epsilon_{j}^{L}=+1$ to the left of $\diamond$ and those with $\epsilon_{j}^{R}=+1$ to the right of $\diamond$. Denoting by $n_{+}^{L}$ and $n_{+}^{R}$ the number of such triplets, and by $n_{-}$the total number of triplets with $\epsilon=-1$, we conclude that the number of dual descriptions of the theory (3.63) is

$$
\sum_{k=0}^{n_{-}}\left(\begin{array}{c}
n_{-} \\
k
\end{array}\right)\left(n_{+}^{L}+k\right) !\left(n_{+}^{R}+n_{-}-k\right) !=\frac{n_{+}^{L} ! n_{+}^{R !}\left(n_{+}^{L}+n_{+}^{R}+n_{-}+1\right) !}{\left(n_{+}^{L}+n_{+}^{R}+1\right) !} .
$$

As a last comment, we propose that the partition function of the $S^{2}$ surface operator defined by coupling (3.63) to $N_{f}^{2}$ free hypermultiplets on $S_{b}^{4}$ should be equal to

$$
Z_{S^{2} \subset S_{b}^{4}}^{(3.63)}=\left\langle\widehat{V}_{\alpha_{\infty}}(\infty) \widehat{V}_{\hat{m}}(1) \widehat{V}_{\alpha_{0}}(0) \prod_{j=1}^{n^{L}} \widehat{V}_{-b \Omega\left(K_{j}^{L}, \epsilon_{j}^{L}\right)^{C}}\left(\left(x_{j}^{L}\right)^{-1}\right) \prod_{j=1}^{n^{R}} \widehat{V}_{-b \Omega\left(K_{j}^{R}, \epsilon_{j}^{R}\right)}\left(x_{j}^{R}\right)\right\rangle
$$

up to factors that can be absorbed in $Z$. Here, $\Omega(K,+1)=K h_{1}$ is the highest weight of a symmetric representation while $\Omega(K,-1)=\omega_{K}$ is the highest weight of an antisymmetric representation. The proposal is consistent with the action of dualities as permutations of $(x, \epsilon, K)$ triplets described above: in particular, an antisymmetric representation with highest weight $\omega_{K}$ can be seen either as part of the left product $\left(\omega_{K}=\Omega\left(N_{f}-K,-1\right)^{C}\right)$ or as part of the right product $\left(\omega_{K}=\Omega(K,-1)\right)$, and this choice reproduces the Seiberg duality map. On the contrary, the conjugate of a symmetric representation is neither symmetric nor antisymmetric, so punctures with $\epsilon=+1$ belong to a given product and cannot be moved to the other one. We have not explored this proposal further, as fusions of antisymmetric representations are enough to obtain arbitrary representations.

\section{Acknowledgments}

We would like to thank Davide Gaiotto, Peter Koroteev, Daniel Park, and Jörg Teschner for useful discussions. B.L.F. would like to thank the Perimeter Institute for hospitality. 
Research at Perimeter Institute is supported by the Government of Canada through Industry Canada and by the Province of Ontario through the Ministry of Economic Development and Innovation. J.G. also acknowledges further support from an NSERC Discovery Grant and from an ERA grant by the Province of Ontario.

\section{A Toda CFT}

This appendix is devoted to the $A_{N_{f}-1}$ Toda CFT, a generalization of the Liouville CFT $\left(N_{f}=2\right)$, and can be read independently. It is split into five topics: we review notations and basic properties (appendix A.1), match products of three-point functions with gauge theory one-loop determinants (appendix A.2), derive new braiding matrices useful in the main text (appendix A.3), list known fusion rules and find new ones (appendix A.4), deduce new conformal blocks from the correspondence (appendix A.5) and finally define some irregular punctures (appendix A.6).

\section{A.1 Basic properties}

We describe here some properties of the $A_{N_{f}-1}$ Toda CFT, omitting some details which can be found in [58]. We introduce the normalizations (A.7) and (A.8) of vertex operators, which simplify three-point functions hence simplify constant factors in the main text.

Microscopically, the theory describes a scalar field $\varphi$ in the Cartan subalgebra of $A_{N_{f}-1}$, minimally coupled to the metric, with an exponential potential term. It depends on a coupling constant $b$, and a cosmological constant $\mu$. We will use the combination

$$
\hat{\mu}=\left[\pi \mu \gamma\left(b^{2}\right) b^{2-2 b^{2}}\right]^{1 / b}
$$

where $\gamma(x)=\Gamma(x) / \Gamma(1-x)$, because the theory is (non-manifestly) invariant under $(b, \hat{\mu}) \rightarrow$ $(1 / b, \hat{\mu})$. Besides its local symmetry algebra $W_{N_{f}}$ (a higher-spin extension of the Virasoro algebra $W_{2}$ ), the theory also possesses a discrete symmetry $\varphi \rightarrow \varphi^{C}$, defined as the $\mathbb{C}$-linear map such that

$$
h_{s}^{C}=-h_{N_{f}+1-s}
$$

for all $1 \leq s \leq N_{f}$, where $h_{s}$ are the weights of the fundamental representation of $A_{N_{f}-1}$. These weights form an overcomplete basis $\left(h_{1}+\cdots+h_{N_{f}}=0\right)$ of linear forms over the Cartan subalgebra of $A_{N_{f}-1}$. In principle, one should distinguish the space of linear forms from the Cartan subalgebra, but the (bilinear) Killing form $\langle$,$\rangle defined by$

$$
\left\langle h_{s}, h_{t}\right\rangle=\delta_{s t}-\frac{1}{N_{f}}
$$

identifies the two spaces. Note that the Killing form is invariant under conjugation, and that $\left(\varphi^{C}\right)^{C}=\varphi$. Conjugation maps the highest weight of a representation to the highest weight of the conjugate representation, hence its name.

Vertex operators $V_{\alpha}=e^{\langle\alpha, \varphi\rangle}$, labeled by their momentum $\alpha$ in the Cartan subalgebra, are primary operators for the $W_{N_{f}}$ symmetry algebra. The symmetry $\varphi \rightarrow \varphi^{C}$ maps $V_{\alpha}=$ $e^{\langle\alpha, \varphi\rangle}$ to $e^{\left\langle\alpha, \varphi^{C}\right\rangle}=e^{\left\langle\alpha^{C}, \varphi\right\rangle}=V_{\alpha C}$, and since simple roots are permuted under conjugation, correlators of vertex operators are invariant under conjugating all momenta $\alpha_{i} \rightarrow \alpha_{i}^{C}$. 
Each vertex operator $V_{\alpha}$ is additionally invariant up to a constant factor (reflection amplitude) under the Weyl group of $A_{N_{f}-1}$, which acts by permuting the $N_{f}$ components $\left\langle\alpha-Q, h_{s}\right\rangle$. Here, $Q=\left(b+\frac{1}{b}\right) \rho$ is a multiple of the sum

$$
\rho=\frac{1}{2} \sum_{e>0} e=\frac{1}{2} \sum_{s<t}^{N_{f}}\left(h_{s}-h_{t}\right)=\sum_{s} \frac{N_{f}+1-2 s}{2} h_{s}
$$

of all positive roots $e=h_{s}-h_{t}, 1 \leq s<t \leq N_{f}$, of $A_{N_{f}-1}$. The invariance of $V_{\alpha}$ is confirmed by noting that its dimension

$$
\Delta(\alpha)=\frac{1}{2}\langle\alpha, 2 Q-\alpha\rangle,
$$

and quantum numbers associated to higher spin generators of $W_{N_{f}}$, are invariant under Weyl reflections. The normalization (A.7) later on absorbs reflection amplitudes.

When decomposing $n$-point functions into products of three-point functions and conformal blocks, we must take into account two-point functions as well. Non-zero two-point functions are $\left\langle V_{\alpha} V_{2 Q-\alpha}\right\rangle$ and Weyl reflections thereof. As a result, the momenta which appear in two neighboring three-point functions of the decomposition are related by the map $\alpha \rightarrow 2 Q-\alpha$. For the Liouville CFT $\left(N_{f}=2\right)$, those momenta are Weyl reflections of each other, hence the distinction is irrelevant. For the general $A_{N_{f}-1}$ Toda CFT, one must include an orientation when labeling conformal blocks by the various internal momenta, and reversing the orientation amounts to changing $\alpha \rightarrow 2 Q-\alpha$. External momenta can also be given an orientation (which must then be retained for correlators as well as conformal blocks), where an "incoming" momentum $\alpha$ denotes the presence of the vertex operator $V_{\alpha}$, and an "outgoing" momentum $\alpha$ denotes $V_{2 Q-\alpha}$. This incoming/outgoing distinction also affects the relation between fusion rules and non-zero three-point functions.

Generically, vertex operators generate irreducible representations of $W_{N_{f}}$. Semidegenerate vertex operators are defined by the presence of null-vectors among their $W_{N_{f}}$ descendants. In this paper, all semi-degenerate vertex operators take the form $V_{\varkappa h_{1}}$ (and $V_{-\lambda h_{N_{f}}}$ ). The conjugate momentum $\left(\varkappa h_{1}\right)^{C}=-\varkappa h_{N_{f}}$ is in fact mapped by the Weyl reflection defined by the permutation $\left(12 \cdots N_{f}\right)$ to the original form

$$
\varkappa^{D} h_{1}=\left[N_{f}\left(b+\frac{1}{b}\right)-\varkappa\right] h_{1}
$$

since $\left\langle\varkappa h_{1}^{C}-Q, h_{N_{f}}\right\rangle=\left\langle\varkappa^{D} h_{1}-Q, h_{1}\right\rangle$, and $\left\langle\varkappa h_{1}^{C}-Q, h_{s-1}\right\rangle=\left\langle\varkappa^{D} h_{1}-Q, h_{s}\right\rangle$ for all $2 \leq s \leq N_{f}$. Thus, $V_{\varkappa h_{1}^{C}}$ and $V_{\varkappa^{D} h_{1}}$ are equal up to a reflection amplitude, absorbed by the normalization (A.8). This equality is crucial to obtain dualities in section 3.1 and section 3.2.2 as conjugation of momenta.

Fully degenerate momenta $\alpha=-b \omega-\frac{1}{b} \omega^{\prime}$ are labeled by pairs $\left(\omega, \omega^{\prime}\right)$ of highest weights of $A_{N_{f}-1}$ representations. We only consider in this work degenerate momenta of the form $\alpha=-b \omega$, and mapping $b \rightarrow \frac{1}{b}$ would probe degenerate momenta $\alpha=-\frac{1}{b} \omega$, but the mixed case with non-zero $\omega$ and $\omega^{\prime}$ is hard to access. We denote the representation of $A_{N_{f}-1}$ with highest weight $\omega$ by $\mathcal{R}(\omega)$. In particular, the fundamental representation 
$\mathcal{R}\left(h_{1}\right)$ has weights $h_{s}$ for $1 \leq s \leq N_{f}$, and highest weight $h_{1}$. The $N$-th antisymmetric power of $\mathcal{R}\left(h_{1}\right)$ is $\mathcal{R}\left(\omega_{N}\right)$, where $\omega_{N}=\sum_{j=1}^{N} h_{j}$; it has weights $h_{\{p\}}=\sum_{j=1}^{N} h_{p_{j}}$ for $1 \leq p_{1}<\cdots<p_{N} \leq N_{f}$. The $N$-th symmetric power $\mathcal{R}\left(N h_{1}\right)$ has weights $\sum_{j=1}^{N} h_{p_{j}}$ for $1 \leq p_{1} \leq \cdots \leq p_{N} \leq N_{f}$, or equivalently $h_{[n]}=\sum_{s=1}^{N_{f}} n_{s} h_{s}$ for (non-negative) integers $n_{1}+\cdots+n_{N_{f}}=N$. We also consider quasi-rectangular Young diagrams: for $0 \leq j<l$ and $0 \leq k<N_{f}$, the highest weight $\omega_{k l+j, l}=l \omega_{k}+j h_{k+1}$ corresponds to a Young diagram with $k l+j$ boxes, organized as $k$ rows of $l$ boxes, followed by a $j$-box row. This reproduces the antisymmetric case $\omega_{N, 1}=\omega_{N}$ for $l=1$, and the symmetric case $\omega_{N, l}=N h_{1}$ for $l \geq N$.

In view of the matching of parameters with gauge theory, we write generic momenta as $\alpha=Q-\mathrm{i} a$. The dimension is $\Delta(Q-\mathrm{i} a)=\frac{1}{2}\langle Q, Q\rangle-\frac{1}{2}\langle\mathrm{i} a, \mathrm{i} a\rangle$. Weyl reflections act by permuting the $\left\langle a, h_{s}\right\rangle$. In terms of the Upsilon function (A.9) below, we introduce the normalization

$$
\widehat{V}_{Q-\mathrm{i} a}=\frac{\hat{\mu}^{-\langle\mathrm{i} a, \rho\rangle}}{\prod_{s<t}^{N_{f}} \Upsilon\left(\left\langle\mathrm{i} a, h_{s}-h_{t}\right\rangle\right)} V_{Q-\mathrm{i} a}
$$

where the product ranges over positive roots $e=h_{s}-h_{t}$. The normalization factor is invariant under conjugation, hence does not spoil this symmetry of Toda CFT correlators involving generic operators $\widehat{V}_{\alpha}$. The three-point function $\left\langle\widehat{V}_{\alpha} \widehat{V}_{\alpha^{\prime}} \widehat{V}_{\varkappa h_{1}}\right\rangle$ given in (A.13) is invariant under Weyl reflections permuting the $\left\langle a, h_{s}\right\rangle$, hence the normalized operator $\widehat{V}_{\alpha}$ is Weyl invariant. To further simplify three-point functions, we also provide a normalization for semi-degenerate operators and fully degenerate operators,

$$
\widehat{V}_{\varkappa h_{1}}=\frac{\hat{\mu}^{\left\langle\varkappa h_{1}, \rho\right\rangle}}{(\Upsilon(b))^{N_{f}-1} \Upsilon(\varkappa)} V_{\varkappa h_{1}}, \quad \widehat{V}_{-b \omega}=\left[\hat{\mu} b^{2\left(b+\frac{1}{b}\right)}\right]^{\langle-b \omega, \rho\rangle} V_{-b \omega} .
$$

The normalizations of generic and semi-degenerate operators are invariant under $b \rightarrow \frac{1}{b}$.

The Upsilon function appearing above depends implicitly on the coupling constant $b$ (it is invariant under $b \rightarrow \frac{1}{b}$ ), and for generic real $b$ it is a holomorphic function, uniquely determined by its normalization $\Upsilon\left(\frac{1}{2}\left(b+\frac{1}{b}\right)\right)=1$ and by shift relations

$$
\Upsilon(x+b)=\gamma(b x) b^{1-2 b x} \Upsilon(x), \quad \Upsilon(x+1 / b)=\gamma(x / b) b^{2 x / b-1} \Upsilon(x) .
$$

Also, $\Upsilon\left(b+\frac{1}{b}-x\right)=\Upsilon(x)$ and the function has zeros at $-m b-n \frac{1}{b}$ and $(m+1) b+(n+1) \frac{1}{b}$ for integers $m, n \geq 0$, and no poles. As $x \rightarrow \pm \mathrm{i} \infty$, one has

$$
\begin{aligned}
& \frac{\Upsilon(x+a)}{\Upsilon(x)} \sim\left(\frac{-x^{2}}{e^{2}}\right)^{a x}|x|^{a(a-b-1 / b)} \sim\left(\gamma(b x) b^{1-2 b x}\right)^{a / b}|x|^{a(a-b)}, \\
& \prod_{s=1}^{N_{f}} \frac{\Upsilon\left(x+\left\langle\alpha, h_{s}\right\rangle\right)}{\Upsilon(x)} \sim|x|^{\langle\alpha, \alpha\rangle},
\end{aligned}
$$

for any $a$ and any momentum $\alpha$. The gamma function $\gamma(x)=\Gamma(x) / \Gamma(1-x)$ obeys by construction $\gamma(1-x)=1 / \gamma(x)$ and also appears in one-loop determinants of chiral multiplets. Vortex partition functions involve Pochhammer symbols

$$
(x)_{k}=\frac{\Gamma(x+k)}{\Gamma(x)}=(-1)^{k} \frac{\Gamma(1-x)}{\Gamma(1-x-k)}=\frac{(-1)^{k}}{(1-x)_{-k}} .
$$

This equality is shown using the Euler identity $\Gamma(x) \Gamma(1-x)=\pi / \sin \pi x$. 


\section{A.2 Three-point functions}

We check in this appendix that the one-loop determinants which appear in Higgs branch expansions of $S^{2}$ partition functions, considered in the main text, are equal to products of three-point functions which appear in s- and u-channel decompositions of the corresponding Toda CFT correlators. This relies on the three-point functions provided by [58], equations (1.39), (1.51), and (1.56), which we first translate to our normalization.

The three-point function of two generic operators $\widehat{V}_{Q-\mathrm{i} a_{1}}$ and $\widehat{V}_{Q-\mathrm{i} a_{2}}$ and of a semidegenerate operator $\widehat{V}_{\varkappa h_{1}}$ is expressed as the normalizations (A.7) and (A.8) multiplied by equation (1.39) of [58] with all momenta conjugated:

$$
\begin{aligned}
\widehat{C}\left(Q-\mathrm{i} a_{1}, Q-\mathrm{i} a_{2}, \varkappa h_{1}\right) & =\frac{\hat{\mu}^{-\left\langle\mathrm{i} a_{1}+\mathrm{i} a_{2}-\varkappa h_{1}, \rho\right\rangle} C\left(Q-\mathrm{i} a_{1}^{C}, Q-\mathrm{i} a_{2}^{C}, \varkappa h_{1}^{C}\right)}{(\Upsilon(b))^{N_{f}-1} \Upsilon(\varkappa) \prod_{s<t}^{N_{f}} \Upsilon\left(\left\langle\mathrm{i} a_{1}, h_{s}-h_{t}\right\rangle\right) \Upsilon\left(\left\langle\mathrm{i} a_{2}, h_{s}-h_{t}\right\rangle\right)} \\
& =\frac{1}{\prod_{s, t=1}^{N_{f}} \Upsilon\left(\frac{\varkappa}{N_{f}}+\left\langle\mathrm{i} a_{1}, h_{s}\right\rangle+\left\langle\mathrm{i} a_{2}, h_{t}\right\rangle\right)} .
\end{aligned}
$$

The three-point function is invariant under Weyl transformations of each $\widehat{V}_{Q-\mathrm{i} a_{i}}$, which permute the $\left\langle\mathrm{i} a_{i}, h_{s}\right\rangle$, hence the normalized $\widehat{V}_{Q-\mathrm{i} a}$ are Weyl invariant, as claimed earlier. The three-point function is also invariant under conjugation of all momenta, followed by the Weyl transformation (A.6) which maps $\left(\varkappa h_{1}\right)^{C} \rightarrow \varkappa^{D} h_{1}=\left(N_{f}(b+1 / b)-\varkappa\right) h_{1}$ : indeed, $\left\langle\mathrm{i} a_{i}, h_{s}\right\rangle \rightarrow-\left\langle\mathrm{i} a_{i}, h_{s}\right\rangle$ and $\varkappa / N_{f} \rightarrow(b+1 / b)-\varkappa / N_{f}$ under this transformation, and we know that $\Upsilon(b+1 / b-x)=\Upsilon(x)$.

Besides this three-point function, we also need some three-point functions involving a degenerate operator $\widehat{V}_{-b \omega}$. The OPE of this operator with a generic $\widehat{V}_{Q-\mathrm{i} a}$ is

$$
\widehat{V}_{-b \omega} \widehat{V}_{Q-\mathrm{i} a}=\sum_{h \in \mathcal{R}(\omega)} \widehat{C}_{-b \omega, Q-\mathrm{i} a}^{Q-\mathrm{i} a-b h}\left[\widehat{V}_{Q-\mathrm{i} a-b h}\right],
$$

where the sum runs over weights of $\mathcal{R}(\omega)$ and the brackets denote the contribution from $W_{N_{f}}$ descendants (see appendix A.4 for a description of which momenta can appear in various OPE). The structure constants $\widehat{C}_{-b \omega, Q-\mathrm{i} a}^{Q-\mathrm{i} a-b h}$ are equal to their analogues given in [58] for usual vertex operators, multiplied by the normalization factors of $\widehat{V}_{-b \omega}$ and $\widehat{V}_{Q-\mathrm{i} a}$, and divided by the normalization of $\widehat{V}_{Q-i a-b h}$, namely

$$
\widehat{C}_{-b \omega, Q-\mathrm{i} a}^{Q-\mathrm{i} a-b h}=\hat{\mu}^{\langle b h-b \omega, \rho\rangle} b^{2\langle-b \omega, Q\rangle} \prod_{s<t}^{N_{f}}\left[\frac{\Upsilon\left(\left\langle\mathrm{i} a+b h, h_{s}-h_{t}\right\rangle\right)}{\Upsilon\left(\left\langle\mathrm{i} a, h_{s}-h_{t}\right\rangle\right)}\right] C_{-b \omega, Q-\mathrm{i} a}^{Q-\mathrm{i} a-b h} .
$$

The structure constants are also closely related to three-point functions:

$$
\widehat{C}_{-b \omega, Q-\mathrm{i} a}^{Q-\mathrm{i} a-b h}=\prod_{s \neq t}^{N_{f}}\left[\Upsilon\left(\left\langle\mathrm{i} a+b h, h_{s}-h_{t}\right\rangle\right)\right] \widehat{C}(-b \omega, Q-\mathrm{i} a, Q+\mathrm{i} a+b h) .
$$

The change $Q-\mathrm{i} a-b h \rightarrow Q+\mathrm{i} a+b h$ and the Upsilon functions both come from the non-zero two-point functions $\left\langle\widehat{V}_{\alpha}(z, \bar{z}) \widehat{V}_{2 Q-\alpha}(0)\right\rangle=|z|^{-4 \Delta(\alpha)} / \prod_{s \neq t}^{N_{f}} \Upsilon\left(\left\langle Q-\alpha, h_{s}-h_{t}\right\rangle\right)$. 
Equation (1.51) of [58] covers the case of a degenerate field $\widehat{V}_{-b \omega_{N}}$ labeled by the antisymmetric representation $\mathcal{R}\left(\omega_{N}\right)$, whose weights $h=h_{p_{1}}+\cdots+h_{p_{N}}$ are labeled by $N$-element subsets of $\left\{1, \ldots, N_{f}\right\}$ without repetition. With our normalization (A.15), all Upsilon functions cancel through the shift relation (A.9), and leave only gamma functions:

$$
\widehat{C}_{-b \omega_{N}, Q-\mathrm{i} a}^{Q-\mathrm{i} a-b h}=b^{-N_{f}\langle 2 \mathrm{i} a+b h, b h\rangle} \prod_{s \notin\{p\}}^{N_{f}} \prod_{t \in\{p\}} \gamma\left(b\left\langle\mathrm{i} a, h_{t}-h_{s}\right\rangle\right) .
$$

When matching with the $S^{2}$ partition function of SQCDA, we need three-point functions involving $\widehat{V}_{-N b h_{1}}$. Weights of the $N$-th symmetric representation $\mathcal{R}\left(N h_{1}\right)$ are $h=\sum_{s=1}^{N_{f}} n_{s} h_{s}$ for a choice of $N_{f}$ integers $n_{s} \geq 0$ with $n_{1}+\cdots+n_{N_{f}}=N$. The three-point function can be derived from (A.13) by setting $\varkappa=-N b$, taking into account the normalization, and extracting the residue at $\mathrm{i} a_{1}=\mathrm{i} a$ and $\mathrm{i} a_{2}=-\mathrm{i} a-b h$. This yields

$$
\widehat{C}_{-N b h_{1}, Q-\mathrm{i} a}^{Q-\mathrm{i} a-b h}=\frac{b^{-N_{f}\langle 2 \mathrm{i} a+b h, b h\rangle}}{\prod_{\nu=1}^{N} \gamma\left(-\nu b^{2}\right)} \prod_{s, t=1}^{N_{f}} \prod_{\nu=0}^{n_{t}-1} \gamma\left(b\left\langle\mathrm{i} a, h_{t}-h_{s}\right\rangle+\left(\nu-n_{s}\right) b^{2}\right) .
$$

Taking $N=1$ in either (A.17) or (A.18) yields the (same) expression for the case of a fundamental degenerate field,

$$
\widehat{C}_{-b h_{1}, Q-\mathrm{i} a}^{Q-\mathrm{i} a-b h_{p}}=b^{-N_{f}\left\langle 2 \mathrm{i} a+b h_{p}, b h_{p}\right\rangle} \prod_{s \neq p}^{N_{f}} \gamma\left(b\left\langle\mathrm{i} a, h_{p}-h_{s}\right\rangle\right) .
$$

For theories with a superpotential, we also use some three-point functions with a degenerate $\widehat{V}_{-b e_{0}}$ labeled by the highest weight $e_{0}=h_{1}-h_{N_{f}}$ of the adjoint representation. Because the weight 0 has multiplicity in this representation, $\widehat{C}_{-b\left(h_{1}-h_{N_{f}}\right), \alpha}^{\alpha}$ is not a ratio of Gamma functions. We will focus on other weights $h=h_{i}-h_{j}$, which have no multiplicity. From equation (1.56) of [58],

$$
\widehat{C}_{-b e_{0}, Q-\mathrm{i} a}^{Q-i \mathrm{a} a h}=b^{-N_{f}\langle 2 \mathrm{i} a+b h, b h\rangle} \frac{\gamma\left(b\left\langle\mathrm{i} a, h_{i}-h_{j}\right\rangle+b^{2}\right)}{\gamma\left(b\left\langle\mathrm{i} a, h_{i}-h_{j}\right\rangle\right)} \prod_{s \neq i}^{N_{f}} \gamma\left(b\left\langle\mathrm{i} a, h_{i}-h_{s}\right\rangle\right) \prod_{s \neq j}^{N_{f}} \gamma\left(b\left\langle\mathrm{i} a, h_{s}-h_{j}\right\rangle\right) .
$$

We are now ready to consider the products of three-point functions appearing in sand u-channel decompositions of Toda CFT correlators of interest. Our first computation concerns the s-channel decomposition (2.44) of a four-point function with the degenerate insertion $\widehat{V}_{-b h_{1}}$, which corresponds to the $S^{2}$ partition function of SQED, multiplied by the contribution $Z_{S_{b}^{4}}^{\text {free }}=\widehat{C}\left(\alpha_{\infty}, \alpha_{0}, \varkappa h_{1}\right)$ of free hypermultiplets. We set $\alpha_{\infty}=Q-\mathrm{i} a_{\infty}$ and $\alpha_{0}=Q-\mathrm{i} a_{0}$, and evaluate:

$$
\begin{aligned}
\widehat{C}_{-b h_{1}, \alpha_{0}}^{\alpha_{0}-b h_{p}} \widehat{C}\left(\alpha_{\infty},\right. & \left.\alpha_{0}-b h_{p},(\varkappa+b) h_{1}\right) / \widehat{C}\left(\alpha_{\infty}, \alpha_{0}, \varkappa h_{1}\right) \\
& =\widehat{C}_{-b h_{1}, \alpha_{0}}^{\alpha_{0}-b h_{p}} \prod_{s, t=1}^{N_{f}}\left[\frac{\Upsilon\left(\frac{\varkappa}{N_{f}}+\left\langle\mathrm{i} a_{0}, h_{s}\right\rangle+\left\langle\mathrm{i} a_{\infty}, h_{t}\right\rangle\right)}{\Upsilon\left(\frac{\varkappa}{N_{f}}+\left\langle\mathrm{i} a_{0}, h_{s}\right\rangle+\left\langle\mathrm{i} a_{\infty}, h_{t}\right\rangle+b \delta_{p s}\right)}\right] \\
& =\underbrace{b^{2 b \varkappa-N_{f}\left(1+b^{2}\right)+b^{2}}}_{A^{-1}} \frac{\prod_{s \neq p}^{N_{f}} \gamma\left(b\left\langle\mathrm{i} a_{0}, h_{p}-h_{s}\right\rangle\right)}{\prod_{t=1}^{N_{f}} \gamma\left(\frac{b \varkappa}{N_{f}}+b\left\langle\mathrm{i} a_{0}, h_{p}\right\rangle+b\left\langle\mathrm{i} a_{\infty}, h_{t}\right\rangle\right)} .
\end{aligned}
$$


The numerator gamma function is $\gamma\left(\mathrm{i} m_{p}-\mathrm{i} m_{s}\right)$ in terms of gauge theory twisted masses, and the denominator is $\gamma\left(1+\mathrm{i} m_{p}+\mathrm{i} \widetilde{m}_{t}\right)$. We thus recognize the one-loop determinant appearing in the s-channel decomposition (2.32) of $Z$, divided by the constant $A$ given in (2.23). Since $A$ is invariant under the exchange of $\alpha_{0}$ and $\alpha_{\infty}$, which amounts to exchanging the s- and $\mathrm{u}$-channels, the matching of three-point functions and one-loop contributions also occurs in the u-channel.

Next is the case of SQCD. The corresponding Toda four-point function involves the degenerate insertion $\widehat{V}_{-b \omega_{N}}$, the semi-degenerate $\widehat{V}_{(\varkappa+N b) h_{1}}$, and two generic momenta $\alpha_{0}=$ $Q-\mathrm{i} a_{0}$ and $\alpha_{\infty}=Q-\mathrm{i} a_{\infty}$. We factor out the $S_{b}^{4}$ contribution $\widehat{C}\left(\alpha_{\infty}, \alpha_{0}, \varkappa h_{1}\right)$. For a given weight $h=h_{p_{1}}+\cdots+h_{p_{N}}$ of $\mathcal{R}\left(\omega_{N}\right)$, we find

$$
\begin{aligned}
\widehat{C}_{-b \omega_{N}, \alpha_{0}}^{\alpha_{0}-b h} \widehat{C}\left(\alpha_{\infty}, \alpha_{0}-b h,(\varkappa+N b) h_{1}\right) / \widehat{C}\left(\alpha_{\infty}, \alpha_{0}, \varkappa h_{1}\right) \\
=\widehat{C}_{-b \omega_{N}, \alpha_{0}}^{\alpha_{0}-b h} \prod_{s, t=1}^{N_{f}}\left[\frac{\Upsilon\left(\frac{\varkappa}{N_{f}}+\left\langle\mathrm{i} a_{0}, h_{t}\right\rangle+\left\langle\mathrm{i} a_{\infty}, h_{s}\right\rangle\right)}{\Upsilon\left(\frac{\varkappa}{N_{f}}+\left\langle\mathrm{i} a_{0}, h_{t}\right\rangle+\left\langle\mathrm{i} a_{\infty}, h_{s}\right\rangle+b \delta_{t \in\{p\}}\right)}\right] \\
=\underbrace{b^{2 N b \varkappa-N N_{f}\left(1+b^{2}\right)+N^{2} b^{2}}}_{A^{-1}} \prod_{t \in\{p\}}\left[\frac{\prod_{s \notin\{p\}}^{N_{f}} \gamma\left(-b\left\langle\mathrm{i} a_{0}, h_{s}-h_{t}\right\rangle\right)}{\prod_{s=1}^{N_{f}} \gamma\left(\frac{b \varkappa}{N_{f}}+b\left\langle\mathrm{i} a_{0}, h_{t}\right\rangle+b\left\langle\mathrm{i} a_{\infty}, h_{s}\right\rangle\right)}\right] .
\end{aligned}
$$

Again, we recognize the ratio of $\gamma\left(-\mathrm{i} m_{s}+\mathrm{i} m_{t}\right)$ and $\gamma\left(1+\mathrm{i} \widetilde{m}_{s}+\mathrm{i} m_{t}\right)$ as being the one-loop determinants (2.58) of SQCD. This fixes the constant $A$ to be (2.55) in the matching with SQCD. Since $A$ is invariant under the exchange of $\alpha_{0}$ and $\alpha_{\infty}$, the u-channel three-point functions and one-loop determinant match up to the same constant.

Our last four-point function corresponds to the $S^{2}$ partition function of SQCDA, and involves the degenerate field $\widehat{V}_{-N b h_{1}}$. With notations as above, and for $h=\sum_{s} n_{s} h_{s}$, we compute

$$
\begin{aligned}
& \widehat{C}_{-N b h_{1}, \alpha_{0}}^{\alpha_{0}-b h} \widehat{C}\left(\alpha_{\infty}, \alpha_{0}-b h,(\varkappa+N b) h_{1}\right) / \widehat{C}\left(\alpha_{\infty}, \alpha_{0}, \varkappa h_{1}\right)
\end{aligned}
$$

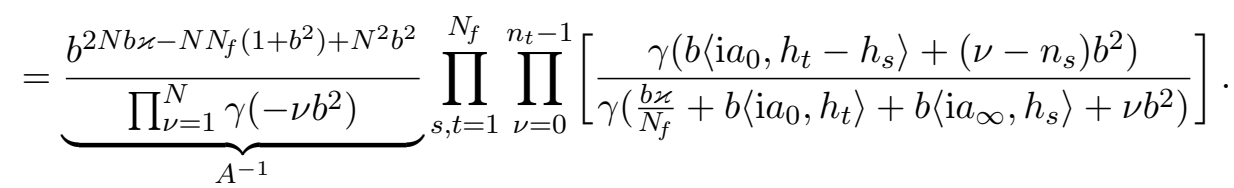

In terms of gauge theory variables, the numerator gamma functions have arguments $i_{t}+$ $\nu b^{2}-\mathrm{i} m_{s}-n_{s} b^{2}$, while the denominator have arguments $1+\mathrm{i} \widetilde{m}_{s}+\mathrm{i} m_{t}+\nu b^{2}$, hence we obtain the one-loop determinant (2.96), divided by the constant $A$ given in (2.92). Once more, $A$ is invariant under $\alpha_{0} \leftrightarrow \alpha_{\infty}$, hence u-channel three-point functions and one-loop determinants match.

Perhaps interestingly, the power of $b$ appearing in $A$ can be recast as

$$
b^{N N_{f}\left(1+b^{2}\right)-N^{2} b^{2}-2 N b \varkappa}=b^{-\varkappa\left[N_{f}\left(b+\frac{1}{b}\right)-\varkappa\right]} / b^{-(\varkappa+N b)\left[N_{f}\left(b+\frac{1}{b}\right)-\varkappa-N b\right]} .
$$

We do not absorb these powers of $b$ into the normalization of $\widehat{V}_{\varkappa h_{1}}$ and $\widehat{V}_{(\varkappa+N b) h_{1}}$, because they would spoil the $b \rightarrow \frac{1}{b}$ symmetry which (A.8) enjoys. Note that these powers are invariant under conjugation, which maps $\varkappa \rightarrow N_{f}\left(b+\frac{1}{b}\right)-\varkappa$ and $\varkappa+N b$ similarly. 


\section{A.3 Braiding matrices}

In this appendix, we compute the braiding matrix of the antisymmetric degenerate operator $V_{-b \omega_{N}}$ around the semi-degenerate operator $V_{\varkappa h_{1}}$, as well as its gauge theory analogue, and check that they are equal.

\section{A.3.1 Gauge theory transfer matrices}

Let us start on the gauge theory side: namely, we find the matrix relating Higgs branch decompositions near $z=0$ and near $z=\infty$ of the partition function (2.58) of SQCD.

First, focus on the case of SQED $(N=1)$, which uses the same techniques as appendix B of [27]. Recall the Higgs branch decomposition (2.32) near 0,

$$
Z=\sum_{p=1}^{N_{f}}\left\{(x \bar{x})^{-\mathrm{i} m_{p}} \frac{\prod_{s \neq p}^{N_{f}} \gamma\left(-\mathrm{i} m_{s}+\mathrm{i} m_{p}\right)}{\prod_{s=1}^{N_{f}} \gamma\left(1+\mathrm{i} \widetilde{m}_{s}+\mathrm{i} m_{p}\right)} f_{p}^{(\mathrm{s})}(m, \widetilde{m}, x) f_{p}^{(\mathrm{s})}(m, \widetilde{m}, \bar{x})\right\},
$$

where the series (2.33) defining $f_{p}^{(\mathrm{s})}(x)$ converges for $|x|<1$. Similarly, the Higgs branch decomposition near $\infty$ involves series which converge for $|x|>1$. We wish to relate the two sets of holomorphic factors, or rather, their analytic continuation to a common domain. This is done through the integral representation (2.38) also given in (A.26) below, which converges away from the positive real axis. For $|x| \lessgtr 1$ we close the contour integral towards $\kappa \rightarrow \pm \infty$, enclosing either the poles at $\kappa+\mathrm{i} m_{p} \in \mathbb{Z}_{\geq 0}$ or the $N_{f}$ families of poles at $\kappa-\mathrm{i} \widetilde{m}_{s} \in \mathbb{Z}_{\leq 0}$ labeled by a flavour $s$. The first choice yields a single s-channel factor, while the second yields a sum of $N_{f}$ u-channel factors:

$$
\begin{aligned}
(-x)^{-\mathrm{i} m_{p}} f_{p}^{(\mathrm{s})}(x) & \stackrel{\operatorname{cont}}{=} \prod_{s=1}^{N_{f}}\left[\frac{\Gamma\left(1+\mathrm{i} m_{s}-\mathrm{i} m_{p}\right)}{\Gamma\left(-\mathrm{i} \widetilde{m}_{s}-\mathrm{i} m_{p}\right)}\right] \int_{-\mathrm{i} \infty}^{\mathrm{i} \infty} \frac{\mathrm{d} \kappa}{2 \pi \mathrm{i}} \frac{\prod_{s=1}^{N_{f}} \Gamma\left(-\mathrm{i} \widetilde{m}_{s}+\kappa\right)}{\prod_{s \neq p}^{N_{f}} \Gamma\left(1+\mathrm{i} m_{s}+\kappa\right)} \Gamma\left(-\kappa-\mathrm{i} m_{p}\right)(-x)^{\kappa} \\
& \stackrel{\operatorname{cont}}{=} \sum_{s=1}^{N_{f}} B_{p s}^{0}(-x)^{\mathrm{i} \widetilde{m}_{s}} f_{s}^{(\mathrm{u})}(x)=\sum_{s=1}^{N_{f}} D_{p} \check{B}_{p s}^{0} \widetilde{D}_{s}(-x)^{\mathrm{i} \widetilde{m}_{s}} f_{s}^{(\mathrm{u})}(x) .
\end{aligned}
$$

The transfer matrix $B_{p s}^{0}$ is the product of simpler matrices $D, \check{B}^{0}$ and $\widetilde{D}$ given in (A.28). It is also convenient to work with the s-channel factors $x^{-\mathrm{i} m_{p}} f_{p}^{(\mathrm{s})}(x)$, analytically continued with branch cuts on $(-\infty, 0] \cup[1,+\infty)$, and the $\mathrm{u}$-channel factors $x^{\mathrm{i} \widetilde{m}_{s}} f_{s}^{(\mathrm{u})}(x)$, with branch cuts along $(-\infty, 0] \cup[0,1]$, rather than with the factors appearing in (A.26), which all have branch cuts along $[0,1] \cup[1,+\infty)$. Using $(-x)^{\lambda}=e^{-\mathrm{i} \pi \epsilon \lambda} x^{\lambda}$ for $\epsilon=\operatorname{sign}(\operatorname{Im} x)$, we obtain

$$
x^{-\mathrm{i} m_{p}} f_{p}^{(\mathrm{s})}(x) \stackrel{\operatorname{cont}}{=} \sum_{s=1}^{N_{f}} B_{p s}^{\epsilon} x^{\mathrm{i} \widetilde{m}_{s}} f_{s}^{(\mathrm{u})}(x)=\sum_{s=1}^{N_{f}} D_{p} \check{B}_{p s}^{\epsilon} \widetilde{D}_{s} x^{\mathrm{i} \widetilde{m}_{s}} f_{s}^{(\mathrm{u})}(x),
$$

which only differs from (A.26) by a phase in $\check{B}^{\epsilon}$ :

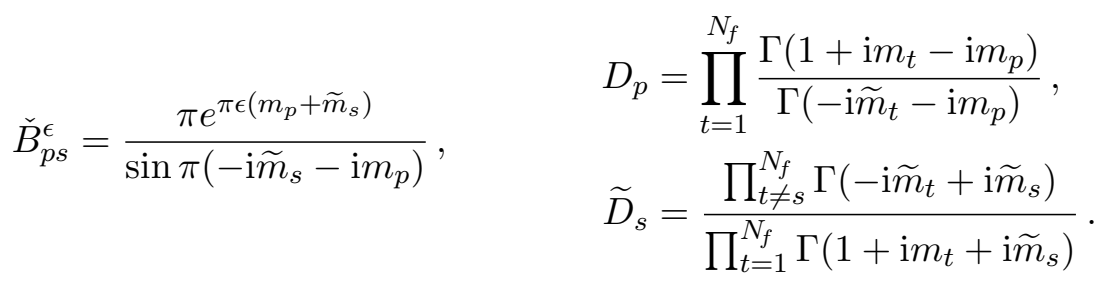


Through the matching of parameters $(2.20)$, the matrix $D \check{B}^{\epsilon} \widetilde{D}$ reproduces the appropriate braiding matrix (B.11) of [27]. This is expected since conformal blocks and vortex partition functions are already known explicitly to match.

The monodromy matrix around 1 is a product $M_{1}=B^{+}\left(B^{-}\right)^{-1}$ of braiding matrices. Since all $\check{B}_{p s}^{+}-\check{B}_{p s}^{-}=2 \pi \mathrm{i}$, the matrix $M_{1}-\mathrm{id}=D\left(\check{B}^{+}-\check{B}^{-}\right) \widetilde{D}\left(B^{-}\right)^{-1}$ has rank 1 . Thus, $M_{1}$ has the eigenvalue 1 with multiplicity $N_{f}-1$. This was used below (2.42).

Next, recall that the partition function of SQCD can be expressed as (2.57) in terms of derivatives of a product of SQED partition functions. This also holds for s-channel (and $\mathrm{u}$-channel) holomorphic factors (2.59), and we can analytically continue each SQED factor using (A.27):

$$
\begin{aligned}
& x^{-\sum_{j=1}^{N} \mathrm{i} m_{p_{j}}} f_{\{p\}}^{(\mathrm{s})}(x)=\left[\prod_{i<j} \frac{x_{i} \partial_{x_{i}}-x_{j} \partial_{x_{j}}}{-\mathrm{i} m_{p_{i}}+\mathrm{i} m_{p_{j}}} \prod_{j=1}^{N}\left[x_{j}^{-\mathrm{i} m_{p_{j}}} f_{p_{j}}^{(\mathrm{s})}\left(x_{j}\right)\right]\right]_{x_{j}=x} \\
& \stackrel{\text { cont }}{=}\left[\prod_{i<j} \frac{x_{i} \partial_{x_{i}}-x_{j} \partial_{x_{j}}}{-\mathrm{i} m_{p_{i}}+\mathrm{i} m_{p_{j}}} \prod_{j=1}^{N} \sum_{s_{j}=1}^{N_{f}}\left[D_{p_{j}} \check{B}_{p_{j} s_{j}}^{\epsilon} \widetilde{D}_{s_{j}} x_{j}^{\mathrm{i} \widetilde{m}_{s_{j}}} f_{s_{j}}^{(\mathrm{u})}\left(x_{j}\right)\right]\right]_{x_{j}=x} \\
& =\sum_{s_{1} \neq \cdots \neq s_{N}}\left[\prod_{j=1}^{N}\left[D_{p_{j}} \check{B}_{p_{j} s_{j}}^{\epsilon} \widetilde{D}_{s_{j}}\right] \prod_{i<j}\left[\frac{\mathrm{i} \widetilde{m}_{s_{i}}-\mathrm{i} \widetilde{m}_{s_{j}}}{-\mathrm{i} m_{p_{i}}+\mathrm{i} m_{p_{j}}}\right] x^{\left.\sum_{j=1}^{N} \mathrm{i} \widetilde{m}_{s_{j}} f_{\{s\}}^{(\mathrm{u})}(x)\right]}\right. \\
& =\sum_{1 \leq s_{1}<\cdots<s_{N} \leq N_{f}} B_{\{p\}\{s\}}^{\epsilon} x^{\sum_{j=1}^{N} \mathrm{i} \widetilde{m}_{s_{j}}} f_{\{s\}}^{(\mathrm{u})}(x),
\end{aligned}
$$

where $B_{\{p\}\{s\}}^{\epsilon}=D_{\{p\}} \check{B}_{\{p\}\{s\}}^{\epsilon} \widetilde{D}_{\{s\}}$ in terms of matrices given below, and another form of $B_{\{p\}\{s\}}^{\epsilon}$ is in (A.37). To get (A.31), we note that if $s_{i}=s_{j}$ for some $i \neq j$, the differential operators $x_{i} \partial_{x_{i}}$ and $x_{j} \partial_{x_{j}}$ act identically on the product of SQED factors (once $x_{i}$ and $x_{j}$ are set to $x$ ), hence the term does not contribute. After restricting ourselves to terms with all $s_{i}$ distinct, we can safely extract the product of $\mathrm{i} \widetilde{m}_{s_{i}}-\mathrm{i} \widetilde{m}_{s_{j}}$ to convert SQED u-channel factors to the SQCD one. The last step sums over permutations of the $s_{i}$, to collect terms with the same factor, labeled by the set $\{s\}$. The various ingredients are two diagonal matrices,

$$
D_{\{p\}}=\frac{\prod_{j=1}^{N} D_{p_{j}}}{\prod_{i<j}\left(-\mathrm{i} m_{p_{i}}+\mathrm{i} m_{p_{j}}\right)}, \quad \widetilde{D}_{\{s\}}=\prod_{i<j}\left(\mathrm{i} \widetilde{m}_{s_{i}}-\mathrm{i} \widetilde{m}_{s_{j}}\right) \prod_{j=1}^{N} \widetilde{D}_{s_{j}},
$$

and the $N$-th wedge power $\check{B}_{\{p\}\{s\}}^{\epsilon}$ of the SQED matrix $\check{B}_{p s}^{\epsilon}$ :

$$
\begin{gathered}
\check{B}_{\{p\}\{s\}}^{\epsilon}=\sum_{\sigma \in S_{N}}(-1)^{\sigma} \prod_{j=1}^{N} \check{B}_{p_{j} s_{\sigma(j)}}^{\epsilon}=\sum_{\sigma \in S_{N}}(-1)^{\sigma} \prod_{j=1}^{N} \frac{\pi e^{\pi \epsilon\left(m_{p_{j}}+\widetilde{m}_{s_{\sigma(j)}}\right)}}{\sin \pi\left(-\mathrm{i} \widetilde{m}_{s_{\sigma(j)}}-\mathrm{i} m_{p_{j}}\right)} \\
=\int \frac{\mathrm{d} \kappa_{1}}{2 \mathrm{i}} \cdots \frac{\mathrm{d} \kappa_{N}}{2 \mathrm{i}} \frac{\prod_{i<j} \sin \pi\left(\kappa_{i}-\kappa_{j}\right) \sin \pi\left(\mathrm{i} \widetilde{m}_{s_{i}}-\mathrm{i} \widetilde{m}_{s_{j}}\right)}{\prod_{i, j=1}^{N} \sin \pi\left(\kappa_{j}+\mathrm{i} \widetilde{m}_{s_{i}}\right)} \frac{\pi e^{\pi \epsilon\left(m_{p_{j}}+\widetilde{m}_{s_{j}}\right)}}{\sin \pi\left(\kappa_{j}-\mathrm{i} m_{p_{j}}\right)} \\
=\frac{\pi^{N} e^{\pi \epsilon \sum_{j=1}^{N}\left(m_{p_{j}}+\widetilde{m}_{s_{j}}\right)} \prod_{i<j} \sin \pi\left(\mathrm{i} \widetilde{m}_{s_{i}}-\mathrm{i} \widetilde{m}_{s_{j}}\right) \prod_{i<j} \sin \pi\left(\mathrm{i} m_{p_{i}}-\mathrm{i} m_{p_{j}}\right)}{\prod_{i, j} \sin \pi\left(-\mathrm{i} \widetilde{m}_{s_{i}}-\mathrm{i} m_{p_{j}}\right)} .
\end{gathered}
$$


The $\mathrm{d} \kappa_{j}$ contours in (A.35) are each a pair of vertical lines $\frac{1}{2}-\mathrm{i} \infty \rightarrow \frac{1}{2}+\mathrm{i} \infty$ and $\mathrm{i} \infty \rightarrow$ $-\mathrm{i} \infty$, surrounding poles at $\kappa_{j}=-\mathrm{i} \widetilde{m}_{s_{\sigma(j)}}$. Convergence is guaranteed since the integrand decreases exponentially as $\operatorname{Im} \kappa \rightarrow \pm \infty$ (for $-1 \leq \epsilon \leq 1$ ). If two $\sigma(j)$ are equal, the numerator sines lead to a vanishing residue. Otherwise, the first fraction completely cancels and we retrieve (A.34). Next, we note that the integrand has period 1, hence the contour can be replaced by $-\frac{1}{2}-\mathrm{i} \infty \rightarrow-\frac{1}{2}+\mathrm{i} \infty$ and $\mathrm{i} \infty \rightarrow-\mathrm{i} \infty$, which surrounds poles at $\kappa_{j}=\mathrm{i} m_{p_{j}}$, with a factor of $(-1)^{N}$ to account for the orientation of the contour. This yields the last expression.

All in all, the matrix relating s-channel and $\mathrm{u}$-channel factors in (A.32) is

$$
B_{\{p\}\{s\}}^{\epsilon}=\prod_{p \in\{p\}} \frac{e^{\pi \epsilon m_{p}} \prod_{t \notin\{p\}}^{N_{f}} \Gamma\left(1+\mathrm{i} m_{t}-\mathrm{i} m_{p}\right)}{\prod_{u \notin\{s\}}^{N_{f}} \Gamma\left(-\mathrm{i} m_{p}-\mathrm{i} \widetilde{m}_{u}\right)} \prod_{s \in\{s\}} \frac{e^{\pi \epsilon \widetilde{m}_{s}} \prod_{u \notin\{s\}}^{N_{f}} \Gamma\left(\mathrm{i} \widetilde{m}_{s}-\mathrm{i} \widetilde{m}_{u}\right)}{\prod_{t \notin\{p\}}^{N_{f}} \Gamma\left(1+\mathrm{i} m_{t}+\mathrm{i} \widetilde{m}_{s}\right)} .
$$

\section{A.3.2 Toda CFT braiding matrices}

So far in this appendix, we have manipulated gauge theory factors only. For the gauge theory/Toda CFT correspondence to hold, those should be equal to conformal blocks multiplied by the factor $z^{\gamma_{0}}(1-z)^{\gamma_{1}}$ appearing in (2.51). We will show that the braiding matrix relating s-channel and u-channel conformal blocks is $\mathbf{B}_{P S}^{\epsilon}=e^{\mathrm{i} \pi \epsilon \gamma_{1}} B_{P S}^{\epsilon}$, where we denote $P=\{p\}$ and $S=\{s\}$. This implies in particular that all monodromy matrices on the gauge theory side and the Toda CFT side match, thus establishes the correspondence for SQCD, up to a factor fixed in appendix A.2.

The braiding matrix $\mathbf{B}_{P S}^{\epsilon}$ is defined by

$$
\mathcal{F}_{\alpha_{0}-b h_{P}}^{(\mathrm{s})}\left[\begin{array}{cc}
\hat{m} & -b \omega_{N} \\
\alpha_{\infty} & \alpha_{0}
\end{array}\right](x)=\sum_{\substack{S \subseteq \llbracket 1, N_{f} \rrbracket \\
\# S=N}} \mathbf{B}_{P S}^{\epsilon}\left[\begin{array}{cc}
\hat{m} & -b \omega_{N} \\
\alpha_{\infty} & \alpha_{0}
\end{array}\right] \mathcal{F}_{\alpha_{\infty}-b h_{S}}^{(\mathrm{u})}\left[\begin{array}{cc}
\hat{m} & -b \omega_{N} \\
\alpha_{\infty} & \alpha_{0}
\end{array}\right](x)
$$

where $\hat{m}=(\varkappa+N b) h_{1}$, and we will often decompose $\alpha_{0}=Q-\mathrm{i} a_{0}, \alpha_{\infty}=Q-\mathrm{i} a_{\infty}$. Using the dictionary $\gamma_{1}=\frac{N}{N_{f}}(b \varkappa+N b)-N\left(1+b^{2}\right), a_{0}=\frac{1}{b} \sum_{t=1}^{N_{f}} m_{t} h_{t}, a_{\infty}=\frac{1}{b} \sum_{t=1}^{N_{f}} \widetilde{m}_{t} h_{t}$, and $\varkappa=\frac{1}{b} \sum_{t=1}^{N_{f}}\left(1+m_{t}+\widetilde{m}_{t}\right)$ of $(2.52)$, we wish to prove that

$$
\begin{aligned}
& \mathbf{B}_{P S}^{\epsilon}\left[\begin{array}{cc}
(\varkappa+N b) h_{1} & -b \omega_{N} \\
Q-\mathrm{i} a_{\infty} & Q-\mathrm{i} a_{0}
\end{array}\right]=e^{\mathrm{i} \pi \epsilon \gamma_{1}} B_{P S}^{\epsilon} \\
&=e^{-\mathrm{i} \pi \epsilon \frac{N\left(N_{f}-N\right)}{N_{f}} b^{2}} \prod_{p \in P} \frac{e^{\pi \epsilon b\left\langle a_{0}, h_{p}\right\rangle} \prod_{t \notin P}^{N_{f}} \Gamma\left(1+b\left\langle\mathrm{i} a_{0}, h_{t}-h_{p}\right\rangle\right)}{\prod_{u \notin S}^{N_{f}} \Gamma\left(1-\frac{b \varkappa}{N_{f}}-b\left\langle\mathrm{i} a_{0}, h_{p}\right\rangle-b\left\langle\mathrm{i} a_{\infty}, h_{u}\right\rangle\right)} \\
& \prod_{s \in S} \frac{e^{\pi \epsilon b\left\langle a_{\infty}, h_{s}\right\rangle} \prod_{u \notin S}^{N_{f}} \Gamma\left(b\left\langle\mathrm{i} a_{\infty}, h_{s}-h_{u}\right\rangle\right)}{\prod_{t \notin P}^{N_{f}} \Gamma\left(\frac{b \varkappa}{N_{f}}+b\left\langle\mathrm{i} a_{0}, h_{t}\right\rangle+b\left\langle\mathrm{i} a_{\infty}, h_{s}\right\rangle\right)} .
\end{aligned}
$$

We proceed by induction on $N$. For $N=1$, the Toda CFT braiding matrix is known, as mentioned below (A.28), and matches with the gauge theory transfer matrix, thus (A.39) holds. From here on, we assume (A.39) for a given $N$. In particular, the s-channel conformal blocks are given by the gauge theory holomorphic factors (A.76) for 
that value of $N$, because conformal blocks are uniquely determined by their monodromy exponents at $\{0,1, \infty\}$ and the braiding matrix around 1.

We first deduce the fusion of $V_{-b h_{1}}$ and $V_{-b \omega_{N}}$ into $V_{-b \omega_{N+1}}$,

$$
\mathcal{F}_{-b \omega_{N+1}}^{(\mathrm{t})}\left[\begin{array}{cc}
-b h_{1} & -b \omega_{N} \\
2 Q-\alpha_{0}+b h_{P} & \alpha_{0}
\end{array}\right]=\sum_{p \in P} \mathbf{F}_{p, P}\left[\alpha_{0}\right] \mathcal{F}_{\alpha_{0}-b h_{P \backslash\{p\}}}^{(\mathrm{s})}\left[\begin{array}{cc}
-b h_{1} & -b \omega_{N} \\
2 Q-\alpha_{0}+b h_{P} & \alpha_{0}
\end{array}\right],
$$

which must have the monodromy $e^{2 \pi \mathrm{i}\left[\Delta\left(-b \omega_{N}\right)+\Delta\left(-b h_{1}\right)-\Delta\left(-b \omega_{N+1}\right)\right]}=e^{-2 \pi \mathrm{i}\left[N\left(b^{2}+1\right)+b^{2} N / N_{f}\right]}$ around $x=1$. We shall prove that the fusion coefficients

$$
\mathbf{F}_{p, P}\left[\alpha_{0}\right]=\frac{\Gamma\left((N+1)\left(1+b^{2}\right)\right)}{\Gamma\left(1+b^{2}\right)} \prod_{t \in P \backslash\{p\}}\left[\frac{\Gamma\left(b\left\langle Q-\alpha_{0}, h_{t}-h_{p}\right\rangle\right)}{\Gamma\left(1+b^{2}+b\left\langle Q-\alpha_{0}, h_{t}-h_{p}\right\rangle\right)}\right]
$$

give this monodromy, and are normalized so that the dominant power of $1-x$ has a coefficient 1.

Braid $V_{-b \omega_{N}}$ and $V_{-b h_{1}}$ in the right-hand side of (A.40) using (A.39) with $P \rightarrow P \backslash\{p\}$, $\varkappa \rightarrow-(N+1) b, \mathrm{i} a_{\infty} \rightarrow-\mathrm{i} a_{0}-b h_{P}$ and $S \rightarrow P \backslash\{s\}$ for some $s \in P\left(h_{P}-h_{S}\right.$ must be a weight of the fundamental representation, because of $\left.V_{-b h_{1}}\right)$ :

$$
\begin{aligned}
\sum_{p \in P} \mathbf{F}_{p, P}\left[\alpha_{0}\right] \mathbf{B}_{P \backslash\{p\}, P \backslash\{s\}}^{\epsilon}\left[\begin{array}{cc}
-b h_{1} & -b \omega_{N} \\
2 Q-\alpha_{0}+b h_{P} & \alpha_{0}
\end{array}\right] \\
=e^{-\mathrm{i} \pi \epsilon \frac{N}{N_{f}} b^{2}} \sum_{p \in P} e^{\pi \epsilon b\left\langle a_{0}, h_{s}-h_{p}\right\rangle} \frac{\prod_{t \in P \backslash\{s\}} \sin \pi\left(1+b^{2}+b\left\langle\mathrm{i} a_{0}, h_{t}-h_{p}\right\rangle\right)}{\prod_{t \in P \backslash\{p\}} \sin \pi\left(b\left\langle\mathrm{i} a_{0}, h_{t}-h_{p}\right\rangle\right)} \\
\\
\quad \cdot \frac{\Gamma\left((N+1)\left(1+b^{2}\right)\right)}{\Gamma\left(1+b^{2}\right)} \prod_{t \in P \backslash\{s\}}\left[\frac{\Gamma\left(b\left\langle\mathrm{i} a_{0}, h_{s}-h_{t}\right\rangle\right)}{\Gamma\left(1+b^{2}+b\left\langle\mathrm{i} a_{0}, h_{s}-h_{t}\right\rangle\right)}\right] \\
=e^{-\mathrm{i} \pi \epsilon\left[\frac{N}{N_{f}} b^{2}+N\left(1+b^{2}\right)\right]} \mathbf{F}_{s, P}\left[2 Q-\alpha_{0}+b h_{P}\right] .
\end{aligned}
$$

We have used

$$
\begin{aligned}
& \sum_{p \in P} e^{\pi \epsilon b\left\langle a_{0}, h_{s}-h_{p}\right\rangle} \frac{\prod_{t \in P \backslash\{s\}} \sin \pi\left(1+b^{2}+b\left\langle\mathrm{i} a_{0}, h_{t}-h_{p}\right\rangle\right)}{\prod_{t \in P \backslash\{p\}} \sin \pi\left(b\left\langle\mathrm{i} a_{0}, h_{t}-h_{p}\right\rangle\right)} \\
& =\int \frac{\mathrm{d} \kappa}{2 \mathrm{i}} \frac{e^{\pi \epsilon\left(b\left\langle a_{0}, h_{s}\right\rangle-\mathrm{i} \kappa\right)} \prod_{t \in P \backslash\{s\}} \sin \pi\left(1+b^{2}+\mathrm{i} m_{t}+\kappa\right)}{\prod_{t \in P} \sin \pi\left(\mathrm{i} m_{t}+\kappa\right)}=e^{-\mathrm{i} \pi \epsilon N\left(1+b^{2}\right)},
\end{aligned}
$$

where the contour surrounds the rectangle $\operatorname{Re} \kappa \in[0,1], \operatorname{Im} \kappa \in(-\infty, \infty)$. Summing over poles yields the sum over $p \in P$ in the first line. The integrals over the lines $1-\mathrm{i} \infty \rightarrow$ $1+\mathrm{i} \infty$ and $\mathrm{i} \infty \rightarrow-\mathrm{i} \infty$ cancel because the integrand is 1 -periodic, and the integrals over $1+\mathrm{i} \infty \rightarrow \mathrm{i} \infty$ and $-\mathrm{i} \infty \rightarrow 1-\mathrm{i} \infty$ yield 0 and $e^{-\mathrm{i} \pi \epsilon N\left(1+b^{2}\right)}$ in some order.

In (A.43), we have only done one braiding move, not a full monodromy (two braiding moves). However, the combination of $\mathrm{u}$-channel conformal blocks is identical to (A.40) after changing $\mathrm{i} a_{0} \rightarrow \mathrm{i} a_{\infty}=-\mathrm{i} a_{0}-b h_{P}$, thus, by symmetry, braiding once more to reach the s-channel yields the same phase factor. Therefore, (A.40) has the announced monodromy around $x=1$. 
There remains to fix the normalization. We evaluate at $x=1$ the explicit expression (A.76) of s-channel conformal blocks which appear in (A.40), after removing a power of $(1-x)$,

$$
\begin{aligned}
& {\left[(1-x)^{-N\left(b^{2}+1\right)-\frac{N}{N_{f}} b^{2}}\right.}\left.\mathcal{F}_{\alpha_{0}-b h_{P \backslash\{p\}}}^{(\mathrm{s})}\left[\begin{array}{cc}
-b h_{1} & -b \omega_{N} \\
2 Q-\alpha_{0}+b h_{P} & \alpha_{0}
\end{array}\right](x)\right]_{x=1} \\
&=\sum_{\substack{k: P \rightarrow \mathbb{Z}_{\geq 0} \\
k_{p}=0}}(-1)^{\sum_{s \in P} k_{s}} \prod_{s, t \in P} \frac{\left(1+b^{2}+b\left\langle\mathrm{i} a_{0}, h_{t}-h_{s}\right\rangle\right)_{k_{s}}}{\left(b\left\langle\mathrm{i} a_{0}, h_{t}-h_{s}\right\rangle-k_{t}+\delta_{t p}\right)_{k_{s}}} .
\end{aligned}
$$

This only depends on the $\left\langle\mathrm{i} a_{0}, h_{t}\right\rangle$ with $t \in P$, and does not depend on $N_{f}$. We can thus take $N_{f}=N+1$, in which case $-b \omega_{N}=b h_{N_{f}}$ and the fusion is a special case of equation (B.14) of [27], where the normalization is known to be (A.41).

We are now ready to find the braiding matrix of $V_{-b \omega_{N+1}}$ with $V_{\hat{m}}$ (where $\hat{m}=(\varkappa+$ $\left.(N+1) b) h_{1}\right)$. This braiding, followed by writing $V_{-b \omega_{N+1}}$ as the fusion of $V_{-b h_{1}}$ and $V_{-b \omega_{N}}$, is equivalent to performing the fusion step first, then braiding each of $V_{-b h_{1}}$ and $V_{-b \omega_{N}}$ in turn around the semi-degenerate operator. The equivalence is encoded as a pentagon identity: for any $(N+1)$-element sets of flavours $P$ and $S$, and for $s \in S$,

$$
\begin{aligned}
\mathbf{B}_{P S}^{\epsilon}\left[\begin{array}{cc}
\hat{m} & -b \omega_{N+1} \\
\alpha_{\infty} & \alpha_{0}
\end{array}\right] \mathbf{F}_{s, S}\left[2 Q-\alpha_{\infty}\right] & \\
& =\sum_{p \in P} \mathbf{F}_{p, P}\left[\alpha_{0}\right] \mathbf{B}_{p s}^{\epsilon}\left[\begin{array}{cc}
\hat{m} & -b h_{1} \\
\alpha_{\infty} & \alpha_{0}-b h_{P \backslash\{p\}}
\end{array}\right] \mathbf{B}_{P \backslash\{p\}, S \backslash\{s\}}^{\epsilon}\left[\begin{array}{cc}
\hat{m} & -b \omega_{N} \\
\alpha_{\infty}-b h_{s} & \alpha_{0}
\end{array}\right]
\end{aligned}
$$

As a consistency check, we compute a slightly more general right-hand side, with $S \backslash\{s\}$ replaced by any $N$-element subset $S^{\prime}$ of $\llbracket 1, N_{f} \rrbracket$. This altered right-hand side must vanish whenever $s \in S^{\prime}$. After extracting factors independent of $p$ in (A.48) below, we will obtain a sum over $p$ of products of sines, which is a sum of residues:

$$
\begin{aligned}
& \sum_{p \in P} \frac{\prod_{u \in S^{\prime}} \frac{1}{\pi} \sin \pi\left(\frac{b \varkappa}{N_{f}}+b\left\langle\mathrm{i} a_{0}, h_{p}\right\rangle+b\left\langle\mathrm{i} a_{\infty}, h_{u}\right\rangle+b^{2} \delta_{u s}\right)}{\frac{1}{\pi} \sin \pi\left(\frac{b \varkappa}{N_{f}}+b^{2}+b\left\langle\mathrm{i} a_{0}, h_{p}\right\rangle+b\left\langle\mathrm{i} a_{\infty}, h_{s}\right\rangle\right) \prod_{t \in P \backslash\{p\}} \frac{1}{\pi} \sin \pi\left(b\left\langle\mathrm{i} a_{0}, h_{t}-h_{p}\right\rangle\right)} \\
& =-\sum_{p \in P} \operatorname{res}_{\kappa=\mathrm{i} m_{p}} \frac{\prod_{u \in S^{\prime}} \frac{1}{\pi} \sin \pi\left(1+b^{2} \delta_{u s}+\kappa+\mathrm{i} \widetilde{m}_{u}\right)}{\frac{1}{\pi} \sin \pi\left(1+b^{2}+\kappa+\mathrm{i} \widetilde{m}_{s}\right) \prod_{t \in P} \frac{1}{\pi} \sin \pi\left(\mathrm{i} m_{t}-\kappa\right)} \\
& =\frac{\prod_{u \in S^{\prime}} \frac{1}{\pi} \sin \pi\left(b^{2} \delta_{u s}+\mathrm{i} \widetilde{m}_{u}-b^{2}-\mathrm{i} \widetilde{m}_{s}\right)}{\prod_{t \in P} \frac{1}{\pi} \sin \pi\left(\mathrm{i} m_{t}+\mathrm{i} \widetilde{m}_{s}+1+b^{2}\right)} .
\end{aligned}
$$

This sum of residues is equal to the residue at $\kappa=-1-b^{2}-\mathrm{i} \widetilde{m}_{s}$ written in the last line, because the function of $\kappa$ is 1 -periodic and vanishes at $\kappa \rightarrow \pm \mathrm{i} \infty$, hence the integral over the boundary of $[0,1] \times(-\infty, \infty)$ vanishes. As expected, the result is 0 when $s \in S^{\prime}$ (take $u=s)$. It is otherwise a product of sines, and we get in that case the last equality below 
(denoting $S=S^{\prime} \cup\{s\}$ ):

$$
\begin{aligned}
& \sum_{p \in P} \mathbf{F}_{p, P}\left[\alpha_{0}\right] \mathbf{B}_{p s}^{\epsilon}\left[\begin{array}{cc}
\hat{m} & -b h_{1} \\
\alpha_{\infty} & \alpha_{0}-b h_{P \backslash\{p\}}
\end{array}\right] \mathbf{B}_{P \backslash\{p\}, S^{\prime}}^{\epsilon}\left[\begin{array}{cc}
\hat{m} & -b \omega_{N} \\
\alpha_{\infty}-b h_{s} & \alpha_{0}
\end{array}\right] \\
& =\frac{e^{-\mathrm{i} \pi \epsilon \frac{(N+1)\left(N_{f}-N-1\right)}{N_{f}} b^{2}-\mathrm{i} \pi \epsilon \delta_{s \in S^{\prime}} b^{2}+\pi \epsilon b\left\langle a_{0}, h_{P}\right\rangle+\pi \epsilon b\left\langle a_{\infty}, h_{s}+h_{S^{\prime}}\right\rangle} \prod_{u \neq s}^{N_{f}} \Gamma\left(b\left\langle\mathrm{i} a_{\infty}, h_{s}-h_{u}\right\rangle\right)}{\prod_{t=1}^{N_{f}} \Gamma\left(\frac{b \varkappa}{N_{f}}+b^{2} \delta_{t \in P}+b\left\langle\mathrm{i} a_{0}, h_{t}\right\rangle+b\left\langle\mathrm{i} a_{\infty}, h_{s}\right\rangle\right)} \\
& \cdot \prod_{t \in P} \frac{\prod_{v \notin P}^{N_{f}} \Gamma\left(1+b\left\langle\mathrm{i} a_{0}, h_{v}-h_{t}\right\rangle\right)}{\prod_{w \notin S^{\prime}}^{N_{f}} \Gamma\left(1-\frac{b \varkappa}{N_{f}}-b\left\langle\mathrm{i} a_{0}, h_{t}\right\rangle-b\left\langle\mathrm{i} a_{\infty}, h_{w}\right\rangle-b^{2} \delta_{s w}\right)} \\
& \cdot \frac{\Gamma\left((N+1)\left(1+b^{2}\right)\right)}{\Gamma\left(1+b^{2}\right)} \prod_{u \in S^{\prime}} \frac{\prod_{w \notin S^{\prime}}^{N_{f}} \Gamma\left(b\left\langle\mathrm{i} a_{\infty}, h_{u}-h_{w}\right\rangle+b^{2} \delta_{s u}-b^{2} \delta_{s w}\right)}{\prod_{v \notin P}^{N_{f}} \Gamma\left(\frac{b \varkappa}{N_{f}}+b\left\langle\mathrm{i} a_{0}, h_{v}\right\rangle+b\left\langle\mathrm{i} a_{\infty}, h_{u}\right\rangle+b^{2} \delta_{u s}\right)} \\
& \cdot \sum_{p \in P} \frac{\prod_{u \in S^{\prime}} \frac{1}{\pi} \sin \pi\left(\frac{b \varkappa}{N_{f}}+b\left\langle\mathrm{i} a_{0}, h_{p}\right\rangle+b\left\langle\mathrm{i} a_{\infty}, h_{u}\right\rangle+b^{2} \delta_{u s}\right)}{\frac{1}{\pi} \sin \pi\left(\frac{b \varkappa}{N_{f}}+b^{2}+b\left\langle\mathrm{i} a_{0}, h_{p}\right\rangle+b\left\langle\mathrm{i} a_{\infty}, h_{s}\right\rangle\right) \prod_{t \in P \backslash\{p\}} \frac{1}{\pi} \sin \pi\left(b\left\langle\mathrm{i} a_{0}, h_{t}-h_{p}\right\rangle\right)} \\
& \stackrel{s \notin S^{\prime}}{=} e^{-\mathrm{i} \pi \epsilon \frac{(N+1)\left(N_{f}-N-1\right)}{N_{f}} b^{2}} \prod_{t \in P} \frac{e^{\pi \epsilon b\left\langle a_{0}, h_{t}\right\rangle} \prod_{v \notin P}^{N_{f}} \Gamma\left(1+b\left\langle\mathrm{i} a_{0}, h_{v}-h_{t}\right\rangle\right)}{\prod_{w \notin S}^{N_{f}} \Gamma\left(1-\frac{b \varkappa}{N_{f}}-b\left\langle\mathrm{i} a_{0}, h_{t}\right\rangle-b\left\langle\mathrm{i} a_{\infty}, h_{w}\right\rangle\right)} \\
& \cdot \prod_{u \in S} \frac{e^{\pi \epsilon b\left\langle a_{\infty}, h_{u}\right\rangle} \prod_{w \notin S}^{N_{f}} \Gamma\left(b\left\langle\mathrm{i} a_{\infty}, h_{u}-h_{w}\right\rangle\right)}{\prod_{v \notin P}^{N_{f}} \Gamma\left(\frac{b \varkappa}{N_{f}}+b\left\langle\mathrm{i} a_{0}, h_{v}\right\rangle+b\left\langle\mathrm{i} a_{\infty}, h_{u}\right\rangle\right)} \\
& \cdot \frac{\Gamma\left((N+1)\left(1+b^{2}\right)\right)}{\Gamma\left(1+b^{2}\right)} \prod_{u \in S \backslash\{s\}} \frac{\Gamma\left(b\left\langle\mathrm{i} a_{\infty}, h_{s}-h_{u}\right\rangle\right)}{\Gamma\left(1+b^{2}+b\left\langle\mathrm{i} a_{\infty}, h_{s}-h_{u}\right\rangle\right)} .
\end{aligned}
$$

We recognize in the last line the fusion coefficient $\mathbf{F}_{s, S}\left[2 Q-\alpha_{\infty}\right]$. What remains is the braiding matrix of $V_{-b \omega_{N+1}}$ with $V_{\hat{m}}$, which we check to be (A.38) with $N \rightarrow N+1$. This concludes the induction, and the proof of the relation between conformal blocks and vortex partition functions for SQCD. Together with the equality (A.22) of constant factors, checked in appendix A.2, this establishes the relation (2.51) between the partition function of an SQCD surface operator and the appropriate correlator in the Toda CFT.

\section{A.4 Fusion rules}

We provide here the fusion rules between various pairs of vertex operators, in particular the fusion (A.61) of two semi-degenerate operators, and the fusion (A.73) of a semi-degenerate operator with a fully-degenerate operator labeled by an arbitrary representation of $A_{N_{f}-1}$. We propose that operators resulting from the latter fusion appear with multiplicity (A.74) in the fusion of two generic operators.

Null vectors among $W_{N_{f}}$ descendants of a fully degenerate vertex operator $V_{-b \omega-\omega^{\prime} / b}$ constrain its three-point function with arbitrary vertex operators $V_{\alpha}$ and $V_{\beta}$. Namely, the three point function vanishes unless $\alpha+\beta=2 Q+b h+h^{\prime} / b$ for some weights $h$ of $\mathcal{R}(\omega)$ 
and $h^{\prime}$ of $\mathcal{R}\left(\omega^{\prime}\right)$. This results in the fusion rule

$$
V_{\alpha} \times V_{-b \omega-\omega^{\prime} / b}=\sum_{h \in \mathcal{R}(\omega)} \sum_{h^{\prime} \in \mathcal{R}\left(\omega^{\prime}\right)} V_{\alpha-b h-h^{\prime} / b}
$$

with outgoing momenta $\alpha-b h-h^{\prime} / b=2 Q-\beta$ : the degenerate operator shifts the incoming momentum by $-b h-h^{\prime} / b$. Each operator $V_{\alpha-b h-h^{\prime} / b}$ appears in (A.50) with a multiplicity equal to the product of the multiplicity of $h$ in $\mathcal{R}(\omega)$ and that of $h^{\prime}$ in $\mathcal{R}\left(\omega^{\prime}\right)$. Henceforth, we take $\omega^{\prime}=0$, thus $h^{\prime}=0$.

Later in this appendix, we find that the fusion (A.73) of a semi-degenerate operator $V_{\varkappa h_{1}}$ with $V_{-b \omega}$ only allows some of the shifts -bh of (A.50). Let us first describe the case $\omega=h_{1}$ based on [27, appendix B]: the fusion of $-\varkappa h_{N_{f}}$ and $-b h_{1}$ yields the momenta $-(\varkappa+b) h_{N_{f}}$ and $-\varkappa h_{N_{f}}-b h_{1}$. After the Weyl rotation $\left(12 \cdots N_{f}\right)$, we get

$$
V_{\varkappa h_{1}} \times V_{-b h_{1}}=V_{\varkappa h_{1}-b h_{1}}+V_{\varkappa h_{1}-b h_{2}} .
$$

The s-channel expansion of $\left\langle V_{\alpha_{\infty}}(\infty) V_{\varkappa h_{1}}(1) V_{-b h_{1}}(x, \bar{x}) V_{\alpha_{0}}(0)\right\rangle$ involves $N_{f}$ products of holomorphic and antiholomorphic conformal blocks. The t-channel expansion only features two momenta (A.51), and takes the form

$$
|1-z|^{2\left[\Delta\left(\varkappa h_{1}-b h_{1}\right)-\Delta\left(\varkappa h_{1}\right)-\Delta\left(-b h_{1}\right)\right]}(\cdots)+|1-z|^{2\left[\Delta\left(\varkappa h_{1}-b h_{2}\right)-\Delta\left(\varkappa h_{1}\right)-\Delta\left(-b h_{1}\right)\right]}(\cdots)
$$

where $(\cdots)$ are series in powers of $(1-z)$ and $(1-\bar{z})$. The first series factorizes as the product of a holomorphic and an anti-holomorphic conformal blocks, multiplied by $C_{-b h_{1}, \varkappa h_{1}}^{\varkappa h_{1}-b h_{1}} C\left(\alpha_{0}, \alpha_{\infty},(\varkappa-b) h_{1}\right)$. The second does not, but can be written non-canonically as a sum of $N_{f}-1$ products of the same form. This multiplicity implies that the fusion $V_{\alpha_{0}}$ and $V_{\alpha_{\infty}}$ includes $N_{f}-1$ copies of the representations of the $W_{N_{f}}$ algebra generated by $V_{\varkappa h_{1}-b h_{2}}$, while it only includes one copy of the representation generated by any semidegenerate operator $V_{\varkappa h_{1}}$. We generalize the statement to all momenta of the form $\varkappa h_{1}-b \omega$ in (A.74).

\section{A.4.1 Fusion of two semi-degenerate operators}

To reach more complicated degenerate operators, we first find which momenta result from the fusion of two semi-degenerate momenta $-\varkappa h_{N_{f}}$ and $\lambda h_{1}$. In principle, one could write null vectors descending from $V_{-\varkappa h_{N_{f}}}$ and $V_{\lambda h_{1}}$ for a given $N_{f}$ and, through those, constrain the momenta which arise in the OPE. Such constraints are polynomial in the momenta, and any constraint shown for generic $(b, \varkappa, \lambda)$ must hold for all $(b, \varkappa, \lambda)$ by continuity: in other words, fusion rules for more specific momenta can only become more restrictive. We are thus free to assume that $(b, \varkappa, \lambda)$ is generic.

Since null vectors are very difficult to write down for general $N_{f}$, we use a different route: the braiding matrix relating the s-channel $(x \rightarrow 0)$ and $\mathrm{u}$-channel $(x \rightarrow \infty)$ conformal blocks of $\left\langle V_{\lambda h_{1}}(\infty) V_{-\varkappa h_{N_{f}}}(1) V_{-b h_{1}}(x, \bar{x}) V_{\alpha_{0}}(0)\right\rangle$ should only lead to u-channel conformal blocks with internal momenta $\lambda h_{1}-b h_{1}$ and $\lambda h_{1}-b h_{2}$, and all other components must vanish. Specifically, we take $\alpha_{2}=2 Q-\lambda h_{1}, \hat{m}=-\varkappa h_{N_{f}}, \mu=-b h_{1}$ and $\alpha_{1}=2 Q-\alpha+b h_{l}$ 
in equation (B.12) of [27]:

$$
\begin{array}{r}
\mathcal{F}_{2 Q-\alpha}^{(\mathrm{s})}\left[\begin{array}{cc}
-\varkappa h_{N_{f}} & -b h_{1} \\
\lambda h_{1} & 2 Q-\alpha+b h_{l}
\end{array}\right](x)=\sum_{k=1}^{N_{f}} e^{i \pi \epsilon\left(\phi_{k l}-b \varkappa / N_{f}\right)} \prod_{j \neq l} \frac{\Gamma\left(1+b^{2}+b\left\langle\alpha-Q, h_{j}-h_{l}\right\rangle\right)}{\Gamma\left(1+b^{2}-\phi_{k j}\right)} \\
\cdot \prod_{j \neq k} \frac{\Gamma\left(b\left\langle\lambda h_{1}-Q, h_{j}-h_{k}\right\rangle\right)}{\Gamma\left(\phi_{j l}\right)} \mathcal{F}_{\lambda h_{1}-b h_{k}}^{(\mathrm{u})}\left[\begin{array}{cc}
-\varkappa h_{N_{f}} & -b h_{1} \\
\lambda h_{1} & 2 Q-\alpha+b h_{l}
\end{array}\right](x)
\end{array}
$$

where

$$
\phi_{s t}=b\left\langle-\varkappa h_{N_{f}}, h_{1}\right\rangle+b\left\langle\lambda h_{1}-Q, h_{s}\right\rangle-b\left\langle\alpha-Q, h_{t}\right\rangle .
$$

The coefficient must vanish for all $k \notin\{1,2\}$ and all $l$, hence one of the denominator Gamma functions must have a non-positive integer argument:

$$
\forall k \in \llbracket 3, N_{f} \rrbracket \forall l \in \llbracket 1, N_{f} \rrbracket-\phi_{j l} \in \mathbb{Z}_{\geq 0} \text { or } \phi_{k j}-1-b^{2}=\phi_{(k-1) j} \in \mathbb{Z}_{\geq 0} .
$$

If for each $1 \leq s \leq N_{f}$ one had $\phi_{p_{s} s}=n_{s}$ for some integers $1 \leq p_{s} \leq N_{f}$ and $n_{s}$, then summing over $s$ would yield

$$
0=\sum_{s=1}^{N_{f}} b\left\langle\alpha-Q, h_{s}\right\rangle=\sum_{s=1}^{N_{f}}\left(\frac{b \varkappa}{N_{f}}+b\left\langle\lambda h_{1}-Q, h_{p_{s}}\right\rangle-n_{s}\right)=b \varkappa+k_{1} b \lambda+k_{2} b^{2}+k_{3}
$$

for some integers $k_{i}$ : this cannot happen for generic $(b, \varkappa, \lambda)$. Thus there exists $1 \leq u \leq N_{f}$ such that none of the $\phi_{p u}$ are integers. The condition (A.55) for $l=u$ then implies that for each $3 \leq k \leq N_{f}, \phi_{(k-1) t_{k}} \in \mathbb{Z}_{\geq 0}$ for some $1 \leq t_{k} \leq N_{f}$. No two $t_{k}$ can be equal, because $\phi_{(k-1) t}-\phi_{(l-1) t}=(k-l)\left(b^{2}+1\right)$ is non-integer for $k \neq l$. We can thus permute the components of $\alpha-Q$ through a Weyl transformation so that $t_{k}=k-1$ :

$$
b\left\langle\alpha-Q, h_{k-1}\right\rangle=b\left\langle-\varkappa h_{N_{f}}, h_{1}\right\rangle+b\left\langle\lambda h_{1}-Q, h_{k-1}\right\rangle-n_{k}
$$

for all $3 \leq k \leq N_{f}$, where $n_{k} \geq 0$ are some integers. We deduce that

$$
\alpha=(\lambda+\nu) h_{1}-(\varkappa+\nu) h_{N_{f}}+\frac{1}{b} \sum_{k=3}^{N_{f}} n_{k}\left(h_{1}-h_{k-1}\right) .
$$

The same considerations applied to the braiding of $-\frac{1}{b} h_{1}$ and $-\varkappa h_{N_{f}}$ yield the constraint above with $\frac{1}{b}$ replaced by $b$. We can thus restrict to momenta (A.58) which also have, up to a Weyl transformation, the $b \rightarrow \frac{1}{b}$ form. All in all, the fusion of two semi-degenerate operators can only allow a one-parameter set of momenta, and some isolated momenta

$$
\begin{aligned}
V_{-\varkappa h_{N_{f}}} \times V_{\lambda h_{1}}= & \int \mathrm{d} \nu V_{(\lambda+\nu) h_{1}-(\varkappa+\nu) h_{N_{f}}} \\
& +\sum_{n \in \mathbb{Z}} \sum_{n^{\prime} \in \mathbb{Z}} \sum_{k=1}^{N_{f}} V_{(\lambda-\varkappa) h_{1}+(n / b)\left(h_{1}-h_{k}\right)+\left[n^{\prime} b-\left(N_{f}-k\right) / b\right]\left(h_{1}-h_{N_{f}}\right)} .
\end{aligned}
$$

In the case $N_{f}=3$, we wrote down explicitly null vectors descending from $V_{-\varkappa h_{N_{f}}}$ and $V_{\lambda h_{1}}$ (higher $W_{N_{f}}$ algebras are intractable), and found that the isolated momenta are in fact not 
allowed. We propose that this holds for general $N_{f}$. After performing some Weyl reflections of momenta on the left and right-hand side and redefining $\nu$, we deduce the fusion rules

$$
\begin{aligned}
V_{-\varkappa h_{N_{f}}} \times V_{\lambda h_{1}} & =\int \mathrm{d} \nu V_{-\varkappa h_{N_{f}}+\lambda h_{1}+\nu\left(h_{1}-h_{N_{f}}\right)} \\
V_{\varkappa h_{1}} \times V_{\lambda h_{1}} & =\int \mathrm{d} \nu V_{\varkappa h_{1}+\lambda h_{1}+\nu\left(h_{1}-h_{2}\right)} \\
V_{-\varkappa h_{N_{f}}} \times V_{-\lambda h_{N_{f}}} & =\int \mathrm{d} \nu V_{-\varkappa h_{N_{f}}-\lambda h_{N_{f}}+\nu\left(h_{N_{f}-1}-h_{N_{f}}\right) .}
\end{aligned}
$$

For completeness, we find the corresponding structure constant as the main residue of $C\left(\alpha_{1}, \alpha_{2}, \varkappa h_{1}\right)$ at $\alpha_{1}=\lambda h_{1}$ and $\alpha_{2}=2 Q-(\varkappa+\lambda+\nu) h_{1}+\nu h_{2}$, after removing our normalization from (A.13), and recognize a Liouville CFT three-point function:

$$
\begin{aligned}
C_{\varkappa h_{1}, \lambda h_{1}}^{\left(\varkappa+\lambda+\nu h_{1}-\nu h_{2}\right.} & =\frac{\hat{\mu}^{\nu} \Upsilon(b)^{N_{f}-1} \Upsilon(\varkappa) \Upsilon(\lambda) \Upsilon(\varkappa+\lambda+2 \nu-b-1 / b)}{\Upsilon(-\nu) \Upsilon(\varkappa+\nu) \Upsilon(\lambda+\nu) \Upsilon(\varkappa+\lambda+\nu-b-1 / b)} \\
& =\Upsilon(b)^{N_{f}-2} C_{\text {Liouville }}\left(\frac{\varkappa}{2}, \frac{\lambda}{2}, b+\frac{1}{b}-\frac{\varkappa+\lambda}{2}-\nu\right) .
\end{aligned}
$$

The equality is true by construction for $N_{f}=2$, as a Liouville momentum of $\varkappa / 2$ corresponds in the Toda CFT language to a momentum of $(\varkappa / 2)\left(h_{1}-h_{2}\right)=\varkappa h_{1}$. More generally, the equality may hint to a deeper relation between Toda CFTs for different values of $N_{f}$.

\section{A.4.2 Fusion of semi-degenerate and degenerate operators}

We are now ready to tackle the fusion of other degenerate vertex operators $V_{-b \omega}$ with semi-degenerate operators $V_{\varkappa h_{1}}$.

For $\omega=N h_{1}$ the fusion is a special case of (A.61) with $\lambda=-N b$, hence only allows the momenta $(\varkappa-N b) h_{1}+\nu\left(h_{1}-h_{2}\right)$. Given the fusion rule (A.50) of a degenerate operator, $(N-\nu / b) h_{1}+(\nu / b) h_{2}$ must be a weight of $\mathcal{R}\left(N h_{1}\right)$ hence $\nu=n b$ with $0 \leq n \leq N$, and

$$
V_{\varkappa h_{1}} \times V_{-N b h_{1}}=\sum_{n=0}^{N} V_{(\varkappa-(N-n) b) h_{1}-n b h_{2}}
$$

with no multiplicity since the weight $(N-n) h_{1}+n h_{2}$ of $\mathcal{R}\left(N h_{1}\right)$ has no multiplicity. Through the Weyl rotation $\left(N_{f} \cdots 21\right)$, an equivalent statement is that the fusion of $-\varkappa h_{N_{f}}$ and $-N b h_{1}$ yields the momenta $-n b h_{1}-(\varkappa+(N-n) b) h_{N_{f}}$.

The correlator $\left\langle V_{\alpha_{\infty}}(\infty) V_{\left(\varkappa^{\prime}+l b\right) h_{1}}(1) V_{-l b h_{1}}(x, \bar{x}) V_{\alpha_{0}}(0)\right\rangle$ has $\operatorname{dim}\left(\mathcal{R}\left(l h_{1}\right)\right)$ s-channel conformal blocks, and must have the same number of t-channel conformal blocks. The fusion (A.64) allows the t-channel internal momenta $\varkappa^{\prime} h_{1}+n b\left(h_{1}-h_{2}\right)$ for $0 \leq n \leq l$, with no multiplicity, hence any multiplicity is due to the fusion of $V_{\alpha_{0}}$ and $V_{\alpha_{\infty}}$. The number of t-channel conformal blocks is thus

$$
\sum_{n=0}^{l} N_{\alpha_{0}, \alpha_{\infty}}^{\varkappa^{\prime} h_{1}+n b\left(h_{1}-h_{2}\right)}=\operatorname{dim} \mathcal{R}\left(l h_{1}\right)=\left(\begin{array}{c}
N_{f}+l-1 \\
l
\end{array}\right)
$$


where $V_{\beta}$ appears $N_{\alpha_{0}, \alpha_{\infty}}^{\beta}$ times in the fusion of $V_{\alpha_{0}}$ and $V_{\alpha_{\infty}}$. Solving, we find

$$
\begin{aligned}
N_{\alpha_{0}, \alpha_{\infty}}^{\varkappa^{\prime} h_{1}+n b\left(h_{1}-h_{2}\right)} & =\operatorname{dim} \mathcal{R}\left(n h_{1}\right)-\operatorname{dim} \mathcal{R}\left((n-1) h_{1}\right) \\
& =\left(\begin{array}{c}
N_{f}+n-1 \\
n
\end{array}\right)-\left(\begin{array}{c}
N_{f}+n-2 \\
n-1
\end{array}\right)=\left(\begin{array}{c}
N_{f}+n-2 \\
n
\end{array}\right) .
\end{aligned}
$$

None of these multiplicities vanish, so all $N+1$ momenta of (A.64) do appear in the fusion.

Restricting the fusion rule (A.64) to $\varkappa h_{1}=-K b h_{1}$ with $K \geq N$, we retrieve the decomposition into irreducible representations of the tensor product of two symmetric representations, given by the Littlewood-Richardson rule:

$$
\mathcal{R}\left(K h_{1}\right) \otimes \mathcal{R}\left(N h_{1}\right)=\bigoplus_{n=0}^{N} \mathcal{R}\left((K+N-n) h_{1}+n h_{2}\right) .
$$

One could go in the other direction: the decomposition (A.67) for $K \geq N$ implies that the fusion of $V_{-K b h_{1}}$ with $V_{-N b h_{1}}$ yields the momenta $-(K+N-n) b h_{1}-n b h_{2}$. This set of $N+1$ momenta only involves $-K b h_{1}$ as an overall constant part, hence the natural generalization from $V_{-K b h_{1}}$ to $V_{\varkappa h_{1}}$ is (A.64). We will apply a similar reasoning ${ }^{36}$ to guess the fusion of other degenerate operators with a semi-degenerate operator.

The tensor product of an antisymmetric and a symmetric representations of $A_{N_{f}-1}$ is the sum of two irreducible representations,

$$
\mathcal{R}\left(K h_{1}\right) \otimes \mathcal{R}\left(\omega_{N}\right)=\mathcal{R}\left(K h_{1}+\omega_{N}\right) \oplus \mathcal{R}\left((K-1) h_{1}+\omega_{N+1}\right) .
$$

This naturally generalizes to the fusion rule

$$
V_{\varkappa h_{1}} \times V_{-b \omega_{N}}=V_{\varkappa h_{1}-b \omega_{N}}+V_{\varkappa h_{1}-b\left(\omega_{N+1}-h_{1}\right)} .
$$

For completeness, a Weyl reflection yields the fusion of $-\varkappa h_{N_{f}}$ and $-b \omega_{N}$, which features the momenta $-(\varkappa+b) h_{N_{f}}-b \omega_{N-1}$ and $-\varkappa h_{N_{f}}-b \omega_{N}$.

We show in section 2.2, together with appendices A.3 and A.2 which do not depend on this fusion rule, that the Toda CFT correlator of two generic operators with $V_{\varkappa h_{1}}$ and $V_{-b \omega_{N}}$ is equal to the partition function of a surface operator. At the end of section 2.2.1 we expand the partition function in a limit which corresponds to the fusion of $V_{\varkappa h_{1}}$ and $V_{-b \omega_{N}}$. The exponents found there prove the fusion rule (A.69). Once more, the number of t-channel and s-channel conformal blocks must be equal:

$$
N_{\alpha_{0}, \alpha_{\infty}}^{\varkappa h_{1}-b \omega_{N}}+N_{\alpha_{0}, \alpha_{\infty}}^{(\varkappa+b) h_{1}-b \omega_{N+1}}=\left(\begin{array}{c}
N_{f} \\
N
\end{array}\right)
$$

We deduce for each $n \geq 0$ that for all $\varkappa$,

$$
N_{\alpha_{0}, \alpha_{\infty}}^{\varkappa h_{1}-b \omega_{n+1}}=\left(\begin{array}{c}
N_{f}-1 \\
n
\end{array}\right)
$$

\footnotetext{
${ }^{36}$ In principle, one could go further, and guess the fusion rule (A.61) for two semi-degenerate operators by replacing $-N b h_{1} \rightarrow \lambda h_{1}$ and allowing shifts by continuous multiples of $h_{2}-h_{1}$. It could be interesting to obtain a continuous analogue of the Littlewood-Richardson rule along those lines.
} 
This is consistent with multiplicities of the two powers of $|1-x|^{2}$ found at the end of section 2.2.1, and matches with (A.66) for $n=1$ and $n=0$.

Consider now an arbitrary highest weight $\omega=\sum_{j=1}^{N_{f}-1} n_{j} \omega_{j}$ of $A_{N_{f}-1}$. For each $j$ from $N_{f}-1$ to 1 , its Young diagram has $n_{j}$ columns with $j$ boxes. Through the LittlewoodRichardson rule, we find a decomposition valid for $K \geq \sum_{j=1}^{N_{f}-1} n_{j}$,

$$
\mathcal{R}\left(K h_{1}\right) \otimes \mathcal{R}(\omega)=\bigoplus_{k_{N_{f}-1}=0}^{n_{N_{f}-1}} \cdots \bigoplus_{k_{1}=0}^{n_{1}} \mathcal{R}\left(K h_{1}+\omega+\sum_{j=1}^{N_{f}-1}\left[k_{j}\left(h_{j+1}-h_{1}\right)\right]\right)
$$

into $\prod_{j=1}^{N_{f}-1}\left(n_{j}+1\right)$ irreducible representations. We thus propose the fusion rule

$$
V_{\varkappa h_{1}} \times V_{-b \sum_{j=1}^{N_{f}-1} n_{j} \omega_{j}}=\sum_{k_{1}=0}^{n_{1}} \cdots \sum_{k_{N_{f}-1}=0}^{n_{N_{f}-1}} V_{\varkappa h_{1}-b \sum_{j=1}^{N_{f}-1}\left[n_{j} \omega_{j}+k_{j}\left(h_{j+1}-h_{1}\right)\right]} .
$$

As a natural generalization of (A.66) and (A.71), we propose that vertex operators with a momentum $\varkappa h_{1}-b \sum_{j=1}^{N_{f}-1} l_{j} \omega_{j}$ appear with multiplicity

$$
N_{\alpha_{0}, \alpha_{\infty}}^{\varkappa h_{1}-b \sum_{j=1}^{N_{f}-1} l_{j} \omega_{j}}=\operatorname{dim} \mathcal{R}_{A_{N_{f}-2}}\left(\sum_{j=1}^{N_{f}-1} l_{j} \omega_{j-1}\right)
$$

where the $j=1$ term can be absorbed in a shift of $\varkappa$, and the right-hand side is the dimension of the representation of $A_{N_{f}-2}$ whose Young diagram is obtained from that of $\mathcal{R}\left(\sum_{j=1}^{N_{f}-1} l_{j} \omega_{j}\right)$ by removing the first row: $h_{1} \rightarrow 0$ and $h_{i} \rightarrow h_{i-1}$. Besides reproducing the correct multiplicities for the symmetric and antisymmetric case, the proposal (A.74) correctly leads to equally many s-channel and t-channel conformal blocks in the four-point function $\left\langle V_{\alpha_{\infty}}(\infty) V_{\varkappa h_{1}}(1) V_{-b \omega}(x, \bar{x}) V_{\alpha_{0}}(0)\right\rangle$ since

$$
\operatorname{dim} \mathcal{R}\left(\sum_{j=1}^{N_{f}-1} n_{j} \omega_{j}\right)=\sum_{k_{1}=0}^{n_{1}} \ldots \sum_{k_{N_{f}-1}=0}^{n_{N_{f}-1}} \operatorname{dim} \mathcal{R}_{A_{N_{f}-2}}\left(\sum_{j=1}^{N_{f}-1}\left[n_{j} \omega_{j-1}+k_{j} h_{j}\right]\right) .
$$

The equality holds because the representations on the right-hand side are the decomposition of $\mathcal{R}(\omega)$ into irreducible representations of the subalgebra $A_{N_{f}-2}$ of $A_{N_{f}-1}$.

\section{A.5 Conformal blocks}

In this section we give explicit expressions of conformal blocks which are labeled by two generic momenta $\alpha_{\infty}=Q-\mathrm{i} a_{\infty}$ and $\alpha_{0}=Q-\mathrm{i} a_{0}$ at $\infty$ and 0 , one semi-degenerate momentum $\hat{m}=\lambda h_{1}$ at 1 , and some degenerate momenta $-b \Omega_{j}$ inserted at the positions $\left(x_{j}, \bar{x}_{j}\right)$ for $1 \leq j \leq n$. The expressions are direct translations of the gauge theory vortex partition functions through the correspondence described in the main text. We only consider conformal blocks in the s-channel (the region $1>\left|x_{n}\right|>\cdots>\left|x_{1}\right|>0$ ), which are series in powers of $x_{n}, x_{n-1} / x_{n}, \ldots, x_{1} / x_{2}$. 
First comes the case of a single degenerate momentum $-b \omega_{N}$ labeled by the $N$-th antisymmetric representation of $A_{N_{f}-1}$. The four-point conformal blocks are equal, up to powers of $x$ and $1-x$, to the vortex partition functions (2.60) of SQCD,

$$
\begin{aligned}
& \mathcal{F}_{Q-\mathrm{i} a_{0}-b h_{\{p\}}}^{(\mathrm{s})}\left[\begin{array}{cc}
\lambda h_{1} & -b \omega_{N} \\
Q-\mathrm{i} a_{\infty} & Q-\mathrm{i} a_{0}
\end{array}\right](x) \\
& =x^{-b\left\langle\mathrm{i} a_{0}, h_{\{p\}}\right\rangle+\frac{N\left(N_{f}-N\right)}{2}\left(b^{2}+1\right)}(1-x)^{N\left(b^{2}+1-b \lambda / N_{f}\right)} \\
& \sum_{k_{1}, \ldots, k_{N} \geq 0} \frac{x^{\sum_{j=1}^{N} k_{j}}}{\prod_{j=1}^{N} k_{j} !} \frac{\prod_{j=1}^{N} \prod_{s=1}^{N_{f}}\left(1-b(\lambda-N b) / N_{f}-b\left\langle\mathrm{i} a_{0}, h_{p_{j}}\right\rangle-b\left\langle\mathrm{i} a_{\infty}, h_{s}\right\rangle\right)_{k_{j}}}{\prod_{i \neq j}^{N}\left(b\left\langle\mathrm{i} a_{0}, h_{p_{i}}-h_{p_{j}}\right\rangle-k_{i}\right)_{k_{j}} \prod_{j=1}^{N} \prod_{s \notin\{p\}}^{N_{f}}\left(1+b\left\langle\mathrm{i} a_{0}, h_{s}-h_{p_{j}}\right\rangle\right)_{k_{j}}} .
\end{aligned}
$$

The internal momentum $Q-\mathrm{i} a_{0}-b h_{\{p\}}$ is labeled by a weight $h_{\{p\}}=h_{p_{1}}+\cdots+h_{p_{N}}$ of $\mathcal{R}\left(\omega_{N}\right)$, where $1 \leq p_{1}<\cdots<p_{N} \leq N_{f}$. While the expression (A.76) is established since we provide a proof of the correspondence in this case, the conformal blocks below are not. However, they are supported by the evidence we gave for the correspondence in the main text.

Next, s-channel conformal blocks with the degenerate momentum $-N b h_{1}$ have an internal momentum $Q-\mathrm{i} a_{0}-b h$ labeled by a weight $h$ of the $N$-th symmetric representation $\mathcal{R}\left(N h_{1}\right)$ of $A_{N_{f}-1}$. We let $h=h_{[n]}=\sum_{s=1}^{N_{f}} n_{s} h_{s}$ for $\sum_{s=1}^{N_{f}} n_{s}=N$, and $I=\{(s, \mu) \mid 1 \leq$ $\left.s \leq N_{f}, 0 \leq \mu \leq n_{s}\right\}$. Conformal blocks are vortex partition functions (2.97) up to factors $x^{-\gamma_{0}}(1-x)^{-\gamma_{1}}$ from the correspondence (2.88):

$$
\begin{aligned}
\mathcal{F}_{Q-\mathrm{i} a_{0}-b h_{[n]}}^{(\mathrm{s})}\left[\begin{array}{cc}
\lambda h_{1} & -N b h_{1} \\
Q-\mathrm{i} a_{\infty} & Q-\mathrm{i} a_{0}
\end{array}\right](x) \\
=x^{\Delta\left(Q-\mathrm{i} a_{0}-b h_{[n]}\right)-\Delta\left(Q-\mathrm{i} a_{0}\right)-\Delta\left(-N b h_{1}\right)}(1-x)^{\Delta\left(\lambda h_{1}-N b h_{2}\right)-\Delta\left(\lambda h_{1}\right)-\Delta\left(-N b h_{1}\right)} \\
\sum_{k: I \rightarrow \mathbb{Z}_{\geq 0}} \prod_{(s, \mu) \in I}\left[x^{k_{s \mu}} \prod_{t=1}^{N_{f}} \frac{\left(1-b(\lambda-N b) / N_{f}-b\left\langle\mathrm{i} a_{0}, h_{s}\right\rangle-b\left\langle\mathrm{i} a_{\infty}, h_{t}\right\rangle-\mu b^{2}\right)_{k_{s \mu}}}{\left(1+b\left\langle\mathrm{i} a_{0}, h_{t}-h_{s}\right\rangle+\left(n_{t}-\mu\right) b^{2}\right)_{k_{s \mu}}}\right. \\
\left.\cdot \frac{\prod_{t=1}^{N_{f}}\left(1+b\left\langle\mathrm{i} a_{0}, h_{t}-h_{s}\right\rangle+\left(n_{t}-\mu\right) b^{2}+k_{s \mu}-k_{t\left(n_{t}-1\right)}\right)_{k_{t\left(n_{t}-1\right)}}}{\prod_{(t, \nu) \in I}\left(1+b\left\langle\mathrm{i} a_{0}, h_{t}-h_{s}\right\rangle+(\nu-\mu) b^{2}+k_{s \mu}-k_{t \nu}\right)_{k_{t \nu}-k_{t(\nu-1)}}}\right] .
\end{aligned}
$$

We now come to the case of $(n+3)$-point conformal blocks with two generic, one semi-degenerate, and $n$ degenerate momenta $-b \Omega_{j}=-b \Omega\left(K_{j}, \epsilon_{j}\right)$, where $\Omega(K,-1)=\omega_{K}$ and $\Omega(K,+1)=K h_{1}$. In the s-channel $1>\left|x_{n}\right|>\ldots>\left|x_{1}\right|$, the internal momentum running between the punctures at $x_{j}$ and $x_{j+1}$ (here $x_{n+1}=1$ ) has the form $\alpha_{0}-b h_{\left[n^{j}\right]}=$ $\alpha_{0}-b \sum_{t=1}^{N_{f}} n_{t}^{j} h_{t}$, for some integers $n_{t}^{j} \geq 0$. These integers must be such that $h_{\left[n^{j}\right]}-h_{\left[n^{j-1}\right]}$ is a weight of $\mathcal{R}\left(\Omega_{j}\right)$ for each $1 \leq j \leq n$ (here $n_{t}^{0}=0$ ). Explicitly, $\sum_{t=1}^{N_{f}}\left(n_{t}^{j}-n_{t}^{j-1}\right)=K_{j}$, and $n_{t}^{j}-n_{t}^{j-1}$ is in $\mathbb{Z}_{\geq 0}$ if $\epsilon_{j}=+1$ and in $\{0,1\}$ if $\epsilon_{j}=-1$.

In section 2.4 we find a quiver gauge theory whose vacua are labeled by the same data, and perform various checks that its partition function is equal to the Toda correlator we are now considering. Up to simple factors, the conformal blocks are thus equal to the vortex partition functions, themselves a sum of residues in the Coulomb branch representation of 
the partition function. Let us introduce the sets $I_{j}=\left\{(s, \mu) \mid 0 \leq \nu<n_{s}^{j}, 1 \leq s \leq N_{f}\right\}$ ( $I_{0}$ is empty), the notation $\mathrm{i} a_{s, \mu}=\left\langle\mathrm{i} a_{0}, h_{s}\right\rangle+\mu b$, and the parameters $q_{n+1}=b^{2} / 2$ and $q_{j}=\epsilon_{j}\left(b^{2} / 2+1 / 4\right)-1 / 4$. We find

$$
\begin{aligned}
& \mathcal{F}^{(\mathrm{s})}\left[\begin{array}{cccc}
\lambda h_{1} & -b \Omega_{n} & -b \Omega_{2} & -b \Omega_{1} \\
\alpha_{\infty}, \downarrow \alpha_{0}-b h_{\left[n^{n}\right]} \downarrow & \cdots & \downarrow \alpha_{0}-b h_{\left[n^{1}\right]} \downarrow & \alpha_{0}
\end{array}\right](x) \\
& =\prod_{j=1}^{n}\left[x_{j}^{\Delta\left(\alpha_{0}-b h_{[n j]}\right)-\Delta\left(\alpha_{0}-b h_{[n j-1]}\right)-\Delta\left(-b \Omega_{j}\right)}\left[1-x_{j}\right]^{\left(1+b^{2}-\frac{b \lambda}{N_{f}}\right) K_{j}}\right] \prod_{i<j}^{n}\left[1-\frac{x_{i}}{x_{j}}\right]^{\left(1+2 q_{j}+b^{2} \frac{K_{j}}{N_{f}}\right) K_{i}} \\
& \cdot \sum_{\left\{k_{j, s, \mu} \geq 0\right\}}\left\{\prod _ { j = 1 } ^ { n } \prod _ { ( s , \mu ) \in I _ { j } } \left[\left[\frac{x_{j}}{x_{j+1}}\right]^{k_{j, s, \mu}} \prod_{(t, \nu) \in I_{j}} \frac{\left(1+b \mathrm{i} a_{s, \mu}-b \mathrm{i} a_{t, \nu}\right)_{k_{j, t, \nu}-k_{j, s, \mu}}}{\left(1+q_{j}+q_{j+1}+b \mathrm{i} a_{s, \mu}-b \mathrm{i} a_{t, \nu}\right)_{k_{j, t, \nu}-k_{j, s, \mu}}}\right.\right. \\
& \left.\cdot \prod_{(t, \nu) \in I_{j-1}} \frac{\left(1+2 q_{j}+b \mathrm{i} a_{s, \mu}-b \mathrm{i} a_{t, \nu}\right)_{k_{j-1, t, \nu}-k_{j, s, \mu}}}{\left(1+b \mathrm{i} a_{s, \mu}-b \mathrm{i} a_{t, \nu}\right)_{k_{j-1, t, \nu}-k_{j, s, \mu}}}\right] \\
& \left.\cdot \prod_{s=1}^{N_{f}} \prod_{(t, \nu) \in I_{n}} \frac{\left(1-b\left(\lambda-b \sum_{j=1}^{n} K_{j}\right) / N_{f}-b\left\langle\mathrm{i} a_{\infty}, h_{s}\right\rangle-b \mathrm{i} a_{t, \nu}\right)_{k_{n, t, \nu}}}{\left(1+b\left\langle\mathrm{i} a_{0}, h_{s}\right\rangle-b \mathrm{i} a_{t, \nu}\right)_{k_{n, t, \nu}}}\right\} \text {. }
\end{aligned}
$$

As discussed in the main text, when all $\epsilon_{j}=-1$, placing all degenerate punctures at the same position $x_{j}=x$ yields the conformal block for one particular fusion of the degenerate momenta, which turns out to be

$$
-b \Omega=-b \sum_{j=1}^{n} \Omega_{j}=-b \sum_{j=1}^{n} \omega_{K_{j}} .
$$

This provides an explicit expression for the four-point conformal block of two generic and one semi-degenerate momentum, and one degenerate momentum labeled by an arbitrary representation of $A_{N_{f}-1}$. Fusing degenerate punctures in several sets gives conformal blocks with several arbitrary degenerate momenta $-b \Omega$, but these quickly become unwieldy.

\section{A.6 Irregular punctures}

We study irregular punctures obtained as collision limits of vertex operators in the Toda CFT. Such collisions were studied for Virasoro primaries in [51], and extended to other algebras in $[60,61]$. We give evidence that the limit

$$
\mathbb{V}_{c_{0} ; c_{1}, \bar{c}_{1} ; \cdots ; c_{K}, \bar{c}_{K}}(w, \bar{w})=\lim _{\left(w_{I}, \bar{w}_{I}\right) \rightarrow(w, \bar{w})} \prod_{I<J}\left|w_{J}-w_{I}\right|^{2\left\langle\alpha_{J}, \alpha_{I}\right\rangle} \prod_{I=0}^{K} V_{\alpha_{I}}\left(w_{I}, \bar{w}_{I}\right)
$$

exists, provided that the momenta $\alpha_{I}$ of vertex operators, and their position $\left(w_{J}, \bar{w}_{J}\right)$, vary in such a way that

$$
C_{j}=\sum_{I=0}^{K}\left(w_{I}-w\right)^{j} \alpha_{I} \rightarrow c_{j} \quad \bar{C}_{j}=\sum_{I=0}^{K}\left(\bar{w}_{I}-\bar{w}\right)^{j} \alpha_{I} \rightarrow \bar{c}_{j}
$$


for all $j \geq 0$. Not every choice of $c_{j}$ and $\bar{c}_{j}$ can appear (for a given rank $K$ ). Firstly, $\bar{c}_{0}=c_{0}$. Secondly, $\bar{c}_{j}=c_{j}=0$ for all $j>K$. Indeed, any $C_{j}$ with $j>K$ is a linear combination $C_{j}=\sum_{k=0}^{K} P_{j, k}\left(\left\{w_{I}-w\right\}\right) C_{k}$ whose coefficients $P_{j, k}$ are homogeneous polynomial of degree $j-k \geq 1$ in the variables $w_{I}-w$, and such polynomials vanish as $w_{I} \rightarrow w$. The limits of $C_{j}$ and $\bar{C}_{j}$ are thus described by the $2 K+1$ momenta $\left(c_{0} ; c_{1}, \bar{c}_{1} ; \cdots ; c_{K}, \bar{c}_{K}\right)$, as indicated by the notation in (A.80).

There is (at least) one other condition on the $c_{j}$ and $\bar{c}_{j}$ : for each $0 \leq m \leq K$ the vectors $\left\{c_{n}, \bar{c}_{n} \mid m \leq n \leq K\right\}$ must span a space of dimension at most $K-m+1$, for instance $c_{K}$ and $\bar{c}_{K}$ must be collinear. This third restriction relies on

$$
\sum_{j=0}^{n}\left(C_{j} \sum_{\substack{S \in \llbracket 0, n-1 \rrbracket \\ \# S=n-j}} \prod_{I \in S}\left(w-w_{I}\right)\right)=\sum_{J=n}^{K} \prod_{I=0}^{n-1}\left(w_{J}-w_{I}\right)^{n} \alpha_{J}
$$

whose left-hand side goes to $c_{n}$ in our limit, and on its analogue for $\bar{c}_{n}$. Since rank is lower semicontinuous, the rank of the space spanned by $\left\{c_{n}, \bar{c}_{n} \mid m \leq n \leq K\right\}$ is at most that of the space spanned by (A.82) and by their antiholomorphic counterparts (for $m \leq n \leq K$ ). This second space lies within the span of $\left\{\alpha_{J} \mid m \leq J \leq K\right\}$, which has rank at most $K-m+1$.

\section{A.6.1 OPE with the stress-energy tensor}

Our first piece of evidence is to write the OPE of the stress-energy tensor with

$$
\mathbf{V}_{\left\{\alpha_{I}\right\}}\left(\left\{w_{I}, \bar{w}_{I}\right\}\right)=\prod_{I<J}\left|w_{J}-w_{I}\right|^{2\left\langle\alpha_{J}, \alpha_{I}\right\rangle} \prod_{I=0}^{K} V_{\alpha_{I}}\left(w_{I}, \bar{w}_{I}\right)
$$

in the limit which defines $\mathbb{V}_{c_{0} ; \cdots ; c_{K}, \bar{c}_{K}}$. The operators $V_{\alpha_{I}}$ are primary, hence

$$
\begin{aligned}
& T(z) \mathbf{V}_{\left\{\alpha_{I}\right\}}\left(\left\{w_{I}, \bar{w}_{I}\right\}\right) \\
& \sim \prod_{I<J}\left|w_{J}-w_{I}\right|^{2\left\langle\alpha_{J}, \alpha_{I}\right\rangle} \sum_{I=0}^{K}\left(\frac{\Delta\left(\alpha_{I}\right)}{\left(z-w_{I}\right)^{2}}+\frac{1}{z-w_{I}} \partial_{w_{I}}\right) \prod_{I=0}^{K} V_{\alpha_{I}}\left(w_{I}, \bar{w}_{I}\right) \\
&=\sum_{I=0}^{K}\left(\frac{\Delta\left(\alpha_{I}\right)}{\left(z-w_{I}\right)^{2}}+\frac{1}{z-w_{I}}\left(\partial_{w_{I}}+\sum_{J \neq I} \frac{\left\langle\alpha_{I}, \alpha_{J}\right\rangle}{w_{J}-w_{I}}\right)\right) \mathbf{V}_{\left\{\alpha_{I}\right\}}\left(\left\{w_{I}, \bar{w}_{I}\right\}\right) \\
&=\left(\left\langle Q, \partial_{z} \partial_{z} \varphi_{\text {sing }}\right\rangle-\frac{1}{2}\left\langle\partial_{z} \varphi_{\text {sing }}, \partial_{z} \varphi_{\text {sing }}\right\rangle+\sum_{I=0}^{K} \frac{\partial_{w_{I}}}{z-w_{I}}\right) \mathbf{V}_{\left\{\alpha_{I}\right\}}\left(\left\{w_{I}, \bar{w}_{I}\right\}\right)
\end{aligned}
$$

where in the last line we use $\Delta\left(\alpha_{I}\right)=\left\langle Q, \alpha_{I}\right\rangle-\frac{1}{2}\left\langle\alpha_{I}, \alpha_{I}\right\rangle$ to express all but the $\partial_{w_{I}}$ piece in terms of

$$
\partial_{z} \varphi_{\text {sing }}=\sum_{I=0}^{K} \frac{-\alpha_{I}}{z-w_{I}}=\sum_{n \geq 0} \frac{-\sum_{I=0}^{K}\left(w_{I}-w\right)^{n} \alpha_{I}}{(z-w)^{n+1}}=\sum_{n \geq 0} \frac{-C_{n}}{(z-w)^{n+1}} \rightarrow \sum_{n=0}^{K} \frac{-c_{n}}{(z-w)^{n+1}} .
$$


In the domain where all $\left|w_{I}-w\right|<|z-w|$, we can expand the derivative term as

$$
\sum_{I=0}^{K}\left(z-w_{I}\right)^{-1} \partial_{w_{I}}=\sum_{n \geq-1}(z-w)^{-n-2} \sum_{I=0}^{K}\left(w_{I}-w\right)^{n+1} \partial_{w_{I}} .
$$

The term with $n=-1$ is $\sum_{I=0}^{K} \partial_{w_{I}}$, which translates all vertex operators, hence its limit is $\partial_{w}$. The other terms do not have such a simple geometrical interpretation. Instead, let us write their action on $C_{m}$ for $0 \leq m \leq K$ :

$$
\begin{aligned}
\sum_{I=0}^{K}\left(w_{I}-w\right)^{n+1} \partial_{w_{I}} C_{m} & =\sum_{I=0}^{K}\left(w_{I}-w\right)^{n+1} \partial_{w_{I}} \sum_{J=0}^{K}\left(w_{J}-w\right)^{m} \alpha_{J} \\
& =\sum_{I=0}^{K} m\left(w_{I}-w\right)^{n+m} \alpha_{I}=m C_{n+m} .
\end{aligned}
$$

The limit of $\sum_{I=0}^{K}\left(w_{I}-w\right)^{n+1} \partial_{w_{I}}$ must thus be a differential operator which maps $c_{m} \rightarrow$ $m c_{n+m}$ for all $0 \leq m \leq K-n$ and $c_{m} \rightarrow 0$ for $K-n<m \leq K$. This is naturally realized by

$$
\sum_{I=0}^{K}\left(w_{I}-w\right)^{n+1} \partial_{w_{I}} \rightarrow \sum_{j=1}^{K-n} j\left\langle c_{n+j}, \partial_{c_{j}}\right\rangle
$$

All in all, the OPE of $T(z)$ with $\mathbb{V}=\mathbb{V}_{c_{0} ; \cdots ; c_{K}, \bar{c}_{K}}(w, \bar{w})$ is

$$
\begin{aligned}
T(z) \mathbb{V} \sim & \left(\left\langle Q, \partial_{z} \sum_{n=0}^{K} \frac{-c_{n}}{(z-w)^{n+1}}\right\rangle-\frac{1}{2}\left\langle\sum_{j=0}^{K} \frac{-c_{j}}{(z-w)^{j+1}}, \sum_{l=0}^{K} \frac{-c_{l}}{(z-w)^{l+1}}\right\rangle\right. \\
& \left.+\frac{\partial_{w}}{z-w}+\sum_{n \geq 0} \frac{1}{(z-w)^{n+2}} \sum_{j=1}^{K-n} j\left\langle c_{n+j}, \partial_{c_{j}}\right\rangle\right) \mathbb{V} \\
= & \left(\frac{1}{z-w} \partial_{w}+\sum_{n=0}^{2 K} \frac{(n+1)\left\langle Q, c_{n}\right\rangle-\frac{1}{2} \sum_{j=0}^{n}\left\langle c_{j}, c_{n-j}\right\rangle+\sum_{j=1}^{K-n} j\left\langle c_{n+j}, \partial_{c_{j}}\right\rangle}{(z-w)^{n+2}}\right) \mathbb{V}
\end{aligned}
$$

where we recall that $c_{n}=0$ for $n>K$. The presence of singularities up to $(z-w)^{-2 K-2}$ in this OPE implies that the Virasoro generators $L_{n}$ act non-trivially on the state $|c\rangle=$ $\mathbb{V}_{c_{0} ; \cdots ; c_{K}, \bar{c}_{K}}(0)|0\rangle$ for $n \leq 2 K$. More precisely,

$$
L_{n}|c\rangle=\left((n+1)\left\langle Q, c_{n}\right\rangle-\frac{1}{2} \sum_{j=0}^{n}\left\langle c_{j}, c_{n-j}\right\rangle+\sum_{j=1}^{K-n} j\left\langle c_{n+j}, \partial_{c_{j}}\right\rangle\right)|c\rangle
$$

for $0 \leq n \leq 2 K$, while $L_{-1}$ translates $w$, and $L_{n}|c\rangle=0$ for $n>2 K$. This is the natural generalization of equation (2.7) of [51].

In the rank 1 case $\left(c_{n}=0\right.$ for $\left.n>1\right)$, we can exponentiate explicitly the action of the Virasoro generators $L_{n}$ to find how large conformal transformations act. From above, we 
know that $L_{n}|c\rangle=0$ for $n>2$, that $L_{-1}$ acts like $\partial_{w}$, and that

$$
\begin{aligned}
L_{2}|c\rangle & =-\frac{1}{2}\left\langle c_{1}, c_{1}\right\rangle|c\rangle, \\
L_{1}|c\rangle & =\left\langle 2 Q-c_{0}, c_{1}\right\rangle|c\rangle, \\
L_{0}|c\rangle & =\left(\Delta\left(c_{0}\right)+\left\langle c_{1}, \partial_{c_{1}}\right\rangle\right)|c\rangle,
\end{aligned}
$$

where as usual $\Delta\left(c_{0}\right)=\left\langle Q, c_{0}\right\rangle-\left\langle c_{0}, c_{0}\right\rangle / 2$. Omitting the parameters $\bar{z}, \bar{w}$ and $\bar{c}_{n}$ which play no role for holomorphic transformations, we claim that

$$
\mathbb{V}_{c_{0}, c_{1}}(z)=\left(\partial_{z} w\right)^{\Delta\left(c_{0}\right)} \exp \left(\frac{\left\langle 2 Q-c_{0}, c_{1}\right\rangle}{2} \frac{\partial_{z}^{2} w}{\partial_{z} w}-\frac{\left\langle c_{1}, c_{1}\right\rangle}{12}\left[\frac{\partial_{z}^{3} w}{\partial_{z} w}-\frac{3}{2} \frac{\left(\partial_{z}^{2} w\right)^{2}}{\left(\partial_{z} w\right)^{2}}\right]\right) \mathbb{V}_{c_{0},\left(\partial_{z} w\right) c_{1}}(w)
$$

under a conformal map $z \rightarrow w(z)$. Indeed, this transformation is transitive and has the correct infinitesimal behavior: for $\partial_{z} w=1+\epsilon$,

$$
\mathbb{V}_{c_{0}, c_{1}}(z)=\left(1+\epsilon\left(\Delta\left(c_{0}\right)+\left\langle c_{1}, \partial_{c_{1}}\right\rangle\right)+\frac{\left\langle 2 Q-c_{0}, c_{1}\right\rangle}{2} \partial_{z} \epsilon-\frac{\left\langle c_{1}, c_{1}\right\rangle}{12} \partial_{z}^{2} \epsilon+O\left(\epsilon^{2}\right)\right) \mathbb{V}_{c_{0}, c_{1}}(w)
$$

\section{A.6.2 Free field realization}

Our derivation of (A.93) only relies on the OPE of $T(z)$ with vertex operators $V_{\alpha}$. This OPE has a free field realization as the OPE of $T_{Q}^{\text {free }}=\langle Q, \partial \partial \varphi\rangle-\frac{1}{2}:\langle\partial \varphi, \partial \varphi\rangle$ : with $V_{\alpha}^{\text {free }}=$ $: e^{\langle\alpha, \varphi\rangle}$ :. We rederive (A.93) more directly by first building the collision limit $\mathbb{V}^{\text {free }}$ of vertex operators $V_{\alpha}^{\text {free }}$, then computing its OPE with $T_{Q}^{\text {free }}$. We then go further and consider the OPE of higher spin currents of the $W_{N_{f}}$ algebra with $\mathbb{V}^{\text {free }}$.

First, : $e^{\langle\alpha, \varphi(z, \bar{z})\rangle}:: e^{\langle\beta, \varphi(w, \bar{w})\rangle}:=|z-w|^{-2\langle\alpha, \beta\rangle}: e^{\langle\alpha, \varphi(z, \bar{z})\rangle+\langle\beta, \varphi(w, \bar{w})\rangle}$ : implies by induction

$$
\prod_{I<J}\left|w_{I}-w_{J}\right|^{2\left\langle\alpha_{I}, \alpha_{J}\right\rangle} \prod_{I=0}^{K}: e^{\left\langle\alpha_{I}, \varphi\left(w_{I}, \bar{w}_{I}\right)\right\rangle}:=: e^{\sum_{I=0}^{K}\left\langle\alpha_{I}, \varphi\left(w_{I}, \bar{w}_{I}\right)\right\rangle}: .
$$

Expanding $\varphi\left(w_{I}, \bar{w}_{I}\right)=\varphi(w, \bar{w})+\sum_{n>1} \frac{1}{n !}\left(\left(w_{I}-w\right)^{n} \partial^{n} \varphi(w)+\left(\bar{w}_{I}-\bar{w}\right)^{n} \bar{\partial}^{n} \varphi(\bar{w})\right)$ thanks to $\partial \bar{\partial} \varphi=0$ and using the limit $\sum_{I=0}^{K}\left(w_{I}-w\right)^{n}\left\langle\alpha_{I}, \partial^{n} \varphi\right\rangle \rightarrow\left\langle c_{n}, \partial^{n} \varphi\right\rangle$ and its antiholomorphic counterpart yields the free field collision limit

$$
\mathbb{V}_{c_{0} ; \cdots ; c_{K}, \bar{c}_{K}}^{\mathrm{free}}(w, \bar{w})=: \exp \left(\left\langle c_{0}, \varphi(w, \bar{w})\right\rangle+\sum_{n=1}^{K} \frac{1}{n !}\left(\left\langle c_{n}, \partial^{n} \varphi(w)\right\rangle+\left\langle\bar{c}_{n}, \bar{\partial}^{n} \varphi(\bar{w})\right\rangle\right)\right): .
$$

The stress-energy tensor $T_{Q}^{\text {free }}(z)$ and higher spin currents are polynomials in $\partial \varphi(z)$ and its derivatives. We thus evaluate

$$
\begin{aligned}
\partial \varphi(z) \mathbb{V}_{c_{0} ; \cdots}^{\text {free }}(w, \bar{w}) & =:\left(\partial \varphi(z)+\sum_{n \geq 0} \frac{c_{n}}{n !} \partial_{w}^{n} \frac{-1}{z-w}\right) \mathbb{V}_{c_{0} ; \cdots}^{\text {free }}(w, \bar{w}): \\
& =:\left(\sum_{n \geq 1}(z-w)^{n-1} n \partial_{c_{n}}-\sum_{n \geq 0} \frac{c_{n}}{(z-w)^{n+1}}\right) \mathbb{V}_{c_{0} ; \cdots}^{\mathrm{free}}(w, \bar{w}):
\end{aligned}
$$


where the first equality relies on $\partial \overline{\varphi(z)} \varphi(w, \bar{w})=-1 /(z-w)$, and the second on the Taylor expansion of $\partial \varphi(z)$ and on $\partial^{n} \varphi \mathbb{V}_{c_{0} ; \cdots}^{\text {free }}=n ! \partial_{c_{n}} \mathbb{V}_{c_{0} ; \cdots}^{\text {free }}$. The OPE of $\mathbb{V}_{c_{0} ; \cdots}^{\text {free }}(w, \bar{w})$ with any polynomial in derivatives of $\partial \varphi(z)$ is thus obtained by replacing all

$$
\partial_{z}^{l+1} \varphi(z) \rightarrow \partial_{z}^{l}\left(\sum_{n \geq 1}(z-w)^{n-1} n \partial_{c_{n}}-\sum_{n \geq 0} \frac{c_{n}}{(z-w)^{n+1}}\right)=-\partial_{z}^{l} \sum_{n \in \mathbb{Z}} \frac{\theta_{n} c_{n}+\theta_{-n} n \partial_{c_{-n}}}{(z-w)^{n+1}}
$$

where $\theta_{n}=1$ if $n \geq 0$ and 0 if $n<0$, then dropping terms that are regular as $z \rightarrow w$.

In particular,

$$
\begin{aligned}
T_{Q}^{\mathrm{free}}(z) \mathbb{V}^{\mathrm{free}}= & \left(\langle Q, \partial \partial \varphi(z)\rangle-\frac{1}{2}:\langle\partial \varphi(z), \partial \varphi(z)\rangle:\right) \mathbb{V}_{c_{0} ; \cdots ; c_{K}, \bar{c}_{K}}^{\mathrm{free}}(w, \bar{w}) \\
\sim & \left(\left\langle Q, \sum_{n \geq 0} \frac{(n+1) c_{n}}{(z-w)^{n+2}}\right\rangle-\frac{1}{2}\left\langle\sum_{i \geq 0} \frac{c_{i}}{(z-w)^{i+1}}, \sum_{j \geq 0} \frac{c_{j}}{(z-w)^{j+1}}\right\rangle\right. \\
& \left.\quad+\left\langle\sum_{i \geq 0} \frac{c_{i}}{(z-w)^{i+1}}, \sum_{j \geq 1}(z-w)^{j-1} j \partial_{c_{j}}\right\rangle\right) \mathbb{V}^{\text {free }} \\
= & \left(\sum_{n=0}^{2 K} \frac{(n+1)\left\langle Q, c_{n}\right\rangle-\frac{1}{2} \sum_{i=0}^{n}\left\langle c_{i}, c_{n-i}\right\rangle}{(z-w)^{n+2}}+\sum_{n=-1}^{K-1} \frac{\sum_{j=1}^{K-n}\left\langle c_{j+n}, j \partial_{c_{j}}\right\rangle}{(z-w)^{n+2}}\right) \mathbb{V}^{\text {free }} .
\end{aligned}
$$

Upper bounds could be omitted since $c_{m}=0$ for $m \geq K$. Note the presence of $\partial_{c_{K+1}}$ in the last term for $n=-1$ and $j=K+1$. This derivative is inconvenient as it involves irregular punctures with a rank higher than $\mathbb{V}^{\text {free }}$. It turns out that the terms with $n=-1$ combine nicely into

$$
\sum_{j=1}^{K+1} \frac{\left\langle c_{j-1}, \partial^{j} \varphi(w)\right\rangle}{(j-1) !} \mathbb{V}_{c_{0} ; \cdots ; c_{K}, \bar{c}_{K}}^{\mathrm{free}}(w, \bar{w})=\partial_{w} \mathbb{V}_{c_{0} ; \cdots ; c_{K}, \bar{c}_{K}}^{\mathrm{free}}(w, \bar{w})
$$

As expected, the free field OPE reproduces the OPE (A.93).

We are ready to consider higher spin currents. A basis of those currents is obtained via the Miura transform

$$
\prod_{s=N_{f}}^{1}\left(q \partial_{z}+\left\langle h_{s}, \partial_{z} \varphi(z)\right\rangle\right)=\sum_{p=0}^{N_{f}} W^{p}(z)\left(q \partial_{z}\right)^{N_{f}-p}
$$

where $q=b+\frac{1}{b}$. In particular, $W^{0}(z)=1, W^{1}(z)=0$, and $W^{2}(z)=T_{Q}^{\text {free }}(z)$. The prescription (A.104) then yields the OPE of $W^{p}(z)$ with the irregular $\mathbb{V}_{c_{0} ; \cdots}^{\text {free }}(w, \bar{w})$, but expressions quickly become very unwieldy. However, we can get valuable information by applying the prescription (A.104) directly to the Miura transform (A.109):

$$
\sum_{p=0}^{N_{f}} W^{p}(z) \mathbb{V}_{c_{0} ; \cdots}^{\text {free }}(w, \bar{w})\left(q \partial_{z}\right)^{N_{f}-p}=\prod_{s=N_{f}}^{1}\left(q \partial_{z}+\sum_{n \in \mathbb{Z}} \frac{\left\langle h_{s},-\theta_{n} c_{n}-\theta_{-n} n \partial_{c_{-n}}\right\rangle}{(z-w)^{n+1}}\right) \mathbb{V}_{c_{0} ; \cdots}^{\text {free }}(w, \bar{w})
$$


where $\partial_{c_{j}}$ only acts on $\mathbb{V}^{\text {free }}$ and not on intervening $c_{j}$, and where $\theta_{n}=1$ if $n \geq 0$ and 0 otherwise. The sums over $n$ actually truncate to $n \leq K$ for rank $K$ punctures, thus only a finite number of negative powers of $(z-w)$ appear in the OPE.

Let us find out the most singular terms of the OPE of a given $W^{p}(z)$ with $\mathbb{V}^{\text {free }}$ as $z \rightarrow w$. Thanks to the mode expansion $W^{p}(z)=\sum_{n \in \mathbb{Z}} W_{n}^{p}(w)(z-w)^{-n-p}$, the $(z-w)^{-n-p}$ term in the OPE encodes the action of $W_{n}^{p}$ on the rank $K$ puncture

$|c\rangle=\mathbb{V}_{c_{0} ; \cdots ; c_{K}, \bar{c}_{K}}^{\mathrm{free}}(w, \bar{w})|0\rangle$. Terms where $0 \leq m<p$ of the $q \partial_{z}$ act on some $\left\langle h_{s}, \ldots\right\rangle$ are at most of order $O\left((z-w)^{-(K+1)(p-m)-m}\right)$. Those involving $\partial_{c_{j}}$ derivatives are of order $O\left((z-w)^{-(K+1)(p-m-1)-m}\right)$ or more regular. Thus, $W_{n}^{p}|c\rangle=0$ for $n>p K$,

$$
\begin{aligned}
W_{n}^{p}|c\rangle=(-1)^{p} \sum_{1 \leq s_{1}<\cdots<s_{p} \leq N_{f}} & {\left[\sum_{k_{1}+\cdots+k_{p}=p K-n} \prod_{i=1}^{p}\left\langle h_{s_{i}}, c_{K-k_{i}}\right\rangle\right.} \\
& \left.+\delta_{n,(p-1) K}(K+1) q \sum_{j=1}^{p}\left((j-1) \prod_{i \neq j}^{p}\left\langle h_{s_{i}}, c_{K}\right\rangle\right)\right]|c\rangle
\end{aligned}
$$

for $(p-1) K \leq n \leq p K$, and lower components of $W^{p}(z)$ act with $\partial_{c_{j}}$ derivatives. This is consistent with the action (A.94) of the Virasoro algebra for $p=2$.

For $n<(p-1) K$, the action of $W_{n}^{p}$ on $|c\rangle$ involves derivatives $\partial_{c_{j}}$ for each $1 \leq j \leq$ $(p-1) K-n$. In particular, if $n<(p-2) K$, derivatives with $j>K$ appear: the set of rank $K$ irregular punctures is not stable under those components $W_{n}^{p}$. One exception is that $L_{-1}=W_{-1}^{2}$ involves derivatives up to $\partial_{c_{K+1}}$ but turns out to be identical to an infinitesimal translation. The set of all (finite, integer) rank irregular punctures is stable under all $W_{n}^{p}$.

Before closing this appendix, we go back to the Toda CFT and compute various twopoint functions of vertex operators with rank $K=1$ irregular punctures as a test that the collision limit is finite.

\section{A.6.3 Two-point functions}

Irregular punctures only arise in section 2.2.3 as the collision of a semi-degenerate and a generic vertex operators. We compute here the two-point function of the resulting rank 1 puncture with any generic vertex operator (A.116) in a useful normalization (A.113).

The collision limits of interest are a special case of the general collision limit (A.80) which defines rank $K$ irregular punctures. Using notations close to the main text,

$$
\mathbb{V}_{c_{0} ;-(x / b) h_{1},(\bar{x} / b) h_{1}}(0)=\lim _{\Lambda \rightarrow \infty}\left[\left|\frac{x}{\Lambda}\right|^{2\left\langle\varkappa h_{1}, c_{0}-\varkappa h_{1}\right\rangle} V_{\varkappa h_{1}}\left(\frac{x}{-\mathrm{i} \Lambda}, \frac{\bar{x}}{\mathrm{i} \Lambda}\right) V_{c_{0}-\varkappa h_{1}}(0)\right]_{\varkappa=\mathrm{i} \Lambda / b+O(1)}
$$

where $\Lambda \in \mathbb{R}$ is the gauge theory cutoff scale, $c_{0}, b, x$ and $\bar{x}$ are various physical parameters, and only the leading behavior of $\varkappa$ in $\Lambda$ affects the limit. We also introduce the 
normalization

$$
\begin{aligned}
& \widehat{\mathbb{V}}_{c_{0} ;-(x / b) h_{1},(\bar{x} / b) h_{1}}(0)=\frac{\hat{\mu}^{\left\langle c_{0}-Q, \rho\right\rangle} \mathbb{V}_{c_{0} ;-(x / b) h_{1},(\bar{x} / b) h_{1}}(0)}{\Upsilon(b)^{N_{f}-1} \prod_{2 \leq s<t \leq N_{f}} \Upsilon\left(\left\langle Q-c_{0}, h_{s}-h_{t}\right\rangle\right)} \\
& \quad=\lim _{\Lambda \rightarrow \infty}\left[\frac{\Upsilon\left(\varkappa+\left\langle Q-c_{0}, h_{1}\right\rangle\right)^{N_{f}}}{|\Lambda / b|^{2 \Delta\left(c_{0}\right)-\langle Q, Q\rangle}}\left|\frac{x}{\Lambda}\right|^{2\left\langle\varkappa h_{1}, c_{0}-\varkappa h_{1}\right\rangle} \widehat{V}_{\varkappa h_{1}}\left(\frac{x}{-\mathrm{i} \Lambda}, \frac{\bar{x}}{\mathrm{i} \Lambda}\right) \widehat{V}_{c_{0}-\varkappa h_{1}}(0)\right]_{\varkappa=\mathrm{i} \Lambda / b+O(1)}
\end{aligned}
$$

where the second line is obtained by combining the factors (A.7) and (A.8) which relate $\widehat{V}$ and $V$ with those relating $\widehat{\mathbb{V}}$ and $\mathbb{V}$. The only non-trivial step is that the asymptotics (A.11) of the Upsilon function simplify $\prod_{t=1}^{N_{f}} \Upsilon\left(\varkappa+\left\langle Q-c_{0}, h_{1}-h_{t}\right\rangle\right)$ to $\Upsilon\left(\varkappa+\left\langle Q-c_{0}, h_{1}\right\rangle\right)^{N_{f}}|\Lambda / b|^{\langle Q, Q\rangle-2 \Delta\left(c_{0}\right)}$.

Let us compute the two-point function of the irregular puncture (A.113) with a generic vertex operator $\widehat{V}_{\alpha_{0}}$. Throughout the calculation, $\varkappa=\mathrm{i} \Lambda / b+O(1)$. Scale covariance and the explicit form (A.13) of the three-point function give

$$
\begin{gathered}
\frac{\Upsilon(\varkappa}{|\Lambda / b|^{2 \Delta\left(c_{0}\right)-\langle Q, Q\rangle} \mid}\left|\frac{x}{\Lambda}\right|^{2\left\langle\varkappa h_{1}, c_{0}-\varkappa h_{1}\right\rangle}\left\langle\widehat{V}_{\alpha_{0}}(\infty) \widehat{V}_{\varkappa h_{1}}\left(\frac{x}{-\mathrm{i} \Lambda}, \frac{\bar{x}}{\mathrm{i} \Lambda}\right) \widehat{V}_{c_{0}-\varkappa h_{1}}(0)\right\rangle \\
=\frac{|x / \Lambda|^{2\left\langle\varkappa h_{1}, c_{0}-\varkappa h_{1}\right\rangle-2 \Delta\left(\varkappa h_{1}\right)-2 \Delta\left(c_{0}-\varkappa h_{1}\right)+2 \Delta\left(\alpha_{0}\right)} \Upsilon\left(\varkappa+\left\langle Q-c_{0}, h_{1}\right\rangle\right)^{N_{f}}}{|\Lambda / b|^{2 \Delta\left(c_{0}\right)-\langle Q, Q\rangle} \prod_{s, t=1}^{N_{f}} \Upsilon\left(\frac{\varkappa}{N_{f}}+\left\langle Q-c_{0}+\varkappa h_{1}, h_{s}\right\rangle+\left\langle Q-\alpha_{0}, h_{t}\right\rangle\right)} \\
\sim \frac{|x / \Lambda|^{2 \Delta\left(\alpha_{0}\right)-2 \Delta\left(c_{0}\right)}|\Lambda / b|^{2 \Delta\left(\alpha_{0}\right)-\langle Q, Q\rangle}}{|\Lambda / b|^{2 \Delta\left(c_{0}\right)-\langle Q, Q\rangle} \prod_{s=2}^{N_{f}} \prod_{t=1}^{N_{f}} \Upsilon\left(\left\langle Q-c_{0}, h_{s}\right\rangle+\left\langle Q-\alpha_{0}, h_{t}\right\rangle\right)} .
\end{gathered}
$$

All powers of $\Lambda$ cancel, and we deduce that

$$
\left\langle\widehat{V}_{\alpha_{0}}(\infty) \widehat{\mathbb{V}}_{c_{0} ;-(x / b) h_{1},(\bar{x} / b) h_{1}}(0)\right\rangle=\frac{|x / b|^{2 \Delta\left(\alpha_{0}\right)-2 \Delta\left(c_{0}\right)}}{\prod_{s=2}^{N_{f}} \prod_{t=1}^{N_{f}} \Upsilon\left(\left\langle Q-c_{0}, h_{s}\right\rangle+\left\langle Q-\alpha_{0}, h_{t}\right\rangle\right)} .
$$

Note that the dependence on $|x / b|$ is as expected from the transformation (A.98) of rank 1 irregular punctures under a scaling. Both the OPE with $W_{N_{f}}$ currents, and the two-point function we have just computed, are finite, and independent of details such as the precise value of $\varkappa$ in the limit (A.113). This gives credence to our claim that collision limits $\mathbb{V}_{c_{0} ; \cdots ; c_{K}, \bar{c}_{K}}$ are finite and only depend on the $c_{j}$ and $\bar{c}_{j}$.

A similar calculation (not used in the main text) is the two-point function of a rank 1 irregular puncture (with $c_{1}$ and $\bar{c}_{1}$ collinear) and a semidegenerate operator:

$$
\begin{array}{r}
\left\langle\widehat{V}_{\varkappa h_{1}}(x, \bar{x}) \mathbb{V}_{c_{0} ; c_{1}, \bar{c}_{1}}(y, \bar{y})\right\rangle=\frac{\exp \left(\frac{\left\langle c_{0}-2 Q, c_{1}\right\rangle}{y-x}+\frac{\left\langle c_{0}-2 Q, \bar{c}_{1}\right\rangle}{\bar{y}-\bar{x}}\right)}{|x-y|^{4 \Delta\left(\varkappa h_{1}\right)} \hat{\mu}^{\left\langle c_{0}-2 Q, \rho\right\rangle} \prod_{s=1}^{N_{f}} \Upsilon\left(\frac{\varkappa}{N_{f}}+\left\langle 2 Q-c_{0}, h_{s}\right\rangle\right)} \\
\cdot \prod_{s<t}\left(-\left\langle c_{1}, h_{s}-h_{t}\right\rangle\left\langle\bar{c}_{1}, h_{s}-h_{t}\right\rangle\right)^{\left(\varkappa / N_{f}-\left\langle c_{0}-2 Q, h_{t}\right\rangle\right)\left(b+1 / b-\varkappa / N_{f}+\left\langle c_{0}-2 Q, h_{s}\right\rangle\right)}
\end{array}
$$

It is instructive to note how this expression is consistent with transformation properties (A.98) of rank 1 irregular punctures. Under a special conformal transformation $z \rightarrow w(z)$ which keeps $x$ and $y$ invariant, $c_{1}$ is scaled by $\partial_{z} w$ at $y$. Since at the fixed point $y$ one has $\frac{\partial_{z} w-1}{y-x}=\frac{\partial_{z}^{2} w}{2 \partial_{z} w}$, the exponential in (A.117) is shifted by $\frac{1}{2}\left\langle 2 Q-c_{0}, c_{1}\right\rangle \partial_{z}^{2} w / \partial_{z} w$, as required by (A.98). 


\section{B Vortex partition function dualities}

We prove here that the vortex partition functions of some dual theories are equal up to simple factors. The equalities are most easily seen through the matching with Toda CFT correlators, as done in the main text. However, the matching is not proven in all cases, so we proceed to establish the equalities directly using integral representations of the vortex partition functions. We cover the case of Seiberg duality for $\mathcal{N}=(2,2)$ SQCD in appendix B.1. We then add adjoint matter and a superpotential in appendix B.2: this includes as special cases the Seiberg duality for $\mathcal{N}=(2,2)^{*}$ SQCD, and the KutasovSchwimmer duality. The two appendices use similar ideas but are independent.

\section{B.1 SQCD vortex partition functions}

We focus first on the $S^{2}$ partition function of an $\mathcal{N}=(2,2)$ theory of a $\mathrm{U}(N)$ vector multiplet coupled to $N_{f}$ fundamental and $\widetilde{N_{f}}$ antifundamental chiral multiplets. Its expression can be decomposed as (2.58) into vortex partition functions [17, 18]. By symmetry we can assume that $\widetilde{N_{f}}<N_{f}$, or that $\widetilde{N_{f}}=N_{f}$ and $|z|<1$. The relevant vortex partition functions are then labeled by $N$-element subsets of $\llbracket 1, N_{f} \rrbracket$ and take the form

$$
Z_{\mathrm{v},\{p\}}(m, \widetilde{m}, x)=\sum_{k=0}^{\infty} x^{k} Z_{k,\{p\}}(m, \widetilde{m}),
$$

where $x=(-1)^{N_{f}+N-1} z$ and the $k$-vortex partition function is

$$
Z_{k,\{p\}}(m, \widetilde{m})=\sum_{k_{1}+\cdots+k_{N}=k} \prod_{j=1}^{N}\left[\frac{1}{k_{j} !} \frac{\prod_{s=1}^{\widetilde{N_{f}}}\left(-\mathrm{i} \widetilde{m}_{s}-\mathrm{i} m_{p_{j}}\right)_{k_{j}}}{\prod_{i \neq j}^{N}\left(\mathrm{i} m_{p_{i}}-\mathrm{i} m_{p_{j}}-k_{i}\right)_{k_{j}} \prod_{s \notin\{p\}}^{N_{f}}\left(1+\mathrm{i} m_{s}-\mathrm{i} m_{p_{j}}\right)_{k_{j}}}\right] .
$$

We prove that the vortex partition function is invariant under the Seiberg duality map $N^{D}=N_{f}-N,\{p\}^{D} \rightarrow\{p\}^{\complement}$ (the set complement), $m_{s}^{D}=\frac{\mathrm{i}}{2}-m_{s}, \widetilde{m}_{s}^{D}=\frac{\mathrm{i}}{2}-\widetilde{m}_{s}$, $z^{D}=(-1)^{\widetilde{N_{f}}} z$, up to a simple overall factor. This is based on the proof [17] that for $\widetilde{N_{f}} \leq N_{f}-2$ the $k$-vortex partition function is invariant. Since $Z_{k,\{p\}}$ depends analytically on the $m_{s}$ and $\widetilde{m}_{s}$, we only need to prove the equality when $R$-charges $\operatorname{Re}\left(-2 \mathrm{i} m_{s}\right)$ and $\operatorname{Re}\left(-2 \mathrm{i} \widetilde{m}_{s}\right)$ are between 0 and 1 ; the same is then true of the $R$-charges in the dual theory.

Consider a closed contour $C_{k}^{+}$which lies in the half-plane $\operatorname{Re}(\varphi)>-\frac{1}{2}$ and surrounds with a positive orientation all points $-\mathrm{i} m_{s}+\nu$ and $\frac{1}{2}+\mathrm{i} m_{s}+\nu$ for $1 \leq s \leq N_{f}$ and integer $0 \leq \nu<\kappa$. This set of points, which all have positive real part, is invariant under the duality map $-\mathrm{i} m_{s}^{D}=\frac{1}{2}+\mathrm{i} m_{s}$. The contour $C_{k}^{-}=-\frac{1}{2}-C_{k}^{+}$lies in the half-plane $\operatorname{Re}(\varphi)<0$ and surrounds with a positive orientation all points $-1-\mathrm{i} m_{s}-\nu$ and $-\frac{1}{2}+\mathrm{i} m_{s}-\nu$ for $1 \leq s \leq N_{f}$ and integer $0 \leq \nu<\kappa$. Define the contour integrals

$$
I_{k,\{p\}}^{ \pm}(m, \widetilde{m})=\frac{1}{k !} \int_{\left(C_{k}^{ \pm}\right)^{k}} \frac{\mathrm{d}^{k} \varphi}{(2 \pi \mathrm{i})^{k}} \prod_{\kappa \neq \lambda}^{k} \frac{\varphi_{\kappa}-\varphi_{\lambda}}{\varphi_{\kappa}-\varphi_{\lambda}-1} \prod_{\kappa=1}^{k} \frac{\prod_{s=1}^{\widetilde{N}_{f}}\left(\varphi_{\kappa}-\mathrm{i} \widetilde{m}_{s}\right)}{\prod_{s=1}^{N_{f}}\left(\varphi_{\kappa}+\mathrm{i} m_{s}+\delta_{s \notin\{p\}}\right)} .
$$

As we will see shortly, $I^{ \pm}$are essentially $k$-vortex partition functions of Seiberg dual theories (B.7). Given our choice of contours, the change of variables $\varphi \rightarrow \varphi^{D}=-\frac{1}{2}-\varphi$ maps 
$C_{k}^{ \pm} \rightarrow C_{k}^{\mp}$, and we find

$$
I_{k,\{p\}^{\mathrm{C}}}^{ \pm}\left(m^{D}, \widetilde{m}^{D}\right)=(-1)^{\left(1+\widetilde{N_{f}}+N_{f}\right) k} I_{k,\{p\}}^{\mp}(m, \widetilde{m}),
$$

where the sign comes from $\mathrm{d} \varphi^{D}=-\mathrm{d} \varphi, \varphi^{D}-\mathrm{i} \widetilde{m}^{D}=-(\varphi-\mathrm{i} \widetilde{m})$, and $\varphi^{D}+\mathrm{i} m_{s}^{D}+\delta_{s \in\{p\}}=$ $-\left(\varphi+\mathrm{i} m_{s}+\delta_{s \notin\{p\}}\right)$.

Poles of the integrand for which all $\operatorname{Re}\left(\varphi_{\kappa}\right)>-\frac{1}{2}$ are labeled by choices of $N$ integers $k_{s} \geq 0$ with $\sum_{s \in\{p\}} k_{s}=k$, such that the $\varphi_{\kappa}$ are given in some order by

$$
\left\{\varphi_{\kappa}\right\}=\left\{-\mathrm{i} m_{s}+\nu \mid s \in\{p\}, 0 \leq \nu<k_{s}\right\}
$$

hence $\left(C_{k}^{+}\right)^{k}$ surrounds precisely those poles. Similarly, poles with $\operatorname{Re}\left(\varphi_{\kappa}\right)<0$ are

$$
\left\{\varphi_{\kappa}\right\}=\left\{-1-\mathrm{i} m_{s}-\nu \mid s \notin\{p\}, 0 \leq \nu<k_{s}\right\},
$$

labeled by $N_{f}-N$ integers $k_{s} \geq 0$ for $s \notin\{p\}$, summing to $k$, and $\left(C_{k}^{-}\right)^{k}$ surrounds precisely those poles. For a given choice of $k_{1}+\cdots+k_{N}=k$, the residue at each of the $k$ ! points $\left\{\varphi_{\kappa}\right\}=\left\{-\mathrm{i} m_{p_{j}}+\nu \mid 1 \leq j \leq N, 0 \leq \nu<k_{j}\right\}$ reproduces the corresponding term in the $k$-vortex partition function (the factor $1 / k$ ! cancels the choice of ordering of $\varphi_{\kappa}$ ), hence the $k$-vortex partition functions are

$$
\begin{aligned}
Z_{k,\{p\}}(m, \widetilde{m}) & =I_{k,\{p\}}^{+}(m, \widetilde{m}) \\
Z_{k,\{p\}^{\mathrm{C}}}\left(m^{D}, \widetilde{m}^{D}\right) & =(-1)^{\left(1+\widetilde{N}_{f}+N_{f}\right) k} I_{k,\{p\}}^{-}(m, \widetilde{m}),
\end{aligned}
$$

where the dual relation derives from (B.4) or from summing residues at poles surrounded by $\left(C_{k}^{-}\right)^{k}$.

\section{B.1.1 SQCD with $\widetilde{N_{f}}<N_{f}$}

As long as $\widetilde{N_{f}} \leq N_{f}-2$, the integrand in (B.3) is regular at infinity, hence we can choose $C^{+}$along $-\frac{1}{4}+\mathrm{i} \mathbb{R}$, from i $\infty$ to $-\mathrm{i} \infty$ : then $C^{-}=-\frac{1}{2}-C^{+}$has the opposite orientation, and $I_{k,\{p\}}^{-}(m, \widetilde{m})=(-1)^{k} I_{k,\{p\}}^{+}(m, \widetilde{m})$. Therefore

$$
Z_{k,\{p\}^{\complement}}\left(m^{D}, \widetilde{m}^{D}\right)=(-1)^{\left(\widetilde{N_{f}}+N_{f}\right) k} Z_{k,\{p\}}(m, \widetilde{m}),
$$

hence vortex partition functions are equal:

$$
Z_{\mathrm{v},\{p\}^{\mathrm{C}}}\left(m^{D}, \widetilde{m}^{D}, x^{D}\right)=Z_{\mathrm{v},\{p\}}(m, \widetilde{m}, x),
$$

where $x^{D}=(-1)^{N_{f}+\widetilde{N_{f}}} x$ hence $z^{D}=(-1)^{\widetilde{N_{f}}} z$. This result strongly relies on our ability to reverse contours, that is, on the absence of poles at infinity for $\widetilde{N_{f}} \leq N_{f}-2$. For $\widetilde{N_{f}}=N_{f}-1$ or $\widetilde{N_{f}}=N_{f}$, we must take into account the contribution from infinity.

Consider first the case $\widetilde{N_{f}}=N_{f}-1$. We shift the pole at infinity to a finite position through the regulating factor $\mathrm{i} M /\left(\varphi_{\kappa}+\mathrm{i} M\right)$. This is equivalent to adding a fundamental chiral multiplet with twisted mass $M$ in the strip $0<\operatorname{Re}(-2 \mathrm{i} M)<1$. In the limit 
$|M| \rightarrow \infty$, the contours $\left(C_{k}^{ \pm}\right)^{k}$ only surround poles of the original integral, which are independent of $M$, and the regulator does not affect residues. Therefore,

$$
\begin{aligned}
I_{k,\{p\}}^{ \pm}(m, \widetilde{m})=\lim _{|M| \rightarrow \infty} \frac{1}{k !} \int_{\left(C_{k}^{ \pm}\right)^{k}} \frac{\mathrm{d}^{k} \varphi}{(2 \pi \mathrm{i})^{k}} & \left\{\prod_{\kappa \neq \lambda}^{k} \frac{\varphi_{\kappa}-\varphi_{\lambda}}{\varphi_{\kappa}-\varphi_{\lambda}-1}\right. \\
& \left.\cdot \prod_{\kappa=1}^{k}\left[\frac{\prod_{s=1}^{N_{f}-1}\left(\varphi_{\kappa}-\mathrm{i} \widetilde{m}_{s}\right)}{\prod_{s=1}^{N_{f}}\left(\varphi_{\kappa}+\mathrm{i} m_{s}+\delta_{s \notin\{p\}}\right)} \frac{\mathrm{i} M}{\varphi_{\kappa}+\mathrm{i} M}\right]\right\} .
\end{aligned}
$$

Poles of the integrand above with all $\operatorname{Re}\left(\varphi_{\kappa}\right)<0$ are identical to those of the non-regulated integral, hence integrating along the contour $-\frac{1}{4}+\mathrm{i} \mathbb{R}$ yields $I_{k,\{p\}}^{-}(m, \widetilde{m})$ by closing the contour towards $-\infty$. Closing the contour instead towards $+\infty$ surrounds poles at

$$
\left\{\varphi_{\kappa}\right\}=\left\{-\mathrm{i} m_{s}+\nu \mid s \in\{p\}, 0 \leq \nu<k_{s}\right\} \cup\{-\mathrm{i} M+\nu \mid 0 \leq \nu<l\},
$$

for each choice of non-negative integers $k_{s}$ for $s \in\{p\}$, and $l$, such that $l+\sum_{s \in\{p\}} k_{s}=k$. The residue at such a point is (factors of i $M$ cancel out)

$$
\frac{(-1)^{l}}{l !} \operatorname{res}_{\left\{\varphi_{\kappa} \mid 1 \leq \kappa \leq k-l\right\}}\left[\prod_{\kappa \neq \lambda}^{k-l} \frac{\varphi_{\kappa}-\varphi_{\lambda}}{\varphi_{\kappa}-\varphi_{\lambda}-1} \prod_{\kappa=1}^{k-l} \frac{\prod_{s=1}^{N_{f}-1}\left(\varphi_{\kappa}-\mathrm{i} \widetilde{m}_{s}\right)}{\prod_{s=1}^{N_{f}}\left(\varphi_{\kappa}+\mathrm{i} m_{s}+\delta_{s \notin\{p\}}\right)}\right],
$$

where the residue is precisely one of the contributions to $I_{k-l,\{p\}}^{+}(m, \widetilde{m})$. The contributions for a fixed $l$ combine into the full $(k-l)$-vortex partition function. All in all, using (B.7) $I_{k,\{p\}}^{-}(m, \widetilde{m})=Z_{k,\{p\}^{\mathrm{C}}}\left(m^{D}, \widetilde{m}^{D}\right)$ and $I_{k,\{p\}}^{+}=Z_{k,\{p\}}$ when $\widetilde{N}_{f}=N_{f}-1$,

$$
\begin{aligned}
Z_{k,\{p\}^{\mathrm{C}}}\left(m^{D}, \widetilde{m}^{D}\right) & =(-1)^{k} \sum_{l=0}^{k} \frac{(-1)^{l}}{l !} Z_{k-l,\{p\}}(m, \widetilde{m}), \\
Z_{\{p\}^{\mathrm{C}}}\left(m^{D}, \widetilde{m}^{D}, x^{D}\right) & =e^{-x} Z_{\{p\}}(m, \widetilde{m}, x) .
\end{aligned}
$$

Alternatively, the factor $e^{x}$ can be obtained from the case $N_{f}=\widetilde{N}_{f}+2$ (where there is no factor) by decoupling one of the fundamental chiral multiplets through the limit $\left|m_{N_{f}}\right| \rightarrow \infty$. For an arbitrary $N_{f}>\widetilde{N_{f}}$,

$$
Z_{k,\{p\}}^{N_{f}, \widetilde{N_{f}}} \sim \begin{cases}\left(\mathrm{i} m_{N_{f}}\right)^{-k} \sum_{l=0}^{k}(-1)^{l}\left(\begin{array}{c}
k \\
l
\end{array}\right)\left(-\mathrm{i} m_{N_{f}}\right)^{l\left(\widetilde{N_{f}}+2-N_{f}\right)} Z_{k-l,\{p\}}^{N_{f}-1, \widetilde{N_{f}}} & \text { if } N_{f} \in\{p\}, \\
\left(\mathrm{i} m_{N_{f}}\right)^{-k} Z_{k,\{p\}}^{N_{f}-1, \widetilde{N_{f}}} & \text { if } N_{f} \notin\{p\} .\end{cases}
$$

If $N_{f} \geq \widetilde{N_{f}}+3$, terms other than $l=0$ in the sum are of a lower order, thus $Z_{k,\{p\}}^{N_{f}, \widetilde{N}_{f}} \sim\left(\mathrm{i} m_{N_{f}}\right)^{-k} Z_{k,\{p\}}^{N_{f}-1, \widetilde{N_{f}}}$, consistent with the equality (B.8) of Seiberg-dual vortex partition functions in those cases. If $N_{f}=\widetilde{N}_{f}+2$, we find

$$
Z_{\{p\}}^{\widetilde{N_{f}}+2, \widetilde{N_{f}}}\left(\mathrm{i} m_{N_{f}} x\right) \sim e^{-x \delta_{N_{f}} \in\{p\}} Z_{\{p\}}^{\widetilde{N_{f}}+1, \widetilde{N_{f}}}(x) .
$$


Exactly one of two Seiberg-dual vortex partition functions exhibits this exponential factor, and with opposite signs since $\mathrm{i} m_{N_{f}}^{D} \sim-\mathrm{i} m_{N_{f}}$. Starting from the Seiberg duality relation (B.9) for $N_{f} \geq \widetilde{N}_{f}+2$, we thus obtain the exponential factor in (B.14) for $N_{f}=\widetilde{N}_{f}+1$. Unfortunately, the same technique fails to reach the case $N_{f}=\widetilde{N}_{f}$, because terms beyond (B.15) contribute to the limit $\left|m_{N_{f}}\right| \rightarrow \infty$ (with $x / m_{N_{f}}$ kept constant). We avoid this issue in the contour integral approach by introducing different parameters for each occurrence of $m_{N_{f}}$, as we now see.

\section{B.1.2 SQCD with $\widetilde{N_{f}}=N_{f}$}

When $\widetilde{N_{f}}=N_{f}$, we regulate using $\prod_{\kappa=1}^{k}\left[-\left(\mathrm{i} M_{\kappa}\right)^{2} /\left(\varphi_{\kappa}^{2}-\left(\mathrm{i} M_{\kappa}\right)^{2}\right)\right]$ with $M_{\kappa}$ real for simplicity. This factor is similar to the contribution from two fundamental chiral multiplets with opposite twisted masses, but importantly we let the parameter $M_{\kappa}$ depend on $\kappa$. In fact, we will consider the limit where masses have different scales, $1 \ll\left|M_{1}\right| \ll \cdots \ll\left|M_{k}\right|$, as this simplifies the expansion of residues. For large enough $\left|M_{\kappa}\right|$, the additional poles lie outside the contours $\left(C_{k}^{ \pm}\right)^{k}$, and the regulating factor tends to 1 when evaluated on the contour (or at poles it encloses), thus

$$
\begin{aligned}
I_{k,\{p\}}^{ \pm}(m, \tilde{m})=\lim _{\left|M_{\kappa}\right| \rightarrow \infty} \frac{1}{k !} \int_{\left(C_{k}^{ \pm}\right)^{k}} \frac{\mathrm{d}^{k} \varphi}{(2 \pi \mathrm{i})^{k}}\left\{\prod_{\kappa=1}^{k}\left[\frac{-\left(\mathrm{i} M_{\kappa}\right)^{2}}{\varphi_{\kappa}^{2}-\left(\mathrm{i} M_{\kappa}\right)^{2}}\right]\right. \\
\left.\cdot \prod_{\kappa \neq \lambda}^{k}\left[\frac{\varphi_{\kappa}-\varphi_{\lambda}}{\varphi_{\kappa}-\varphi_{\lambda}-1}\right] \prod_{\kappa=1}^{k} \prod_{s=1}^{N_{f}}\left[\frac{\varphi_{\kappa}-\mathrm{i} \widetilde{m}_{s}}{\varphi_{\kappa}+\mathrm{i} m_{s}+\delta_{s \notin\{p\}}}\right]\right\}
\end{aligned}
$$

Poles of the integrand above with all $\operatorname{Re}\left(\varphi_{\kappa}\right) \leq-\frac{1}{4}$ are identical to those of the nonregulated integral, hence integrating along the contour $-\frac{1}{4}+\mathrm{i} \mathbb{R}$ yields $Z_{k,\{p\}}^{-}(m, \widetilde{m})$ by closing the contour towards $-\infty$.

Closing the contour instead towards $+\infty$ surrounds numerous poles:

$$
\left\{\varphi_{\kappa}\right\}=\left\{-\mathrm{i} m_{s}+\mu \mid s \in\{p\}, 0 \leq \mu<k_{s}\right\} \cup\left\{\epsilon_{\kappa} \mathrm{i} M_{\kappa}+\nu \mid \kappa \in K, 0 \leq \nu<l_{\kappa}\right\}
$$

where $K$ is the set of $1 \leq \kappa \leq k$ such that $\varphi_{\kappa}=\epsilon_{\kappa} \mathrm{i} M_{\kappa}$ for some sign $\epsilon_{\kappa}= \pm 1$, and where the integers $k_{s} \geq 0$ for $s \in\{p\}$ and $l_{\kappa}>0$ for $\kappa \in K$ sum to $k$. To specify a pole completely, one needs to know $\left\{K, \epsilon_{\kappa}, l_{\kappa}, k_{s}\right\}$, but also which component of $\varphi$ is equal to each $-\mathrm{i} m_{s}+\mu$ and each $\epsilon_{\kappa} \mathrm{i} M_{\kappa}+\nu$. This is encoded in maps $\sigma$ and $\tau$ such that

$$
\varphi_{\sigma(s, \mu)}=-\mathrm{i} m_{s}+\mu \quad \text { and } \quad \varphi_{\tau(\kappa, \nu)}=\epsilon_{\kappa} \mathrm{i} M_{\kappa}+\nu
$$

Note that $\tau(\kappa, \nu)=\kappa$ if and only if $\nu=0$.

We expand the residue at the pole defined by $\left\{K, \epsilon_{\kappa}, l_{\kappa}, k_{s}, \sigma, \tau\right\}$ in the limit $1 \ll$ 


$$
\begin{aligned}
&\left|M_{1}\right| \ll \cdots \ll\left|M_{k}\right|: \\
& \frac{1}{k !} \prod_{\kappa \in K}\left[\frac{-\epsilon_{\kappa} \mathrm{i} M_{\kappa}}{2} \prod_{\nu=1}^{l_{\kappa}-1}\left[\frac{-\left(\mathrm{i} M_{\tau(\kappa, \nu)}\right)^{2}}{\left(\epsilon_{\kappa} \mathrm{i} M_{\kappa}+\nu\right)^{2}-\left(\mathrm{i} M_{\tau(\kappa, \nu)}\right)^{2}}\right]\right] \prod_{s \in\{p\}} \prod_{\mu=0}^{k_{s}-1}\left[1+O\left(\frac{1}{M_{\sigma(s, \mu)}^{2}}\right)\right] \\
& \quad \prod_{\kappa \in K}\left[1-\frac{l_{\kappa} \Sigma}{\epsilon_{\kappa} \mathrm{i} M_{\kappa}}+O\left(\frac{1}{M_{\kappa}^{2}}\right)\right] \prod_{\kappa \in K}\left[1+O\left(\frac{1}{M_{\kappa}^{2}}\right)\right] \prod_{\kappa \in K}\left[\frac{1}{l_{\kappa}}\right] \\
& \quad \prod_{\varphi_{\sigma(s, \mu)}=-\mathrm{i} m_{s}+\mu}\left\{\prod_{\kappa \neq \lambda \in\{\sigma(s, \mu)\}}\left[\frac{\varphi_{\kappa}-\varphi_{\lambda}}{\varphi_{\kappa}-\varphi_{\lambda}-1}\right] \prod_{\kappa \in\{\sigma(s, \mu)\}} \prod_{t=1}^{N_{f}}\left[\frac{\varphi_{\kappa}-\mathrm{i} \widetilde{m}_{t}}{\varphi_{\kappa}+\mathrm{i} m_{t}+\delta_{t \notin\{p\}}}\right]\right\} .
\end{aligned}
$$

The first line consists of all factors coming from the regulator; the next factor comes from $\left(\varphi_{\tau(\ldots)}-\mathrm{i} \widetilde{m}_{s}\right) /\left(\varphi_{\tau(\ldots)}+\mathrm{i} m_{s}+\delta_{s \notin\{p\}}\right)$ and involves

$$
\Sigma=\sum_{s=1}^{N_{f}}\left(\mathrm{i} \widetilde{m}_{s}+\mathrm{i} m_{s}+\delta_{s \notin\{p\}}\right) ;
$$

the following two factors come from the ratio $(\varphi-\varphi) /(\varphi-\varphi-1)$ where either one or both components of $\varphi$ take the form $\varphi_{\tau(\kappa, \mu)}$; the last line consists of all finite factors, independent of the $M_{\kappa}$, which organize themselves into a residue along the components $\varphi_{\sigma(s, \mu)}$. A useful simplification is

$$
\frac{-\left(\mathrm{i} M_{\tau(\kappa, \nu)}\right)^{2}}{\left(\epsilon_{\kappa} \mathrm{i} M_{\kappa}+\nu\right)^{2}-\left(\mathrm{i} M_{\tau(\kappa, \nu)}\right)^{2}} \sim \begin{cases}-M_{\tau(\kappa, \nu)}^{2} M_{\kappa}^{-2} & \text { if } \tau(\kappa, \nu)<\kappa \\ 1 & \text { if } \tau(\kappa, \nu)>\kappa\end{cases}
$$

On its own, the residue (B.20) grows like $\prod_{\kappa}\left(-\epsilon_{\kappa} \mathrm{i} M_{\kappa}\right)$, but we will see that the sum over all possible choices of the signs $\epsilon_{\kappa}$ (keeping $\left\{K, l_{\kappa}, k_{s}, \sigma, \tau\right\}$ fixed) has a finite limit. More precisely, starting from $\lambda=k$, and all the way down to $\lambda=1$, we sum over $\epsilon_{\lambda}= \pm 1$ (if $\lambda \in K)$ and take the limit $\left|M_{\lambda}\right| \rightarrow \infty$. At each step there are three cases. If $\lambda=\sigma(s, \mu)$, the twisted mass appears only in a factor $1+O\left(1 / M_{\lambda}^{2}\right)$, which thus drops out. If $\lambda=\tau(\kappa, \nu)>\kappa$, then the factor (B.22) containing $M_{\lambda}$ drops out. The case $\lambda=\tau(\kappa, \nu)<\kappa$ does not appear, as we see shortly. Finally, if $\lambda \in K$, several factors contain $M_{\lambda}$ :

$$
\frac{-\epsilon_{\lambda} \mathrm{i} M_{\lambda}}{2} \prod_{\substack{1 \leq \nu<l_{\lambda} \\ \tau(\lambda, \nu)<\lambda}}\left[\frac{-\left(\mathrm{i} M_{\tau(\lambda, \nu)}\right)^{2}}{\left(\epsilon_{\lambda} \mathrm{i} M_{\lambda}+\nu\right)^{2}-\left(\mathrm{i} M_{\tau(\lambda, \nu)}\right)^{2}}\right]\left[1-\frac{l_{\lambda} \Sigma}{\epsilon_{\lambda} \mathrm{i} M_{\lambda}}+O\left(\frac{1}{M_{\lambda}^{2}}\right)\right]\left[1+O\left(\frac{1}{M_{\lambda}^{2}}\right)\right] .
$$

This expression vanishes in the limit $\left|M_{\lambda}\right| \rightarrow \infty$ if any $\tau(\lambda, \nu)<\lambda$, thus only poles for which all $\tau(\lambda, \nu) \geq \lambda$ contribute in the limit we consider. Otherwise, the expression above is $\frac{1}{2}\left(-\epsilon_{\lambda} \mathrm{i} M_{\lambda}+l_{\lambda} \Sigma+O\left(1 / M_{\lambda}\right)\right)$, whose sum over $\epsilon_{\lambda}= \pm 1$ is the finite result $l_{\lambda} \Sigma$. All in all, the sum over all choices of signs $\epsilon$ of the residue at the pole defined by $\left\{K, \epsilon_{\kappa}, l_{\kappa}, k_{s}, \sigma, \tau\right\}$ has a finite limit

$$
\frac{1}{k !} \Sigma^{\# K} \operatorname{res}_{\varphi_{\sigma(s, \mu)}=-\mathrm{i} m_{s}+\mu}\left\{\prod_{\kappa \neq \lambda \in\{\sigma(s, \mu)\}}\left[\frac{\varphi_{\kappa}-\varphi_{\lambda}}{\varphi_{\kappa}-\varphi_{\lambda}-1}\right] \prod_{\kappa \in\{\sigma(s, \mu)\}} \prod_{t=1}^{N_{f}}\left[\frac{\varphi_{\kappa}-\mathrm{i} \widetilde{m}_{t}}{\varphi_{\kappa}+\mathrm{i} m_{t}+\delta_{t \notin\{p\}}}\right]\right\},
$$

which turns out to only depends on the number $\# K$ of elements in $K$ and on the $k_{s}$. 
We must now sum this expression over all choices of sets $K$, of integers $l_{\kappa}>0$ and $k_{s} \geq$ 0 , and of indices $\sigma(s, \mu)$ and $\tau(\kappa, \nu)>\kappa$. The choice of $\left\{K, l_{\kappa}, k_{s}, \sigma, \tau\right\}$ can be split into a choice of $\left\{K, l_{\kappa}, \tau\right\}$ followed by a choice of integers $k_{s} \geq 0$ summing to $k-l$, where $l=\sum_{\kappa \in K} l_{\kappa}$, and finally a choice of $\sigma$ labeling the complement of $T=\{\tau(\cdot, \cdot)\}$ by pairs $(s, \mu)$. This last choice does not affect the residue, hence contributes a factor of $(k-l)$ !. The sum over $\left\{k_{s}\right\}$ (summing to $\left.k-l\right)$ of the residue in (B.24) yields the $(k-l)$-vortex partition function. Thus,

$$
Z_{k,\{p\}}^{-}(m, \widetilde{m})=(-1)^{k} \sum_{l=0}^{\infty}\left[\frac{(k-l) !}{k !} \sum_{T \mid \# T=l} \sum_{K \subseteq T} \sum_{\left\{l_{k} \geq 1\right\}} \sum_{\tau}\left[\Sigma^{\# K}\right] Z_{k-l,\{p\}}^{+}(m, \widetilde{m})\right] .
$$

The number of choices of $\left\{K, l_{\kappa}, \tau\right\}$ with a given $\# K$ only depends on the size $l=\# T$, thus the choice of $T$ contributes a factor $k ! /[l !(k-l) !]$. At this point, we could conclude by noting that we expressed $Z_{k,\{p\}}^{-}(m, \widetilde{m})$ in terms of the $Z_{k-l,\{p\}}^{+}(m, \widetilde{m})$ with coefficients depending only on $l$ and the combination $\Sigma$ of twisted masses, and neither on $N_{f}$ nor on $N$. The coefficients can thus be obtained through the special case $\widetilde{N_{f}}=N_{f}=1, N=0$, for which computations are elementary, leading to a Seiberg duality relation valid for arbitrary $\widetilde{N_{f}}=N_{f}$ and $N$.

For completeness, we go through the combinatorical exercise. Since only $l=\# T$ affects the counting, we can fix $T=\llbracket 1, l \rrbracket$ to simplify the discussion. Define the map $v: T \rightarrow T$ such that for each $\kappa \in K, v(\kappa)=\kappa$ and $v(\tau(\kappa, \nu))=\max \{\tau(\kappa, \mu) \mid 0 \leq \mu<\nu, \tau(\kappa, \mu)<$ $\tau(\kappa, \nu)\}$ for $\nu>0$. The data of $K \subseteq T=\llbracket 1, l \rrbracket$ and $v: T \rightarrow T$ with $v(\kappa)=\kappa$ for $\kappa \in K$ and $v(\lambda)<\lambda$ for $\lambda \in T \backslash K$ is in fact equivalent to that of $\left\{K, l_{\kappa}, \tau\right\}$. There are $\prod_{\lambda \in T \backslash K}(\lambda-1)$ maps $v$, hence

$$
\begin{aligned}
Z_{k,\{p\}}^{-}(m, \widetilde{m}) & =(-1)^{k} \sum_{l=0}^{\infty}\left\{\frac{1}{l !}\left(\sum_{K \subseteq \llbracket 1, l \rrbracket} \Sigma^{\# K} \prod_{\lambda \in \llbracket 1, l \rrbracket \backslash K}(\lambda-1)\right) Z_{k-l,\{p\}}^{+}(m, \widetilde{m})\right\} \\
& =(-1)^{k} \sum_{l=0}^{\infty} \frac{(\Sigma)_{l}}{l !} Z_{k-l,\{p\}}^{+}(m, \widetilde{m}),
\end{aligned}
$$

where $(\Sigma)_{l}=\Sigma \cdots(\Sigma+l-1)$ is the Pochhammer symbol. From this, we can finally deduce the Seiberg duality relation

$$
Z_{\{p\}^{\mathrm{C}}}\left(m^{D}, \widetilde{m}^{D}, x^{D}\right)=(1-x)^{-\Sigma} Z_{\{p\}}(m, \widetilde{m}, x),
$$

with $x^{D}=x$ hence $z^{D}=(-1)^{\widetilde{N_{f}}} z$, and where we recall $\Sigma=\sum_{s=1}^{N_{f}}\left(\mathrm{i} \widetilde{m}_{s}+\mathrm{i} m_{s}\right)+N_{f}-N$. This relation precisely matches that obtained in the main text as Toda conjugation, in particular the exponent (3.4).

\section{B.2 SQCDA vortex partition functions}

We now adapt the proof to $\mathcal{N}=(2,2)$ SQCDA theories with a superpotential. The field content consists of a vector multiplet coupled to one adjoint chiral multiplet $X, N_{f}$ fundamental chiral multiplets $q_{s}$, and $N_{f}$ antifundamental chiral multiplets $\widetilde{q}_{s}$. As in sec- 
tion 2.3.3 we consider two cases: the superpotential $W=\sum_{t=1}^{N_{f}} \widetilde{q}_{t} X^{l_{t}} q_{t}$ and the superpotential $W=\operatorname{Tr} X^{l+1}$ for integers $l_{t}, l \geq 0$. Both choices exhibit common features, with $l_{t}$ replaced by $l$ for the second superpotential.

In sections 3.2.1 and 3.2.2, we find that pairs of such theories with gauge groups $\mathrm{U}(N)$ and $\mathrm{U}\left(N^{D}\right)$ are dual, using symmetries of Toda CFT correlators. Parameters are mapped as follows: $m_{X}^{D}=m_{X}, N^{D}=L-N$ with $L=\sum_{t=1}^{N_{f}} l_{t}$, and

$$
\begin{aligned}
& m_{t}^{D}=m_{t}, \quad \widetilde{m}_{t}^{D}=m_{t}^{D}, \quad z^{D}=z^{-1} \quad \text { for } W=\sum_{t=1}^{N_{f}} \widetilde{q}_{t} X^{l_{t}} q_{t} \\
& m_{t}^{D}=m_{X}-m_{t}, \quad \widetilde{m}_{t}^{D}=m_{X}-\widetilde{m}_{t}, \quad z^{D}=z \quad \text { for } W=\operatorname{Tr} X^{l+1} .
\end{aligned}
$$

Higgs branch vacua of the $\mathrm{U}(N)$ theory are labeled by integers $0 \leq n_{t} \leq l_{t}$ with sum $N$. Those are in a natural bijection $n_{t}^{D}=l_{t}-n_{t}$ to integers $0 \leq n_{t}^{D} \leq l_{t}$ with sum $L-N$, which label Higgs branch vacua of the dual theory. We compare classical and one-loop contributions in sections 3.2.1 and 3.2.2. We now prove the relations (B.57) and (B.58) between the vortex partition functions of the $\mathrm{U}(N)$ theory in the vacuum $\left\{n_{t}\right\}$ and of the $\mathrm{U}(L-N)$ theory in the vacuum $\left\{l_{t}-n_{t}\right\}$. As in the main text, $y=(-1)^{N_{f}} z$.

\section{B.2.1 Preliminary result for $N_{f}=1$}

Later on, we prove that dual vortex partition functions are equal up to some factor which only depends on very little data. To fix this factor, we will use the simple case of $N_{f}=1$ SQCDA with $1+\mathrm{i} m_{1}+\mathrm{i} \widetilde{m}_{1}+N \mathrm{i} m_{X}=0$, which we consider now. Its unique vacuum has $n_{1}=N$, and we prove that $Z_{\mathrm{v},\{N\}}(y)=(1-y)^{-N\left(1+\mathrm{i} m_{X}\right)}$. By analyticity, it is enough to show this when $\operatorname{Re}\left(\mathrm{i} \widetilde{m}_{1}\right)<0<\operatorname{Re}\left(-\mathrm{i} m_{1}\right)<\operatorname{Re}\left(-\mathrm{i} m_{X}\right)$.

The vortex partition function, given by the series (2.97), has a Mellin-Barnes integral representation

$$
\begin{aligned}
& Z_{\mathrm{v},\{N\}}(y)=\sum_{\left\{k_{\mu} \geq 0\right\}} y^{\sum k_{\mu}} \prod_{\mu, \nu=0}^{N-1} \frac{\left((\nu-\mu-1) \mathrm{i} m_{X}-k_{\nu}\right)_{k_{\mu}}}{\left((\nu-\mu) \mathrm{i} m_{X}-k_{\nu}\right)_{k_{\mu}}} \\
& =(-y)^{N \mathrm{i} m_{1}+\frac{1}{2}(N-1) N \mathrm{i} m_{X}} \prod_{\mu=1}^{N} \frac{\sin \pi\left(-\mu \mathrm{i} m_{X}\right)}{\pi} \\
& \cdot \frac{1}{N !} \int_{\mathbb{R}^{N}} \frac{\mathrm{d}^{N} \sigma}{(2 \pi)^{N}}(-y)^{\operatorname{Tr} \mathrm{i} \sigma} \prod_{j=1}^{N}\left[\Gamma\left(-\mathrm{i} m_{1}-\mathrm{i} \sigma_{j}\right) \Gamma\left(-\mathrm{i} \widetilde{m}_{1}+\mathrm{i} \sigma_{j}\right)\right] \frac{\prod_{i, j=1}^{N} \Gamma\left(\mathrm{i} \sigma_{i}-\mathrm{i} \sigma_{j}-\mathrm{i} m_{X}\right)}{\prod_{i \neq j}^{N} \Gamma\left(\mathrm{i} \sigma_{i}-\mathrm{i} \sigma_{j}\right)}
\end{aligned}
$$

which analytically continues $Z_{\mathrm{v},\{N\}}(y)$ from the unit disc to $y \notin \mathbb{R}_{\geq 0}$. Closing contours towards i $\infty$ yields a similar relation for $|y|>1$, with $m_{1} \leftrightarrow \widetilde{m}_{1}$ and $y \rightarrow y^{-1}$. Hence, the analytic continuations obey

$$
Z_{\mathrm{v},\{N\}}(y)=(-y)^{-N\left(1+\mathrm{i} m_{X}\right)} Z_{\mathrm{v},\{N\}}\left(y^{-1}\right) .
$$

The function $(1-y)^{N\left(1+\mathrm{i} m_{X}\right)} Z_{\mathrm{v},\{N\}}(y)$ is thus analytic on the Riemann sphere away from $y=1$. Furthermore, we can bound it by a power of $|1-y|$ in two pairs of angular sectors centered at $y=1$, whose union is a neighborhood of $y=1$. 
The first angular sector is defined by $|1-y|<M(1-|y|)$ for some $M>0$ and is contained in the open unit disc. The coefficients in the series (B.30) grow at most polynomially in the exponent $\sum_{\mu} k_{\mu}$ of $y$, and the number of terms contributing for a given power of $y$ also grows polynomially. Hence,

$$
\left|Z_{\mathrm{v},\{N\}}(y)\right| \leq \sum_{k \geq 0} C_{1}(k+1)_{C_{2}}|y|^{k}=C_{2} ! C_{1}(1-|y|)^{-1-C_{2}}
$$

for some $C_{1}, C_{2}>0$ which do not depend on $y$. Thus $|1-y|^{1+C_{2}} Z_{\mathrm{v},\{N\}}(y)$ is bounded in each sector $|1-y|<M(1-|y|)$. By the symmetry $y \rightarrow y^{-1}$, the function is also bounded in a similar sector $|y-1|<M(|y|-1)$. We have thus probed the function away from the unit circle.

The next pair of sectors is probed using the Mellin-Barnes representation (B.31), which converges away from the real axis. Set $y=r e^{\mathrm{\epsilon i} \theta}$ with $\frac{1}{2}<r<2$ (to avoid $\left.\{0, \infty\}\right), \epsilon= \pm 1$, and $0<\theta<\pi$ (that is, $y \notin \mathbb{R}$ ). Then

$$
\left|(-y)^{\mathrm{i} \sigma_{j}}\right|=e^{\epsilon(\pi-\theta) \sigma_{j}} \leq e^{(\pi-\theta)|\sigma|} .
$$

For some large enough $C_{1}, C_{2}>0$ which depend on the twisted masses, we have

$$
\left|\frac{\Gamma\left(-\mathrm{i} m_{1}-\mathrm{i} \sigma_{j}\right)}{\Gamma\left(1+\mathrm{i} \widetilde{m}_{1}-\mathrm{i} \sigma_{j}\right)}\right|<C_{1}(|\sigma|+1)^{N \operatorname{Re}\left(\mathrm{i} m_{X}\right)},\left|\frac{\Gamma\left(\mathrm{i} \sigma_{i}-\mathrm{i} \sigma_{j}-\mathrm{i} m_{X}\right)}{\Gamma\left(\mathrm{i} \sigma_{i}-\mathrm{i} \sigma_{j}\right)}\right|<C_{2}(|\sigma|+1)^{\operatorname{Re}\left(-\mathrm{i} m_{X}\right)}
$$

for all $\sigma$, where $|\sigma|=\left(\sum_{i=1}^{N}\left|\sigma_{i}\right|^{2}\right)^{1 / 2}$ is larger than all $\left|\sigma_{j}\right|$ and all $\left|\sigma_{i}-\sigma_{j}\right|$. The inequalities rely on the asymptotics $\Gamma(a+\mathrm{i} v) / \Gamma(b+\mathrm{i} v) \sim(\mathrm{i} v)^{a-b}$ as $v \rightarrow \pm \infty$, and the continuity of both ratios of Gamma functions. Since $0<\operatorname{Im}\left(\widetilde{m}_{1}\right)<1$, we also have

$$
\left|\Gamma\left(1+\mathrm{i} \widetilde{m}_{1}-\mathrm{i} \sigma_{j}\right) \Gamma\left(-\mathrm{i} \widetilde{m}_{1}+\mathrm{i} \sigma_{j}\right)\right| \leq \frac{2 \pi e^{-\pi\left|\sigma_{j}-\operatorname{Re}\left(\widetilde{m}_{1}\right)\right|}}{\left|\sin \left(\pi \operatorname{Im}\left(\widetilde{m}_{1}\right)\right)\right|}<C_{3} e^{-\pi|\sigma|}
$$

for some $\widetilde{m}_{1}$-dependent $C_{3}>0$. Combining the bounds into (B.31) yields

$$
\left|Z_{\mathrm{v},\{N\}}(y)\right| \leq C_{4} \int_{\mathbb{R}^{N}} \mathrm{~d}^{N} \sigma e^{-N \theta|\sigma|}(|\sigma|+1)^{N \operatorname{Re}\left(\mathrm{i} m_{X}\right)}
$$

for some $C_{4}>0$. Switching to polar coordinates, letting $\tau=\theta(|\sigma|+1)$, and bounding $(\tau-\theta)^{N-1}<\tau^{N-1}$ leads to

$$
\left|\theta^{N\left(1+\mathrm{i} m_{X}\right)} Z_{\mathrm{v},\{N\}}(y)\right| \leq C_{5} \int_{\theta}^{\infty} \mathrm{d} \tau e^{-N \tau} \tau^{N \operatorname{Re}\left(\mathrm{i} m_{X}\right)+N-1} \leq C_{6}
$$

for some $C_{5}, C_{6}>0$. In any angular sector centered at $y=1$ and away from the real axis, $|1-y|$ is bounded by some multiple of $\theta=\arg (y)$, hence $(1-y)^{N\left(1+\mathrm{i} m_{X}\right)} Z_{\mathrm{v},\{N\}}(y)$ is bounded both above and below the real axis.

We have bounded the function $(1-y)^{N\left(1+\mathrm{i} m_{X}\right)} Z_{\mathrm{v},\{N\}}(y)$ by a power of $|1-y|$ in a neighborhood of $y=1$. Since the function is analytic away from 1 , it takes the form $P(y) /(1-y)^{n}$, where $P(y)$ is a polynomial of degree at most $n \geq 0$. In the second pair of sectors, we found that the function is bounded as $y \rightarrow 1$, thus $n=0$ and the function is the constant $(1-y)^{N\left(1+\mathrm{i} m_{X}\right)} Z_{\mathrm{v},\{N\}}(y)=Z_{\mathrm{v},\{N\}}(0)=1$. 


\section{B.2.2 Proof for SQCDA}

Let us move on to the proof per se. We start with the vortex partition function (2.97) of the $\mathrm{U}(N)$ SQCDA theory in a given Higgs branch vacuum $\left\{n_{s}\right\}$. The terms of this series in $y=(-1)^{N_{f}} z$ are labeled by integer vorticities $k_{s \mu} \geq 0$ for $1 \leq s \leq N_{f}$ and $0 \leq \mu<n_{s}$ :

$$
Z_{\mathrm{v},\left\{n_{s}\right\}}(y)=\sum_{k \geq 0} y^{k} Z_{\mathrm{v},\left\{n_{s}\right\}, k}=\sum_{k \geq 0} y^{k} \sum_{\sum k_{s \mu}=k} V_{\left\{n_{s}\right\}}^{\left\{k_{s \mu}\right\}} .
$$

The contribution $V_{\left\{n_{s}\right\}}^{\left\{k_{s \mu}\right\}}$ for a given choice of vorticities is a ratio of Pochhammer symbols, which we massage using the identity $(1-x-k)_{k-j}=(-1)^{k-j}(x)_{k} /(x)_{j}$ into

$$
V_{\left\{n_{s}\right\}}^{\left\{k_{s \mu}\right\}}=\prod_{(s, \mu) \in I}\left[\prod_{t=1}^{N_{f}} \frac{\left(-\mathrm{i} \tilde{m}_{t}-\mathrm{i} m_{s \mu}\right)_{k_{s \mu}}}{\left(1+\mathrm{i} m_{t}+n_{t} \mathrm{i} m_{X}-\mathrm{i} m_{s \mu}\right)_{k_{s \mu}}} \prod_{(t, \nu) \in I} \frac{\left(\mathrm{i} m_{t \nu}-\mathrm{i} m_{s \mu}-\mathrm{i} m_{X}-k_{t \nu}\right)_{k_{s \mu}}}{\left(\mathrm{i} m_{t \nu}-\mathrm{i} m_{s \mu}-k_{t \nu}\right)_{k_{s \mu}}}\right] .
$$

Here, $m_{s}, \widetilde{m}_{s}$, and $m_{X}$ are complexified twisted masses of the chiral multiplets, we denote $m_{s \mu}=m_{s}+\mu m_{X}$, the products range over $I=\left\{(s, \mu) \mid 1 \leq s \leq N_{f}, 0 \leq \mu<n_{s}\right\}$, and we have swapped $(s, \mu) \leftrightarrow(t, \nu)$ compared to (2.97). Using that

$$
\prod_{(s, \mu) \in I} \prod_{(t, \nu) \in I} \frac{\left(\mathrm{i} m_{t \nu}-\mathrm{i} m_{s \mu}-A-k_{t \nu}\right)_{k_{s \mu}}}{\left(\mathrm{i} m_{t \nu}-\mathrm{i} m_{s \mu}-A\right)_{k_{s \mu}}}=\prod_{\substack{(s, \mu) \in I \\ 0 \leq i<k_{s \mu}}} \prod_{\substack{(t, \nu) \in I \\ 0 \leq j<k_{t \nu}}} \frac{\mathrm{i} m_{t \nu}-j-\mathrm{i} m_{s \mu}+i-A-1}{\mathrm{i} m_{t \nu}-j-\mathrm{i} m_{s \mu}+i-A}
$$

for a generic $A \in \mathbb{C}$, we can express $V_{\left\{n_{s}\right\}}^{\left\{k_{s \mu}\right\}}$ in terms of the combinations $-\mathrm{i} m_{s \mu}+i$ for $(s, \mu) \in I$ and $0 \leq i<k_{s \mu}$. We find that $(-1)^{k} V_{\left\{n_{s}\right\}}^{\left\{k_{s \mu}\right\}}$ is the residue at $\left\{\varphi_{\kappa}\right\}=\left\{-\mathrm{i} m_{s \mu}+i \mid\right.$ $\left.0 \leq i<k_{s \mu}\right\}$ of the integrand in (B.42) below, after $\left|M_{\kappa}\right| \rightarrow \infty$.

The discussion above leads us to the contour integral ( $I_{0}=1$ is an empty product)

$$
\begin{aligned}
& I_{k}=\lim _{\left|M_{1}\right| \rightarrow \infty} \cdots \lim _{\left|M_{k}\right| \rightarrow \infty} \frac{1}{k !} \prod_{\kappa=1}^{k}\left[\int_{-\mathrm{i} \infty}^{\mathrm{i} \infty} \frac{\mathrm{d} \varphi_{\kappa}}{2 \pi \mathrm{i}}\right]\left\{\prod_{\kappa=1}^{k} \frac{-\left(\mathrm{i} M_{\kappa}\right)^{2}}{\left(\varphi_{\kappa}-\frac{1}{2}\right)^{2}-\left(\mathrm{i} M_{\kappa}\right)^{2}} \prod_{\kappa \neq \lambda}^{k} \frac{\varphi_{\kappa}-\varphi_{\lambda}}{\varphi_{\kappa}-\varphi_{\lambda}-1}\right. \\
& \left.\cdot \prod_{\kappa, \lambda=1}^{k} \frac{\varphi_{\kappa}-\varphi_{\lambda}-1-\mathrm{i} m_{X}}{\varphi_{\kappa}-\varphi_{\lambda}-\mathrm{i} m_{X}} \prod_{\kappa=1}^{k} \prod_{s=1}^{N_{f}}\left[\frac{\varphi_{\kappa}-\mathrm{i} \tilde{m}_{s}}{\varphi_{\kappa}+1+\mathrm{i} m_{s}+n_{s} \mathrm{i} m_{X}} \frac{\varphi_{\kappa}+\mathrm{i} m_{s}-\mathrm{i} m_{X}}{\varphi_{\kappa}+\mathrm{i} m_{s}+\left(n_{s}-1\right) \mathrm{i} m_{X}}\right]\right\}
\end{aligned}
$$

whose residues include all contributions to the $k$-vortex partition function $Z_{\mathrm{v},\left\{n_{s}\right\}, k}$. As in the SQCD case, we move the pole at infinity to a finite value through a regulating factor, which depends on large real parameters with $1 \ll\left|M_{1}\right| \ll \cdots \ll\left|M_{k}\right|$. The small shift by $\frac{1}{2}$ moves poles away from the imaginary axis. We assume that the complex parameters $m_{s}$ and $m_{X}$ are in the ranges

$$
0<\operatorname{Re}\left(\mathrm{i} m_{X}\right)<1, \quad\left(n_{s}-1\right) \operatorname{Re}\left(\mathrm{i} m_{X}\right)<\operatorname{Re}\left(-\mathrm{i} m_{s}\right)<n_{s} \operatorname{Re}\left(\mathrm{i} m_{X}\right) .
$$

This constraint is eventually lifted since the relation we will deduce between vortex partition functions is analytic in $m_{s}$ and $m_{X}$. 
Close the contours of (B.42) towards $+\infty$ first. Because of the factors $1 /\left(\varphi_{\kappa}-\varphi_{\lambda}-1\right)$ and $1 /\left(\varphi_{\kappa}-\varphi_{\lambda}-\mathrm{i} m_{X}\right)$, the surrounded poles are such that the $\varphi_{\lambda}$ are organized into groups of components with related values:

$$
\begin{aligned}
& \left\{\varphi_{\lambda} \mid \lambda \in T\right\}=\coprod_{\kappa \in K}\left\{\frac{1}{2}+\epsilon_{\kappa} \mathrm{i} M_{\kappa}+\nu \mathrm{i} m_{X}+j \mid 0 \leq \nu<\hat{n}_{\kappa}, 0 \leq j<\hat{k}_{\kappa \nu}\right\}, \\
& \left\{\varphi_{\lambda} \mid \lambda \in S\right\}=\coprod_{1 \leq s \leq N_{f}}\left\{-\mathrm{i} m_{s}+\left(1-n_{s}+\mu\right) \mathrm{i} m_{X}+i \mid 0 \leq \mu<n_{s}^{\prime}, 0 \leq i<k_{s \mu}^{\prime}\right\}
\end{aligned}
$$

where $K$ is the set of indices for which $\varphi_{\kappa}=\frac{1}{2}+\epsilon_{\kappa} \mathrm{i} M_{\kappa}$, and $\llbracket 1, k \rrbracket=S \sqcup T$. Note that all $n_{s}^{\prime} \leq n_{s}$, otherwise the numerator factor $\prod_{\lambda}\left(\varphi_{\lambda}+\mathrm{i} m_{s}-\mathrm{i} m_{X}\right)$ would vanish. Introducing if necessary $k_{s n_{s}^{\prime}}^{\prime}=\cdots=k_{s\left(n_{s}-1\right)}^{\prime}=0$, we set $n_{s}^{\prime}=n_{s}$, then define $k_{s \mu}=k_{s\left(n_{s}-1-\mu\right)}^{\prime}$.

The pole is uniquely determined by the partition $\llbracket 1, k \rrbracket=S \sqcup T$, the set $K \subseteq T$, the signs $\epsilon_{\kappa}= \pm 1$, the non-negative integers $n_{s}$ (fixed when defining $I_{k}$ ), $k_{s \mu}, \hat{n}_{\kappa}$, and $\hat{k}_{\kappa \nu}$, and the maps $\sigma$ and $\tau$ defined by

$$
\varphi_{\sigma(s, \mu, i)}=-\mathrm{i} m_{s}-\mu \mathrm{i} m_{X}+i \quad \text { and } \quad \varphi_{\tau(\kappa, \nu, j)}=\frac{1}{2}+\epsilon_{\kappa} \mathrm{i} M_{\kappa}+\nu \mathrm{i} m_{X}+j
$$

for $1 \leq s \leq N_{f}, 0 \leq \mu<n_{s}, 0 \leq i<k_{s \mu}$, and for $\kappa \in K, 0 \leq \mu<\hat{n}_{\kappa}, 0 \leq j<\hat{k}_{\kappa \mu}$. This data is constrained: $\sigma$ is a bijection from $\left\{(s, \mu, i) \mid 0 \leq i<k_{s \mu}\right\}$ to $S$, hence $\sum k_{s \mu}=\# S$, and $\tau$ is a bijection from $\left\{(\kappa, \nu, j) \mid 0 \leq j<\hat{k}_{\kappa \nu}\right\}$ to $T$, hence $\sum \hat{k}_{\kappa \nu}=\# T$. Also, $\tau(\kappa, 0,0)=\kappa$ for all $\kappa \in K$.

Let $t=\# T$. It is convenient to parametrize poles in terms of the data $t, T$, $\left(K, \hat{n}_{\kappa}, \hat{k}_{\kappa \nu}, \tau\right),\left(k_{s \mu}, \sigma\right)$, and $\epsilon_{\kappa}$. When summing residues of $I_{k}$ at such poles, we will first sum over choices of signs $\epsilon_{\kappa}$ and take the limits $\left|M_{\kappa}\right| \rightarrow \infty$. The result is independent of $\sigma$, which thus contributes only a combinatorical factor. Then follows a sum over choices of $k_{s \mu}$, whose only constraint is $\sum k_{s \mu}=k-t$. Since the residue of $I_{k}$ involves the vortex contribution $V_{\left\{n_{s}\right\}}^{\left\{k_{s \mu}\right\}}$, the sum over $k_{s \mu}$ yields the $(k-t)$-vortex partition function. Summing over the remaining data, we find that $I_{k}$ is a linear combination of $(k-t)$-vortex partition functions for $0 \leq t \leq k$, whose coefficients only depend on $t$, i $m_{X}$, and a single combination $\Sigma$ of the twisted masses. This allows us to fix the coefficients by considering a simple case.

Let us proceed. The residue at (B.46) of (B.42) has the following asymptotics:

$$
\begin{aligned}
\prod_{\kappa=1}^{k}\left[1+O\left(\frac{1}{M_{\kappa}^{2}}\right)\right] \prod_{\kappa<\lambda}^{k}\left[1+O\left(\frac{M_{\kappa}^{2}}{M_{\lambda}^{2}}\right)\right] \prod_{\tau(\kappa, \mu, j)<\kappa}\left[O\left(\frac{M_{\tau(\kappa, \mu, j)}^{2}}{M_{\kappa}^{2}}\right)\right] \\
\cdot \frac{(-1)^{k}}{k !} \prod_{\kappa \in K} f_{\left\{\hat{n}_{\kappa}\right\},\left\{\hat{k}_{\kappa \nu}\right\}}\left(\mathrm{i} m_{X}\right) \prod_{\kappa \in K}\left[\frac{-\epsilon_{\kappa} \mathrm{i} M_{\kappa}}{2}+\frac{\Sigma}{2} \sum_{\nu=0}^{\hat{n}_{\kappa}-1} \hat{k}_{\kappa \nu}+O\left(\frac{1}{M_{\kappa}}\right)\right] V_{\left\{n_{s}\right\}}^{\left\{k_{s \mu}\right\}}
\end{aligned}
$$

where $f$ is a rational function of $\mathrm{i} m_{X}$ with integer coefficients, and

$$
\Sigma=2 N \mathrm{i} m_{X}+\sum_{s=1}^{N_{f}}\left(1+\mathrm{i} m_{s}+\mathrm{i} \widetilde{m}_{s}\right) .
$$


We expect the divergent piece $-\epsilon_{\kappa} \mathrm{i} M_{\kappa} / 2$ of the residue to cancel when summing over signs $\epsilon_{\kappa}$. Let us take limits $\left|M_{\lambda}\right| \rightarrow \infty$ from $\lambda=k$ down to $\lambda=1$ carefully. At each step there are two cases. If $\lambda \in K$, then the limit vanishes whenever any $\tau(\lambda, \mu, j)<\lambda$. Hence, only poles with all $\tau(\lambda, \mu, j) \geq \lambda$ contribute and we can focus on those. The $M_{\lambda}$-dependent terms are then of the form $-\epsilon_{\lambda} \mathrm{i} M_{\lambda} / 2$ plus a finite part. Summing over $\epsilon_{\lambda}= \pm 1$ only leaves the finite part. On the other hand, if $\lambda \notin K$, then taking the limit $\left|M_{\lambda}\right| \rightarrow \infty$ is trivial as $M_{\lambda}$ only appears in factors $\left[1+O\left(1 / M_{\lambda}^{2}\right)\right]$ and $\left[1+O\left(M_{\kappa}^{2} / M_{\lambda}^{2}\right)\right]$ for $\kappa<\lambda$ (importantly, we have already taken the limits $\left|M_{\kappa}\right| \rightarrow \infty$ for all $\left.\kappa>\lambda\right)$.

All in all, we are left with a non-divergent expression for $I$ :

$$
I_{k}=\frac{1}{k !} \sum_{t} \sum_{T} \sum_{K,\left\{\hat{n}_{\kappa}\right\},\left\{\hat{k}_{\kappa \nu}\right\}, \tau} \sum_{\left\{k_{s \mu}\right\}, \sigma} \Sigma^{\# K} \prod_{\kappa \in K}\left[f_{\left\{\hat{n}_{\kappa}\right\},\left\{\hat{k}_{\kappa \nu}\right\}}\left(\mathrm{i} m_{X}\right) \sum_{\nu=0}^{\hat{n}_{\kappa}-1} \hat{k}_{\kappa \nu}\right] V_{\left\{n_{s}\right\}}^{\left\{k_{s \mu}\right\}}
$$

The summand is independent of $\sigma$, and there are $(k-t)$ ! maps $\sigma$. Summing $V_{\left\{n_{s}\right\}}^{\left\{k_{s \mu}\right\}}$ over $k_{s \mu}$ with $\sum k_{s \mu}=k-t$ yields $Z_{\mathrm{v},\left\{n_{s}\right\}, k-t}$. The sum over $K, \hat{n}_{\kappa}, \hat{k}_{\kappa \nu}, \tau$ does not depend on the precise set $T$, but only on $t=\# T$. The choice of $T$ thus simply contributes a factor $k ! /[t !(k-t) !]$, which cancels the overall $1 / k$ !, and $(k-t)$ ! coming from the choice of $\sigma$. For a fixed $j=\# K$, the remaining sums yield a rational function of $\mathrm{i} m_{X}$ which can only depend on the two integers $0 \leq j \leq t \leq k$ :

$$
I_{k}=\sum_{t=0}^{k} \sum_{j=0}^{t} f_{t j}\left(\mathrm{i} m_{X}\right) \Sigma^{j} Z_{\mathrm{v},\left\{n_{s}\right\}, k-t} .
$$

Since the $f_{t j}$ do not depend on $k$, summing over $k$ yields

$$
\sum_{k \geq 0} y^{k} I_{k}=\sum_{t \geq 0} \sum_{j=0}^{t}\left[y^{t} f_{t j}\left(\mathrm{i} m_{X}\right) \Sigma^{j}\right] Z_{\mathrm{v},\left\{n_{s}\right\}}(y)=f\left(\mathrm{i} m_{X}, \Sigma ; y\right) Z_{\mathrm{v},\left\{n_{s}\right\}}(y) .
$$

In appendix B.2.1, we consider the case $N_{f}=1, n_{1}=N, 1+\mathrm{i} m_{1}+\mathrm{i} \widetilde{m}_{1}+N \mathrm{i} m_{X}=0$, for which $\Sigma=N i m_{X}$, and find that

$$
Z_{\mathrm{v},\{N\}}\left(1+\mathrm{i} m_{1}+\mathrm{i} \widetilde{m}_{1}+N \mathrm{i} m_{X}=0 ; y\right)=(1-y)^{-N\left(\mathrm{i} m_{X}+1\right)}=(1-y)^{-\left[1+1 /\left(\mathrm{i} m_{X}\right)\right] \Sigma} .
$$

On the other hand, since the factors $\varphi_{\kappa}-\mathrm{i} \widetilde{m}_{1}$ and $\varphi_{\kappa}+1+\mathrm{i} m_{1}+n_{1} \mathrm{i} m_{X}$ in (B.42) cancel, the integrand of $I_{k}$ has no pole with $\operatorname{Re}\left(\varphi_{\kappa}\right)<0$, thus $I_{k}=\delta_{k 0}$. As a result,

$$
f\left(\mathrm{i} m_{X}, \Sigma ; y\right)=(1-y)^{\left[1+1 /\left(\mathrm{i} m_{X}\right)\right] \Sigma}
$$

for all $\Sigma=N \mathrm{i} m_{X}$. This fixes each polynomial $\sum_{j=0}^{t} f_{t j}\left(\mathrm{i} m_{X}\right) \Sigma^{j}$ at an infinite set of values, hence determines $f$ completely.

At last, we are ready to wrap up, by showing that $I_{k}$ is the $k$-vortex partition function of the dual theory. Close contours of (B.42) towards $-\infty$. The surrounded poles are labeled by non-negative integers $n_{t}^{\prime} \geq 0$ and $k_{t \nu}^{\prime} \geq 0$ for $1 \leq t \leq N_{f}$ and $0 \leq \nu<n_{t}^{\prime}$ :

$$
\left\{\varphi_{\kappa}\right\}=\left\{-1-\mathrm{i} m_{t}-n_{t} \mathrm{i} m_{X}-\nu \mathrm{i} m_{X}-j \mid 0 \leq \nu<n_{t}^{\prime}, 0 \leq j<k_{t \nu}^{\prime}\right\} .
$$


For the choice of superpotential $W=\sum_{t=1}^{N_{f}} \widetilde{q}_{t} X^{l_{t}} q_{t}$, the constraint $1+\mathrm{i} m_{t}+\mathrm{i} \widetilde{m}_{t}+l_{t} \mathrm{i} m_{X}=0$ implies that the numerator factor $\prod_{\kappa}\left(\varphi_{\kappa}-\mathrm{i} \widetilde{m}_{t}\right)$ vanishes unless all $k_{t \nu}^{\prime}=0$ for $\nu \geq l_{t}-n_{t}$. For the choice of superpotential $W=\operatorname{Tr} X^{l+1}$, the constraint $1+(l+1) \mathrm{i} m_{X}=0$ implies that $\prod_{\kappa}\left(\varphi_{\kappa}+\mathrm{i} m_{t}-\mathrm{i} m_{X}\right)$ vanishes unless all $k_{t \nu}^{\prime}=0$ for $\nu \geq l-n_{t}$. We can thus take $n_{t}^{\prime}=l_{t}-n_{t}$ in both cases, and let $k_{t \nu}=k_{t\left(l_{t}-n_{t}-1-\nu\right)}^{\prime}$ so that

$$
\left\{\varphi_{\kappa}\right\}=\left\{-1-\mathrm{i} m_{t}-\left(l_{t}-1-\nu\right) \mathrm{i} m_{X}-j \mid 0 \leq \nu<l_{t}-n_{t}, 0 \leq j<k_{t \nu}\right\} .
$$

Summing over residues yields, after some massaging,

$$
\begin{gathered}
I_{k}=\sum_{\left\{k_{t \nu} \geq 0 \mid 0 \leq \nu<l_{t}-n_{t}\right\}} \prod_{(s, \mu)}\left[\prod_{(t, \nu)} \frac{\left(\mathrm{i} m_{s}-\mathrm{i} m_{t}+\left(l_{s}-l_{t}+\nu-\mu-1\right) \mathrm{i} m_{X}-k_{t \nu}\right)_{k_{s \mu}}}{\left(\mathrm{i} m_{s}-\mathrm{i} m_{t}+\left(l_{s}-l_{t}+\nu-\mu\right) \mathrm{i} m_{X}-k_{t \nu}\right)_{k_{s \mu}}}\right. \\
\left.\cdot \prod_{t=1}^{N_{f}}\left[\frac{\left(1+\mathrm{i} \widetilde{m}_{t}+\mathrm{i} m_{s}+\left(l_{s}-1-\mu\right) \mathrm{i} m_{X}\right)_{k_{s \mu}}}{\left(\mathrm{i} m_{s}-\mathrm{i} m_{t}+\left(l_{s}-l_{t}-1-\mu\right) \mathrm{i} m_{X}\right)_{k_{s \mu}}} \frac{\left.\left(\mathrm{i} m_{s}-\mathrm{i} m_{t}+\left(l_{s}-\mu\right) \mathrm{i} m_{X}+1\right)_{k_{s \mu}}-\mathrm{i} m_{t}+\left(l_{s}-n_{t}-\mu\right) \mathrm{i} m_{X}+1\right)_{k_{s \mu}}}{\left(\mathrm{i} m_{s}\right.}\right]\right] .
\end{gathered}
$$

For $W=\sum_{t=1}^{N_{f}} \widetilde{q}_{t} X^{l_{t}} q_{t}$, the summand takes the general form (B.40) of $V_{\left\{l_{t}-n_{t}\right\}}^{\{k\}}$, with $m_{t} \leftrightarrow \widetilde{m}_{t}$ since $1+\mathrm{i} \widetilde{m}_{t}+\mathrm{i} m_{s}+\left(l_{s}-1-\mu\right) \mathrm{i} m_{X}=\mathrm{i} m_{s}-\mathrm{i} m_{t}+\left(l_{s}-l_{t}-1-\mu\right) \mathrm{i} m_{X}$. Thus, $I_{k}$ is the $k$-vortex partition function of the SQCDA theory with $N_{f}$ flavour, $L-N$ colors, the superpotential $W=\sum_{t} \widetilde{q}_{t} X^{l_{t}} q_{t}$, interchanged twisted masses $m_{t} \leftrightarrow \widetilde{m}_{t}$ compared to the $\mathrm{U}(N)$ theory, and the same value of $y$. Charge conjugation maps twisted masses back to those of the $\mathrm{U}(N)$ theory, and maps $y \rightarrow y^{D}=y^{-1}$ hence $z^{D}=z^{-1}$. Summing $y^{k} I_{k}$ then yields the $\mathrm{u}$-channel vortex partition function of the $\mathrm{U}(L-N)$ theory (that is, a series in powers of $\left.\left(y^{D}\right)^{-1}\right)$. We finally combine the relation (B.51) and the explicit factor (B.53) with $\Sigma=(2 N-L)$ i $m_{X}$ to get

$$
Z_{\mathrm{v},\left\{l_{s}-n_{s}\right\}}^{\mathrm{U}(L-N)}\left(\left(y^{D}\right)^{-1}\right)=(1-y)^{(2 N-L)\left(1+\mathrm{i} m_{X}\right)} Z_{\mathrm{v},\left\{n_{s}\right\}}^{\mathrm{U}(N)}(y) \quad \text { for } W=\sum_{t=1}^{N_{f}} \widetilde{q}_{t} X^{l_{t}} q_{t} .
$$

For $W=\operatorname{Tr} X^{l+1}$, we have $\mathrm{i} m_{s}-\mathrm{i} m_{t}+\left(l_{s}-l_{t}-1-\mu\right) \mathrm{i} m_{X}=\mathrm{i} m_{s}-\mathrm{i} m_{t}+\left(l_{s}-\mu\right) \mathrm{i} m_{X}+1$, and again the summand takes the form of $V_{\left\{l_{t}-n_{t}\right\}}^{\{k\}}$, with $\mathrm{i} m_{s} \rightarrow \mathrm{i} m_{s}^{D}=\mathrm{i} m_{X}-\mathrm{i} m_{s}$ and $\mathrm{i} \widetilde{m}_{t} \rightarrow \mathrm{i} \widetilde{m}_{t}^{D}=\mathrm{i} m_{X}-\mathrm{i} \widetilde{m}_{t}$. Combining the relation (B.51) and the explicit factor (B.53) with $\Sigma=2 N \mathrm{i} m_{X}+\sum_{t=1}^{N_{f}}\left(1+\mathrm{i} m_{t}+\mathrm{i} \widetilde{m}_{t}\right)$ and $1+1 /\left(\mathrm{i} m_{X}\right)=-l$ yields the Kutasov-Schwimmer duality relation

$$
Z_{\mathrm{v},\left\{l-n_{s}\right\}}^{\mathrm{U}\left(l N_{f}-N\right)}\left(m_{t}^{D}, \widetilde{m}_{t}^{D} ; y^{D}\right)=(1-y)^{-\delta_{1}} Z_{\mathrm{v},\left\{n_{s}\right\}}^{\mathrm{U}(N)}\left(m_{t}, \widetilde{m}_{t} ; y\right) \quad \text { for } W=\operatorname{Tr} X^{l+1}
$$

with $\delta_{1}=-\frac{2 l}{l+1} N+l \sum_{t=1}^{N_{f}}\left(1+\mathrm{i} m_{t}+\mathrm{i} \widetilde{m}_{t}\right)$, as obtained in (3.42) through the relation with conjugation of momenta in the Toda CFT. Since $y^{D}=y$, we get $z^{D}=z$. In section 3.2.2, we extend the Kutasov-Schwimmer duality to theories with a different number of fundamental and antifundamental chiral multiplets.

Open Access. This article is distributed under the terms of the Creative Commons Attribution License (CC-BY 4.0), which permits any use, distribution and reproduction in any medium, provided the original author(s) and source are credited. 


\section{References}

[1] K.G. Wilson, Confinement of quarks, Phys. Rev. D 10 (1974) 2445 [inSPIRE].

[2] G. 't Hooft, On the phase transition towards permanent quark confinement, Nucl. Phys. B 138 (1978) 1 [INSPIRE].

[3] S. Gukov and E. Witten, Gauge theory, ramification, and the geometric Langlands program, hep-th/0612073 [INSPIRE].

[4] S. Gukov and A. Kapustin, Topological quantum field theory, nonlocal operators and gapped phases of gauge theories, arXiv:1307.4793 [INSPIRE].

[5] J. Gomis and S. Matsuura, Bubbling surface operators and S-duality, JHEP 06 (2007) 025 [arXiv: 0704.1657] [INSPIRE].

[6] S. Gukov, Gauge theory and knot homologies, Fortsch. Phys. 55 (2007) 473 [arXiv:0706.2369] [INSPIRE].

[7] E. Witten, Gauge theory and wild ramification, arXiv:0710.0631 [INSPIRE].

[8] E.I. Buchbinder, J. Gomis and F. Passerini, Holographic gauge theories in background fields and surface operators, JHEP 12 (2007) 101 [arXiv:0710.5170] [INSPIRE].

[9] C. Beasley, J.J. Heckman and C. Vafa, GUTs and exceptional branes in F-theory - I, JHEP 01 (2009) 058 [arXiv:0802 .3391] [INSPIRE].

[10] S. Gukov and E. Witten, Rigid surface operators, Adv. Theor. Math. Phys. 14 (2010) 87 [arXiv: 0804.1561] [INSPIRE].

[11] N. Drukker, J. Gomis and S. Matsuura, Probing $N=4$ SYM with surface operators, JHEP 10 (2008) 048 [arXiv: 0805.4199] [INSPIRE].

[12] D. Gaiotto, Surface operators in $N=24 D$ gauge theories, JHEP 11 (2012) 090 [arXiv:0911.1316] [INSPIRE].

[13] D. Gaiotto, $N=2$ dualities, JHEP 08 (2012) 034 [arXiv:0904.2715] [INSPIRE].

[14] V. Pestun, Localization of gauge theory on a four-sphere and supersymmetric Wilson loops, Commun. Math. Phys. 313 (2012) 71 [arXiv:0712.2824] [InSPIRE].

[15] N. Hama and K. Hosomichi, Seiberg-Witten theories on ellipsoids, JHEP 09 (2012) 033 [arXiv:1206.6359] [INSPIRE].

[16] J. Gomis, T. Okuda and V. Pestun, Exact results for 't Hooft loops in gauge theories on $S^{4}$, JHEP 05 (2012) 141 [arXiv: 1105.2568] [INSPIRE].

[17] F. Benini and S. Cremonesi, Partition functions of $\mathcal{N}=(2,2)$ gauge theories on $S^{2}$ and vortices, Commun. Math. Phys. 334 (2015) 1483 [arXiv:1206.2356] [InSPIRE].

[18] N. Doroud, J. Gomis, B. Le Floch and S. Lee, Exact results in $D=2$ supersymmetric gauge theories, JHEP 05 (2013) 093 [arXiv:1206.2606] [INSPIRE].

[19] J. Gomis and S. Lee, Exact Kähler potential from gauge theory and mirror symmetry, JHEP 04 (2013) 019 [arXiv:1210.6022] [INSPIRE].

[20] N. Doroud and J. Gomis, Gauge theory dynamics and Kähler potential for Calabi-Yau complex moduli, JHEP 12 (2013) 99 [arXiv:1309.2305] [INSPIRE].

[21] L.F. Alday, D. Gaiotto and Y. Tachikawa, Liouville correlation functions from four-dimensional gauge theories, Lett. Math. Phys. 91 (2010) 167 [arXiv:0906.3219] [INSPIRE]. 
[22] N. Wyllard, $A_{N-1}$ conformal Toda field theory correlation functions from conformal $N=2$ $\mathrm{SU}(N)$ quiver gauge theories, JHEP 11 (2009) 002 [arXiv:0907.2189] [INSPIRE].

[23] L.F. Alday, D. Gaiotto, S. Gukov, Y. Tachikawa and H. Verlinde, Loop and surface operators in $N=2$ gauge theory and Liouville modular geometry, JHEP 01 (2010) 113 [arXiv:0909.0945] [INSPIRE].

[24] N. Drukker, J. Gomis, T. Okuda and J. Teschner, Gauge theory loop operators and Liouville theory, JHEP 02 (2010) 057 [arXiv: 0909.1105] [INSPIRE].

[25] N. Drukker, D. Gaiotto and J. Gomis, The virtue of defects in $4 D$ gauge theories and $2 D$ CFTs, JHEP 06 (2011) 025 [arXiv: 1003.1112] [INSPIRE].

[26] F. Passerini, Gauge theory Wilson loops and conformal Toda field theory, JHEP 03 (2010) 125 [arXiv: 1003.1151] [INSPIRE].

[27] J. Gomis and B. Le Floch, 't Hooft operators in gauge theory from Toda CFT, JHEP 11 (2011) 114 [arXiv:1008.4139] [INSPIRE].

[28] M. Bullimore, Defect networks and supersymmetric loop operators, JHEP 02 (2015) 066 [arXiv:1312.5001] [INSPIRE].

[29] T. Dimofte, S. Gukov and L. Hollands, Vortex counting and lagrangian 3-manifolds, Lett. Math. Phys. 98 (2011) 225 [arXiv:1006.0977] [INSPIRE].

[30] M. Taki, Surface Operator, Bubbling Calabi-Yau and AGT Relation, JHEP 07 (2011) 047 [arXiv: 1007.2524] [INSPIRE].

[31] G. Bonelli, A. Tanzini and J. Zhao, Vertices, vortices and interacting surface operators, JHEP 06 (2012) 178 [arXiv:1102.0184] [INSPIRE].

[32] G. Bonelli, A. Tanzini and J. Zhao, The Liouville side of the vortex, JHEP 09 (2011) 096 [arXiv: 1107.2787] [INSPIRE].

[33] A. Braverman, B. Feigin, M. Finkelberg and L. Rybnikov, A finite analog of the AGT relation I: finite W-algebras and quasimaps' spaces, Commun. Math. Phys. 308 (2011) 457 [arXiv: 1008.3655] [INSPIRE].

[34] L.F. Alday and Y. Tachikawa, Affine SL(2) conformal blocks from $4 D$ gauge theories, Lett. Math. Phys. 94 (2010) 87 [arXiv:1005.4469] [InSPIRE].

[35] C. Kozcaz, S. Pasquetti, F. Passerini and N. Wyllard, Affine sl $(N)$ conformal blocks from $N=2 \mathrm{SU}(N)$ gauge theories, JHEP 01 (2011) 045 [arXiv: 1008.1412] [INSPIRE].

[36] N. Wyllard, $W$-algebras and surface operators in $N=2$ gauge theories, J. Phys. A 44 (2011) 155401 [arXiv:1011.0289] [InSPIRE].

[37] N. Wyllard, Instanton partition functions in $N=2 \mathrm{SU}(N)$ gauge theories with a general surface operator and their W-algebra duals, JHEP 02 (2011) 114 [arXiv:1012.1355] [INSPIRE].

[38] Y. Tachikawa, On $W$-algebras and the symmetries of defects of $6 d N=(2,0)$ theory, JHEP 03 (2011) 043 [arXiv:1102.0076] [INSPIRE].

[39] H. Kanno and Y. Tachikawa, Instanton counting with a surface operator and the chain-saw quiver, JHEP 06 (2011) 119 [arXiv:1105.0357] [INSPIRE].

[40] V. Belavin, Conformal blocks of chiral fields in $N=2$ SUSY CFT and affine Laumon spaces, JHEP 10 (2012) 156 [arXiv:1209.2992] [INSPIRE]. 
[41] M.-C. Tan, M-theoretic derivations of 4D-2D dualities: from a geometric Langlands duality for surfaces, to the AGT correspondence, to integrable systems, JHEP 07 (2013) 171 [arXiv: 1301.1977] [INSPIRE].

[42] A. Gadde and S. Gukov, 2D index and surface operators, JHEP 03 (2014) 080 [arXiv: 1305. 0266] [INSPIRE].

[43] Y. Nakayama, $4 D$ and $2 D$ superconformal index with surface operator, JHEP 08 (2011) 084 [arXiv: 1105.4883] [INSPIRE].

[44] D. Gaiotto, L. Rastelli and S.S. Razamat, Bootstrapping the superconformal index with surface defects, JHEP 01 (2013) 022 [arXiv:1207.3577] [INSPIRE].

[45] L.F. Alday, M. Bullimore, M. Fluder and L. Hollands, Surface defects, the superconformal index and q-deformed Yang-Mills, JHEP 10 (2013) 018 [arXiv:1303.4460] [INSPIRE].

[46] M. Bullimore, M. Fluder, L. Hollands and P. Richmond, The superconformal index and an elliptic algebra of surface defects, JHEP 10 (2014) 62 [arXiv:1401.3379] [INSPIRE].

[47] E. Witten, Phases of $N=2$ theories in two-dimensions, Nucl. Phys. B 403 (1993) 159 [hep-th/9301042] [INSPIRE].

[48] F. Benini, D.S. Park and P. Zhao, Cluster algebras from dualities of $2 D \mathcal{N}=(2,2)$ quiver gauge theories, Commun. Math. Phys. 340 (2015) 47 [arXiv:1406.2699] [InSPIRE].

[49] K. Hori and D. Tong, Aspects of non-abelian gauge dynamics in two-dimensional $N=(2,2)$ theories, JHEP 05 (2007) 079 [hep-th/0609032] [INSPIRE].

[50] N. Seiberg, Electric-magnetic duality in supersymmetric nonAbelian gauge theories, Nucl. Phys. B 435 (1995) 129 [hep-th/9411149] [InSPIRE].

[51] D. Gaiotto and J. Teschner, Irregular singularities in Liouville theory and Argyres-Douglas type gauge theories, I, JHEP 12 (2012) 050 [arXiv: 1203.1052] [INSPIRE].

[52] D. Kutasov, A comment on duality in $N=1$ supersymmetric non-Abelian gauge theories, Phys. Lett. B 351 (1995) 230 [hep-th/9503086] [INSPIRE].

[53] D. Kutasov and A. Schwimmer, On duality in supersymmetric Yang-Mills theory, Phys. Lett. B 354 (1995) 315 [hep-th/9505004] [INSPIRE].

[54] K. Hori and M. Romo, Exact results in two-dimensional $(2,2)$ supersymmetric gauge theories with boundary, arXiv:1308.2438 [INSPIRE].

[55] D. Honda and T. Okuda, Exact results for boundaries and domain walls in $2 d$ supersymmetric theories, JHEP 09 (2015) 140 [arXiv:1308.2217] [INSPIRE].

[56] C. Closset and S. Cremonesi, Comments on $\mathcal{N}=(2,2)$ supersymmetry on two-manifolds, JHEP 07 (2014) 075 [arXiv: 1404.2636] [INSPIRE].

[57] E. Gerchkovitz, J. Gomis and Z. Komargodski, Sphere partition functions and the Zamolodchikov metric, JHEP 11 (2014) 001 [arXiv: 1405.7271] [INSPIRE].

[58] V.A. Fateev and A.V. Litvinov, Correlation functions in conformal Toda field theory. I., JHEP 11 (2007) 002 [arXiv:0709.3806] [INSPIRE].

[59] D. Orlando and S. Reffert, Relating gauge theories via gauge/bethe correspondence, JHEP 10 (2010) 071 [arXiv:1005.4445] [INSPIRE].

[60] H. Kanno, K. Maruyoshi, S. Shiba and M. Taki, $W_{3}$ irregular states and isolated $N=2$ superconformal field theories, JHEP 03 (2013) 147 [arXiv:1301.0721] [INSPIRE].

[61] D. Gaiotto and J. Lamy-Poirier, Irregular singularities in the $H_{3}^{+} \mathrm{WZW}$ model, arXiv:1301.5342 [INSPIRE]. 